\title{
Caracterização por espalhamento de luz de dispersões aquosas de agregados lipídicos aniônicos
}

\author{
Thais Azevedo Enoki
}

Orientadora: Profa. Dra. Maria Teresa Moura Lamy

BANCA EXAMINADORA

$\operatorname{Prof}^{a}$. Dr ${ }^{a}$. Maria Teresa Moura Lamy (IFUSP)

Prof. Dr. Leandro Ramos Souza Barbosa (IFUSP)

$\operatorname{Prof}^{a}$. Dr ${ }^{a}$. Nadya Pesce Silveira (UFRGS)

São Paulo, SP 



\section{Agradecimentos}

Agradeço primeiramente a Deus.

Gostaria de agradecer à $\operatorname{Prof}^{a}$ Maria Teresa Lamy, pela oportunidade, dedicação e preocupação com o desenvolvimento deste projeto, e pelo cuidado dado à minha formação. Também gostaria de agradecer à Prof ${ }^{a}$ Vera B. Henriques, que sempre participou das discussões deste trabalho e incentivou o desenvolvimento dos estudos teóricos.

Agradeço à Prof ${ }^{a}$ Carla Goldman, pelas discussões sobre espalhamento de luz dinâmico.

Agradeço ao apoio financeiro do CNPq.

Agradeço a todo o grupo de Biofísica, em especial aos experimentais, pela ótima convivência no laboratório, pelas discussões, sugestões e auxílios diversos. Mais especificamente, agradeço ao Tiago, pela ajuda inicial com os equipamentos e pelas discussões teóricas de espalhamento de lus estático; ao Evandro, pela paciência e habilidade para alinhar o laser sempre que necessário, e pelos conselhos; ao Rafael pelo compartilhamento de informações sobre o DMPG não extrusado; à Daniela, pela ajuda e pela força; e à Cintia pelos conselhos.

Além dos agradecimentos diretamente relacionados a esse trabalho, gostaria de agradecer também às pessoas importantes na minha vida, que contribuíram, mesmo que indiretamente, para o bom desenvolvimento desse mestrado. Em especial, gostaria de agradecer aos meus pais, heróis de minha vida, Maria José Azevedo Enoki e Tosio Enoki, que nunca pouparam esforços para me ajudar e sempre me incentivaram muito; às minhas irmãs, Priscilla e Natacha, e ao meu novo sobrinho, Lucas, que sempre me contagiaram de alegria, e ao meu noivo, Danilo, pela imensa força, apoio, paciência, incentivo e companhia. Agradeço também aos amigos e colegas de trabalho do Instituto de Física da Universidade de São Paulo. 



\section{Resumo}

Neste trabalho caracterizamos dispersões aquosas de vesículas lipídicas por espalhamento de luz. Utilizamos os diferentes métodos da técnica: espalhamento de luz estático (SLS) e espalhamento de luz dinâmico (DLS) no estudo desses sistemas. No SLS a intensidade da luz espalhada foi coletada em vários ângulos de espalhamento para diferentes concentrações, e obtivemos os seguintes parâmetros: peso molecular, $M_{w}$, raio de giração, $R_{g}$, que esta relacionado à forma do centro espalhador e segundo coeficiente do virial, $A_{2}$, que está relacionado a interações entre partículas. No DLS a correlação da luz espalhada foi obtida em função do tempo, para vários ângulos e concentrações. As medidas de DLS fornecem o coeficiente de difusão dos centros espalhadores, que estão relacionados a seus diâmetros efetivos. Em especial a técnica de SLS foi amplamente estudada para desenvolvimento de uma apostila coordenada pela Prof ${ }^{a}$. Vera B. Henriques. Nesta apostila a teoria de espalhamento de luz estático está apresentada através de uma abordagem diferente da convencional, sob o ponto de vista da mecânica estatística. O principal objeto de estudo neste trabalho foram as dispersões aquosas de dimiristoil fosfatidil glicerol (DMPG). O DMPG, lipídio aniônico, saturado, com 14 carbonos nas cadeias hidrocarbônicas, em baixa força iônica, exibe um comportamento termo-estrutural muito peculiar, apresentando uma larga região de transição de fase, entre as fases gel e fluida. O lipídio dimiristoil fosfatidil colina (DMPC) com as mesmas cadeias carbônicas mas cabeça polar neutra, foi utilizado como controle, pois não apresenta esta anomalia. Os tamanhos efetivos obtidos pelos diferentes métodos (SLS) e (DLS) são semelhantes nas fases gel e fluída de ambos os lipídios, mas isso não ocorre na região de transição do DMPG. Ambos os métodos indicam um aumento na dimensão das vesículas de DMPG, nesta região de temperatura. No entanto, por SLS temos que o $R_{g}$ triplica na região de transição, levando a um raio efetivo muito maior que o observado por DLS. Como é sabido que não há fusão entre as vesículas, considerando os dados de espalhamento de luz e a literatura, discutimos um modelo em que o agregado lipídico apresente grandes deformações isotrópicas e grandes flutuações de forma. 



\section{Abstract}

Light scattering used to structurally characterize lipid dispersions. In the present work light scattering was used to characterize aqueous dispersions of lipid vesicles. Two different methods were applied: static light scattering (SLS) and dynamic light scattering (DLS). With SLS, the intensity of the scattered light was measured at several scattering angles and different lipid concentrations. The following parameters were obtained: molecular weight $\left(\bar{M}_{w}\right)$ and radius of gyration $\left(R_{g}\right)$, related to the mass and form of the scattering particle, and the second Virial coefficient $\left(A_{2}\right)$, related to interactions among particles. With DLS, the autocorrelation of the intensity of the scattered light was obtained, also at several scattering angles and lipid concentrations. Measurements of DLS provide the diffusion coefficient of the particles, which are related to their hydrodynamic radii. In particular, the theory of SLS was largely studied to the elaboration of a manuscript, under the supervision of Dr. Vera B. Henriques, which presents an unconventional approach to the technique. Here, the main object of investigation was the aqueous dispersion of the anionic lipid dimyristoyl phosphatidylglycerol (DMPG), a saturated anionic lipid, with 14 C-atoms in the hydrophobic chains. At low ionic strength, this lipid exhibits a very peculiar thermo-structural behavior, presenting a large region of transition, between the gel and fluid phases. The lipid dimyristoyl phosphatidylcholine (DMPC), with the same hydrocarbon chains but zwitterionic polar headgroup, was used as a control, since it does not present such anomaly. Lipid dispersions were extruded through $100 \mathrm{~nm}$ filters (pore diameter). In the gel and fluid phases of DMPC and DMPG, both SLS and DLS indicate similar dimensions for the lipid vesicles, assuming them spherical. For DMPC, the obtained effective radius $\left(R_{e f f}\right)$ values were around 53 and $62 \mathrm{~nm}$ for the gel and fluid phases, respectively, indicating the expected expansion of the fluid bilayer, and radii compatible with the extrusion process. For DMPG, the fluid vesicle $\left(R_{e f f} \approx 30 \mathrm{~nm}\right)$ was also found to be larger than the gel vesicle $\left(R_{e f f} \approx 27 \mathrm{~nm}\right)$, but much smaller than the dimensions of the filter pore $(R=50 \mathrm{~nm})$. More interesting, over the DMPG transition 
region, SLS indicated a three times increase for the vesicle radius of gyration, whereas the hydrodynamic radius, measured by DLS, increased by $30 \%$ only. Besides, no light depolarization could be detected, indicating that the DMPG aggregates are isotropic particles, in average, at all temperatures. Considering the results presented here, and those in the literature, the presence of large bilayer fluctuations over the DMPG transition region will be discussed, possibly including the existence of bilayer pores. 


\section{Sumário}

1 Introdução 1

2 Espalhamento de luz estático: Uma abordagem diferenciada $\quad 9$

2.1 Introdução . . . . . . . . . . . . . . . . . . . . . . . . 9

2.2 Espalhamento elástico de uma carga pontual . . . . . . . . . . . . . . 10

2.3 Interferência das ondas espalhadas por vários elétrons . . . . . . . . . . . . 12

2.4 Contribuição eletrônica de diferentes átomos . . . . . . . . . . . . . . . . . 14

2.5 Fator de Forma . . . . . . . . . . . . . . . . . . . . . . . . . 15

2.5.1 Fator de forma para modelos esféricos . . . . . . . . . . . 16

2.6 Fator de Estrutura . . . . . . . . . . . . . . . . . . . . . . . . . 18

2.7 Polarizabilidade . . . . . . . . . . . . . . . . . . . . . . . . . . . . . 19

2.8 Misturas . . . . . . . . . . . . . . . . . . . . . . . . . 22

2.9 Soluções . . . . . . . . . . . . . . . . . . . . . . . . . . . . . . 25

2.10 Relações termodinâmicas . . . . . . . . . . . . . . . . . . . . . 28

2.11 Expansão do fator de estrutura . . . . . . . . . . . . . . . . . 30

2.12 Expansão do fator de forma . . . . . . . . . . . . . . . . . . . . 31

2.13 Relação de Claussius Mossotti . . . . . . . . . . . . . . . . . . . . . . 32

2.14 A Equação de Zimm . . . . . . . . . . . . . . . . . . . . . . . . 34

$\begin{array}{lll}3 & \text { Espalhamento de luz dinâmico } & 37\end{array}$

3.1 Introdução . . . . . . . . . . . . . . . . . . . . . . . . . . 37

3.2 Função de Correlação . . . . . . . . . . . . . . . . . . . . . . . . . . 37

3.3 Correlator . . . . . . . . . . . . . . . . . . 38

3.4 Análises na função de correlação do campo elétrico . . . . . . . . . . . . 40

4 Materiais e métodos 43 
5 Técnicas experimentais complementares $\quad 49$

5.1 Absorção óptica . . . . . . . . . . . . . . . . . . . . . 49

5.1 .1 Lei de Lambert-Beer . . . . . . . . . . . . . . . . . . 49

5.1.2 Dosagem de fosfato: Determinação da concentração de fosfolipídios. 51

5.2 Refratômetro Diferencial . . . . . . . . . . . . . . . . 53

5.2.1 Incremento no índice de refração do DMPG . . . . . . . . . . . 56

5.2 .2 Incremento no índice de refração do DMPC . . . . . . . . . . . 58

5.3 Calorimetria Diferencial de Varredura . . . . . . . . . . . . . . . 61

6 Resultados e discussões $\quad 65$

6.1 Calorimetria Diferencial de Varredura . . . . . . . . . . . . . . . 65

6.2 Espalhamento de Luz Estático . . . . . . . . . . . . . . . . . . 69

6.3 Espalhamento de Luz Dinâmico . . . . . . . . . . . . . . . . . . . . 79

6.4 Comparação dos resultados, SLS e DLS . . . . . . . . . . . . . . . 93

6.5 Medidas de anisotropia do centro espalhador . . . . . . . . . . . . . 96

6.6 Discussões Gerais . . . . . . . . . . . . . . . . . . . . 98

$\begin{array}{llr}7 & \text { Conclusões } & 107\end{array}$

$\begin{array}{ll}\text { A Expansão dos Cumulantes } & 109\end{array}$

B Análise da função de auto correlação utilizando o programa Gendist 113

C Comparação do DMPG não extrusado e extrusado por DLS 119

$\begin{array}{ll}\text { Referências Bibliográficas } & 123\end{array}$ 


\section{Lista de Figuras}

1.1 Transição ordem-desordem dos lipídios. . . . . . . . . . . . . . . . . . . . . 1

1.2 Transição de fase altamente cooperativa do lipídio DMPC . . . . . . . . . . . . . . . . . 2

1.3 Comportamento termo-estrutural do DMPG a alta força iônica, $[\mathrm{NaCl}]=500 \mathrm{mM}$, gráfico cedido pelo aluno de doutorado Barroso, R.P. . . . . . . . . . . . . . . . . . . . 3

1.4 Comportamento termo-estrutural do DMPG $(1,0 \mathrm{mM})$ em baixa força iônica. . . . . . . . . 4

1.5 Aumento da região de transição para cadeias carbônicas curtas. . . . . . . . . . . . . . . . 6

1.6 Aumento da viscosidade relativa relacionada com a capacidade térmica e mudança estrutural na região de transição do DMPG. . . . . . . . . . . . . . . . . . . . . . . . . . . 6

2.1 Ilustração do modelo: elétron ligado oscilando com frequência natural $\omega_{0} . \quad$. . . . . . . . . 10

2.2 Intensidade da luz espalhada em função da frequência de oscilação $w$ e da frequência natural do elétron $\omega_{0} . \quad \ldots \ldots \ldots$. . . . . . . . . . . . . . . . . . . . . . . . . 11

2.3 Interferência da luz espalhada por duas partículas. . . . . . . . . . . . . . . . . . . . 12

2.4 A posição da partícula, em termos da posição do núcleo e da posição dos elétrons. . . . . . 14

2.5 Fator de forma da esfera, $\lambda=633$ nm. . . . . . . . . . . . . . . . . . . . 16

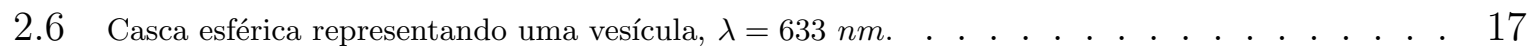

2.7 Fator de forma da casca esférica (vesícula), $\lambda=633 \mathrm{~nm} . \ldots \ldots \ldots$

2.8 Comparação dos fatores de forma da esfera e da casca esférica, $\lambda=633 \mathrm{~nm} . \quad$. . . . . . . 18

2.9 Fator de estrutura obtido a partir da transformada de Fourier da $g(r)$ dada pela aproximação de PY, para diferentes frações de volume ocupado. . . . . . . . . . . . . . . . . . . . . 20

2.10 Ilustração da polarizabilidade e do campo interno molecular. . . . . . . . . . . . . . . . 21

3.1 Flutuações da intensidade da luz espalhada no tempo, causada pelo movimento das moléculas no fluído. . . . . . . . . . . . . . . . . . . . . . . . . . . . . . . . . . 39

3.2 Trem de pulsos processados por um correlator. . . . . . . . . . . . . . . . . . . . . . 39

4.1 DMPG . . . . . . . . . . . . . . . . . . . . . 43

4.2 DMPC . . . . . . . . . . . . . . . . . . . . . . . 43 
5.1 Esquema ilustrativo do modelo proposto para deduzir a Lei de Lambert-Beer. . . . . . . . . 50

5.2 Dosagem de fosfato do DMPG, conjunto de amostras 1. . . . . . . . . . . . . . . . . . . 51

5.3 Dosagem de fosfato do DMPG, conjunto de amostras 2. . . . . . . . . . . . . . . . . . . 51

5.4 Dosagem de fosfato do DMPC, conjunto de amostras 1. . . . . . . . . . . . . . . . . . . 52

5.5 Dosagem de fosfato do DMPC, conjunto de amostras 2. . . . . . . . . . . . . . . . . . . 52

5.6 Esquema ilustrativo do refratômetro diferencial. . . . . . . . . . . . . . . . . . . . . 53

5.7 Curva de calibração: Ganho na voltagem com relação à concentração, para o $K C l$ a $43^{\circ} C . \quad$ • . $\quad 54$

5.8 Curva de calibração: Incremento do índice de refração com relação à concentração, para o $K C l$ а $43^{\circ}$ C. . . . . . . . . . . . . . . . . . . . . . . . . . . . . . . . . . . . . . . 54

5.9 Ganho na voltagem com relação à concentração, para o DMPG a $43^{\circ} \mathrm{C} . \quad$. . . . . . . . . . 55

5.10 Incremento do índice de refração com relação à concentração, para o DMPG a $43^{\circ} C$. . . . . 55

5.11 Curva de calibração: Ganho na voltagem com relação à concentração, para o $K C l$ a $20^{\circ} C . \quad$ • . 55

5.12 Curva de calibração: Incremento do índice de refração com relação à concentração, para o $\mathrm{KCl}$ a $20^{\circ}$ C. . . . . . . . . . . . . . . . . . . . . . . . . . . . . . . . . . 55

5.13 Curva de calibração: Ganho na voltagem com relação à concentração, para o $K C l$ a $28^{\circ} C . \quad$ • . 56

5.14 Curva de calibração: Incremento do índice de refração com relação à concentração, para o $K C l$ a $28^{\circ}$ C. . . . . . . . . . . . . . . . . . . . . . . . . . . 56

5.15 Ganho na voltagem com relação à concentração, para o DMPG a $20^{\circ}$ C . . . . . . . . . . . 56

5.16 Incremento do índice de refração com relação à concentração, para o DMPG a $20^{\circ} C$. . 56

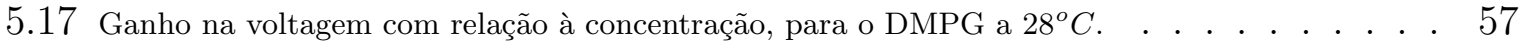

5.18 Incremento do índice de refração com relação à concentração, para o DMPG a $28^{\circ} C$. . . 57

5.19 Incremento do índice de refração, para o DMPG em $T=16^{\circ} C$, estimado pela regressão linear dos dados experimentais. . . . . . . . . . . . . . . . . . . . . . . . . . . . . 58

5.20 Ganho na voltagem com relação à concentração, para o DMPC a $20^{\circ}$ C . . . . . . . . . . 58

5.21 Incremento do índice de refração com relação à concentração, para o $\mathrm{DMPC}$ a $20^{\circ} C . \quad$. . . 58

5.22 Ganho na voltagem com relação à concentração, para o DMPC a $43^{\circ}$ C . . . . . . . . . . . . 59

5.23 Incremento do índice de refração com relação à concentração, para o $\mathrm{DMPC}$ a $43^{\circ} C . \quad$. . . . . 59

5.24 Incremento do índice de refração, para o DMPC em $T=23^{\circ} C$, estimado pela regressão linear dos dados experimentais. . . . . . . . . . . . . . . . . . . . . . . . . . . . . . . 59

5.25 Ilustração da medida de calorimetria. . . . . . . . . . . . . . . . . . . . . . . . . . . . 62 
6.1 Capacidade térmica em função da temperatura para o DMPG extrusado. As medidas de calorimetria foram realizadas com scan rate $20^{\circ} / \mathrm{h}$. As linhas pontilhadas ilustram as temperaturas em que realizamos as medidas de espalhamento de luz, SLS e DLS. As temperaturas são: $T=16^{\circ} C$ (fase gel), $T=20$ e $28^{\circ} C$ (região de transição) e $T=43^{\circ} C$ (fase fluida). As curvas de DSC foram deslocadas na vertical para facilitar a visualização. . . . . . . . . . . . . . .

6.2 Capacidade térmica em função da temperatura para o DMPG extrusado e não extrusado. As medidas de calorimetria foram realizadas com scan rate $20^{\circ} / h$. O gráfico ilustra três amostras distintas que foram preparadas nas mesmas condições. As curvas de DSC foram deslocadas na vertical para facilitar a visualização.

6.3 Capacidade térmica em função da temperatura para o DMPC extrusado e não extrusado. As medidas de calorimetria foram realizadas com scan rate $20^{\circ} / \mathrm{h}$. As linhas pontilhadas do gráfico da esquerda ilustram as temperaturas em que realizamos as medidas de espalhamento do luz, SLD e DLS. As temperaturas são: $T=20^{\circ} C$ (fase gel), $T=23$ (transição de fase ) e $T=43^{\circ} C$ (fase fluida). As curvas de DSC foram deslocadas na horizontal para facilitar a visualização. .

6.4 Gráficos de Zimm para o DMPG $(C=0,214 ; 0,579 ; 1,160$ e $1,640 \mathrm{mg} / \mathrm{mL})$, em diferentes temperaturas - (grupo 1). . . . . . . . . . . . . . . . . . . . . . . . . . . . 70

6.5 Gráficos de Zimm para o DMPG $(C=0,255 ; 0,889 ; 1,185$ e $1,660 \mathrm{mg} / \mathrm{mL})$, em diferentes

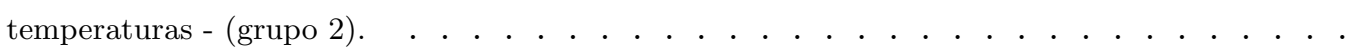

6.6 Gráficos de Zimm para o DMPC $(C=0,285 ; 0,590 ; 0,895$ e 1,566 $\mathrm{mg} / \mathrm{mL})$, em diferentes temperaturas - $($ grupo 1). . . . . . . . . . . . . . . . . . . . . . . . . . . . . . . 72

6.7 Gráficos de Zimm para o $\operatorname{DMPC}(C=0,597 ; 0,942$ e 1, $227 \mathrm{mg} / \mathrm{mL})$, em diferentes tempera-

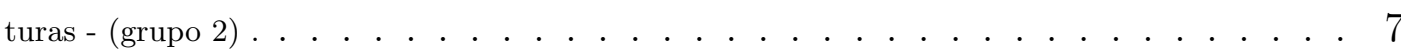

6.8 Comparação do segundo coeficiente do virial obtido para os lipídios DMPG e DMPC, pela análise de Zimm em diferentes temperaturas. . . . . . . . . . . . . . . . . . . . . . . 74

6.9 Comparação do raio de giração obtido para os lipídios DMPG e DMPC, pela análise de Zimm em diferentes temperaturas. . . . . . . . . . . . . . . . . . . . . . . . . . . . 75

6.10 Intensidade da luz espalhada em função da temperatura, para a dispersão lipídica DMPC. • 77

6.11 Intensidade da luz espalhada em função da temperatura, para as dispersão lipídica DMPG. 78

6.12 Cálculo do coeficiente de difusão translacional a partir do coeficiente angular das retas. Cada gráfico representa uma concentração de DMPG em diferentes temperaturas. Esse grupo de amostras foi denominado (grupo 1$)$. . . . . . . . . . . . . . . . . . . . . . . . . . .

6.13 Cálculo do coeficiente de difusão translacional a partir do coeficiente angular das retas. Cada gráfico representa uma concentração de DMPG em diferentes temperaturas. Esse grupo de amostras foi denominado (grupo 2). . . . . . . . . . . . . . . . . . . . 
6.14 Exemplo da reprodutibilidade da medida de DLS para o DMPG. . . . . . . . . . . . . . . 82

6.15 Determinação do coeficiente de difusão no limite de diluição infinita a partir do coeficiente linear das retas. Cada gráfico relaciona os $D_{T}$ das amostras do (grupo 1) de DMPG com as devidas contrações. Essa análise é realizada para as diferentes temperaturas medidas. . . . .

6.16 Determinação do coeficiente de difusão no limite de diluição infinita a partir do coeficiente linear das retas. Cada gráfico relaciona os $D_{T}$ das amostras do (grupo 2) de DMPG com as devidas contrações. Essa análise é realizada para as diferentes temperaturas medidas. . . . . 84

6.17 Cálculo do coeficiente de difusão translacional a partir do coeficiente angular das retas. Cada gráfico representa uma concentração de DMPC em diferentes temperaturas. Esse grupo de amostras foi denominado (grupo 1). . . . . . . . . . . . . . . . . . . . . . . . . . . . . .

6.18 Cálculo do coeficiente de difusão translacional a partir do coeficiente angular das retas. Cada gráfico representa uma concentração de DMPC em diferentes temperaturas. Esse grupo de amostras foi denominado (grupo 2). . . . . . . . . . . . . . . . . . . . . . . . . . . 87

6.19 Exemplo da reprodutibilidade da medida de DLS para o DMPC. . . . . . . . . . . . . . . 88

6.20 Determinação do coeficiente de difusão no limite de diluição infinita a partir do coeficiente linear das retas. Cada gráfico relaciona os $D_{T}$ das amostras do (grupo 1) de DMPC com as devidas contrações. Essa análise é realizada para as diferentes temperaturas medidas. . . . .

6.21 Determinação do coeficiente de difusão no limite de diluição infinita a partir do coeficiente linear das retas. Cada gráfico relaciona os $D_{T}$ das amostras do (grupo 2) de DMPC com as devidas contrações. Essa análise é realizada para as diferentes temperaturas medidas. . . . 90

6.22 Coeficientes de difusão no regime de diluição infinita, em função da temperatura, para o DMPG (esquerda) e DMPC (direita). . . . . . . . . . . . . . . . . . . . . . . . . . . . . . 91

6.23 Diâmetro efetivo, obtido dos resultados DLS a partir da relação de Stokes-Einstein, no regime de diluição infinita, em função da temperatura, para o DMPG (esquerda) e DMPC (direita). .

6.24 Comparação entre os raios efetivos obtidos por SLS e DLS para o DMPG e DMPC, em função da temperatura. . . . . . . . . . . . . . . . . . . . . . . . . . . . . . . . 93

6.25 Medidas de anisotropia dos centros espalhadores. . . . . . . . . . . . . . . . . . . . . 97

6.26 Ilustração do cálculo do número de lipídios e do número de poros. O número de lipídios é preservado constante na fase gel, fluida e na região de transição, portanto o número de lipídios em excesso será proporcional ao número de poros, dado pelos sítios em preto. . . . . . . . . 100

6.27 Modelo da deformação do DMPG, na região de transição. . . . . . . . . . . . . . . . . 102

B.1 Exemplo das função de correlação do campo elétrico e o ajuste obtido pelo método do programa Gendist, para o DMPG, onde $\tau$ é dado em $\mu$ s. . . . . . . . . . . . . . . . . . . . . . . 114 
B.2 Distribuição de tamanhos obtida pelo método do programa Gendist, DMPG $C=0,34 \mathrm{mM}$. . 114

B.3 Exemplo das função de correlação do campo elétrico e o ajuste obtido pelo método do programa

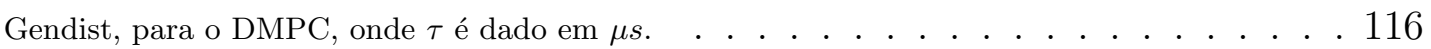

B.4 Distribuição de tamanhos obtida pelo método do programa Gendist, DMPC $C=0,49 m M . \quad$. 116

C.1 Comparação do diâmetro efetivo do DMPG não extrusado com o DMPG extrusado (11 e 31 vezes), obtidos por DLS. . . . . . . . . . . . . 120 



\section{Lista de Tabelas}

5.1 Concentrações obtidas da dosagem de fosfato, em $(\mathrm{mM})$ e em $(\mathrm{mg} / \mathrm{mL})$, para dois conjuntos de amostras de DMPG. . . . . . . . . . . . . . . . . . . . . . . . . . . . . . 52

5.2 Concentrações obtidas da dosagem de fosfato, em $(\mathrm{mM})$ e em $(\mathrm{mg} / \mathrm{mL})$, para dois conjuntos de amostras de DMPC. . . . . . . . . . . . . . . . . . . . . . . . . . . . . . . 52

5.3 Incremento no índice de refração para o DMPG, na fase gel, na região de transição e na fase fluída. . . . . . . . . . . . . . . . . . . . . . . . . . . . 57

5.4 Incremento no índice de refração para o DMPC, na fase gel, na transição de fase e na fase fluída. 60

6.1 Resultados dos gráficos de Zimm para o DMPG $(C=0,214 ; 0,579 ; 1,160$ e $1,640 \mathrm{mg} / \mathrm{mL})$,

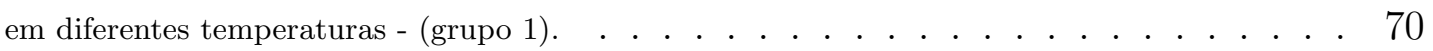

6.2 Resultados dos gráficos de Zimm para o DMPG $(C=0,255 ; 0,889 ; 1,185$ e $1,660 \mathrm{mg} / \mathrm{mL})$,

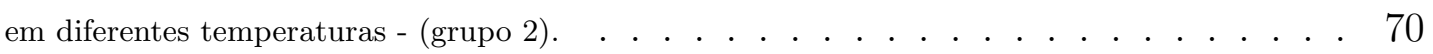

6.3 Resultados dos gráficos de Zimm para o DMPC $(C=0,285 ; 0,590 ; 0,895$ e 1, $566 \mathrm{mg} / \mathrm{mL})$, em diferentes temperaturas - $($ grupo 1$) . \quad$. . . . . . . . . . . . . . . . . . . . . . . . 73

6.4 Resultados dos gráficos de Zimm para o DMPC $(C=0,597 ; 0,942$ e $1,227 \mathrm{mg} / \mathrm{mL})$, em

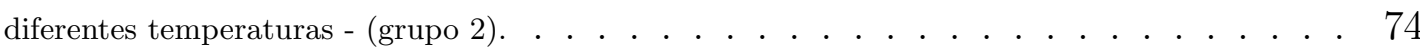

6.5 Raio efetivo do DMPG calculado numericamente para vesículas a partir do $R_{g}$ obtido pela análise de Zimm em diferentes temperaturas. . . . . . . . . . . . . . . . . . . . . . . 76

6.6 Raio efetivo do DMPC calculado numericamente para vesículas a partir do $R_{g}$ obtido pela análise de Zimm em diferentes temperaturas. . . . . . . . . . . . . . . . . . . . . . . 77

6.7 Coeficiente de difusão e diâmetro efetivo do DMPG no regime de diluição infinita - grupo $1 . \quad$ • 83

6.8 Coeficiente de difusão e diâmetro efetivo do DMPG no regime de diluição infinita - grupo $2 . \quad$ • 84

6.9 Média do índice de polidispersão do DMPG obtida pelo método dos Cumulantes de segunda ordem. . . . . . . . . . . . . . . . . . . . . . . . . . . . 85

6.10 Coeficiente de difusão e diâmetro efetivo do DMPC no limite de diluição infinita - grupo $1 . \quad$ • $\quad 89$

6.11 Coeficiente de difusão e diâmetro efetivo do DMPC no regime de diluição infinita - grupo $2 . \quad$ • 90 
6.12 Média do índice de polidispersão do DMPG obtida pelo método dos Cumulantes de segunda ordem). . . . . . . . . . . . . . . . . . . . . . . . . . . . . . . . . . . . . . . 91

6.13 Raio efetivo obtido a partir da média das medidas realizadas por DLS e SLS para o DMPG. $\quad .94$

6.14 Raio efetivo obtido a partir da média das medidas realizadas por DLS e SLS para o DMPC. $\quad 94$

6.15 Razão entre a área ocupada por cabeça polar de lipídio na fase gel e fluida. . . . . . . . . . 95

6.16 Valores utilizados para estimar o número de lipídios necessários para construir uma vesícula de $R_{e f}$. . . . . . . . . . . . . . . . . . . . . . . . . . . . 100

6.17 Porcentagem da área ocupada por buracos variando a $A_{C P L}$. . . . . . . . . . . . . . . . 101

6.18 Concentração de contra-íons no volume externo às vesículas e no volume interno das vesículas. 105

B.1 Comparação dos métodos Cumulantes segunda ordem e Gendist, para o DMPG $C=0,34 m M .115$

B.2 Comparação dos métodos Cumulantes ( $2^{a}$ ordem) e Gendist, para o DMPC $C=0,49 m M$. . . 116

C.1 Diâmetro efetivo do DMPG após diferentes números de extrusões. . . . . . . . . . . . . . 121

C.2 Diâmetro efetivo de misturas de esferas de poliestireno de diferentes tamanhos, (Nomura, Enoki

e Lamy - trabalho em fase de conclusão). . . . . . . . . . . . . . . . . . . . . . . . . 121 


\section{Capítulo 1}

\section{Introdução}

As bicamadas lipídicas fornecem à membrana um caráter estrutural. As moléculas lipídicas constituem cerca de 50\% em massa das membranas naturais, sendo o restante constituído praticamente de proteínas. Os lipídios são moléculas anfifílicas, que possuem uma cabeça polar hidrofílica e uma cauda hidrocarbônica hidrofóbica. Membranas modelo podem ser constituídas apenas por lipídios, que se organizam em meio aquoso em estruturas energeticamente favoráveis denominadas lipossomos, que geralmente são muito estáveis. Devido à propriedade de se assemelharem a membranas biológicas, os lipossomos são muito utilizados em estudos físico-químicos e bioquímicos, mimetizando membranas naturais, (Alberts et al., 2000).

Sistemas lipídicos podem apresentar uma transição de fase caracterizada pela ordem e desordem do sistema. Em baixas temperaturas, as cabeças polares dos lipídios seriam arranjadas em uma rede triangular (Janiak, D.M., e Shipley, 1979), e suas cadeias laterais assumiriam conformações estendidas e altamente ordenadas. Em altas temperaturas, as cabeças polares não se organizam em uma rede ordenada e suas cadeias hidrofóbicas não apresentam ordem lateral. Como, por exemplo, podemos observar o dimiristoil fosfatidil colina, (DMPC). Observe a figura 1.1 extraída de (Heimburg, 2007).

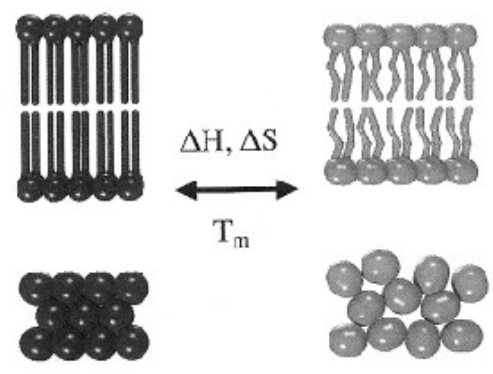

Figura 1.1: Transição ordem-desordem dos lipídios. 
Deste modo, os lipídios podem apresentar duas fases distintas denominadas fase gel e fase fluida, (Heimburg, 2007). A transição entre essas duas fases distintas é altamente cooperativa apresentando um pico bem pronunciado e estreito na Capacidade térmica, na temperatura de transição.

Muitos lipídios apresentam uma pré-transição, bem menos cooperativa e com menor entalpia. Experimentos de microscopia eletrônica e microscopia de força atômica sugerem que a pré-transição seja a transição de uma fase gel altamente ordenada, para uma fase denominada "ripple" onde a bicamada apresenta ondulações periódicas, mas ainda é bastante ordenada quando comparada à fase fluida (Heimburg, 2007) (observe a figura $1.2)$.

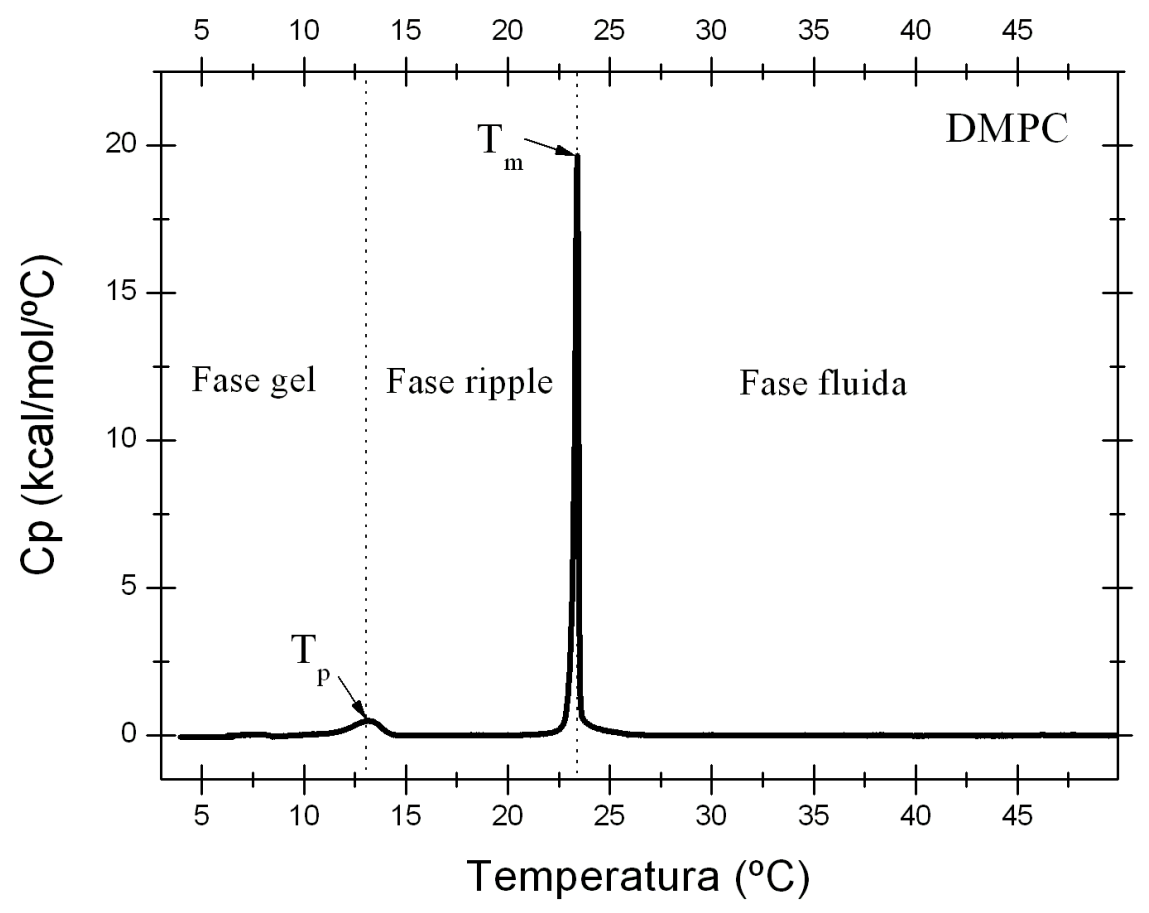

Figura 1.2: Transição de fase altamente cooperativa do lipídio DMPC.

Neste trabalho chamaremos de fase gel toda a região que precede a transição principal, englobando portanto, as chamadas fase gel e fase ripple.

A proposta desse trabalho é estudar um lipídio aniônico em particular. Sob condições fisiológicas, muitas membranas celulares são negativamente carregadas devido à presença de lipídios com cabeças polares acídicas. O fosfatidil glicerol (PG) é o fosfolipídio aniônico mais abundante encontrado nas membranas celulares procarióticas, e tem sido estudado e utilizado como modelo para membranas biológicas, ou regiões de membranas negativamente carregadas. 
O dimiristoil fosfatidil glicerol (DMPG), lipídio saturado com 14 carbonos nas duas cadeias hidrocarbônicas, em condições fisiológicas (alta força iônica $300 \mathrm{mM}$ ) apresenta uma transição de fase gel-fluido altamente cooperativa, em torno de $23^{\circ} \mathrm{C}$, como podemos observar na figura 1.3.

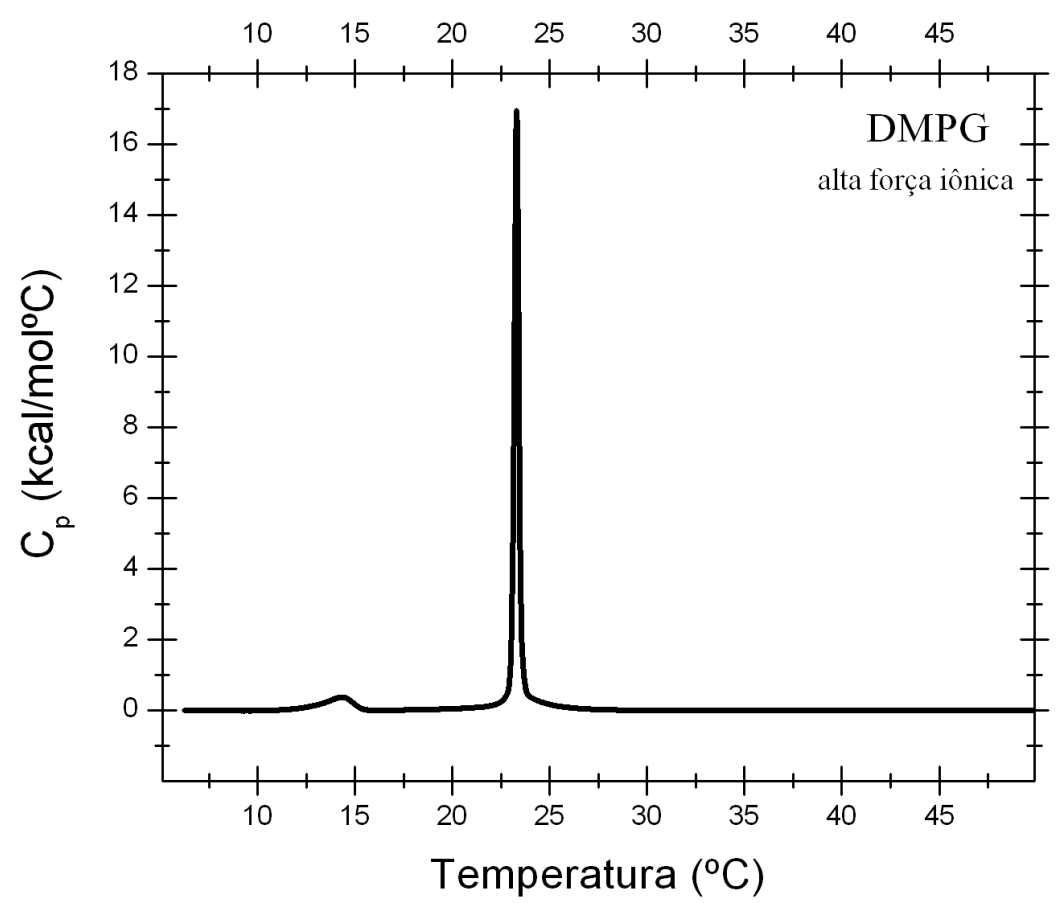

Figura 1.3: Comportamento termo-estrutural do DMPG a alta força iônica, $[\mathrm{NaCl}]=500 \mathrm{mM}$, gráfico cedido pelo aluno de doutorado Barroso, R.P.

No entanto, em baixa força iônica, foi visto que dispersões de DMPG exibem um comportamento termo-estrutural muito peculiar e interessante (Salonen, Eklund, Virtanen, e Kinnunen, 1989; Heimburg e Biltonen, 1994; Riske, Politi, Reed, e Lamy-Freund, 1997; Riske, Nascimento, Peric, Bales, e Lamy-Freund, 1999; Riske, Amaral, e Lamy-Freund, 2001; Riske, Fernandez, Nascimento, Bales, e Lamy-Freund, 2003; Riske, Amaral, Döbereiner, e Lamy, 2004; Fernandez, Riske, Amaral, e Lamy, 2008; Lamy-Freund e Riske, 2003; Alakoskela e Kinnunnen, 2007; Barroso, Riske, Henriques, e Lamy, 2010), apresentando uma "região de transição de fase", entre aproximadamente $18\left(T_{m}^{o n}\right)$ e $37^{\circ} C\left(T_{m}^{o f f}\right)$, observe a figura 1.4. Em $T_{m}^{o n}$ aparece um pico estreito de absorção de calor, seguido por outros mais largos, sendo o último em $T_{m}^{o f f}$. Marcadores de spin e fluorescentes, incorporados à bicamada lipídica, mostraram uma mudança contínua no empacotamento da membrana entre $T_{m}^{o n}$ e $T_{m}^{o f f}$. Entretanto, com alguns marcadores de spin, é possível observar, nitidamente, a existência de uma queda mais acentuada no empacotamento da 
bicamada no início da transição, em $T_{m}^{o n}$. Os resultados da literatura também evidenciam variações bruscas de turbidez da amostra, com diminuição em $T_{m}^{o n}$, e aumento em $T_{m}^{o f f}$, sendo que a dispersão de DMPG é visualmente transparente na região de transição. Também muito peculiares são a alta viscosidade apresentada pela dispersão de DMPG nesta região de transição, e a alta condutividade (Salonen et al., 1989; Riske et al., 1997; Barroso et al., 2010). Além do baixo espalhamento de luz, esta região também apresenta um baixo espalhamento de raios-X a baixo ângulo (SAXS). Essa técnica detectou a presença de um pico (não muito estreito) de interferência a muito baixo ângulo, correspondendo a uma distância de repetição da ordem de $400 \AA$.

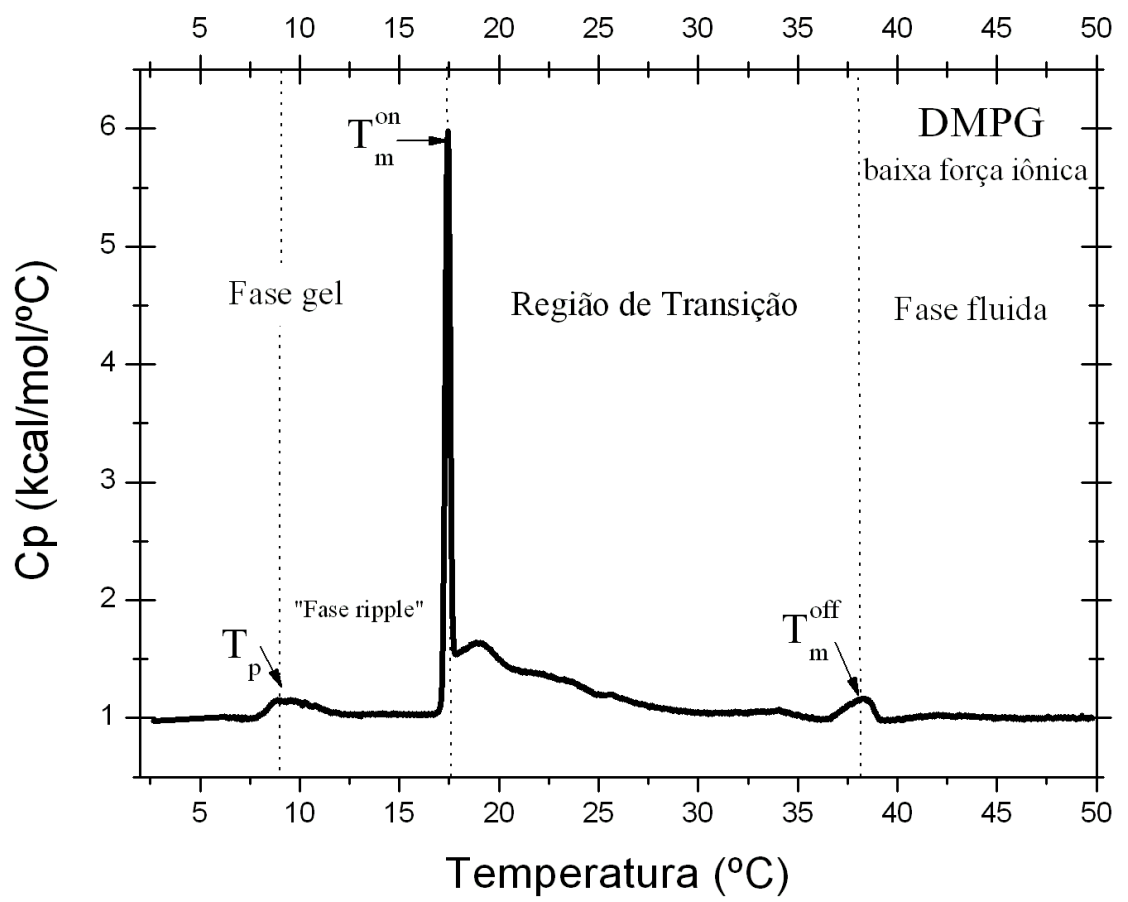

Figura 1.4: Comportamento termo-estrutural do DMPG $(1,0 m M)$ em baixa força iônica.

Outras características importantes são observadas na região de transição. Espectros de ressonância paramagnética eletrônica (RPE) de marcadores de spin derivados de fosfolipídio, marcados no carbono 16 da cadeia acila (16-PCSL), incorporados na bicamada, indicaram a presença de dois sinais de RPE, sugerindo a coexistência de dois domínios com microviscosidades diferentes: um deles típico de bicamada, e outro altamente fluido e hidratado, semelhante a anfifílicos em micelas, possivelmente em sítios de alta curvatura (Riske et al., 2003). Para temperaturas acima de $T_{m}^{o f f}$, a bicamada reorganiza-se em uma fase fluida convencional. A observação de vesículas gigantes (GUVs) de DMPG, com microscopia por contraste de fase, em baixa força iônica, indica seu "desaparecimento" 
quando a amostra é esfriada, saindo da fase fluida e entrando na região de transição (Riske et al., 2004). Entretanto, a vesícula não se desfaz, pois ela reaparece na mesma posição quando a temperatura da amostra é aumentada, acima de $T_{m}^{o f f}$, voltando à fase fluida. Essa observação sugere que a baixa turbidez poderia estar relacionada à perda de contraste óptico da bicamada com o meio. É importante ressaltar que não ocorre rearranjo de lipídios entre as estruturas formadas pelo DMPG ao longo da região de transição de fase, (Lamy-Freund e Riske, 2003).

Considerando o aumento da condutividade elétrica observado na região de transição, foi proposto que a causa esteja relacionada a um aumento na concentração de $\mathrm{Na}^{+}$em solução, consequência do aumento do grau de ionização dos lipídios nessa região, (Riske et al., 1997; Barroso et al., 2010), sugerindo uma diminuição da constante de ligação $N a^{+}-P G^{-}$entre $T_{m}^{o n}$ e $T_{m}^{o f f}$.

Para a verificação do aumento do grau de ionização dos lipídios na região de transição, foi feito um estudo da variação do potencial de superfície dos agregados de DMPG com a temperatura, usando um marcador de spin catiônico (CAT-1), aquo-solúvel, (Riske et al., 2003). O potencial eletrostático de superfície foi calculado através da partição deste marcador catiônico entre a solução aquosa e a superfície aniônica dos agregados de DMPG. Entretanto, por problemas de interpretação dos espectros de RPE, o possível aumento do potencial de superfície somente na região de transição, entre as fases gel e fluida, não pôde ser constatado. Como complementação do trabalho citado, foi feito um estudo teórico, baseado no modelo esférico de campo médio de Poisson-Boltzman, onde se investigou a dependência do potencial de superfície de esferas coloidais carregadas com a concentração do anfifílico, força iônica e pH do meio. Considerando os dados experimentais obtidos com o marcador de spin CAT-1, concluiu-se que era necessária uma ligação específica entre o marcador e a superfície da bicamada, (Tamashiro, Henriques, e Lamy, 2005).

A região de transição pode estar associada a diferentes fatores estruturais do DMPG. Observou-se que o aumento da força iônica leva a uma diminuição da região de transição tal como o aumento da concentração de DMPG, (Riske et al., 1997; Barroso et al., 2010). Isso pode estar associado à disponibilidade de íons em solução. O curto comprimento da cadeias carbônicas também é um fator que pode estar relacionado com a existência dessa região: podemos observar na figura 1.5 à direita (Heimburg, 2007) que a diminuição da cadeia carbônica leva ao alargamento da transição principal e ao surgimento de picos menores na capacidade térmica.

A temperatura é uma variável importante para desencadear um processo de mudanças 
estruturais nos lipídios, pois após a transição principal os lipídios apresentam maior mobilidade e a área ocupada por cabeça polar de lipídio aumenta, (Marsh e Phil, 1990; Nagle e Tristram-Nagle, 2000), permitindo inclusive maior hidratação pelo solvente,

Várias das características da região de transição discutidas acima levaram a propostas de modelos possíveis para o sistema. Os modelos contemplam diferentes características observadas.

Heimburg apresenta uma discussão na literatura para o DMPG, sugerindo que a mudança drástica da viscosidade, indica a formação de uma fase bicontínua, (Heimburg, 2007) (observe a figura 1.6 à esquerda (Heimburg, 2007)).

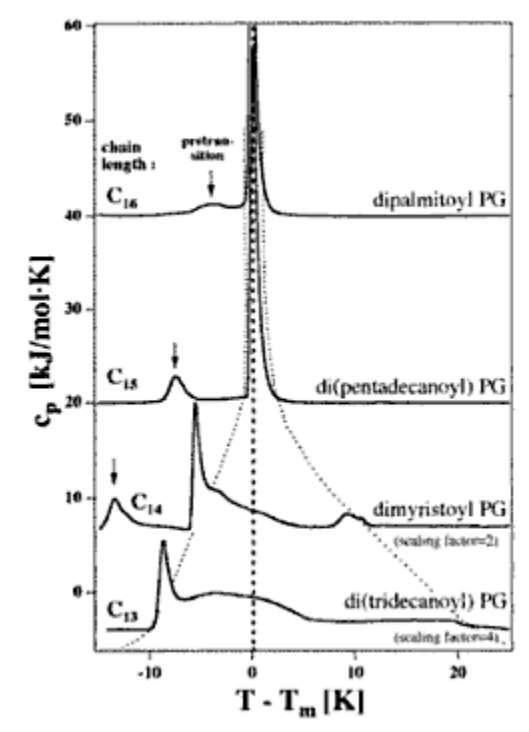

Figura 1.5: Aumento da região de transição para cadeias carbônicas curtas.

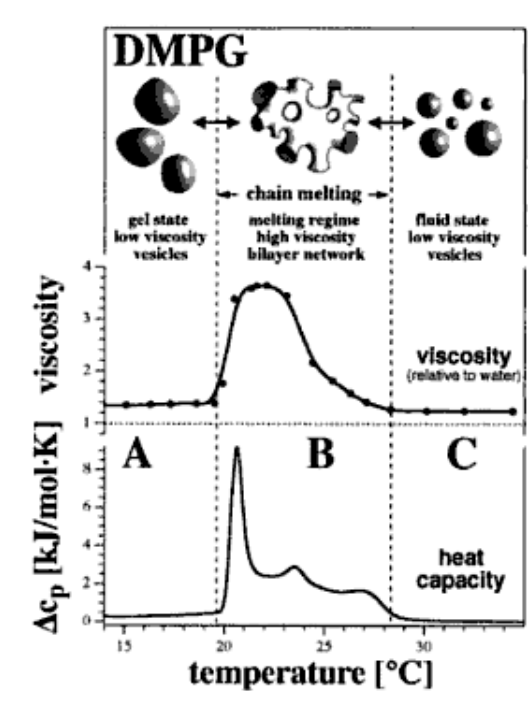

Figura 1.6: Aumento da viscosidade relativa relacionada com a capacidade térmica e mudança estrutural na região de transição do DMPG.

Entretanto Heimburg sugere que na região de transição há fusão de agregados lipídicos (figura 1.5 à esquerda, (Heimburg, 2007)) o que está em desacordo com o que foi visto em (Lamy-Freund e Riske, 2003; Alakoskela e Kinnunnen, 2007).

Em outro modelo considerando os dados de espalhamento de luz, condutividade e viscosidade, (Riske et al., 1997), foi proposto a existência de pequenas vesículas de DMPG agregadas nas fases gel e fluida, que desagregariam na região de transição. A proposta sugere que na região de transição as pequenas vesículas estariam mais carregadas por isso a repulsão eletrostática seria maior. A ideia foi desenvolvida posteriormente em um modelo estatístico (Goldman, 2001; Goldman, Riske, e Lamy-Freund, 1999). Neste modelo teórico, as vesículas foram representadas por partículas sem carga, e estudou-se as propriedades estatísticas de dispersões de agregados, levando em consideração tanto os graus de 
liberdade internos, expressos, por exemplo, através de flutuações na forma dos agregados, bem como algumas propriedades relevantes da dispersão, tal como índice de polidispersão. Os resultados obtidos para a termodinâmica de um sistema modelo, com base em regras de soma formuladas adequadamente para a densidade de anfifílicos (Goldman et al., 1999), incluindo a presença de sal (Goldman, 2001), sugeriram que a estatística da dispersão poderia ser, de fato, determinante no estado dos anfifílicos agregados. Apesar de não apresentar transições de fase, o modelo foi capaz de interpretar, qualitativamente, dados obtidos por espalhamento de luz e SAXS em dispersões de DMPG a baixa força iônica (Goldman et al., 1999). Neste modelo, aparece uma distribuição de partículas de diferentes tamanhos, dependente da temperatura. Relativo ao pico de Bragg mencionado acima, discutiu-se também a possibilidade das partículas menores serem pequenos fragmentos de bicamada, bicelas, e o pico de Bragg estar relacionado à distância entre bicelas. Entretanto, este modelo não leva em conta as características específicas do DMPG, e as cargas de superfície, e portanto, a repulsão eletrostática entre os agregados.

Em um terceiro modelo, qualitativo, foi sugerido a possibilidade da ocorrência de "membranas perfuradas" para a região de transição do DMPG em baixa força iônica (Riske et al., 2004; Spinozzi, Paccamiccio, Mariani, e Amaral, 2010). Este modelo está de acordo com a existência de regiões de diferentes empacotamentos na membrana (Riske et al., 2003) (a região de bicamada, e os poros, menos empacotados e mais hidratados), a não reorganização dos agregados lipídicos na região de transição (Lamy-Freund e Riske, 2003; Alakoskela e Kinnunnen, 2007). A presença do pico de Bragg relativo a uma distância de repetição $400 \AA$ (Riske et al., 2004; Spinozzi et al., 2010) foi interpretada como a distância de repetição dos poros.

No entanto não há um modelo que contemple todos os resultados experimentais, sendo necessário maiores investigações.

Tendo em vista os possíveis tamanhos dos agregados de DMPG em meio aquoso, 50 $500 \mathrm{~nm}$, a técnica experimental Espalhamento de luz ( $\lambda \sim 633 \mathrm{~nm}$ ) mostra-se adequada para a avaliação das dimensões destes agregados e o estudo do índice de polidispersão da dispersão aquosa. Portanto, neste trabalho vamos estudar dispersões lipídicas de DMPG e de DMPC (dimiristoil fosfatidil colina) por espalhamento de luz. O DMPC foi utilizado como controle, pois possui as mesmas cadeias hidrocarbônicas e diferente cabeça polar, neutra.

A intensidade de luz espalhada por uma amostra pode ser medida, de maneira simples, com o auxílio de um espectrofotômetro, através da medida de turbidez da amostra. 
Entretanto, para a obtenção de informações sobre o tamanho e forma da partícula espalhadora, índice de polidispersão, massa molecular, e interações entre partículas, são necessários medidas mais sofisticadas, como por exemplo a análise de Zimm e medidas de correlações temporais. O espalhamento de luz possui duas abordagens diferenciadas. No Espalhamento de Luz Estático (SLS), a intensidade da luz espalhada é coletada em função do ângulo de espalhamento. No Espalhamento de Luz Dinâmico (DLS), a correlação da intensidade da luz espalhada é obtida em função do tempo, para um dado ângulo fixo. As duas análises fornecem informações complementares a respeito do sistema.

Para o espalhamento de luz estático, usa-se a teoria de espalhamento de luz de Rayleigh, com as correções de Debye para flutuações de densidade em líquidos, e para a interferência intra-partícula, explicitada no fator de forma da partícula, como podemos ver no Capítulo 2. Através do método desenvolvido por Zimm (1948), sendo a luz espalhada medida a vários ângulos, e em uma faixa de concentrações de soluto, podemos obter parâmetros que nos informam sobre a forma, tamanho e interações dos centros espalhadores em solução. Tais parâmetros são: o segundo coeficiente do virial, $A_{2}$, a massa molecular média, $\bar{M}_{w}$ e o raio de giração da partícula.

O espalhamento de luz dinâmico surgiu na década de 60, quando se observou que as flutuações da intensidade de luz espalhada no tempo podiam ser usadas para obter informações sobre o movimento browniano de partículas dispersas ou macromoléculas em solução. Considerando que a dinâmica dos agregados presentes nas dispersões possa ser descrita por processos difusivos, a função de autocorrelação temporal pode ser tratada pelo método dos cumulantes, (Koppel, 1972). Esse método descreve o decaimento da função de correlação do campo elétrico por uma taxa de decaimento média, $\Gamma$, que está relacionada a uma frequência de relaxação do movimento da partícula, onde $\Gamma$ é diretamente proporcional ao coeficiente de difusão dos centros espalhadores. O método também descreve uma dispersão da média, a qual se relaciona ao índice de polidispersão do sistema. Além disso, por meio do coeficiente de difusão, é possível obter um tamanho médio dos agregados em solução, de acordo com a equação de Stokes-Einstein, conforme podemos observar no Capítulo 3. 


\section{Capítulo 2}

\section{Espalhamento de luz estático: Uma abordagem diferenciada}

\subsection{Introdução}

Este capítulo é destinado em especial ao espalhamento de luz estático (SLS). A discussão apresentada aqui baseia-se em uma apostila idealizada pela Prof. ${ }^{a}$ Vera B. Henriques, em colaboração com o aluno de doutorado, Tiago R. Olivera e Prof. ${ }^{a}$ Maria Teresa Lamy. Esta apostila que está em fase de conclusão, e aborda a teoria de espalhamento de luz estático do ponto de vista da mecânica estatística. A elaboração, discussão, alguns resultados experimentais e teóricos desta apostila, fizeram parte dos objetivos deste mestrado. Neste capítulo, em particular, o objetivo principal é obter a equação de Zimm.

A fase líquida da matéria se distingue da fase gasosa por apresentar estrutura local. Podemos investigar o estado desta estrutura local através do espalhamento de ondas eletromagnéticas. Embora em líquidos as moléculas não apresentem posições de equilíbrio constantes no tempo, é possível obter informações sobre o arranjo médio das moléculas em solução, ou seja como as moléculas se organizam determinando uma certa estrutura. A geometria das moléculas e suas interações serão responsáveis por diferentes interferências impostas à intensidade da luz espalhada. 


\subsection{Espalhamento elástico de uma carga pontual}

O espalhamento elástico é uma aproximação que admite que a frequência da radiação incidente é igual a frequência da radiação espalhada. No caso de elétrons livres, a conservação da energia e do momento dá origem ao chamado efeito Compton, (Nussenzveig, 2008), segundo o qual

$$
\frac{w_{i}}{w}-1=\frac{h w_{i}}{m c^{2}}(1-\cos (\theta)),
$$

onde $w_{i}$ e $w$ correspondem a frequência de oscilação da radiação incidente e espalhada, respectivamente, $h$ refere-se a constante de Planck, $c$ corresponde a velocidade da luz e $\theta$ ao ângulo de espalhamento. No espalhamento elástico a frequência da radiação incidente $w_{i}$ é aproximadamente a frequência da radiação reemitida, $w, w_{i} \approx w$, pois $1 / w>>h / m c^{2}$, que é válida no caso da luz visível.

Inicialmente, vamos considerar um elétron ligado, oscilando com frequência natural $\omega_{0}$, observe a figura 2.1. Um campo elétrico, representado por uma onda plana $\vec{E}=$

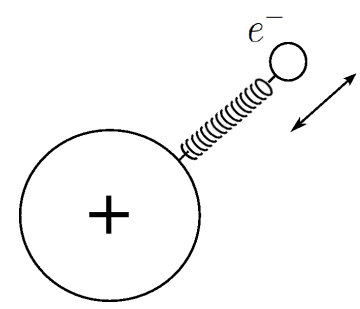

Figura 2.1: Ilustração do modelo: elétron ligado oscilando com frequência natural $\omega_{0}$.

$\vec{E}_{0} \exp (i(\vec{k} \cdot \vec{r}-\omega t))$, atua sobre este elétron (oscilação forçada), que passa a oscilar e a emitir uma onda esférica de mesma frequência (espalhamento elástico), ver por exemplo (Fenkel, 2005).

$$
\ddot{\vec{r}}+\Gamma^{\prime} \dot{\vec{r}}+\omega_{0}^{2} \vec{r}=-\frac{e}{m} \vec{E}_{0} \exp (-i \omega t)
$$

onde $\Gamma^{\prime}$ corresponde a um coeficiente de resistência. A solução estacionária desta equação é dada por

$$
\vec{r}=-\frac{e}{m}\left\{\frac{1}{\omega^{2}-\omega_{0}^{2}+i \Gamma^{\prime} \omega}\right\} \vec{E}_{0} e^{-i(\omega t)}
$$

Portanto

$$
\ddot{\vec{r}}(t)=\frac{e}{m}\left\{\frac{\omega^{2}}{\omega^{2}-\omega_{0}^{2}+i \Gamma^{\prime} \omega}\right\} \overrightarrow{E_{0}} e^{-i(\omega t)},
$$

A amplitude da onda espalhada deve depender, em geral, da frequência natural do elétron $\omega_{0}$, e da frequência do campo elétrico incidente $\omega$. O campo elétrico da onda 
espalhada, centrada na posição de equilíbrio do elétron, é dado por

$$
\vec{E}(\theta, \omega, t, R)=\frac{e \prod(\theta) \ddot{\vec{r}}(t-R / c)}{4 \pi \epsilon_{0} c^{2} R}
$$

com a aceleração $\ddot{\vec{r}}(t-R / c)$ dada pela equação $(2.4)$.

$R$ corresponde à posição de observação (detector), e $\Pi(\theta)$ está relacionado à polarização incidente.

A intensidade da luz espalhada é dada por

$$
I=\frac{\left\langle\epsilon c E^{2}\right\rangle}{2}
$$

ou ainda

$$
I=\frac{e^{4} \Pi(\theta)}{2 m^{2}(4 \pi)^{2} \epsilon_{0} c^{3} R^{2}}\left\{\frac{w^{4} E_{0}^{2}}{\left(w^{2}-w_{0}^{2}\right)^{2}+(\Gamma w)^{2}}\right\},
$$

onde $\Pi^{2}(\theta)=1+\cos ^{2}(\theta)$, para a luz não polarizada. ${ }^{1}$

Quando a frequência de radiação incidente é muito maior que a frequência natural dos elétrons $\left(\omega>>\omega_{0}\right)$, temos o chamado espalhamento Thompson, onde a intensidade da luz espalhada não depende da frequência da radiação incidente. Por outro lado, quando $\omega<<\omega_{0}$, no espalhamento Rayleigh, a intensidade da luz espalhada é proporcional ao inverso do comprimento de onda à quarta potência, $I(w) \sim \omega^{4} / \omega_{0}^{4} \sim 1 / \lambda^{4}$, ver por exemplo (Nussenzveig, 2008).

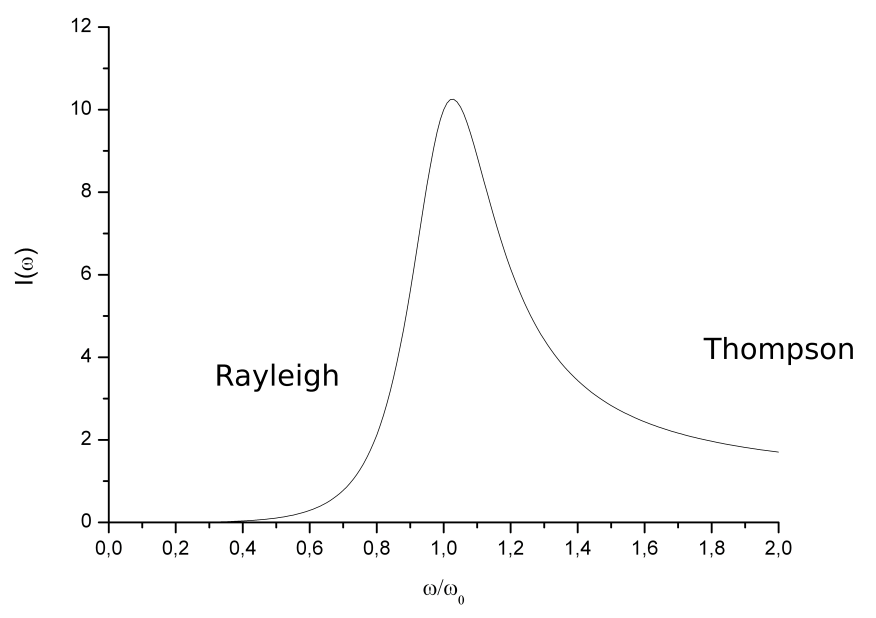

Figura 2.2: Intensidade da luz espalhada em função da frequência de oscilação $w$ e da frequência natural do elétron $\omega_{0}$.

\footnotetext{
${ }^{1}$ Para a luz verticalmente polarizada, $\Pi^{2}(\theta)=1$
} 


\subsection{Interferência das ondas espalhadas por vários elétrons}

Vamos considerar agora duas cargas pontuais separadas por uma distância $d$, conforme mostra a Figura 2.3. O campo elétrico da onda incidente tem valor $E_{1}=E_{0} \exp \left(i\left(\vec{k} \cdot \vec{r}-\omega t_{1}\right)\right)$ quando excita o elétron 1 no instante $t_{1}$, e $E_{2}=E_{0} \exp \left(i\left(\vec{k} \cdot \vec{r}-\omega t_{2}\right)\right)$ quando excita o elétron 2 no instante $t_{2}$. Para um mesmo instante de tempo $t$, as ondas espalhadas pelos elétrons 1 e 2 possuem uma defasagem tal que

$$
t_{1}=t \quad, \quad t_{2}=t-\frac{d}{c}
$$

O espalhamento é instantâneo, portanto para que a onda espalhada pelo elétron 1 interfira com a onda espalhada pelo elétron 2 , é necessário que esta última seja emitida em um instante de tempo $t_{2}$ anterior a $t_{1}$. Pois como ilustra a figura 2.3, o elétron 2 está mais distante do ponto de partida das ondas incidentes.

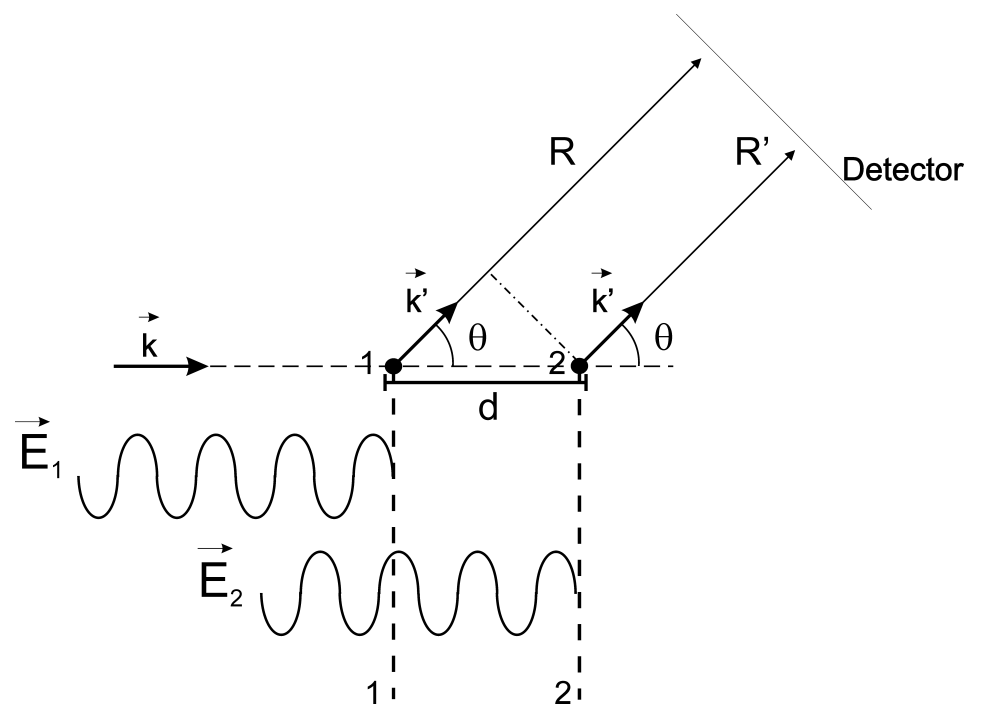

Figura 2.3: Interferência da luz espalhada por duas partículas.

O campo elétrico da onda espalhada que atinge o detector deve ser então proporcional a

$$
B_{1}(\theta, \omega) \vec{E}_{0} \frac{\exp \left(i \vec{k}^{\prime} \cdot \vec{R}\right)}{R} \exp (-i \omega t)+B_{2}(\theta, \omega) \vec{E}_{0} \frac{\exp \left(i \vec{k}^{\prime} \cdot \vec{R}^{\prime}\right)}{R^{\prime}} \exp \left[-i \omega\left(t-\frac{d}{c}\right)\right],
$$

onde

$$
B(\theta, \omega) \equiv \frac{e \Pi(\theta)}{4 \pi \epsilon_{0} c^{2}} \frac{e}{m}\left\{\frac{1}{\omega^{2}-\omega_{0}^{2}+i \Gamma \omega}\right\} .
$$

Para cargas elétricas idênticas, $B_{1}=B_{2}$, de forma que podemos escrever o campo elétrico espalhado

$$
\vec{E}=B(\theta, \omega) \vec{E}_{0} \frac{\exp \left[i\left(k^{\prime} R-\omega t\right)\right]}{R}\left\{1+\frac{\exp \left[i k^{\prime}\left(R^{\prime}-R\right)\right]}{R^{\prime} / R} \exp \left(i k^{\prime} d\right)\right\} .
$$


A diferença de caminho $\vec{R}-\vec{R}^{\prime}=d \cos \theta$, como mostra a Figura 2.3. Assim

$$
\frac{R}{R^{\prime}}=\frac{d \cos \theta+R^{\prime}}{R^{\prime}}=1+\frac{d \cos (\theta)}{R} \approx 1
$$

para $d<<R$. Portanto, neste limite

$$
\vec{E}=B(\theta, \omega) \vec{E}_{0} \frac{\exp \left(i\left(k^{\prime} R^{\prime}-\omega t\right)\right)}{R^{\prime}}\left\{1+\exp \left[i k^{\prime} d(1-\cos \theta)\right]\right\}
$$

Podemos escrever a expressão anterior em termos das posições relativas dos elétrons. Por exemplo, vamos definir as posições dos elétrons 1 e 2 como $\vec{r}_{1}=(d, 0,0)$ e $\vec{r}_{2}=(0,0,0)$.

Observe que

$$
d=\frac{\vec{k} \cdot \vec{r}_{1}}{k} \Rightarrow \quad k^{\prime} d=k^{\prime} \frac{\vec{k} \cdot \vec{r}_{1}}{k} \underset{k^{\prime}=k}{=} \vec{k} \cdot \vec{r}_{1}
$$

e

$$
\overrightarrow{k^{\prime}} \cdot \vec{r}_{1}=k^{\prime} d \cos \theta
$$

$\log \mathrm{O}$

$$
k^{\prime} d(1-\cos \theta)=\left(\vec{k}-\vec{k}^{\prime}\right) \cdot \vec{r}_{1}
$$

Como centramos a partícula 2 na origem, $\exp \left[\left(\vec{k}-\vec{k}^{\prime}\right) \cdot \vec{r}_{2}\right]=1$, e a equação $(2.10)$ pode ser reescrita na forma

$$
\vec{E}=B(\theta, \omega) \vec{E}_{0} \frac{\exp \left(i\left(k^{\prime} R^{\prime}-\omega t\right)\right)}{R^{\prime}} \sum_{i=1}^{N=2} \exp \left(i\left(\vec{k}-\vec{k}^{\prime}\right) \cdot \vec{r}_{i}\right) .
$$

A equação (2.11) pode ser estendida para o caso de uma linha de partículas, ou mesmo para situações gerais, qualquer ângulo de incidência ou qualquer distribuição de partículas, desde que a aproximação $d<<R$ não seja violada. Quando observamos um conjunto de partículas espalhadoras, a distância $R$ passa a representar a distância do detector ao volume de espalhamento.

O vetor de espalhamento é definido por $\vec{q} \equiv \vec{k}^{\prime}-\vec{k}$ e pode ser escrito segundo a forma

$$
q=2 k \sin (\theta / 2)
$$

Portanto, a intensidade da luz espalhada, obtida da equação (2.6) é dada por

$$
I(\theta, \omega)=\underbrace{\frac{c \epsilon_{0}}{2} E_{0}^{2}}_{I_{0}} \frac{|B(\theta, \omega)|^{2}}{R^{2}} \sum_{i=1}^{N} \sum_{j=1}^{N} \exp \left(i \vec{q} \cdot\left(\vec{r}_{i}-r_{j}\right)\right) .
$$

onde,

$$
|B(\theta, \omega)|^{2}=\frac{e^{4}\left(1+\cos ^{2}(\theta)\right)}{m^{2}\left(4 \pi \epsilon_{0} c^{2}\right)^{2}}\left\{\frac{\omega^{4}}{\left(\omega^{2}-\omega_{0}^{2}\right)^{2}-\Gamma \omega}\right\}
$$




\subsection{Contribuição eletrônica de diferentes átomos}

Podemos separar a contribuição dos diferentes elétrons associados a cada átomo. A posição do elétron $i$ do átomo $n$, pode ser escrita em termos da posição do núcleo $\vec{R}_{i}$, e da distância do núcleo ao elétron $\vec{\rho}_{i}^{n}$, conforme a expressão

$$
\vec{r}_{i}^{n} \equiv \vec{R}_{i}+\vec{\rho}_{i}^{n}
$$

observe a figura.

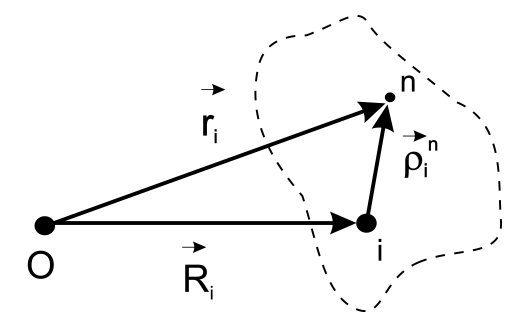

Figura 2.4: A posição da partícula, em termos da posição do núcleo e da posição dos elétrons.

A soma sobre as cargas pontuais pode ser reescrita segundo a forma

$$
\sum_{i=1}^{N} e^{i \vec{q} \cdot \vec{r}_{i}}=\sum_{\substack{a \text { atomos } \\ i=1}}^{N} e^{i \vec{q} \cdot \vec{R}_{i}} \sum_{n=1}^{P} e^{i \vec{q} \cdot \vec{\rho}_{i}^{n}}
$$

O termo referente à contribuição eletrônica pode ser tratado de forma independente da contribuição dos átomos. Portanto, é conveniente definir a contribuição eletrônica de um único átomo como

$$
F(q) \equiv \frac{1}{Z} \sum_{n=1}^{Z} e^{i \vec{q} \cdot \vec{\rho}_{i}^{n}}
$$

A razão das intensidades espalhada e incidente pode ser escrita como

$$
\frac{I(\theta, \omega)}{I_{0}}=\frac{|B(\theta, \omega)|^{2}}{R^{2}} F(q) F^{*}(q) \sum_{\substack{\text { atomos } \\ i, j=1}}^{N} e^{i \vec{q} \cdot\left(\vec{R}_{i}-\vec{R}_{j}\right)}
$$

O fator de forma se refere à distribuição espacial dos elétrons em um mesmo átomo, e é definido por

$$
P(q) \equiv|F(q)|^{2}
$$

O fator de estrutura é definido por

$$
S(q) \equiv \frac{1}{N}\left|\sum_{i=1}^{N} e^{i \vec{q} \cdot \vec{R}_{i}}\right|^{2}
$$

referente a interferência da radiação espalhada por diferentes átomos. 
A intensidade da luz espalhada pode ser escrita como um produto dos fatores de forma e estrutura,

$$
\frac{I(\theta, \omega)}{I_{0}}=\frac{|B(\theta, \omega)|^{2}}{R^{2}} P(q) N S(q) .
$$

De forma análoga podemos estender o conceito de fator de forma e fator de estrutura para espalhamento de luz de macromoléculas em solução, ver por exemplo (Johnson e Gabriel, 1994; Hiemenz, 1984). O fator de estrutura corresponde a uma soma sobre as posições dos centros de massa das macromoléculas, e o fator de forma refere-se a uma soma sobre a distribuição eletrônica de uma macromolécula.

As macromoléculas em solução não tem posições nem orientações de equilíbrio definidas, de forma que para obter a interferência da radiação espalhada é necessário ainda tomar uma média sobre o espaço de configurações, ou seja

$$
\frac{I(\theta, \omega)}{I_{0}}=\frac{|B(\theta, \omega)|^{2}}{R^{2}}\langle P(q)\rangle N\langle S(q)\rangle,
$$

onde a primeira média, sobre $P(q)$, corresponde a uma média sobre as todas as orientações da partícula (no ângulo sólido), e a média sobre $S(q)$ corresponde a uma média sobre as posições dos centros de massa.

\subsection{Fator de Forma}

Dada a equação

$$
\left.\langle P(q)\rangle\right|_{\text {rotação }}=\left.\frac{1}{Z^{2}}\left\langle\sum_{i} \sum_{j} \exp \left(i \vec{q} \cdot \vec{\rho}_{i j}\right)\right\rangle\right|_{\text {rotação }},
$$

a média sobre as orientações livres de uma dada forma fixa(flutuações térmicas de forma não estão sendo consideradas) é obtida por meio de uma integração sobre o ângulo sólido

$$
\begin{aligned}
\left.\left\langle\exp \left(i \vec{q} \cdot \vec{\rho}_{i j}\right)\right\rangle\right|_{\text {rotação }} & =\frac{\int \exp \left(i \vec{q} \cdot \vec{\rho}_{i j}\right) d \Omega}{\int d \Omega} \\
\left.\left\langle\exp \left(i q \rho_{i j} \cos \alpha\right)\right\rangle\right|_{\text {rotação }} & =\frac{\int_{0}^{2 \pi} \int_{0}^{\pi} \exp \left(i q \rho_{i j} \cos \alpha\right) \operatorname{sen} \alpha d \alpha d \phi}{\int_{0}^{2 \pi} \int_{0}^{\pi} \operatorname{sen} \alpha d \alpha d \phi} \\
& =\frac{1}{2} \int_{0}^{\pi} \exp \left(i q \rho_{i j} \cos \alpha\right) \operatorname{sen} \alpha d \alpha .
\end{aligned}
$$

Temos portanto

$$
\left.\langle P(q)\rangle\right|_{\text {rotação }}=\frac{1}{Z^{2}} \sum_{i} \sum_{j} \frac{\operatorname{sen}\left(q \rho_{i j}\right)}{q \rho_{i j}} .
$$




\subsubsection{Fator de forma para modelos esféricos}

O fator de forma de uma esfera de raio $R_{0}$ e com distribuição eletrônica uniforme pode ser calculado por meio de coordenadas esféricas,

$$
\begin{aligned}
F(q)_{\text {esfera }} & =\int_{0}^{2 \pi} \int_{0}^{\pi} \int_{0}^{R_{0}} \exp (i \vec{q} \cdot \vec{\rho}) \rho^{2} \operatorname{sen} \alpha d \rho d \alpha d \phi \\
& =2 \pi \int_{0}^{R_{0}} \rho^{2} d \rho \int_{0}^{\pi} \operatorname{sen} \alpha \exp (i q \rho \cos \alpha) d \alpha \\
& =\frac{4 \pi}{q} \int_{0}^{R_{0}} \rho \operatorname{sen}(q \rho) d \rho \\
& =\frac{4 \pi}{q^{3}}\left\{\operatorname{sen}\left(q R_{0}\right)-q R_{0} \cos \left(q R_{0}\right)\right\}
\end{aligned}
$$

portanto,

$$
P(q)_{\text {esfera }}=\left(\frac{4 \pi}{q^{3}}\right)^{2}\left\{\operatorname{sen}\left(q R_{0}\right)-q R_{0} \cos \left(q R_{0}\right)\right\}^{2} .
$$

Na figura 2.5 podemos ilustrar o fator de forma calculado para esferas de diferentes tamanhos.

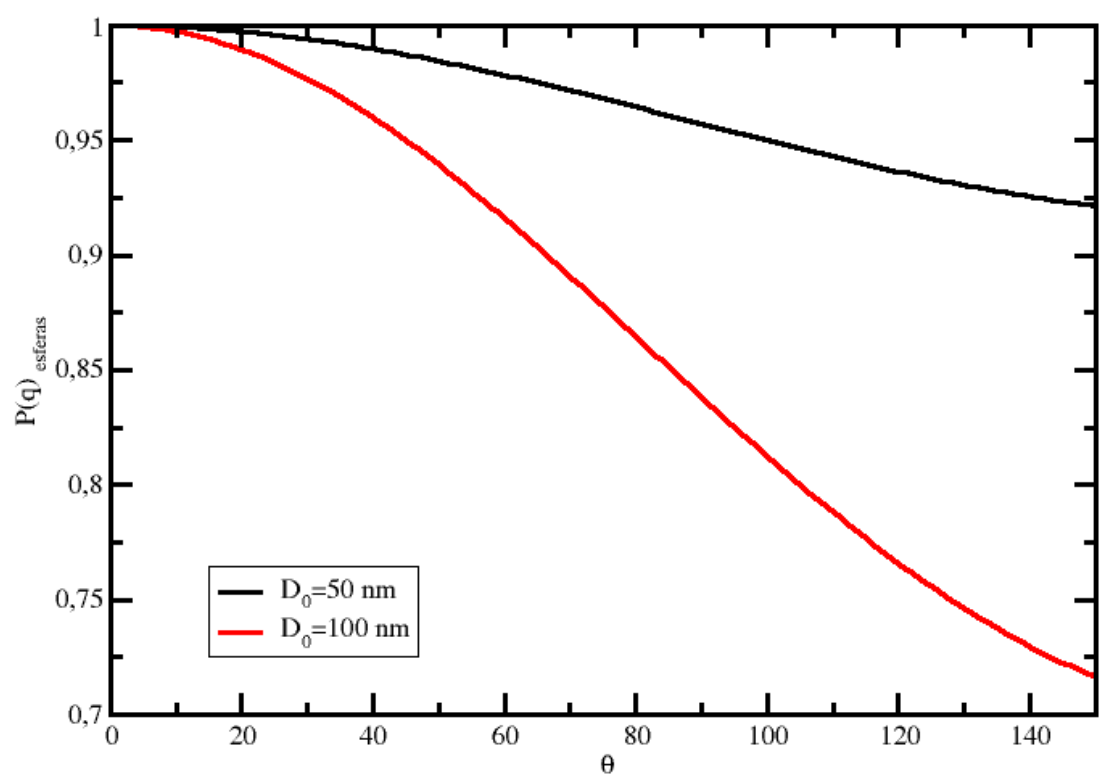

Figura 2.5: Fator de forma da esfera, $\lambda=633 \mathrm{~nm}$. 
De modo análogo, podemos calcular o fator de forma para uma casca esférica, de raio externo $R_{0}$ e espessura $r$, admitindo que a distribuição eletrônica é uniforme. Este, por sua vez, pode ser utilizado como modelo para bicamadas lipídicas (vesículas), onde $r$ representa a espessura da bicamada, observe a figura 2.6.

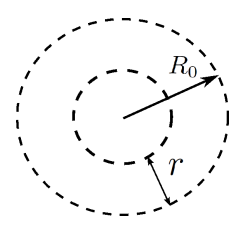

Figura 2.6: Casca esférica representando uma vesícula, $\lambda=633 \mathrm{~nm}$.

Das equações (2.24), temos

$$
\begin{aligned}
& F(q)_{\text {vesicula }}=\frac{4 \pi}{q} \int_{R_{0}-r}^{R_{0}} \rho d \rho \operatorname{sen} q \rho \\
= & \frac{4 \pi}{q^{3}}\left\{\operatorname{sen}\left(q R_{0}\right)-\operatorname{sen}\left(q\left(R_{0}-r\right)\right)-q R_{0} \cos \left(q R_{0}\right)+\left(q\left(R_{0}-r\right)\right) \cos \left(q\left(R_{0}-r\right)\right)\right\},
\end{aligned}
$$

portanto,

$$
P(q)_{\text {vesiculas }}=\left|F(q)_{\text {vesicula }}\right|^{2}
$$

Na figura 2.7 podemos ver alguns exemplos do fator de forma para vesículas.

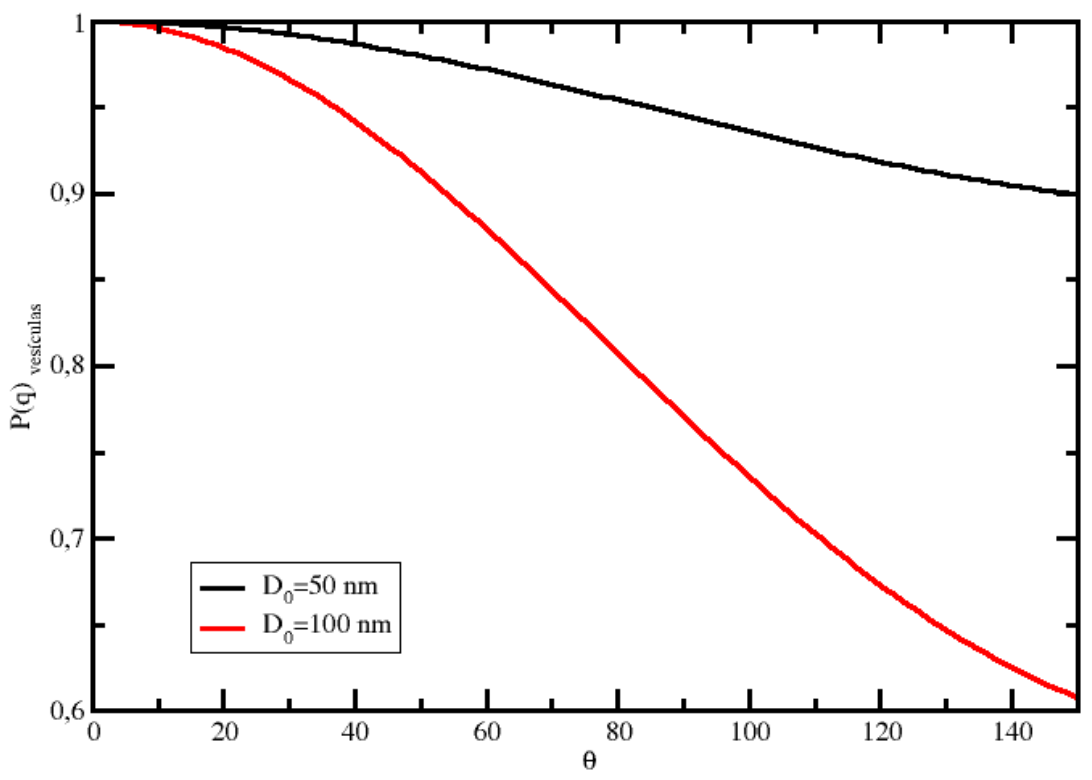

Figura 2.7: Fator de forma da casca esférica (vesícula), $\lambda=633 \mathrm{~nm}$. 
O fator de forma para vesículas possui um decaimento mais rápido do que o fator de forma para esferas, podemos comparar os fatores, com relação aos parâmetros $q D_{0}$.

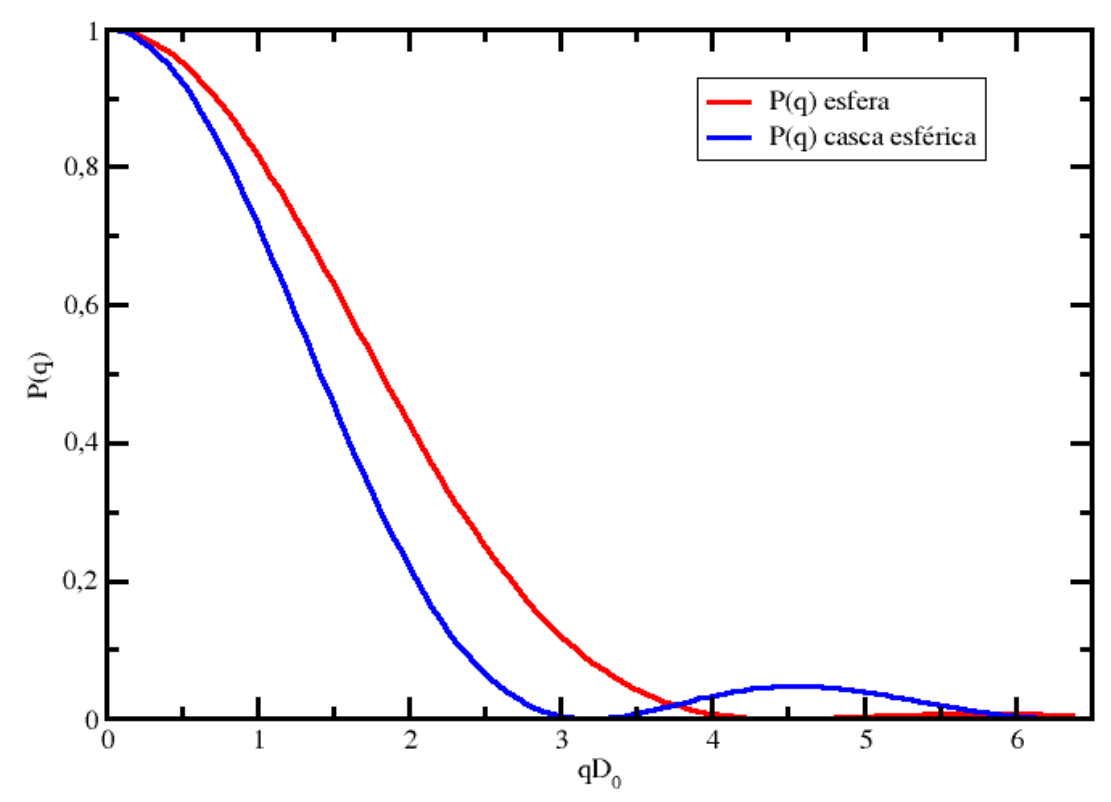

Figura 2.8: Comparação dos fatores de forma da esfera e da casca esférica, $\lambda=633 \mathrm{~nm}$.

\subsection{Fator de Estrutura}

O fator de estrutura está diretamente relacionado à função de distribuição radial, $g(r)$, que por sua vez está relacionada à probabilidade de encontrar um par de partículas a uma dada distância $r$ entre si. Podemos reescrever o fator de estrutura dado pela equação (2.19) separando a contribuição das partículas idênticas da dos pares de partículas distintas,

$$
\begin{aligned}
S(q) & =\frac{1}{N}\left\{N+\frac{1}{N} \sum_{i \neq j}\left\langle e^{i \vec{q} \cdot\left(\vec{R}_{i}-\vec{R}_{j}\right)}\right\rangle\right\} \\
& =\frac{1}{N}\left\{N+\frac{1}{N} \sum_{i \neq j}\left\langle\int d \vec{r} \delta\left(\vec{R}_{i}-\vec{R}_{j}-\vec{r}\right) e^{i \vec{q} \cdot \vec{r}}\right\rangle\right\} \\
& =1+\int d \vec{r} e^{i \vec{q} \cdot \vec{r}}\left\langle\frac{1}{N^{2}} \sum_{i \neq j} \delta\left(\vec{R}_{i}-\vec{R}_{j}-\vec{r}\right)\right\rangle
\end{aligned}
$$


A função de distribuição radial $g(r)$ é definida por

$$
g(\vec{r})=\frac{V}{N(N-1)}\left\langle\sum_{i \neq j}^{N} \delta\left(\vec{R}_{j}-\vec{R}_{i}-\vec{r}\right)\right\rangle,
$$

de forma que, para $N>>1$,

$$
g(\vec{r})=\frac{V}{N^{2}}\left\langle\sum_{i \neq j}^{N} \delta\left(\vec{R}_{j}-\vec{R}_{i}-\vec{r}\right)\right\rangle .
$$

Portanto, substituindo a equação (2.29) no fator de estrutura obtido pela equação (2.28), temos

$$
S(q)=1+\rho \int d \vec{r} \cdot g(\vec{r}) \exp (i \vec{q} \cdot \vec{r})
$$

Podemos ainda reescrever o fator de estrutura descrito na expressão anterior utilizando a definição da função delta, tal que

$$
\begin{aligned}
& S(q)=1+\rho \int d \vec{r} \cdot e^{i \vec{q} \cdot \vec{r}}[g(\vec{r})-1]+\rho \int d \vec{r} \cdot e^{i \vec{q} \cdot \vec{r}} \\
& S(q)=1+\rho(2 \pi)^{3} \delta(\vec{q}=0)+\rho \int_{-\infty}^{\infty} d \vec{r} \cdot e^{i \vec{q} \cdot \vec{r}}[g(\vec{r})-1] .
\end{aligned}
$$

onde $\rho(2 \pi)^{3} \delta(\vec{q}=0)$ corresponde à contribuição do feixe direto.

No regime muito diluído, como é o caso do gás ideal, por exemplo, a probabilidade de encontrar quaisquer pares de partículas a uma dada distância $r$ é a mesma, de forma que $g(r)=1$, e portanto seu fator de estrutura $S(q) \rightarrow 1$.

Podemos observar alguns exemplos do fator de estrutura, obtidos numericamente através da transformada de Fourier da $g(r)$, figura 2.9. Utilizamos uma $g(r)$ dada pela aproximação de Percus-Yevic, PY, (Smith e Henderson, 1970).

A aproximação de Percus-Yevic descreve apenas interações de volume excluído e $\rho^{*}$, representa a fração de volume ocupado, portanto quanto maiores as interações repulsivas impostas pelo volume excluído, maior a estrutura no líquido.

\subsection{Polarizabilidade}

Quando tratamos de cargas isoladas, a presença do campo elétrico imprime ao elétron ligado um movimento harmônico forçado, como vimos anteriormente. A presença da matéria pode causar modificações com relação a liberdade do elétron. Certos materiais exibem a propriedade que seus elétrons não tem liberdade para se moverem na presença de um campo elétrico aplicado. Contudo esse materiais, chamados de dielétricos, podem ser polarizados por um campo elétrico externo, (Fenkel, 2005). 


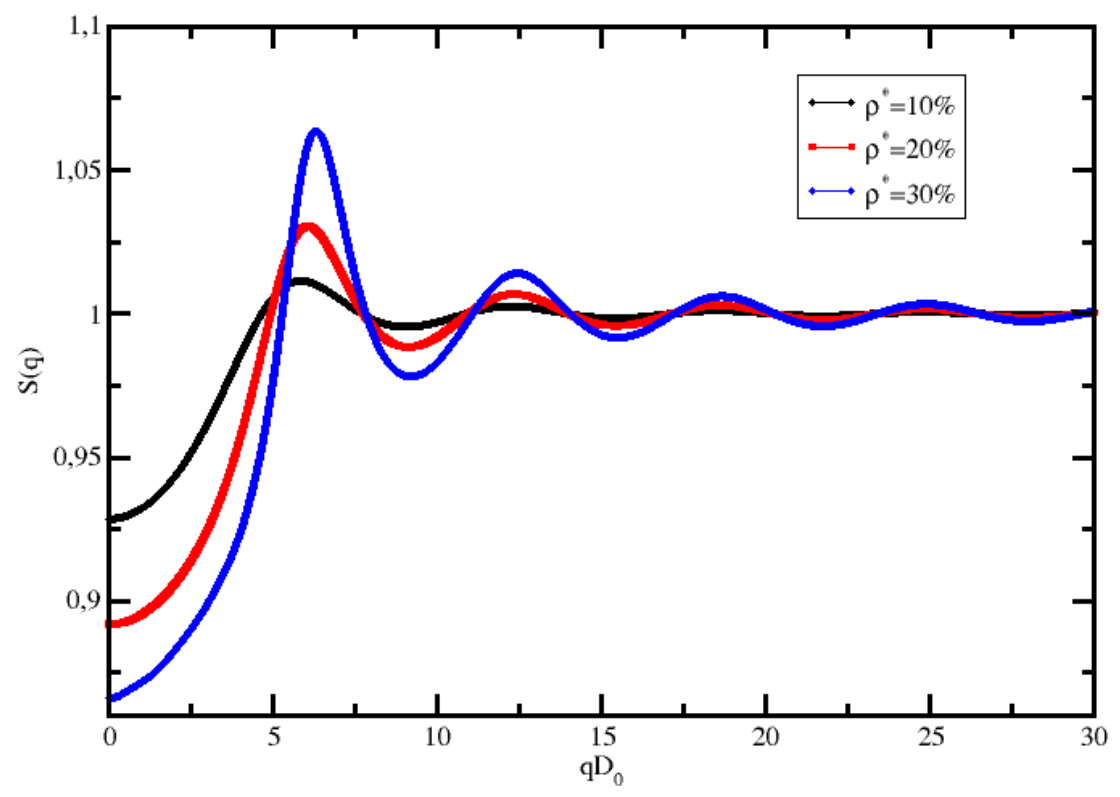

Figura 2.9: Fator de estrutura obtido a partir da transformada de Fourier da $g(r)$ dada pela aproximação de PY, para diferentes frações de volume ocupado.

Para tratarmos de moléculas precisamos introduzir o conceito de polarizabilidade molecular. A polarizabilidade molecular está relacionada com o momento de dipolo que a molécula assume na presença de um campo elétrico aplicado. Portanto, podemos escrever a relação

$$
\vec{\mu}_{i}=\alpha_{i} \epsilon_{0} \vec{E}
$$

onde $\alpha$ é o tensor polarizabilidade molecular, e a permissividade $\epsilon_{0}$ dimensiona a equação anterior no sistema internacional de unidades. As componentes do tensor polarizabilidade podem ser associadas aos eixos de simetria da molécula, (Williams, 1962). Para uma molécula isotrópica o tensor polarizabilidade pode ser tratado como uma grandeza escalar

$$
\alpha_{i}=\frac{1}{3}\left(\alpha_{x x}+\alpha_{y y}+\alpha_{z z}\right)
$$

onde $\alpha_{x x}=\alpha_{y y}=\alpha_{z z}$.

Para moléculas anisotrópicas, podemos calcular o momento de dipolo da molécula $i$, 
segundo a relação, (Williams, 1962):

$$
\begin{aligned}
& \mu_{x}=\epsilon_{0}\left(\alpha_{x x} E_{x}+\alpha_{x y} E_{y}+\alpha_{x z} E_{z}\right) \\
& \mu_{y}=\epsilon_{0}\left(\alpha_{y x} E_{x}+\alpha_{y y} E_{y}+\alpha_{y z} E_{z}\right) \\
& \mu_{z}=\epsilon_{0}\left(\alpha_{z x} E_{x}+\alpha_{z y} E_{y}+\alpha_{z z} E_{z}\right) .
\end{aligned}
$$

O momento de dipolo é dado por

$$
\vec{\mu}=-e \vec{r}
$$

onde $e$ corresponde a carga elementar do elétron.

Segundo o modelo de uma molécula polarizável na presença de um campo elétrico, haverá uma deformação da nuvem eletrônica, representada pelo deslocamento de cargas, conforme ilustrado na figura 2.10 .

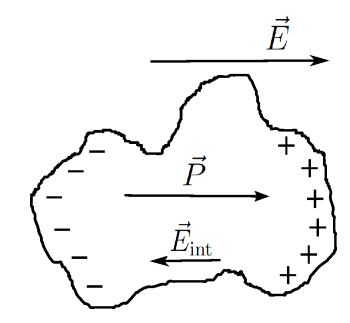

Figura 2.10: Ilustração da polarizabilidade e do campo interno molecular.

Através das equações (2.33) e (2.36) podemos escrever

$$
\epsilon_{0} \alpha \vec{E}=-e \vec{r}
$$

Para a incidência de uma onda plana, o campo elétrico pode ser descrito por

$$
\vec{E}=\vec{E}_{0} e^{i(\vec{k} \cdot \vec{r}-\omega t)}
$$

Dessa forma, com o uso das equações (2.37), (2.38), (2.3) temos

$$
\epsilon_{0} \alpha=\frac{e^{2}}{m} \frac{1}{\omega^{2}-\omega_{0}^{2}+i \Gamma \omega} .
$$

Portanto o campo elétrico da onda espalhada descrito pode ser reescrito como na equação $(2.39)$,

$$
\begin{gathered}
E(\theta, \omega, t)=\frac{\Pi(\theta)}{4 \pi \epsilon_{0} c^{2}} \underbrace{\frac{e^{2}}{m} \frac{\omega^{2}}{\omega^{2}-\omega_{0}^{2}+i \Gamma \omega}}_{\epsilon_{0} \alpha} \frac{E_{0} e^{i(\vec{k} \cdot \vec{r}-\omega t)}}{R} . \\
E(\theta, \omega, t)=\frac{\Pi(\theta)}{4 \pi c^{2}} \alpha \omega^{2} \frac{E_{0} e^{i(\vec{k} \cdot \vec{r}-\omega t)}}{R}
\end{gathered}
$$


onde

$$
\omega^{2}=\frac{4 \pi^{2} c^{2}}{\lambda^{2}}
$$

$\log \mathrm{O}$

$$
E(\theta, \omega, t)=\Pi(\theta) \frac{\pi}{\lambda^{2}} \alpha \frac{E_{0} e^{i(\vec{k} \cdot \vec{r}-\omega t)}}{R} .
$$

Portanto conforme vimos na equação (2.21), podemos escrever

$$
\frac{I(\theta, \omega)}{I_{0}}=\frac{\pi^{2} \Pi^{2}(\theta)}{\lambda^{4} R^{2}} \alpha^{2}\langle P(q)\rangle N\langle S(q)\rangle .
$$

\subsection{Misturas}

Vamos considerar agora uma solução com dois tipos de moléculas, $A$ e $B$. A informação sobre o sistema espalhador pode ser extraída de três fatores: a polarizabilidade molecular $\alpha$, a forma da distribuição eletrônica nas moléculas $F(q)$, e a interferência devida a luz espalhada por várias partículas, obtida do termo $\sum_{i=1}^{N} e^{i \vec{q} \cdot \vec{R}_{i}}$.

A partir da equação (2.40), o campo elétrico espalhado por uma solução é dado por

$$
\begin{aligned}
E(\theta, \omega, t) & =\Pi(\theta) \frac{\pi}{\lambda^{2}} \frac{E_{0} e^{\left(i \overrightarrow{k^{\prime}} \cdot \vec{R}\right)} e^{(-i \omega t)}}{R} \\
& \times\left\{\alpha_{A} F(q)_{A} \sum_{i=1}^{N_{A}} e^{\left(i \vec{q} \cdot \vec{R}_{A}\right)}+\alpha_{B} F(q)_{B} \sum_{i=1}^{N_{B}} e^{\left(i \vec{q} \cdot \vec{R}_{B}\right)}\right\} .
\end{aligned}
$$

Uma vez que a intensidade depende do quadrado do campo elétrico, a intensidade da luz espalhada apresenta um termo de mistura, como se observa nos dois últimos termos da equação (2.43),

$$
\begin{aligned}
\frac{I(\theta, \omega)}{I_{0}} & =\frac{\pi^{2} \Pi^{2}(\theta)}{\lambda^{4} R^{2}}\left\{\alpha_{A}^{2} P_{A}(q) \sum_{i, j=1}^{N_{A}} e^{i \vec{q}\left(\vec{R}_{i}^{A}-\vec{R}_{j}^{A}\right)}\right. \\
& +\alpha_{B}^{2} P_{B}(q) \sum_{i, j=1}^{N_{B}} e^{i \vec{q}\left(\vec{R}_{i}^{B}-\vec{R}_{j}^{B}\right)} \\
& +\alpha_{A} \alpha_{B}\left|F_{A}(q) F_{B}^{*}(q)\right| \sum_{i=1}^{N_{A}} \sum_{j=1}^{N_{B}} e^{i \vec{q}\left(\vec{R}_{i}^{A}-\vec{R}_{j}^{B}\right)} \\
& \left.+\alpha_{B} \alpha_{A}\left|F_{B}(q) F_{A}^{*}(q)\right| \sum_{i=1}^{N_{B}} \sum_{j=1}^{N_{A}} e^{i \vec{q}\left(\vec{R}_{i}^{B}-\vec{R}_{j}^{A}\right)}\right\} .
\end{aligned}
$$

A equação (2.43) pode ser reescrita tendo em vista as definições do fator de forma $P(q)$, e do fator de estrutura $S(q)$. Trataremos adiante os fatores de estrutura das espécies $A$ e $B$ 
e do termo de mistura, uma vez que o fator de forma pode ser identificado imediatamente com $\left|F_{\alpha}(q) F_{\beta}^{*}(q)\right|$, onde $\alpha, \beta=A, B$.

O fator de estrutura é definido pela equação (2.19). Podemos proceder de maneira análoga à apresentada na Seção 2.6, e escrever

$$
S_{\alpha, \alpha}(q)=1+\rho_{\alpha} \int d \vec{r} g(\vec{r}) e^{i \vec{q} \cdot \vec{r}},
$$

que equivale à equação (2.30), fazendo contato com a função de distribuição radial $g(r)$. Vamos analisar inicialmente a função de distribuição radial da mistura. O termo $N_{\alpha}\left(N_{\alpha}-\right.$ 1) da $g(r)$ se refere ao número de pares de cada espécie. Para uma mistura, porém, o número de pares formados com espécies dos tipos $A$ e $B$ é dado por $N_{A} N_{B}$. Assim, a $g(r)$ para a mistura das espécies $A$ e $B$ pode ser escrita na forma

$$
g(\vec{r})=\frac{V}{N_{A} N_{B}}\left\langle\sum_{i}^{N_{A}} \sum_{j}^{N_{B}} \delta\left(\overrightarrow{R_{j}^{A}}-\overrightarrow{R_{i}^{B}}-\vec{r}\right)\right\rangle .
$$

Podemos então generalizar a função de distribuição radial para descrever a relação entre pares de espécies iguais ou distintas,

$$
g_{\alpha, \beta}(\vec{r})=\frac{V}{N_{\alpha} N_{\beta}}\left\langle\sum_{i}^{N_{\alpha}} \sum_{j}^{N_{\beta}} \delta\left(\overrightarrow{R_{j}^{\alpha}}-\overrightarrow{R_{i}^{\beta}}-\vec{r}\right)\right\rangle,
$$

onde $\alpha, \beta=A, B$. Note que quando $\alpha=\beta$ retomamos a equação (2.29) para $N_{\alpha}>>1$.

Para estudar o fator de estrutura da mistura, é conveniente definir

$$
\begin{aligned}
& S_{A, B}(q)=\frac{1}{N_{A}}\left\langle\sum_{i=1}^{N_{A}} \sum_{j=1}^{N_{B}} e^{i \vec{q} \cdot\left(\vec{R}_{i}^{A}-\vec{R}_{j}^{B}\right)}\right\rangle, \\
& S_{B, A}(q)=\frac{1}{N_{B}}\left\langle\sum_{i=1}^{N_{B}} \sum_{j=1}^{N_{A}} e^{i \vec{q} \cdot\left(\vec{R}_{i}^{B}-\vec{R}_{j}^{A}\right)}\right\rangle .
\end{aligned}
$$

Observamos que o fator de estrutura para a mistura não possui os termos onde $i=j$, visto que a soma é sempre entre pares formados por espécies distintas. As equações (2.47) e (2.48) podem ser reescritas na forma

$$
\begin{aligned}
& S_{A, B}(q)=\frac{1}{N_{A}} \sum_{i}^{N_{A}} \sum_{j}^{N_{B}}\left\langle\int d \vec{r} \delta\left(\overrightarrow{R_{j}^{A}}-\overrightarrow{R_{i}^{B}}-\vec{r}\right) e^{i \vec{q} \cdot \vec{r}}\right\rangle, \\
& S_{B, A}(q)=\frac{1}{N_{B}} \sum_{i}^{N_{B}} \sum_{j}^{N_{A}}\left\langle\int d \vec{r} \delta\left(\overrightarrow{R_{j}^{B}}-\overrightarrow{R_{i}^{A}}-\vec{r}\right) e^{i \vec{q} \cdot \vec{r}}\right\rangle .
\end{aligned}
$$

Podemos então substituir a função de distribuição radial da mistura dada por (2.45) nas 
equações acima, de forma que

$$
\begin{aligned}
& S_{A, B}(q)=\frac{1}{N_{A}} \frac{N_{A} N_{B}}{V} \int d \vec{r} g_{A, B}(\vec{r}) e^{i \vec{q} \cdot \vec{r}}, \\
& S_{B, A}(q)=\frac{1}{N_{B}} \frac{N_{A} N_{B}}{V} \int d \vec{r} g_{B, A}(\vec{r}) e^{i \vec{q} \cdot \vec{r}} .
\end{aligned}
$$

Assim,

$$
\begin{aligned}
& S_{A, B}(q)=\rho_{B} \int d \vec{r} g_{A, B}(\vec{r}) e^{i \vec{q} \cdot \vec{r}} \\
& S_{B, A}(q)=\rho_{A} \int d \vec{r} g_{B, A}(\vec{r}) e^{i \vec{q} \cdot \vec{r}}
\end{aligned}
$$

onde introduzimos as densidades de partículas do tipo $A$ e $B\left(\rho_{A}\right.$ e $\left.\rho_{B}\right)$. Uma expressão geral para o fator de estrutura é dada por

$$
S_{\alpha, \beta}(q)=\delta_{\alpha, \beta}+\rho_{\beta} \int d \vec{r} g_{\alpha, \beta}(\vec{r}) e^{i \vec{q} \cdot \vec{r}}
$$

onde $\alpha, \beta=A, B$. Quando $\alpha=\beta$, temos $\delta_{\alpha, \beta}=1$, o que reproduz o fator de estrutura para cada espécie isoladamente, de acordo com a equação (2.44). Entretanto, quando $\alpha \neq \beta$, temos $\delta_{\alpha, \beta}=0$, reproduzindo o fator de estrutura para a mistura, definido segundo as equações $(2.50)$.

O termo $\delta_{\alpha, \beta}=1$ representa os termos da somatória, cujos índices $i$ e $j$ são iguais, $i=j$. Esses termos aparecem apenas quando olhamos para o fator de estrutura de uma única espécie, $A$ ou $B$. Já no caso da mistura, o fator de estrutura é analisado de uma espécie com relação a outra, deste modo os termos envolvidos na soma sempre possuem índices $i$ e $j$ diferentes, $i \neq j$.

Note que os fatores de estrutura da mistura $S_{A, B}(q)$ e $S_{B, A}(q)$ serão iguais apenas quando $\rho_{A}=\rho_{B}$. No entanto, podemos definir

$$
s_{\alpha, \beta}=\frac{S_{\alpha, \beta}}{\rho_{\beta}}=\int d \vec{r} g_{\alpha, \beta}(\vec{r}) e^{i \vec{q} \cdot \vec{r}}
$$

tal que $s_{A, B}=s_{B, A}$.

A intensidade da luz espalhada pode ser reescrita na forma

$$
\frac{I(\theta, \omega)}{I_{0}}=\frac{\pi^{2} \Pi^{2}(\theta)}{\lambda^{4} R^{2}}\left\{\sum_{\alpha}^{N_{\alpha}} \sum_{\beta}^{N_{\beta}} \alpha_{\alpha} \alpha_{\beta}\left|F_{\alpha}(q) F_{\beta}^{*}(q)\right| N_{\alpha} S_{\alpha, \beta}(q)\right\}
$$

onde $\alpha, \beta=A, B$, e $S_{\alpha, \beta}(q)$ é dado pela equação $(2.51)$. 


\subsection{Soluções}

Uma mistura de duas espécies, $A$ e $B$, é chamada uma solução quando a concentração de uma é muito maior do que a da outra. Rotularemos por $A$ o solvente e por $B$ o soluto da solução. É possível verificar que o fator de estrutura é independente do ângulo de espalhamento, podendo ser escrito em termos de flutuações de densidade, quando a distância média entre as partículas é muito menor que o comprimento da luz visível. De forma análoga ao que foi feito na equação (2.32), podemos separar a contribuição do fator de estrutura do feixe direto,

$$
\begin{aligned}
& S_{\alpha, \beta}(q)=\delta_{\alpha, \beta}+\rho_{\beta} \int d \vec{r} e^{i \vec{q} \cdot r}+\rho_{\beta} \int d \vec{r}\left[g_{\alpha, \beta}(\vec{r})-1\right] e^{i \vec{q} \cdot r} \\
& S_{\alpha, \beta}(q)=\delta_{\alpha, \beta}+4 \pi \rho_{\beta} \delta(q=0)+\rho_{\beta} \int d \vec{r}\left[g_{\alpha, \beta}(\vec{r})-1\right] e^{i \vec{q} \cdot r} .
\end{aligned}
$$

Não estamos interessados na contribuição do feixe direto $q=0$, deste modo

$$
S_{\alpha, \beta}(q \neq 0)=\delta_{\alpha, \beta}+\rho_{\beta} \int d \vec{r}[g(\vec{r})-1] e^{i \vec{q} \cdot r} .
$$

Vamos introduzir uma aproximação para escrever o fator de estrutura em termos das flutuações dos números de partículas,

$$
|\vec{q} \cdot \vec{r}|=4 \pi \operatorname{sen}\left(\frac{\theta}{2}\right) \frac{r}{\lambda} \cos (\theta)<<1,
$$

$\log \mathrm{O}$

$$
e^{(i \vec{q} \cdot \vec{r})} \approx 1
$$

essa aproximação é valida quando essa condição for satisfeita, $r<<\lambda$, para líquidos simples por exemplo, ou quando tomamos o limite de $q \rightarrow 0$.

Na equação de Zimm são considerados dois importantes limites, o limite da diluição infinita, onde a concentração de soluto tende a $C_{B} \rightarrow 0$, e o limite de ângulo nulo $q \rightarrow 0$, portanto essa aproximação sempre será válida na equação de Zimm. Nesta aproximação o fator de estrutura independe do ângulo de espalhamento.

A aproximação acima para todas as espécies da solução implica

$$
S_{\alpha, \beta}=\delta_{\alpha, \beta}+\rho_{\beta} \int d \vec{r}[g(\vec{r})-1] .
$$

Veremos adiante como a equação (2.55) se relaciona a flutuações na densidade. A densidade local pode ser escrita como

$$
\rho_{\alpha}(\vec{r})=\sum_{i}^{N_{\alpha}} \delta\left(\vec{r}_{i}^{\alpha}-\vec{r}\right) .
$$


Além disso, o número de partículas em um determinado volume é

$$
N_{\alpha}=\int d \vec{r} \rho_{\alpha}(\vec{r}) .
$$

Assim,

$$
\begin{aligned}
N_{\alpha} N_{\beta} & =\int d \vec{r}^{\prime} \rho_{\alpha}\left(\vec{r}^{\prime}\right) \int d \vec{r}^{\prime \prime} \rho_{\beta}\left(\vec{r}^{\prime \prime}\right) \\
& =\sum_{i}^{N_{\alpha}} \int d \vec{r}^{\prime} \delta\left(\vec{r}_{i}^{\alpha}-\vec{r}^{\prime}\right) \sum_{j}^{N_{\beta}} \int d \vec{r}^{\prime \prime} \delta\left(\vec{r}_{j}^{\beta}-\vec{r}^{\prime \prime}\right),
\end{aligned}
$$

de forma que, tomando a média sobre as configurações

$$
\begin{aligned}
\left\langle N_{\alpha} N_{\beta}\right\rangle & =\int d \vec{r}^{\prime} \int d \vec{r}^{\prime \prime}\left\langle\sum_{i=j}^{N_{\alpha}} \delta\left(\vec{r}_{i}^{\alpha}-\vec{r}^{\prime}\right) \delta\left(\vec{r}_{j}^{\beta}-\vec{r}^{\prime \prime}\right)\right\rangle \delta_{\alpha, \beta}+ \\
& +\int d \vec{r}^{\prime} \int d \vec{r}^{\prime \prime}\left\langle\sum_{i}^{N_{\alpha}} \sum_{j}^{N_{\beta}} \delta\left(\vec{r}_{i}^{\alpha}-\vec{r}^{\prime}\right) \delta\left(\vec{r}_{j}^{\beta}-\vec{r}^{\prime \prime}\right)\right\rangle .
\end{aligned}
$$

Para $\alpha \neq \beta$ não existem os termos $i=j$. A partir da substituição $\vec{r}^{\prime \prime}=\vec{r}^{\prime}-\vec{r}$, obtemos

$$
\left\langle N_{\alpha} N_{\beta}\right\rangle=\left\langle N_{\alpha}\right\rangle \delta_{\alpha, \beta}+\left\langle\sum_{i}^{N_{\alpha}} \sum_{j}^{N_{\beta}} \int d \vec{r} \delta\left(\vec{r}_{j}^{\beta}-\vec{r}_{i}^{\alpha}-\vec{r}^{\prime}\right)\right\rangle,
$$

ou ainda,

$$
\left\langle N_{\alpha} N_{\beta}\right\rangle=\left\langle N_{\alpha}\right\rangle \delta_{\alpha, \beta}+\frac{\left\langle N_{\alpha}\right\rangle\left\langle N_{\beta}\right\rangle}{V} \int d \vec{r} g(\vec{r}) .
$$

onde utilizamos a definição da função de distribuição radial, obtida na equação (2.46) para uma mistura. Podemos dividir todos os termos de (2.58) por $V^{2}$ e subtrair $\left\langle\rho_{\alpha}\right\rangle\left\langle\rho_{\beta}\right\rangle$ de ambos os lados, de forma que

$$
\left\langle\rho_{\alpha} \rho_{\beta}\right\rangle-\left\langle\rho_{\alpha}\right\rangle\left\langle\rho_{\beta}\right\rangle=\frac{\left\langle\rho_{\alpha}\right\rangle}{V} \delta_{\alpha, \beta}+\frac{\left\langle\rho_{\alpha}\right\rangle\left\langle\rho_{\beta}\right\rangle}{V} \int d \vec{r} g(\vec{r})-\left\langle\rho_{\alpha}\right\rangle\left\langle\rho_{\beta}\right\rangle .
$$

A equação anterior pode ser rearranjada por meio da soma e subtração de 1 na integral, seguida por uma divisão por $\left\langle\rho_{\alpha}\right\rangle\left\langle\rho_{\beta}\right\rangle$,

$$
\begin{aligned}
\frac{\left\langle\rho_{\alpha} \rho_{\beta}\right\rangle-\left\langle\rho_{\alpha}\right\rangle\left\langle\rho_{\beta}\right\rangle}{\left\langle\rho_{\alpha}\right\rangle\left\langle\rho_{\beta}\right\rangle}=\frac{\left\langle\rho_{\alpha}\right\rangle}{V} \delta_{\alpha, \beta} & +\frac{\left\langle\rho_{\alpha}\right\rangle\left\langle\rho_{\beta}\right\rangle}{V} \int d \vec{r}[g(\vec{r})-1] \\
& +\frac{\left\langle\rho_{\alpha}\right\rangle\left\langle\rho_{\beta}\right\rangle}{V} \int d \vec{r}-\left\langle\rho_{\alpha}\right\rangle\left\langle\rho_{\beta}\right\rangle .
\end{aligned}
$$

Portanto,

$$
\frac{\left\langle\rho_{\alpha} \rho_{\beta}\right\rangle-\left\langle\rho_{\alpha}\right\rangle\left\langle\rho_{\beta}\right\rangle}{\left\langle\rho_{\alpha}\right\rangle\left\langle\rho_{\beta}\right\rangle}=\frac{1}{V \rho_{\beta}} \delta_{\alpha, \beta}+\frac{1}{V} \int d \vec{r}[g(\vec{r})-1]
$$


onde,

$$
\frac{\left\langle\rho_{\alpha} \rho_{\beta}\right\rangle-\left\langle\rho_{\alpha}\right\rangle\left\langle\rho_{\beta}\right\rangle}{\left\langle\rho_{\alpha}\right\rangle\left\langle\rho_{\beta}\right\rangle}=\frac{\left\langle N_{\alpha} N_{\beta}\right\rangle-\left\langle N_{\alpha}\right\rangle\left\langle N_{\beta}\right\rangle}{\left\langle N_{\alpha}\right\rangle\left\langle N_{\beta}\right\rangle} .
$$

Definimos, portanto, a flutuação no número de partículas como

$$
\sigma_{\alpha, \beta} \equiv\left\langle N_{\alpha} N_{\beta}\right\rangle-\left\langle N_{\alpha}\right\rangle\left\langle N_{\beta}\right\rangle
$$

As equações (2.60) e (2.62) resultam

$$
\int d \vec{r}[g(\vec{r})-1]=\frac{\sigma_{\alpha, \beta}}{N_{\alpha} N_{\beta}} V-\frac{\delta_{\alpha, \beta}}{\left\langle\rho_{\beta}\right\rangle} .
$$

O fator de estrutura pode então ser obtido da equação (2.54),

$$
S_{\alpha, \beta}=\delta_{\alpha, \beta}+\rho_{\beta} \frac{\sigma_{\alpha, \beta}}{N_{\alpha} N_{\beta}} V-\rho_{\beta} \frac{\delta_{\alpha, \beta}}{\rho_{\beta}}
$$

ou ainda

$$
S_{\alpha, \beta}=\frac{\sigma_{\alpha, \beta}}{N_{\alpha}}
$$

A equação (2.64) pode ser aplicada à expressão obtida em (2.53), de forma que a intensidade da luz espalhada seja escrita em termos das flutuações de densidade das espécies $A$ e $B$

$$
\frac{I(\theta, \omega)}{I_{0}}=\frac{\pi^{2} \Pi^{2}(\theta)}{\lambda^{4} R^{2}}\left\{\sum_{\alpha}^{N_{\alpha}} \sum_{\beta}^{N_{\beta}} \alpha_{\alpha} \alpha_{\beta}\left|F_{\alpha}(q) F_{\beta}^{*}(q)\right| \sigma_{\alpha, \beta}\right\}
$$

Para moléculas com distribuições eletrônicas simétricas, $\left|F_{\alpha}(q) F_{\beta}^{*}(q)\right|=\left|F_{\beta}(q) F_{\alpha}^{*}(q)\right|$, com $\alpha \neq \beta$, de forma que o fator de forma da mistura pode ser definido por

$$
P_{\alpha, \beta}(q)=\left|F_{\alpha}(q) F_{\beta}^{*}(q)\right|=\left|F_{\beta}(q) F_{\alpha}^{*}(q)\right|, \quad \alpha \neq \beta
$$

Como a flutuação de densidade para o termo de mistura não depende da permutação dos índices, $\sigma_{A, B}=\sigma_{B, A}$, podemos agrupar os termos referentes a mistura, e escrever simplesmente

$$
\begin{array}{r}
\frac{I(\theta, \omega)}{I_{0}}=\frac{\pi^{2} \Pi^{2}(\theta)}{\lambda^{4} R^{2}}\left\{\alpha_{A}^{2} P_{A, A} \sigma_{A, A}\right. \\
+\alpha_{B}^{2} P_{B, B} \sigma_{B, B}+ \\
\left.+2 \alpha_{A} \alpha_{B} P_{A, B} \sigma_{A, B}\right\}
\end{array}
$$

Deste modo, intensidade da luz espalhada depende da contribuição do solvente, do soluto e de um fator de mistura. 


\subsection{Relações termodinâmicas}

Podemos escrever a pressão total de uma solução como a soma da pressão do solvente puro $p_{0}(A)$ com a pressão adicional (osmótica), $\pi$, ocasionada pela adição do soluto (B). Desta forma, a relação termodinâmica de Gibbs-Duhem, ver por exemplo (Salinas, 2008; Oliveira, 2005) fica dada por

$$
S d T-V d\left(p_{0}+\pi\right)+\sum_{\alpha} N_{\alpha} d \mu_{\alpha}=0
$$

ou ainda, a temperatura constante,

$$
\begin{gathered}
V d\left(p_{0}+\pi\right)=\sum_{\alpha} N_{\alpha} d \mu_{\alpha} \Rightarrow \\
d\left(p_{0}+\pi\right)=\rho_{A} d \mu_{A}+\rho_{B} d \mu_{B} .
\end{gathered}
$$

No regime diluído que estamos considerando, a variação do potencial químico do solvente em relação ao número de partículas do soluto $N_{B}$ é desprezível, de forma que a equação (2.69) resulta

$$
\frac{d \pi}{d N_{B}}=\rho_{B} \frac{d \mu_{B}}{d N_{B}}
$$

Podemos associar a derivada do lado direito da equação (2.70) a uma flutuação no número de partículas. Para isso, lembramos que a probabilidade de um dado estado com $N_{A}$ partículas do tipo $A$ e $N_{B}$ partículas do tipo $B$ é dada no ensemble Grande-Canônico, ver por exemplo (Salinas, 2008)

$$
P=\frac{\exp \left(\beta\left(\mu_{A} N_{A}+\mu_{B} N_{B}\right)\right) \mathcal{Z}_{A B}}{\Xi_{A B}},
$$

onde $\mathcal{Z}_{A B} \equiv \mathcal{Z}\left(T, V, N_{A}, N_{B}\right)$, e a grande função de partição $\Xi_{A B}$ é dada por

$$
\Xi_{A B} \equiv \Xi\left(T, V, \mu_{A}, \mu_{B}\right)=\sum_{N_{A}} \sum_{N_{B}} \exp \left(\beta\left(\mu_{A} N_{A}+\mu_{B} N_{B}\right)\right) \mathcal{Z}\left(T, V, N_{A}, N_{B}\right) .
$$

Podemos então verificar que

$$
\frac{d\left\langle N_{\alpha}\right\rangle}{d \mu_{\beta}}=\beta\left\{\left\langle N_{\alpha} N_{\beta}\right\rangle-\left\langle N_{\alpha}\right\rangle\left\langle N_{\beta}\right\rangle\right\}=\beta \sigma_{\alpha, \beta},
$$

onde utilizamos a definição da flutuação do número de partículas dada pela equação (2.62). As equações (2.70) e (2.73) podem ser usadas para escrever a variação da pressão do soluto com respeito ao número de partículas na forma

$$
\frac{d \pi}{d N_{B}}=\rho_{B} \frac{1}{\beta \sigma_{B, B}}
$$


ou ainda, em termos da variação da densidade,

$$
\frac{d \pi}{d \rho_{B}}=\frac{d \pi}{d N_{B}} \frac{d N_{B}}{d \rho_{B}}=V \frac{d \pi}{d N_{B}},
$$

$\log \mathrm{O}$

$$
\frac{d \pi}{d \rho_{B}}=N_{B} \frac{1}{\beta \sigma_{B, B}} .
$$

A pressão do soluto pode ser escrita como uma expansão na densidade de partículas

$$
\pi=k_{B} T \rho_{B}\left\{1+a_{2} \rho_{B}+\cdots\right\}
$$

de forma que podemos escrever

$$
\frac{d \pi}{d \rho_{B}}=k_{B} T\left\{1+2 a_{2} \rho_{B}+\cdots\right\}
$$

onde $a_{2}$, que tem a dimensão do volume, é chamado "segundo coeficiente do Virial". Note que podemos desprezar termos de ordem maior ou igual a $\rho_{B}^{3}$, no regime diluído.

Conforme descrito pela equação 2.67 a intensidade da luz espalhada depende da contribuição do solvente, do soluto e de um fator de mistura. Entretanto, faremos algumas aproximações para simplificar a equação (2.67).

Primeiro, a intensidade da luz espalhada pela solução, em geral, é muito maior que a intensidade da luz espalhada pelo solvente. Quando isso não ocorre é necessário medir a intensidade da luz espalhada apenas pelo solvente, e subtrair a contribuição da solução. Muitos softwares comerciais para análise de Zimm permitem fazer essa correção. As dispersões lipídicas que trabalhamos, por exemplo, espalham ordens de grandeza mais que o solvente. Isso também pode depender da concentração em que está se trabalhando. Portanto como uma primeira aproximação vamos desprezar a contribuição do solvente, admitindo que essa é muito inferior que a da solução.

Além disso, da relação (2.73) temos que

$$
\beta \sigma_{A, B}=\frac{d N_{A}}{d \mu_{B}} .
$$

No regime diluído que estamos considerando, pequenas variações na concentração de soluto, B, provocaram variações muito pequenas no número de partículas do solvente, isto é,

$$
\frac{d N_{A}}{d \mu_{B}} \approx 0
$$

e portanto,

$$
\sigma_{A, B} \approx 0
$$

Então a intensidade da luz espalhada pode ser escrita apenas com a contribuição do soluto, segundo a relação 


$$
\frac{I(\theta, \omega)}{I_{0}}=\frac{\pi^{2} \Pi^{2}(\theta)}{\lambda^{4} R^{2}}\left\{\alpha_{B}^{2} P_{B, B} \sigma_{B, B}\right\} .
$$

Como descrito anteriormente o objetivo deste Capítulo consiste em obter a equação de Zimm. Para isso, precisamos de três importantes relações. A primeira que vamos descrever é a expansão no fator de estrutura, seguida da expansão no fator forma e finalizando com a relação de Claussius-Mossoti, com a relação de polarizabilidade e o incremento no índice de refração.

\subsection{Expansão do fator de estrutura}

Em física experimental é mais usual medir a concentração em massa, ao invés da densidade de partículas, de forma que se torna conveniente modificarmos a equação (2.77) tendo em vista as relações

$$
\begin{aligned}
& \rho_{B}=\frac{N_{B}}{V}, \quad N_{B}=n N_{A v}, \quad n=\frac{m}{M}, \\
& \rho_{B}=\frac{C_{B} N_{A v}}{M}, \\
& n R=N_{B} k_{B}, \quad k_{B}=\frac{R}{N_{A v}} .
\end{aligned}
$$

A variação da pressão osmótica com a concentração do soluto é dada por

$$
\frac{d \pi}{d C_{B}}=\frac{d \pi}{d \rho_{B}} \frac{d \rho_{B}}{d C_{B}}=\frac{N_{A v}}{M} \frac{d \pi}{d \rho_{B}},
$$

de forma que a equação (2.77) pode ser reescrita na forma

$$
\frac{d \pi}{d C_{B}}=\frac{R T}{M}\left\{1+2 a_{2} \frac{N_{A v}}{M} C_{B}+\cdots\right\} .
$$

Podemos escrever então uma expressão semelhante à usualmente encontrada na literatura de espalhamento de luz definindo

$$
A_{2}=\frac{a_{2} N_{A v}}{M^{2}}
$$

cuja unidade $\left[A_{2}\right]=\mathrm{cm}^{3} \mathrm{~mol} / \mathrm{g}^{2}$, de acordo com a bibliografia existente. Assim

$$
\frac{d \pi}{d C_{B}}=R T\left\{\frac{1}{M}+2 A_{2} C_{B}+\cdots\right\}
$$

conforme podemos encontrar em (Hiemenz, 1984).

De acordo com a equação (2.75) e as relações descritas acima, podemos escrever

$$
\frac{d \pi}{d C_{B}}=\frac{N_{A v}}{M} \frac{N_{B}}{\beta \sigma_{B, B}},
$$


de forma que a flutuação no número de partículas de soluto é dada por

$$
\sigma_{B, B}=\frac{N_{B}}{M}\left\{\frac{1}{M}+2 A_{2} C_{B}\right\}^{-1},
$$

ou se escrevemos em termos da concentração de soluto, temos

$$
\sigma_{B, B}=\frac{C_{B} N_{a v} V}{M}\left\{\frac{1}{M}+2 A_{2} C_{B}\right\}^{-1} .
$$

Substituindo a equação (2.84) na equação (2.78), temos

$$
\frac{I(\theta, \omega)}{I_{0}}=\frac{\pi^{2} \Pi^{2}(\theta)}{\lambda^{4} R^{2}}\left\{\alpha_{B}^{2} P_{B, B} \frac{C_{B} N_{a v} V}{M}\left\{\frac{1}{M}+2 A_{2} C_{B}\right\}^{-1}\right\}
$$

\subsection{Expansão do fator de forma}

Da expressão obtida para o fator de forma, equação 2.23 (Johnson e Gabriel, 1994),

$$
\langle P(q)\rangle=\frac{1}{Z^{2}} \sum_{i} \sum_{j} \frac{\operatorname{sen}\left(q \rho_{i j}\right)}{q \rho_{i j}} .
$$

Podemos expandir o $\operatorname{sen}\left(q \rho_{i j}\right)$,

$$
\operatorname{sen}\left(q \rho_{i j}\right)=\left(q \rho_{i j}\right)-\frac{\left(q \rho_{i j}\right)^{3}}{3 !}+\frac{\left(q \rho_{i j}\right)^{5}}{5 !}-\cdots
$$

para valores de $\left(q \rho_{i j}\right)$ pequenos, temos,

$$
\begin{aligned}
\langle P(q)\rangle & \approx \frac{1}{Z^{2}} \sum_{i} \sum_{j} 1-\frac{\left(q \rho_{i j}\right)^{2}}{3 !} \\
& \approx 1-\frac{1}{Z^{2}} \sum_{i} \sum_{j} \frac{\left(q \rho_{i j}\right)^{2}}{3 !}
\end{aligned}
$$

Uma das definições do raio de giração, (Johnson e Gabriel, 1994), é dada por

$$
R_{g}^{2}=\frac{1}{Z^{2}} \sum_{i} \sum_{j} \frac{\left(q \rho_{i j}\right)^{2}}{2}
$$

Portanto o fator de forma pode ser escrito sob a forma

$$
\langle P(q)\rangle=1-\frac{R_{g}^{2} q^{2}}{3} .
$$

Substituindo a equação 2.88 na equação 2.85 temos

$$
\frac{I(\theta, \omega)}{I_{0}}=\frac{\pi^{2} \Pi^{2}(\theta)}{\lambda^{4} R^{2}}\left\{\alpha_{B}^{2}\left\{1-\frac{R_{g}^{2} q^{2}}{3}\right\} \frac{C_{B} N_{a v} V}{M}\left\{\frac{1}{M}+2 A_{2} C_{B}\right\}^{-1}\right\}
$$

Podemos calcular o raio de giração para esferas e vesículas como fizemos anteriormente na seção 2.5.1. Se expandirmos as funções $\operatorname{sen}\left(q R_{0}\right)$ e $\cos \left(q R_{0}\right)$ das equações 2.25 para 
esferas e 2.27 para vesículas, e comparamos com a expressão anterior (2.88), facilmente chegaremos nas relações,

$$
R_{g}^{2}=\frac{3}{5} R_{0}^{2}
$$

para esferas, (Johnson e Gabriel, 1994) e

$$
R_{g}^{2}=\frac{3}{5} R_{0}^{2} \frac{1-\left(1-r / R_{0}\right)^{5}}{1-\left(1-r / R_{0}\right)^{3}},
$$

para vesículas, onde $R_{0}$ corresponde ao raio externo e $r$ a espessura da bicamada.

\subsection{Relação de Claussius Mossotti}

Dadas as equações de Maxwell, ver por exemplo (Fenkel, 2005)

$$
\begin{aligned}
& \vec{\nabla} \cdot \vec{E}=\frac{\rho}{\epsilon_{0}}, \quad \vec{\nabla} \cdot \vec{B}=0 \\
& \vec{\nabla} \times \vec{E}=-\frac{\partial \vec{B}}{\partial t}, \quad \vec{\nabla} \times \vec{B}=\mu_{0} \vec{J}+\mu_{0} \epsilon_{0} \frac{\partial \vec{E}}{\partial t}
\end{aligned}
$$

e a propriedade vetorial

$$
\vec{\nabla} \times(\vec{\nabla} \times \vec{E})=\vec{\nabla}(\vec{\nabla} \cdot \vec{E})-\vec{\nabla}^{2} E
$$

podemos escrever, haja vista $\vec{\nabla}(\vec{\nabla} \cdot \vec{E})=0$,

$$
\vec{\nabla} \times\left(-\frac{\partial \vec{B}}{\partial t}\right)=-\vec{\nabla}^{2} E .
$$

Invertendo a ordem de aplicação do rotacional e da derivada temporal de $\vec{B}$ (eles comutam), temos

$$
\vec{\nabla}^{2} E=\frac{\partial}{\partial t}\left\{\mu_{0} \vec{J}+\mu_{0} \epsilon_{0} \frac{\partial \vec{E}}{\partial t}\right\} .
$$

Podemos agora usar a equação da continuidade

$$
\vec{\nabla} \cdot \vec{J}+\frac{\partial \rho^{\prime}}{\partial t}=0
$$

onde $\rho^{\prime}$ corresponde à densidade de carga, que também pode ser escrita em termos do divergente da polarização,

$$
\rho^{\prime}=-\vec{\nabla} \cdot \vec{P}
$$

para escrever

$$
\vec{\nabla} \cdot \vec{J}=\frac{\partial \vec{\nabla} \cdot \vec{P}}{\partial t}
$$


portanto,

$$
\vec{J}=\frac{\partial \vec{P}}{\partial t}
$$

ou ainda, substituindo a equação (2.96) em (2.93),

$$
\vec{\nabla}^{2} E-\frac{1}{c^{2}} \frac{\partial^{2} \vec{E}}{\partial^{2} t}=\frac{1}{\epsilon c^{2}} \frac{\partial^{2} \vec{P}}{\partial^{2} t} .
$$

A polarização pode ser escrita como a soma dos momentos de dipolos por unidade de volume,

$$
\vec{P}=\frac{1}{V} \sum_{i}^{N} \vec{\mu}_{i}
$$

O momento de dipolo, definido anteriormente na equação (2.33), pode ser escrito na forma

$$
\vec{\mu}_{i}=\epsilon_{0} \alpha \vec{E}_{i}
$$

Além disso, o campo elétrico total depende do campo externo e do gerado pela polarizabilidade molecular

$$
\begin{array}{r}
\vec{E}_{i}=\vec{E}_{\text {ext }}+\vec{E}_{m o l} \Rightarrow \\
\vec{E}_{i}=\vec{E}_{\text {ext }}+\frac{\vec{P}}{3 \epsilon_{0}} .
\end{array}
$$

Assim, podemos escrever, considerando que todos os dipolos sejam iguais,

$$
\begin{gathered}
\vec{P}=\frac{N \vec{\mu}_{i}}{V}=\rho \epsilon_{0} \alpha \vec{E}_{i} \Rightarrow \\
\vec{P}=\rho \epsilon_{0} \alpha\left(\vec{E}_{\text {ext }}+\frac{\vec{P}}{3 \epsilon_{0}}\right),
\end{gathered}
$$

portanto

$$
\vec{P}=\frac{\rho \epsilon_{0} \alpha}{1-\rho \alpha / 3} \vec{E}_{e x t}
$$

As equações (2.100) e (2.97) resultam

$$
\vec{\nabla}^{2} E-\frac{1}{c^{2}} \frac{\partial^{2} \vec{E}}{\partial^{2} t}=\frac{1}{\epsilon c^{2}} \frac{\partial^{2}}{\partial^{2} t}\left(\frac{\rho \epsilon_{0} \alpha}{1-\rho \alpha / 3} \vec{E}_{e x t}\right),
$$

de forma que, considerando uma onda plana se propagando, por exemplo, no eixo x,

$$
\vec{E}_{e x t}=\vec{E}_{0} \exp (i(k x-\omega t))
$$

verificamos que

$$
k^{2}=\frac{\omega_{0}^{2}}{c^{2}} \frac{\rho \alpha}{1-\rho \alpha / 3}+\frac{\omega_{0}^{2}}{c^{2}} .
$$

Lembramos que

$$
n=\frac{c}{v} \quad v=\frac{\omega}{k} \quad n=\frac{k c}{\omega}
$$


de forma que obtemos a chamada "equação de Claussius-Mossotti",

$$
3\left(\frac{n^{2}-1}{n^{2}+2}\right)=\rho \alpha .
$$

Uma vez que o índice de refração da solução depende, em geral, da concentração do soluto, podemos escrever a expansão

$$
n=n_{0}+\frac{d n}{d C_{B}} C_{B}+\cdots
$$

onde $n_{0}$ representa o índice de refração na ausência do soluto, ou seja, o índice de refração do solvente. Para soluções diluídas, ordens superiores a $c$ podem ser desprezadas, de forma que

$$
n^{2}=n_{0}^{2}+2 n_{0} \frac{d n}{d C_{B}} C_{B}+\cdots
$$

Podemos substituir a relação acima na equação (2.103) de forma a obter, após algumas manipulações algébricas,

$$
3\left(\frac{n_{0}^{2}-1}{n_{0}^{2}+2}\right)+2 \frac{9}{\left(n_{0}^{2}+2\right)^{2}} n_{0} \frac{d n}{d C_{B}} C_{B}=\rho \alpha,
$$

Alguns textos da literatura (Johnson e Gabriel, 1994; Hiemenz, 1984) reduzem a equação (2.105), utilizando uma aproximação para gases, onde $n_{0} \approx 1$. Com essa aproximação é possível observar que o termo dominante dessa série tende a

$$
2 n_{0} \frac{d n}{d C_{B}} C_{B}=\rho \alpha,
$$

ou ainda, escrevendo a densidade de partículas do soluto em termos de sua concentração,

$$
2 n_{0} \frac{d n}{d C_{B}} \frac{M}{N_{A v}}=\alpha
$$

No entanto, para solventes questionamos se essa aproximação não seria um tanto grosseira, pois o índice de refração da água, por exemplo, corresponde a $n_{\text {agua }}=1,33$. Esta é uma questão que ainda ficou em aberto na apostila que vem sendo desenvolvida. Achamos importante ressaltar que para líquidos a aproximação utilizada inicialmente para gases, pode ser imprecisa.

Contudo, seguiremos o desenvolvimento com a aproximação encontrada nos livros, para obter a equação de Zimm que foi utilizada, nesse trabalho.

\subsection{A Equação de Zimm}

Substituindo a equação (2.107) na equação (2.89) podemos escrever 


$$
\begin{aligned}
\frac{I(\theta, \omega)}{I_{0}} & =\frac{\pi^{2} \Pi^{2}(\theta)}{\lambda^{4} R^{2}}\left\{\left(2 n_{0} \frac{d n}{d C_{B}} \frac{M}{N_{A v}}\right)^{2}\left\{1-\frac{R_{g}^{2} q^{2}}{3}\right\} \frac{C_{B} N_{a v} V}{M}\left\{\frac{1}{M}+2 A_{2} C_{B}\right\}^{-1}\right\} \\
\frac{I(\theta, \omega) R^{2}}{I_{0} \Pi(\theta)^{2}} & =\frac{4 \pi^{2} n_{0}^{2}}{\lambda^{2} N_{a v}} V\left(\frac{d n}{d C_{B}}\right)^{2}\left\{1-\frac{R_{g}^{2} q^{2}}{3}\right\}\left\{\frac{1}{M}+2 A_{2} C_{B}\right\}^{-1}
\end{aligned}
$$

Podemos agrupar algumas constantes

$$
K=\frac{4 \pi^{2} n_{0}^{2}}{\lambda^{2} N_{a v}}\left(\frac{d n}{d C_{B}}\right)^{2}
$$

onde $K$ refere-se as propriedades ópticas dos centros espalhadores, e

$$
\Delta R_{\theta}=\frac{I(\theta, \omega) / V R^{2}}{I_{0} \Pi^{2}(\theta)}
$$

onde $\Delta R_{\theta}$ é conhecida como razão Rayleigh, e normalmente é definida por unidade de volume, V. Na expressão (2.110) deixamos explicito a razão pelo volume.

Rearranjando a equação (2.108), podemos escrever a equação de Zimm

$$
\frac{K C_{B}}{\Delta R_{\theta}}=\left\{1+\frac{R_{g}^{2} q^{2}}{3}\right\}\left\{\frac{1}{M}+2 A_{2} C_{B}\right\}
$$

onde o primeiro termo do lado direito da igualdade refere-se a expansão no fator de forma e o segundo termo a expansão no fator de estrutura, ver por exemplo (Hiemenz, 1984).

Devemos lembrar que os parâmetros obtidos por espalhamento de luz correspondem a uma média ponderada pelas massas dos agregados ao quadrado. Essa média recebe o nome de $z_{\text {average }} \equiv z_{a v}$. Por exemplo, a massa molecular $M$, da expressão anterior (2.111), será substituída por uma massa molecular média, $\bar{M}_{w}$, que é obtida por meio expressão

$$
\bar{M}_{w}=\frac{\sum_{i} N_{i} M_{i}^{2} M_{i}}{\sum_{i} N_{i} M_{i}^{2}}
$$

onde $N_{i}$ corresponde ao número de agregados e $M_{i}$ a massa do agregado $i$. De forma análoga, para o raio de giração temos,

$$
R_{g}^{2}=\frac{\sum_{i} N_{i} M_{i}^{2} R_{g}^{2}}{\sum_{i} N_{i} M_{i}^{2}} .
$$

Deste modo, as partículas grandes exercem maior influência nos valores médios.

Os resultados das análises de Zimm podem ser observados no Capítulo 6. 



\section{Capítulo 3}

\section{Espalhamento de luz dinâmico}

\subsection{Introdução}

Este capítulo destina-se a explicar sucintamente a medida de espalhamento de luz dinâmico e as formas de análises utilizadas nesta dissertação.

\subsection{Função de Correlação}

Um processo estocástico, em geral, pode ser descrito por uma função de uma variável aleatória, $r$ e do tempo, $t, Y(r, t)$. A função de uma variável aleatória é também uma variável aleatória, e portanto deve ser definida por um conjunto de valores possíveis e uma distribuição de probabilidades.

No caso do Espalhamento de Luz Dinâmico, DLS (Dynamic Light Scattering), também conhecido como Espectroscopia de Correlação de Fótons, PCS ( Photon Correlation Spectroscopy), o fotodetector capta a intensidade da luz espalhada em função do tempo. A intensidade da luz espalhada é função da variável estocástica, $r$, que refere-se à distância dada entre a posição do centro espalhador no instante de tempo $t$ e a posição em um dado instante de tempo seguinte $t+\tau$. Quando o sistema se encontra em equilíbrio, a intensidade média pode ser tomada como uma média temporal, descrita por:

$$
\left\langle I\left(t_{0}, T\right)\right\rangle=\frac{1}{T} \int_{t_{0}}^{t_{0}+T} I(r, t) d t
$$

onde $t_{0}$ é o tempo em que a medida é iniciada e $T$ o tempo de realização da medida. Para a média ser representativa, o tempo de medida deve ser maior que o período de flutuação. Para um experimento ideal, $T \rightarrow \infty$, (Berne, 2000).

Em um processo estacionário, invariante por uma translação temporal, a média independe do tempo inicial: 


$$
\langle I\rangle=\lim _{T \rightarrow \infty} \frac{1}{T} \int_{0}^{T} I(t) d t
$$

A função de correlação é dada por

$$
g^{(2)}(\tau)=\lim _{T \rightarrow \infty} \frac{1}{T} \int_{0}^{T} I(t) J(t+\tau) d t .
$$

Para sistemas ergódicos, a média sobre o tempo pode ser substituída por uma média sobre as configurações, tal que

$$
\begin{aligned}
g^{(2)}(\tau) & =\langle I(t) J(t+\tau)\rangle=\lim _{T \rightarrow \infty} \frac{1}{T} \int_{0}^{T} I(t) J(t+\tau) d t \\
& =\langle I(0) J(\tau)\rangle
\end{aligned}
$$

onde $\langle\cdots\rangle$ denota uma média no ensemble de configurações.

A correlação de um processo estocástico pode ser definida por uma matriz de correlação, onde os termos diagonais correspondem aos termos de auto correlação, e os termos de fora da diagonal são chamados de correlações cruzadas. De modo análogo, se $I(t)$ e $J(t)$ correspondem a mesma função, $I(t)=J(t)$, ou seja, referem-se à mesma partícula, então $g^{(2)}(\tau)$ é chamada de função de auto correlação. Em sistemas diluídos admite-se que as correlações cruzadas são nulas. Portanto, em nossas análises estaremos interessados apenas na função de auto correlação.

Como podemos observar na figura 3.1, se o tempo é dividido em intervalos de tempo discretos de largura, $\Delta t$, onde $I_{n}$ corresponde a medida da intensidade no tempo $t_{0}+\tau$, em que $\tau=n \Delta t$, (Berne, 2000), e consequentemente $T=N \Delta t$, então podemos escrever:

$$
\langle I\rangle=\lim _{N \rightarrow \infty} \frac{1}{N} \sum_{j}^{N} I_{j}
$$

e

$$
g^{(2)}(\tau)=\langle I(0) I(\tau)\rangle=\lim _{N \rightarrow \infty} \frac{1}{N} \sum_{j}^{N} I_{j} I_{j+n}
$$

onde $I_{j}$ é a medida da intensidade no instante $t_{j}$, e $I_{j+n}$ é a medida da intensidade no instante $t_{j+n \Delta t}$, onde $n \Delta t=\tau$.

\subsection{Correlator}

Os fótons que atingem o detector são convertido em sinais elétricos. $\mathrm{O}$ correlator registra os pulsos elétricos coletados em intervalos de tempo discretos $\Delta t$, como podemos 


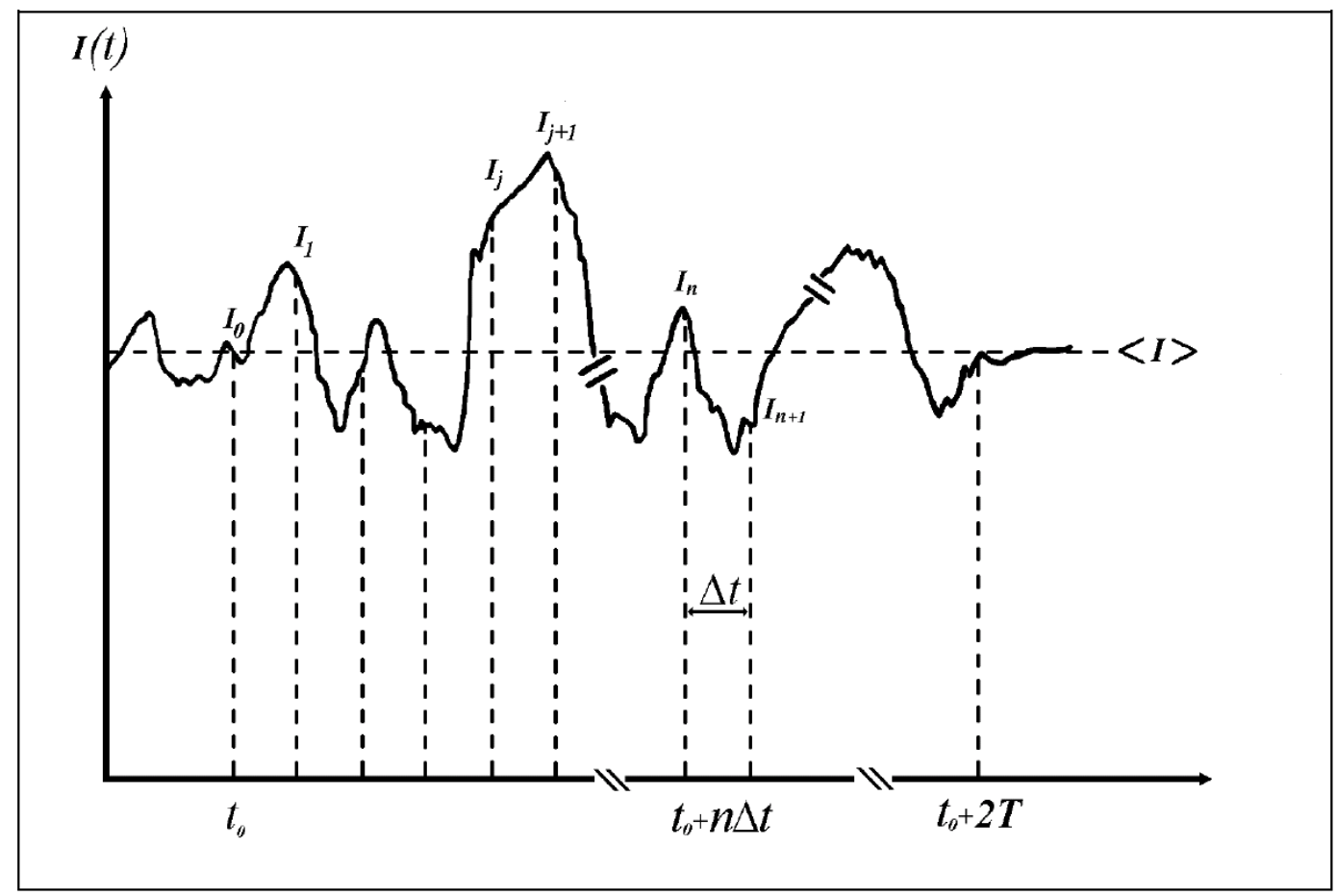

Figura 3.1: Flutuações da intensidade da luz espalhada no tempo, causada pelo movimento das moléculas no fluído.

observar na ilustração da figura 3.2, (Rodembusch, 2001). Por exemplo, no canal $n_{1}$ foi registrado um pulso e no canal $n_{2}$ foram registrados três pulsos.

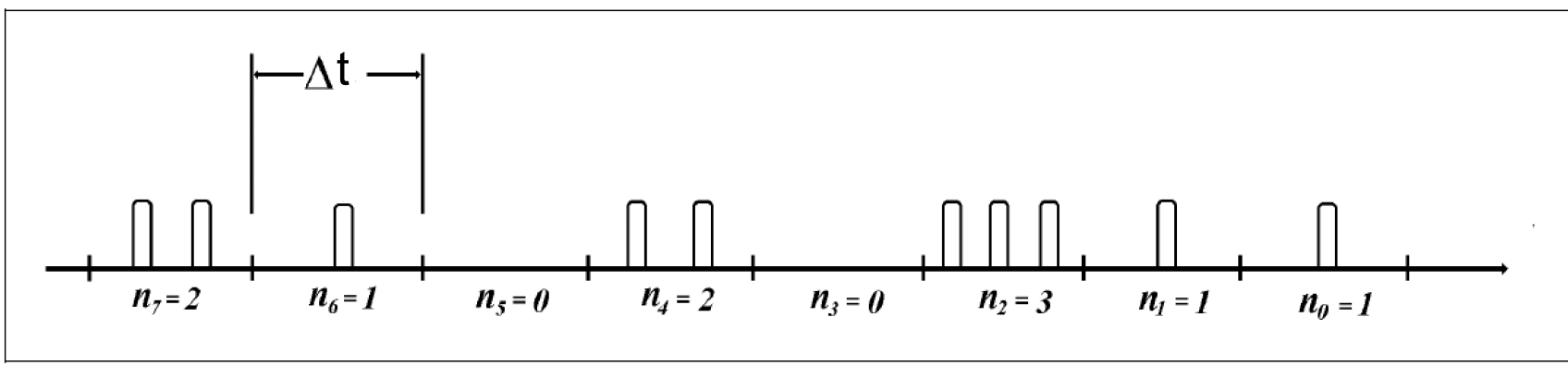

Figura 3.2: Trem de pulsos processados por um correlator.

O correlator possui um gerenciador de somas, que acumula a soma das correlações. Isto é, o correlator soma os produtos de pulsos elétricos obtidos do instante de tempo $t$ e de outros instantes de tempo $t+\tau$, onde $\tau$ assume os valores $n \Delta t$. Por exemplo, a correlação do canal $n_{1}$ com o canal $n_{2}$ será dada por $n_{1} \cdot n_{2}=6$. O intervalo de tempo entre as medidas dos canais $n_{1}$ e $n_{2}$, corresponde a $\Delta t$. O intervalo de tempo seguinte, 
será dado por $2 . \Delta t$, e portanto a correlação é estabelecida entre o canal $n_{1}$ e $n_{3}$, por exemplo.

O gerenciado de somas acumula todas as correlações possíveis, dos dados que foram registrados. Então uma expressão geral para representar a soma de todas as correlações é dada por

$$
\begin{aligned}
C\left(\tau_{j}\right) & =\lim _{N \rightarrow \infty} \frac{1}{N} n_{0} \cdot n_{k}+n_{1} \cdot n_{k}+\ldots \\
& =\lim _{N \rightarrow \infty} \frac{1}{N} \sum_{i}^{N} n_{i} \cdot n_{i+j},
\end{aligned}
$$

onde $j=1,2,3, \ldots$, e esta, consiste em uma boa aproximação para a função de correlação, como vimos na equação (3.6), (Rodembusch, 2001).

\subsection{Análises na função de correlação do campo elétrico}

A função de correlação do campo elétrico, $g^{(1)}(\tau)$, pode ser obtida a partir $g^{(2)}(\tau)$ segundo a equação de Siegert, para sistemas diluídos, (Brown, 1993)

$$
g^{(2)}(\tau)=\beta\left(1+\left|g^{(1)}(\tau)\right|^{2}\right),
$$

onde $\beta$ depende de condições instrumentais e refere-se à linha de base.

A relação de Siegert é violada nos casos onde há poucas partículas no volume de espalhamento, ou quando o sistema não é ergódico.

A função de correlação $g^{(1)}(\tau)$ pode ser relacionada a um decaimento exponencial, quando o sistema é monodisperso

$$
g^{(1)}(\tau) \sim \exp (-\Gamma \tau)
$$

onde $\Gamma$ é a taxa de decaimento da função e está relacionada a uma frequência de relaxação do movimento da partícula, e $\tau$ corresponde ao intervalo de tempo em que as correlações são estabelecidas. No entanto, para sistemas polidispersos a função de autocorrelação contém a informação de diversos decaimentos, referentes aos tamanhos variados da dispersão. Para separar os diferentes decaimentos são necessários programas e métodos adequados.

Há diversos métodos de ajuste para função de correlação, $g^{(1)}(\tau)$. Entre eles, um dos mais utilizados é o método dos cumulantes, (Koppel, 1972), segundo o qual

$$
g^{(1)}(\tau)=A \exp \left\{-\Gamma \tau\left(1+\frac{\mu_{2}}{2 !} \tau^{2}-\frac{\mu_{3}}{3 !} \tau^{3}+\cdots\right)\right\}
$$


onde $\mu_{n}$ corresponde aos momentos, ou cumulantes, da expansão. A expansão dos cumulantes, pode ser vista com mais detalhe no apêndice $(\mathrm{A})$.

O primeiro momento fornece um valor esperado para a taxa de decaimento $\Gamma$, que está relacionado ao coeficiente de difusão translacional médio dos centros espalhadores, $D_{T}$,

$$
\Gamma=D_{T} q^{2}
$$

onde $q$ corresponde ao vetor de espalhamento.

Logo o $D_{T}$ pode ser obtido segundo a relação

$$
D_{T}=\lim _{\Delta q \rightarrow 0} \frac{\Delta \Gamma}{\Delta q^{2}} \equiv \frac{d \Gamma}{d q^{2}},
$$

onde $\frac{d \Gamma}{d q^{2}}$ corresponde ao coeficiente angular da reta $\Gamma=D_{T} q^{2}$.

O segundo momento da expansão está relacionado com a variância da distribuição (índice de polidispersão), conforme o apêndice A.

Essas análises foram feitas no programa de controle 9KDLSW, da Brookhaven.

Também utilizamos uma outra análise, que faz o ajuste a $g^{(1)}(\tau)$ de forma diferente. O Contin é um algoritmo que ajusta à função de correlação uma distribuição de exponenciais, que pode ser representada por uma integral de Laplace.

$$
g^{(1)}(\tau)=\int_{0}^{\infty} A\left(\Gamma^{-1}\right) \exp \left(-\Gamma^{-1} \tau\right) d \Gamma
$$

Através da transformada de Laplace inversa, o algoritmo obtém a função de distribuição da taxa de decaimento, $A\left(\Gamma^{-1}\right)$, onde $\Gamma^{-1}$ corresponde ao tempo de relaxação $\tau_{r}{ }^{1}$ e com o uso das equações 3.11 e novamente, com o uso da equação 3.11 pode ser obtido o $\bar{D}_{T}$. Essa análise fornece uma amostragem de decaimentos exponenciais, enquanto o método do Cumulantes, fornece um decaimento médio, e seu desvio médio. As análises utilizando programa Gendist, algoritmo REPES (Jakes, 1995)

O coeficiente de difusão translacional está sujeito à ação das interações envolvidas no sistema. Na presença de interações entre os centros espalhadores a difusão é influenciada pela ação de forças externas, por isso convém calcular um coeficiente de difusão em um regime de diluição infinita, (Berne, 2000; Brown, 1993). Deste modo, $D_{T}$ pode ser escrito sob a forma

$$
D_{T}=D_{0}\left(1+k_{D} C\right)
$$

\footnotetext{
${ }^{1} \mathrm{O}$ tempo de relaxação $\tau_{r}$ está relacionado com a relaxação do movimento e é diferente do "delay time" $\tau$, que corresponde aos intervalos de tempos que são estabelecidas as correlações.
} 
onde $D_{0}$ corresponde ao coeficiente de difusão no regime de diluição infinita, isto é, livre da ação das interações impostas pelo sistema, $k_{D}$ é denominado coeficiente de concentração, (Brown, 1993) e $C$ corresponde à concentração dos centros espalhadores.

Uma vez obtido o coeficiente de difusão dos centros espalhadores, livre de interações com relação aos demais centros (regime de diluição infinita) é possível determinar o diâmetro hidrodinâmico das partículas, $d_{e f}$ assumidas esferas, de acordo com a equação de Stokes-Einstein,

$$
D_{0}=\frac{k_{B} T}{3 \pi \eta d_{e f}}
$$

onde $k_{B}$ é a constante de Boltzmann e $\eta$ a viscosidade do solvente.

O diâmetro efetivo obtido por DLS, nas análises que apresentamos acima, fornecem valores médio obtidos pelo $z_{a v}$, deste modo,

$$
d_{e f}=\frac{\sum_{i} N_{i} M_{i}^{2} d_{i}}{\sum_{i} N_{i} M_{i}^{2}}
$$

Portanto as partículas grandes das dispersão exercem influência muito maior no valor médio obtido.

Os resultados de DLS podem ser observados no Capítulo 6 . 


\section{Capítulo 4}

\section{Materiais e métodos}

\section{Materiais}

\section{Lipídios}

Dimiristoil fosfatidilglicerol (DMPG) (Avanti Polar Lipids, Inc.) - Dimiristoil fosfatidilcolina (DMPC) (Avanti Polar Lipids, Inc.)

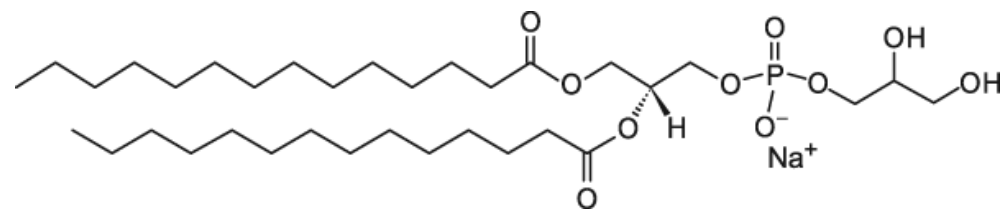

Figura 4.1: DMPG

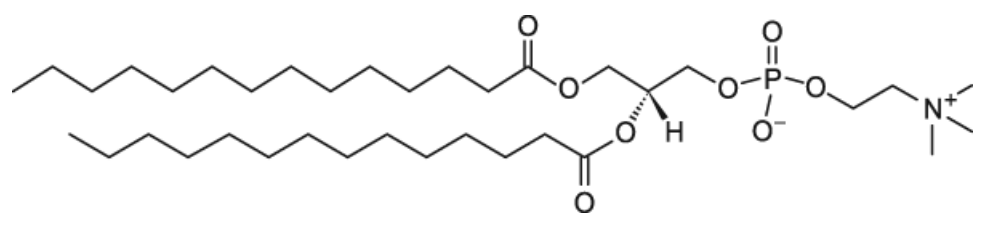

Figura 4.2: DMPC

\section{Solventes}

Tampão Hepes: (N-[2-hidroxietil]piperazine-N'-[2-etanosulfonicoacido]) (HEPES) (Sigma Chemical), $10 \mathrm{mM}$, baixa força iônica, ácido etilenodiamido tetra-acético (EDTA), 1, 0 mM- (Sigma Chemical). O pH da solução foi ajustado com hidróxido de sódio $\mathrm{NaOH}$ em $\mathrm{pH}=7,41$.

Clorofórmio $\left(\mathrm{CHCl}_{3}\right)$, P.A. ACS - (Vetec química fina). Utilizado para preparação de filmes lipídicos. 
Água Mili-Q - Mili-Q plus ultra pure water system - Millipore.

\section{Reagentes}

Álcool isopropílico $\left(\mathrm{C}_{3} \mathrm{H}_{8} \mathrm{O}\right)$ - (Casa Americana). Utilizado no refratômetro diferencial, para evitar acúmulo de resíduos e bactérias nos canais e compartimentos destinados ao solvente e a solução.

Decalina $\left(\mathrm{C}_{10} \mathrm{H}_{18}\right)$, decahidronaftaleno, anidro, 99\% - (Sigma Chemical). Utilizado na cuba do goniômetro (espalhamento de luz), a solução fica diretamente em contato com o porta-amostra. A Decalina, possui índice de refração semelhante ao do vidro, e é utilizada para evitar a refração do vidro (porta-amostra e cuba).

Ácido perclórico $\left(\mathrm{HClO}_{4}\right)$ - (Sigma Chemical). Utilizado na dosagem de fosfato.

Molibidato de amônio $\left(\left(\mathrm{NH}_{4}\right)_{6} \mathrm{Mo}_{7} \mathrm{O}_{24} \cdot \mathrm{H}_{2} \mathrm{O}\right)$ - (Vetec química fina). Utilizado na dosagem de fosfato.

Ácido ascórbico $\left(\mathrm{C}_{6} \mathrm{H}_{8} \mathrm{O}_{6}\right)$ - (Sigma Chemical). Utilizado na dosagem de fosfato.

\section{Métodos}

\section{Preparação de vesículas}

Para preparação de vesículas, os lipídios são diluídos em clorofórmio e depois submetidos a um fluxo de nitrogênio, $N_{2}$, levando à formação de um filme na parede do tudo de ensaio. O filme é então submetido a baixa pressão por três horas. Em seguida é adicionado o tampão e a suspensão é agitada em vórtex. As dispersões lipídicas são posteriormente extrusadas.

\section{Vesículas extrusadas}

Com o uso de um extrusor (Avanti Polar Lipids), passamos as vesículas por um filtro de policarbonato (Nuclepore). Utilizamos filtros com poros de tamanho equivalente a $100 \mathrm{~nm}$. Esse processo foi adotado com a finalidade de reduzir o índice de dispersão dos agregados lipídicos. 


\section{Absorção óptica: dosagem de fosfato}

Utilizamos um espectrofotômetro Varian - Carry 50 Bio (UV- visível), para medir a absorbância das amostras, definida na equação 5.4.

Medidas de absorção óptica foram realizadas para determinar a concentração de lipídios das dispersões de DMPG e DMPC, dosando-se a quantidade de fosfato das amostra utilizadas nas análises de Zimm. O processo de dosagem de fosfato (Rousser et al, 1970) está resumido a seguir.

1. Separa-se uma alíquota $10 \mu L$ das soluções que desejamos determinar a concentração em um tubo de ensaio $(5 \mathrm{~mL})$.

2. Estabelece-se uma curva de calibração a partir de 5 tubos (referências) com uma solução de tampão fosfato, (Pi), a 1, 0 mM. Cada tubo continha 10, 30, 50, 70 e $90 \mu L$ do tampão, que corresponde a 10, 30, 50, 70 e 90 n mols de Pi. A solução que se deseja determinar a concentração deve conter números de mols dentro do intervalo descrito pela curva de calibração. Para cada dosagem de fosfato realizada, fizemos mais de uma curva de calibração e tomamos a média das curvas.

3. Deixa-se as amostras e as referências em uma estufa (Quimis) a $120^{\circ} \mathrm{C}$, para secar o conteúdo dos tubos.

4. A partir desse passo, incluí-se uma referência que não contem fosfato, que servirá de branco nas leituras do espectrofotômetro.

5. Adiciona-se 0,4 $\mathrm{mL}$ de ácido hiperclórico, $\mathrm{HClO}_{4}$, concentrado 70\%. Colocamos os tubos para digerir em um bloco quente (Stuart Science) a $180^{\circ} \mathrm{C}$, e tampamos com bolinhas de gude para minimizar efeitos da evaporação. Essa condição foi mantida por 1 hora.

6. Após esfriar, adiciona-se 1, $0 \mathrm{~mL}$ de água destilada, e $0,4 \mathrm{~mL}$ de molibidato de amônio $1,25 \%(\mathrm{p} / \mathrm{v})$ e misturamos em vórtex.

7. Em seguida, adiciona-se 0, $4 \mathrm{~mL}$ de ácido ascórbico $3 \%(\mathrm{p} / \mathrm{v})$ e mistura-se em vórtex, novamente.

8. Deixa-se os tubos em banho térmico com água fervente, por cerca de 10 minutos, para a formação do composto que absorve luz em $\lambda=797, \mathrm{~nm}$.

9. Após as amostras esfriarem, lê-se a absorbância de cada amostra em um espectrofotômetro, $(\lambda=797 \mathrm{~nm})$, subtraindo-se o branco. 
10. As leitura das referências nos fornecem as curvas de calibração, e leituras das amostras que desejamos determinar a concentração são comparadas com aos valores obtidos na curva de calibração, e assim pode-se determinar o número de mols que a alíquota inicial continha. Os resultados da dosagem de fosfato podem ser observados na seção 5.1.2.

\section{Refratômetro diferencial}

Utilizando o refratômetro diferencial da Brookhaven, e com auxílio do programa de aquisição BI-DNDCW Differential Refratometer, foram realizadas as análises para obter o incremento no índice de refração, $d n / d c$, conforme será discutido no Capítulo 5 . A calibração do refratômetro diferencial foi realizada com cloreto de potássio, $K C l$, para as seguintes temperaturas $T=20,28$ e $45^{\circ} \mathrm{C}$, com $50 \%$ da intensidade da fonte luminosa. Foram preparadas cinco amostras de diferentes concentrações, de DMPC e DMPG, a partir da diluição da dispersão lipídica extrusada mais concentrada.

\section{Calorimetria Diferencial de Varredura}

As medidas de calorimetria foram desenvolvidas no aparelho Microcal VP-DSC. A velocidade de varredura de aquecimento, ("scan rate") utilizada nas medidas corresponde a $20^{\circ} \mathrm{C} / \mathrm{h}$. As análises da calorimetria diferencial de varredura (DSC) foram feitas no programa Microcal Oringin 7.0. A curva de DSC da amostra (dispersão lipídica) foi subtraída da curva obtida para o sistema de referência (tampão). A linha de base é ajustada com o uso deste mesmo programa e foi utilizado um polinômio de terceiro grau para este feito. A entalpia do processo de transição de fase é calculada através da área sob a curva obtida de $C_{p}$ em função da temperatura.

\section{Espalhamento de luz}

As medidas de espalhamento de luz foram realizadas em um equipamento da Brookhaven, composto pelas seguintes unidades: auto-correlator digital BI-9000AT, foto multiplicadora BI-9025AT e goniômetro BI-200S, o qual é acoplado a um banho térmico (Poly Science). A temperatura no interior da cuba (que contém o porta-amostra) é monitorada com auxilio de um termopar (Fluke). O equipamento dispõe de programas para a aquisição de dados e controles de alinhamento. Alguns programas estão listados a se- 
guir: BI-ISTW, para alinhamento do goniômetro; BI-ZPW para a análise de Zimm, e o programa de controle do auto-correlator digital 9KDLSW.

Para a análise de Zimm são preparadas cinco amostras de diferentes concentrações, partindo-se da diluição da dispersão lipídica extrusada mais concentrada, conforme realizado para o refratômetro diferencial. Previamente realizamos um teste de alinhamento para definir uma região angular ideal para trabalho. Definida a região angular que está alinhada com toda a parte óptica do goniômetro e com o laser, a intensidade da luz espalhada é então coletada a diversos ângulos. Os gráficos de Zimm foram obtidas em várias temperaturas.

Medidas de espalhamento de luz dinâmico também foram realizadas nas mesmas concentrações e temperaturas, em no mínimo quatro ângulos diferentes. Utilizamos dois métodos de análise para as funções de auto-correlação, o método dos Cumulantes, com correções até a segunda ordem, (9KDLSW - Brookhaven) e também o método Contin, do programa Gendist, (Jakes, 1995). 



\section{Capítulo 5}

\section{Técnicas experimentais complementares}

\subsection{Absorção óptica}

$\mathrm{Na}$ interação da radiação com a matéria pode ocorrer o fenômeno conhecido como absorção óptica (região visível/UV). A absorção da radiação é estimulada quando a energia transportada pelos fótons incidentes coincidem com a diferença entre níveis de energia moleculares (ressonância). A energia absorvida promove, portanto, transições eletrônicas para níveis de maior energia, (ver por exemplo (Sakurai, 1994)).

Para o propósito deste trabalho, a abordagem clássica da absorção óptica é suficiente para o desenvolvimento e entendimento dos resultados que serão apresentados a seguir.

\subsubsection{Lei de Lambert-Beer}

A Lei de Lambert-Beer pode ser deduzida de uma maneira simples, com o auxílio do seguinte modelo. Consideramos um feixe de luz, transportando energia, com intensidade $I_{0}$, conforme a figura 5.1.

O feixe incide em uma amostra contida em um recipiente de caminho óptico $L$. O diferencial de intensidade, $d I$, ao longo do caminho percorrido pela luz, pode ser escrito do seguinte modo

$$
d I(y)=-\mathcal{E}^{\prime} C I(y) d y
$$

onde $\mathcal{E}^{\prime}$ corresponde a uma constante que refere-se a características intrínsecas da molécula absorvedora, e $C$ refere-se à concentração de centros absorvedores. Portanto 


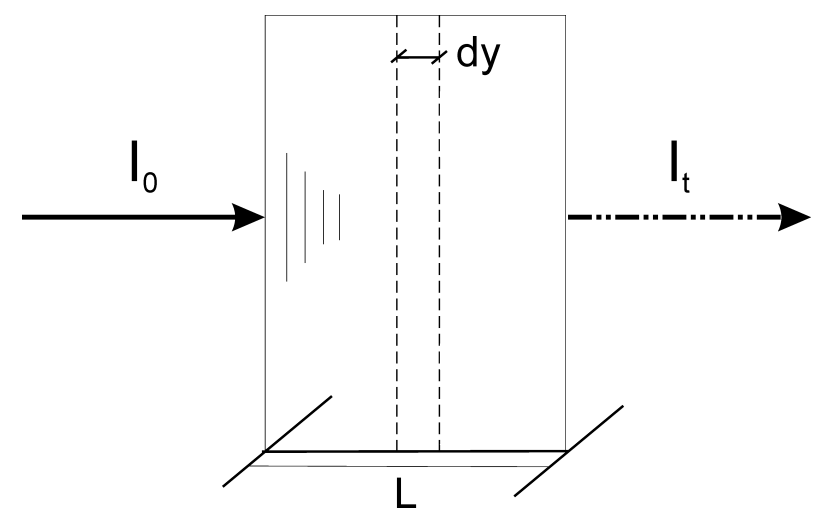

Figura 5.1: Esquema ilustrativo do modelo proposto para deduzir a Lei de Lambert-Beer.

assume-se que a intensidade da luz absorvida é proporcional a $C$.

Integrando a relação anterior 5.1, nos limites de intensidade $I_{t}$ e $I_{0}$, visto que $I_{t}<I_{0}$, e no caminho percorrido pela luz, temos,

$$
\ln \left(\frac{I_{0}}{I_{t}}\right)=\mathcal{E}^{\prime} C L
$$

Define-se, portanto, uma grandeza que relaciona a intensidade da luz incidente com a intensidade da luz transmitida, chamada absorbância $A$,

$$
A \equiv \log \left(\frac{I_{0}}{I_{t}}\right)
$$

Fazendo uma mudança na base logarítmica da equação 5.2, temos:

$$
A=\underbrace{\mathcal{E}^{\prime} / \ln 10}_{\mathcal{E}} C L
$$

logo, a lei de Lambert-Beer pode ser escrita sob a forma,

$$
A=\mathcal{E} C L
$$

onde $\mathcal{E}$ é conhecido como coeficiente de extinção molar, e $C$ é dado em mol/L. 


\subsubsection{Dosagem de fosfato: Determinação da concentração de fosfolipídios.}

As medidas de absorção óptica foram realizadas para determinar as concentrações exatas das dispersões lipídicas de DMPG e DMPC, dosando a quantidade de fosfato das amostras utilizadas na análise de Zimm.

A dosagem de fosfato exige uma curva de calibração prévia, estabelecida a partir de soluções de tampão fosfato, como discutido anteriormente.

A curva de calibração relaciona diferentes quantidades de fosfato, número de moles (n Pi), com a absorbância medida. Então, primeiramente as alíquotas da substância que se deseja determinar a concentração, passam por um processo químico, como descrito no Capítulo 4, Materiais e Métodos.

Em seguida, medimos a absorbância das soluções obtidas, e por meio da curva de calibração determinamos o número de moles de fosfato que a alíquota inicial continha, e por fim pode-se calcular as concentrações das amostras.

Os resultados obtidos da dosagem de fosfato de amostras de DMPG e DMPC são apresentados a seguir.

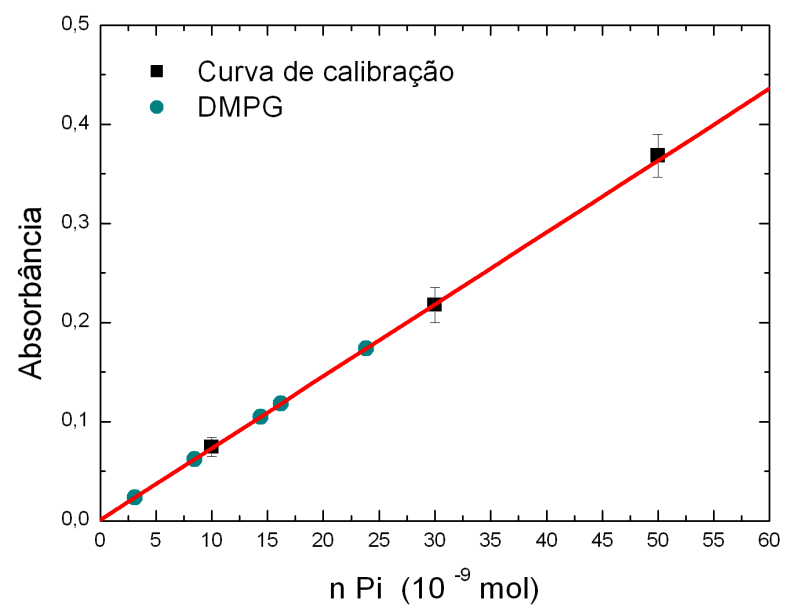

Figura 5.2: Dosagem de fosfato do DMPG, conjunto de amostras 1.

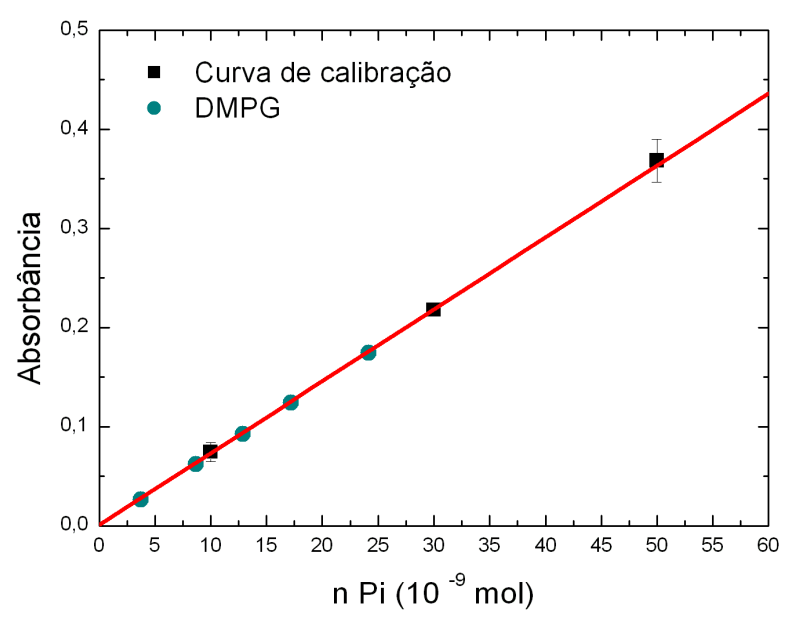

Figura 5.3: Dosagem de fosfato do DMPG, conjunto de amostras 2 .

As concentrações, $C$, obtidas pela dosagem de fosfato do DMPG, estão resumidas na tabela 5.1 . 
Tabela 5.1: Concentrações obtidas da dosagem de fosfato, em (mM) e em $(\mathrm{mg} / \mathrm{mL})$, para dois conjuntos de amostras de DMPG.

\begin{tabular}{|c|||c||c||c||c|}
\hline $\begin{array}{c}C(\mathrm{mM}) \\
\text { nominal (preparada) }\end{array}$ & $\begin{array}{c}C(\mathrm{mM}) \\
\text { figura 5.2 }\end{array}$ & $\begin{array}{c}C(\mathrm{mg} / \mathrm{mL}) \\
\text { figura 5.2 }\end{array}$ & $\begin{array}{c}C(\mathrm{mM}) \\
\text { figura 5.3 }\end{array}$ & $\begin{array}{c}C(\mathrm{mg} / \mathrm{mL}) \\
\text { figura 5.3 }\end{array}$ \\
\hline \hline 0,50 & 0,31 & 0,214 & 0,37 & 0,255 \\
\hline 1,00 & 0,84 & 0,579 & 0,87 & 0,599 \\
\hline 1,50 & 1,44 & 0,992 & 1,29 & 0,889 \\
\hline 2,00 & 1,62 & 1,11 & 1,72 & 1,19 \\
\hline 2,50 & 2,38 & 1,64 & 2,41 & 1,66 \\
\hline \hline
\end{tabular}

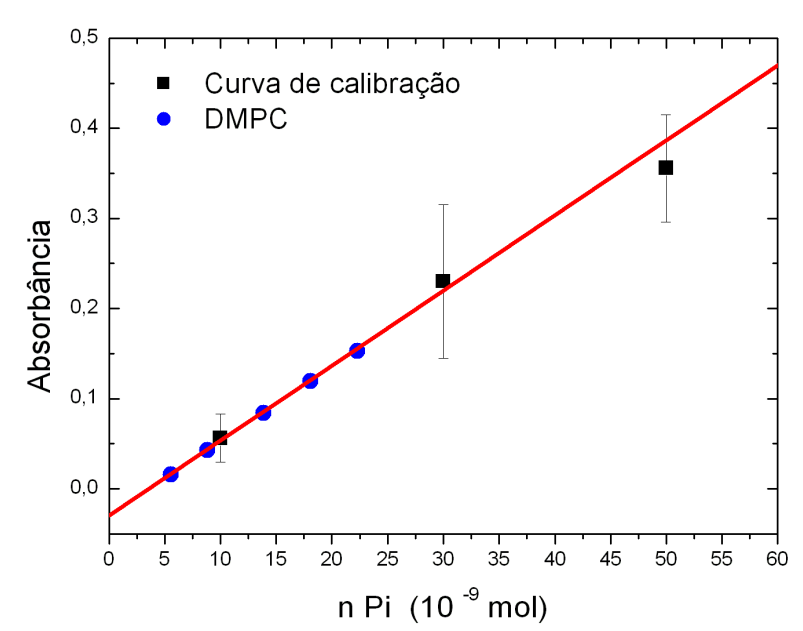

Figura 5.4: Dosagem de fosfato do DMPC, conjunto de amostras 1.

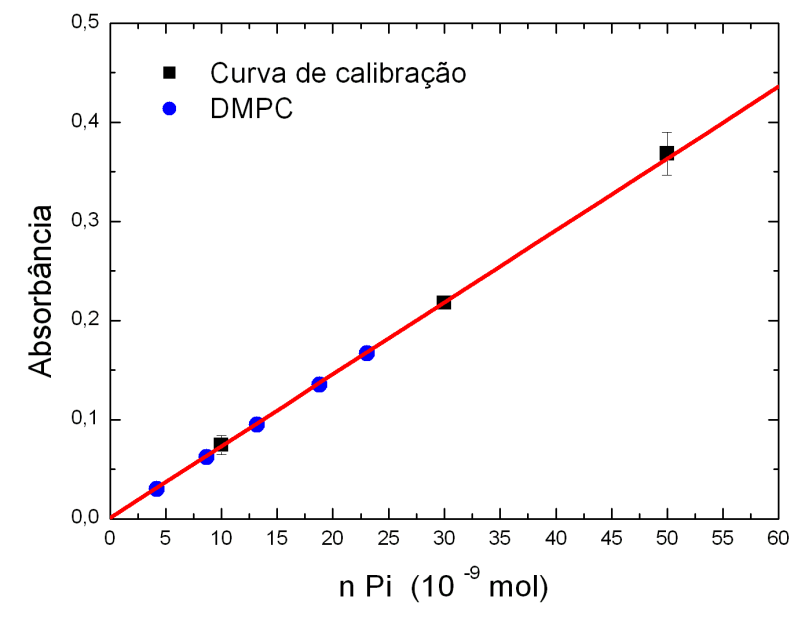

Figura 5.5: Dosagem de fosfato do DMPC, conjunto de amostras 2 .

Podemos observar as concentrações obtidas pela dosagem de fosfato do DMPC na tabela 5.2.

Tabela 5.2: Concentrações obtidas da dosagem de fosfato, em (mM) e em $(\mathrm{mg} / \mathrm{mL})$, para dois conjuntos de amostras de DMPC.

\begin{tabular}{|c||c||c||c||c|}
\hline $\begin{array}{c}C(\mathrm{mM}) \\
\text { nominal (preparada) }\end{array}$ & $\begin{array}{c}C(\mathrm{mM}) \\
\text { figura 5.2 }\end{array}$ & $\begin{array}{c}C(\mathrm{mg} / \mathrm{mL}) \\
\text { figura 5.2 }\end{array}$ & $\begin{array}{c}C(\mathrm{mM}) \\
\text { figura 5.3 }\end{array}$ & $\begin{array}{c}C(\mathrm{mg} / \mathrm{mL}) \\
\text { figura 5.3 }\end{array}$ \\
\hline \hline 0,50 & 0,55 & 0,373 & 0,42 & 0,285 \\
\hline 1,00 & 0,88 & 0,597 & 0,87 & 0,590 \\
\hline 1,50 & 1,39 & 0,942 & 1,32 & 0,895 \\
\hline 2,00 & 1,81 & 1,23 & 1,88 & 1,28 \\
\hline 2,50 & 2,23 & 1,51 & 2,31 & 1,57 \\
\hline \hline
\end{tabular}

Os resultados obtidos nas tabelas 5.1 e 5.2 serão utilizados nas análises de Zimm. 


\subsection{Refratômetro Diferencial}

A intensidade da luz espalhada é proporcional à polarizabilidade molecular, como vimos na equação 2.67. A expressão de Claussius-Mossoti, relação 2.103, relaciona a polarizabilidade molecular com o índice de refração da solução, e a variação do índice de refração da solução em relação à variação da concentração de soluto, que podem ser medidas experimentalmente com o uso de um refratômetro diferencial.

O refratômetro diferencial pode ser esquematizado pela figura 5.6.

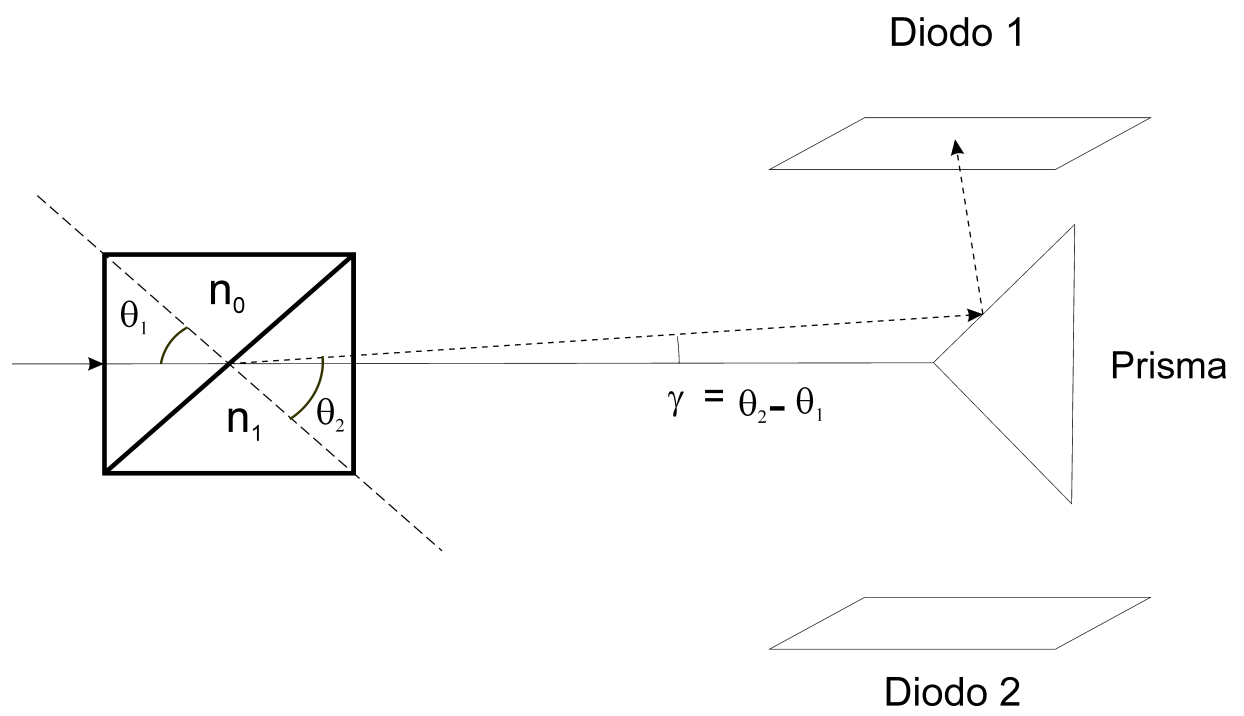

Figura 5.6: Esquema ilustrativo do refratômetro diferencial.

Como ilustra a figura, o refratômetro diferencial possui dois compartimentos: um destinado ao solvente, de índice de refração $n_{0}$ e outro destinado à amostra, de índice de refração $n_{1}$. O feixe do laser atravessa os dois compartimentos. Caso os dois compartimentos estejam preenchidos com o solvente o feixe não será desviado. Entretanto, se o compartimento destinado a amostra contiver uma solução com índice de refração diferente do solvente, o feixe do laser apresentará um pequeno desvio e atingirá uma das faces do prisma, conforme a figura. O feixe refletido pelo prisma será coletado por um diodo, onde o sinal luminoso será convertido em um sinal elétrico. Portanto, o ganho na voltagem, $\Delta V_{g}$, será proporcional ao incremento no índice de refração.

É necessário estabelecer uma curva de calibração com uma solução padrão, com índice de refração conhecido em diversas concentrações. Utilizamos solução de $K C l$ para obter a curva de calibração. Preparamos 10 soluções de $K C l$ com as seguintes concentrações 1,$0 ; 2,0 ; 3,0 ; 4,0 ; 5,0 ; 6,0 ; 7,0 ; 8,0 ; 9,0$ e 10,0 (mg/mL). As amostras foram preparadas individualmente, pesando as devidas quantidades de sal e solvente adicionado, obtendo 
um erro na concentração (estimado) inferior a $0,1 \%$.

Durante o processo de calibração, cada concentração de $\mathrm{KCl}$ é associada a um determinado ganho na voltagem, $V_{g}$, como mostra o gráfico da figura 5.7, por exemplo. Como os índices de refração desta solução padrão são conhecidos, então, os ganhos $V_{g}$ são associados às variações do índice de refração da solução $\Delta n=n_{s}-n_{0}$, onde $n_{0}$ é o índice de refração do solvente e $n_{s}$ é o índice de refração do soluto. A equação da reta obtida na figura 5.8, possui coeficiente angular dado pela constante de calibração $K$, e coeficiente linear praticamente nulo, $O\left[\sim 10^{-16}\right]$.

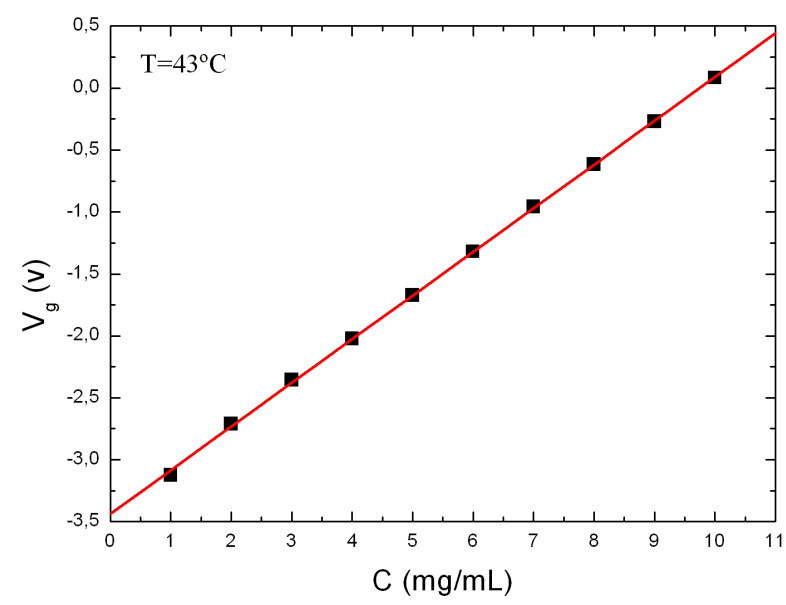

Figura 5.7: Curva de calibração: Ganho na voltagem com relação à concentração, para o $K C l$ a $43^{\circ} \mathrm{C}$.

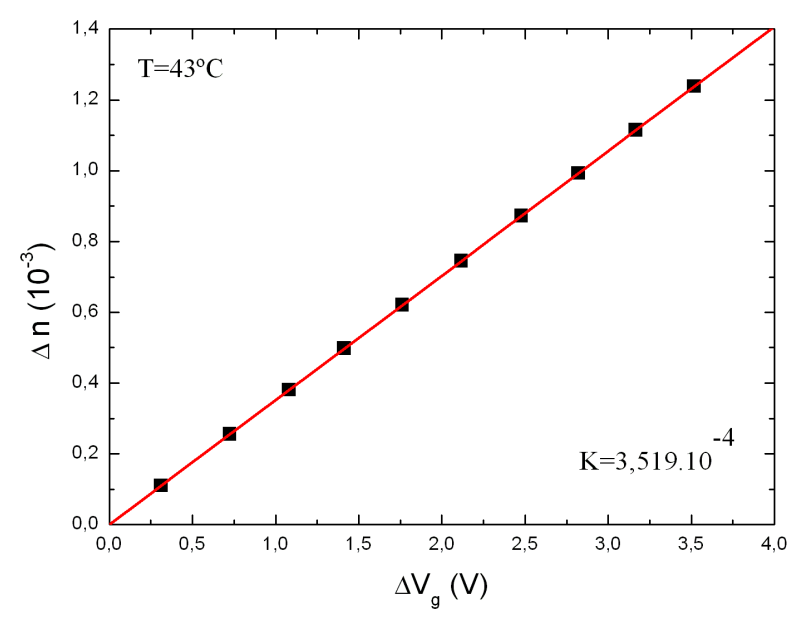

Figura 5.8: Curva de calibração: Incremento do índice de refração com relação à concentração, para o $\mathrm{KCl}$ a $43^{\circ} \mathrm{C}$.

Estabelecida a curva de calibração, novas curvas que relacionam concentração de soluto e $V_{g}$ podem ser medidas. Vamos tomar como exemplo o DMPG na fase fluída em $T=$ $43^{\circ} \mathrm{C}$, conforme a figura 5.9. Então, os valores de $V_{g}$ medidos podem ser relacionados às variações do índice de refração da solução por meio da curva de calibração, e assim é estabelecida a curva dada pela figura 5.10. 


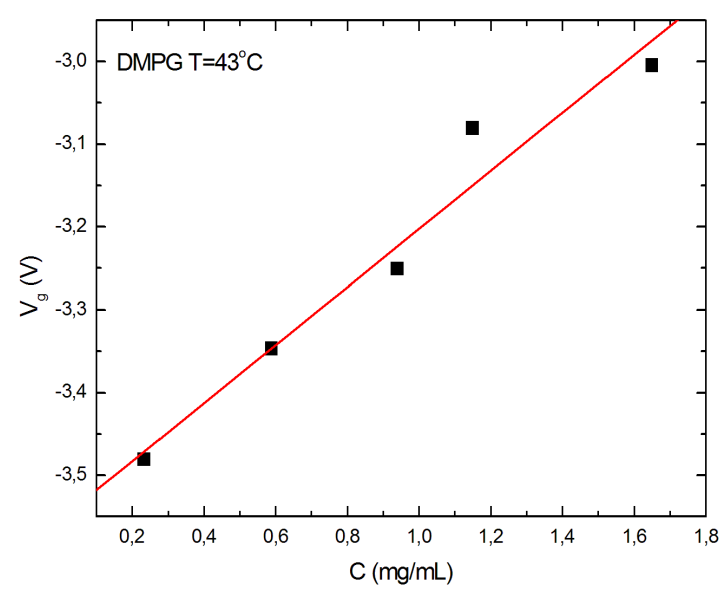

Figura 5.9: Ganho na voltagem com relação à concentração, para o DMPG a $43^{\circ} \mathrm{C}$.

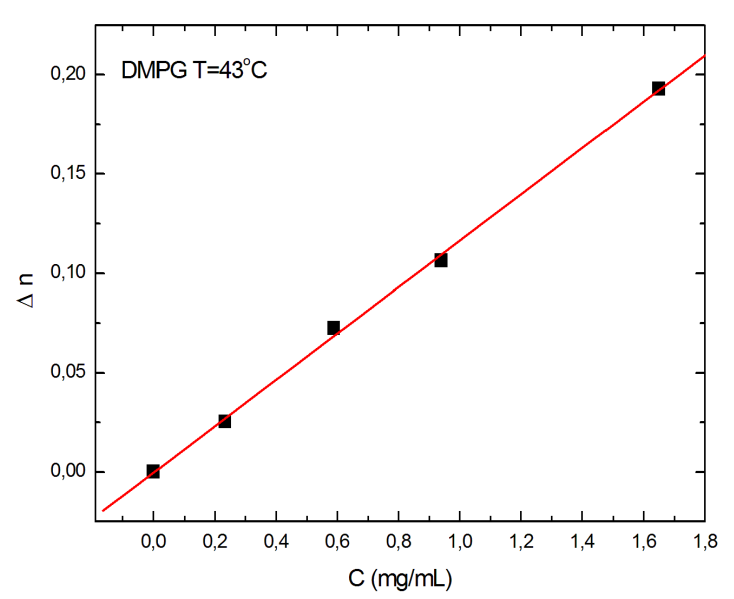

Figura 5.10: Incremento do índice de refração com relação à concentração, para o $\mathrm{DMPG}$ a $43^{\circ} \mathrm{C}$.

O incremento no índice de refração é calculado segundo a relação

$$
\lim _{\Delta c \rightarrow 0} \frac{\Delta n}{\Delta c}=\frac{d n}{d c}
$$

que corresponde ao coeficiente angular da reta obtida na figura 5.10.

Cada temperatura exige uma nova curva de calibração. Para $T=20^{\circ} \mathrm{C}$, o ganho na voltagem para cada concentração de $K C l$, e a curva de calibração dada pela relação de $\Delta n$ e $\Delta V_{g}$, são apresentados nas figuras 5.11 e 5.12 , respectivamente.

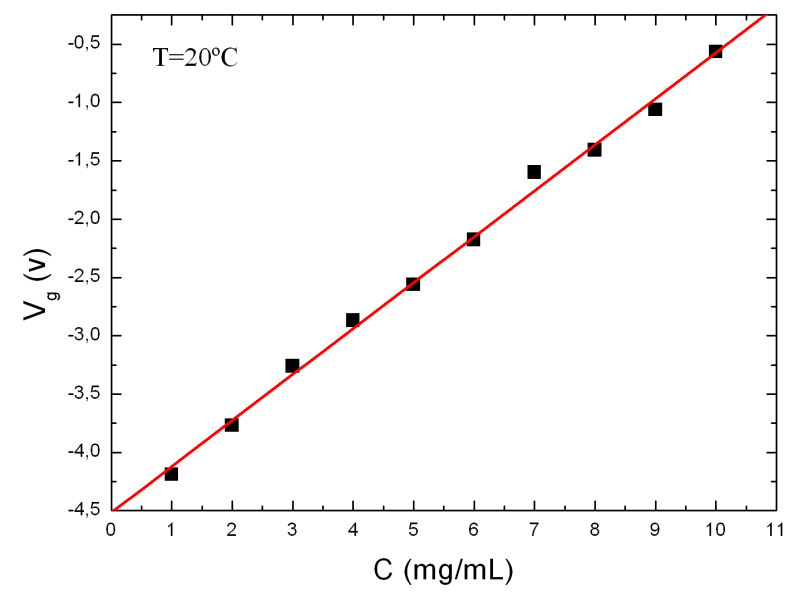

Figura 5.11: Curva de calibração: Ganho na voltagem com relação à concentração, para o $K C l$ a $20^{\circ} \mathrm{C}$.

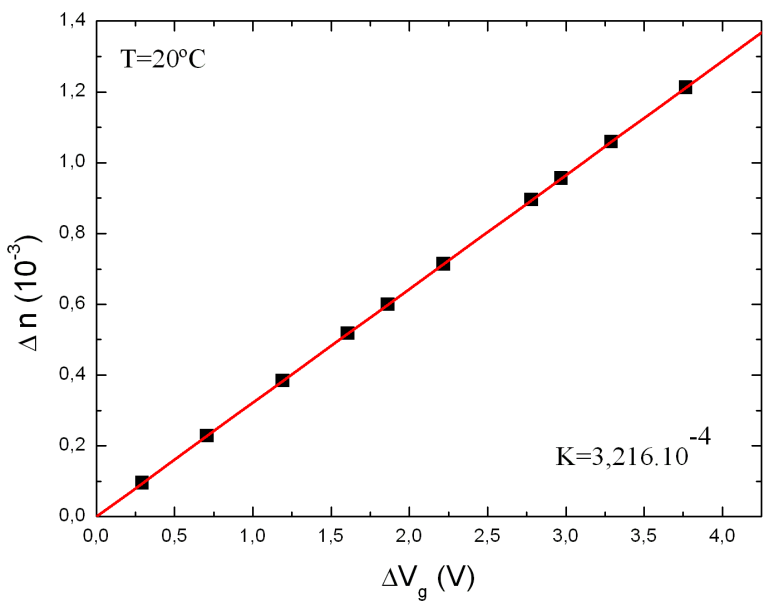

Figura 5.12: Curva de calibração: Incremento do índice de refração com relação à concentração, para o $\mathrm{KCl}$ a $20^{\circ} \mathrm{C}$.

O mesmo é válido para $T=28^{\circ} \mathrm{C}$, onde a curva de calibração pode ser observada conforme a figura 5.14. 


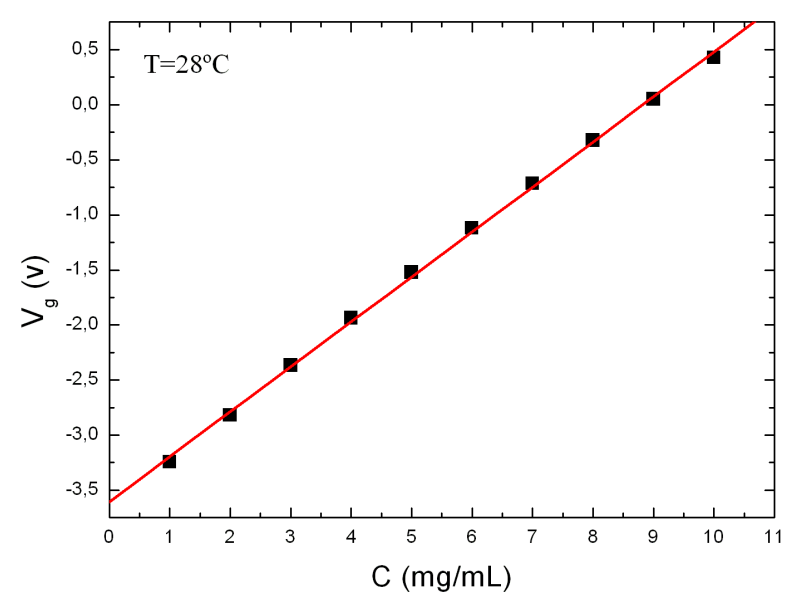

Figura 5.13: Curva de calibração: Ganho na voltagem com relação à concentração, para o $\mathrm{KCl}$ a $28^{\circ} \mathrm{C}$.

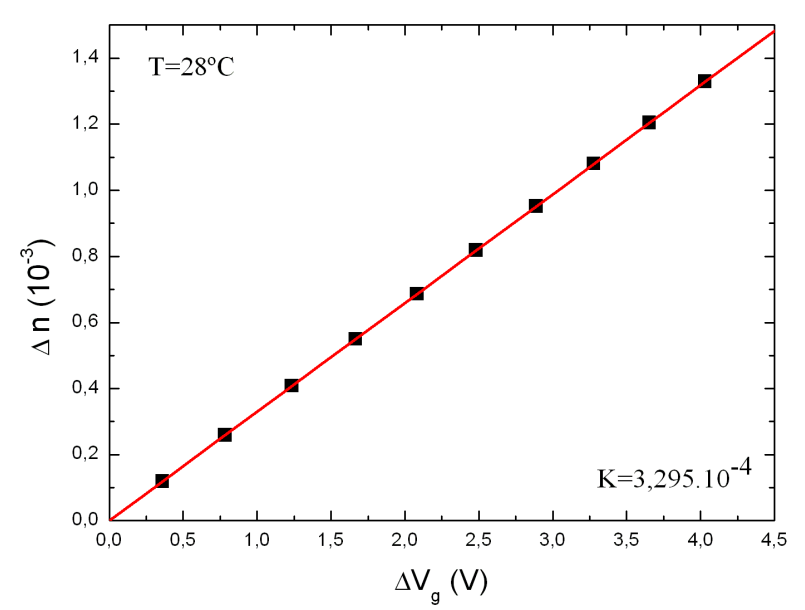

Figura 5.14: Curva de calibração: Incremento do índice de refração com relação à concentração, para o $K C l$ a $28^{\circ} C$.

\subsubsection{Incremento no índice de refração do DMPG}

A seguir apresentamos os resultados obtidos para o incremento do índice de refração para o DMPG.

$\mathrm{Na}$ fase intermediária, o incremento no índice de refração, para o DMPG, a $T=$ $20^{\circ} \mathrm{C}$ pode ser obtido por meio gráfico dado na figura 5.16, e corresponde a $d n / d c=$ $0,142 \mathrm{~mL} / \mathrm{g}$.

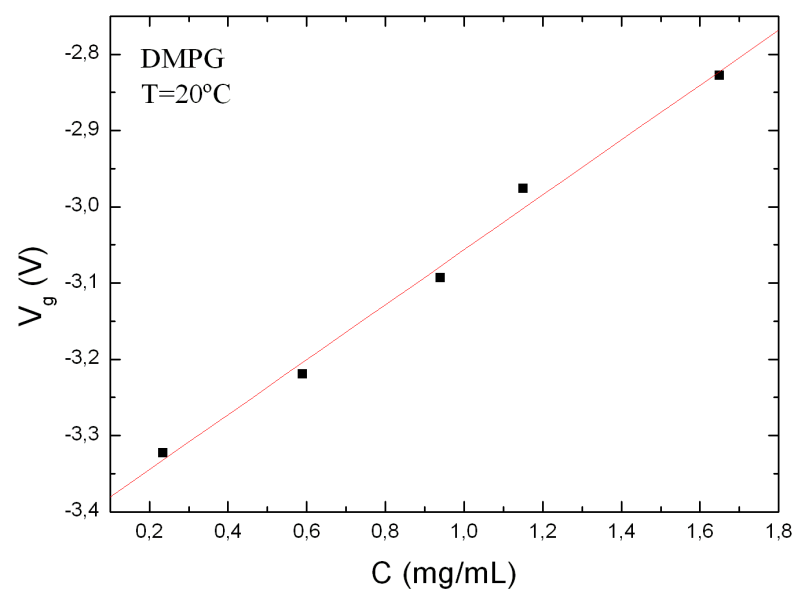

Figura 5.15: Ganho na voltagem com relação à concentração, para o DMPG a $20^{\circ} \mathrm{C}$.

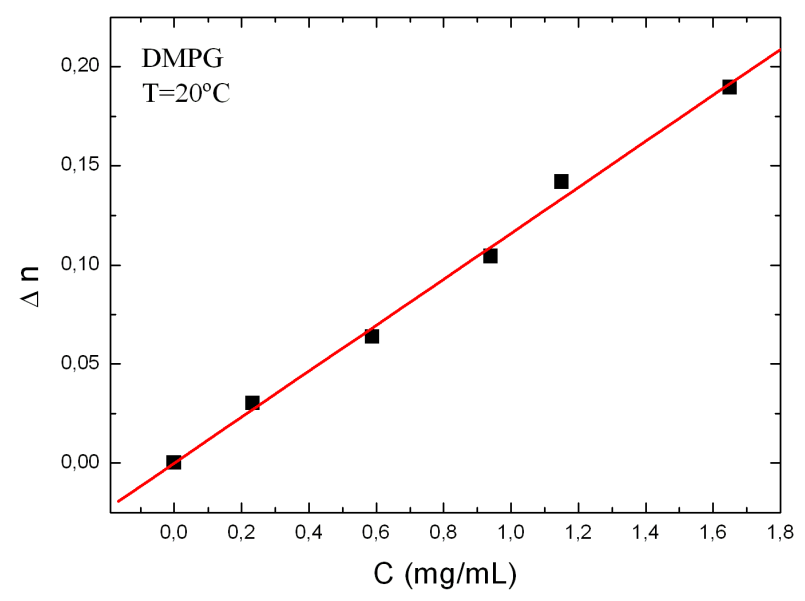

Figura 5.16: Incremento do índice de refração com relação à concentração, para o DMPG a $20^{\circ} C$.

Para $T=28^{\circ} \mathrm{C}$, temos $d n / d c=0,136 \mathrm{~mL} / \mathrm{g}$, e os resultados são apresentados nas figuras seguintes, 5.17 e 5.18 .

$\mathrm{O}$ incremento do índice de refração para o DMPG, na fase fluida à $T=43^{\circ} \mathrm{C}$ medido 


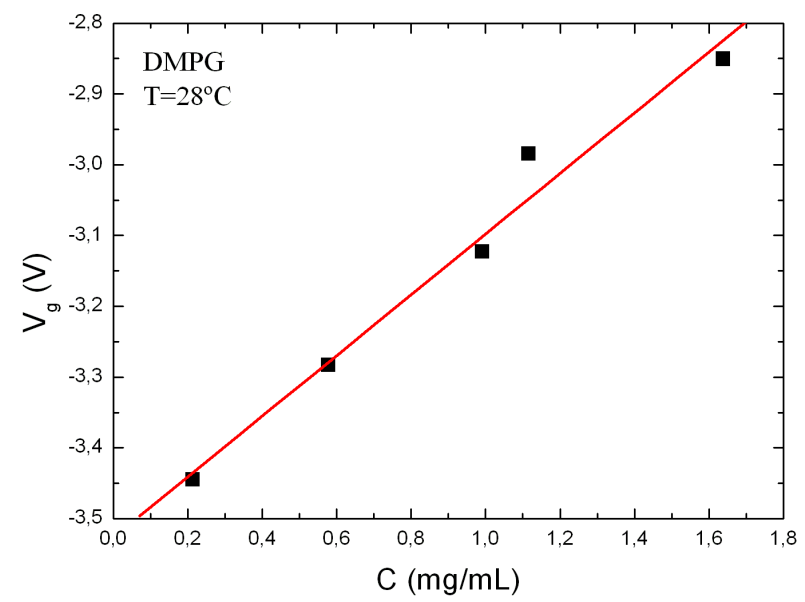

Figura 5.17: Ganho na voltagem com relação à concentração, para o DMPG a $28^{\circ} \mathrm{C}$.

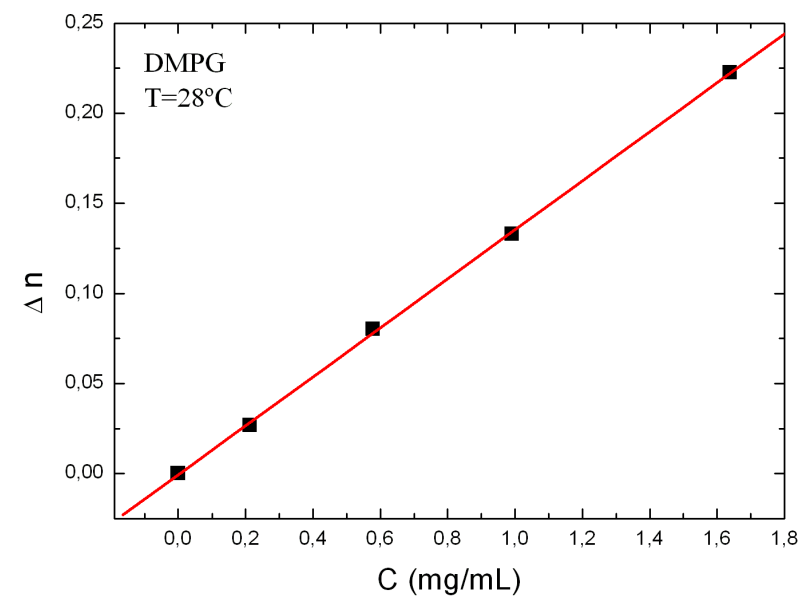

Figura 5.18: Incremento do índice de refração com relação à concentração, para o $\mathrm{DMPG}$ a $28^{\circ} \mathrm{C}$.

responde pelo valor $d n / d c=0,117 \mathrm{~mL} / \mathrm{g}$. Os resultados para esta temperatura foram utilizados como exemplos anteriormente e são dados pelas figuras 5.9 e 5.10.

O índice de refração, ou mesmo o incremento no índice de refração são dependentes da temperatura, admitindo que a dependência com a temperatura seja linear no intervalo analisado. O valor de $d n / d c$ para o DMPG na fase gel, $T=16^{\circ} C$, foi estimado, ${ }^{1}$ através do gráfico 5.19 .

Os valores obtidos de $d n / d c$ para o DMPG estão resumidos na tabela 5.3.

Tabela 5.3: Incremento no índice de refração para o DMPG, na fase gel, na região de transição e na fase fluída.

\begin{tabular}{|c||c|}
\hline Temperatura $\left({ }^{\circ} \mathrm{C}\right)$ & $d n / d c(\mathrm{~mL} / \mathrm{g})$ \\
\hline \hline 16 & 0,146 \\
\hline 20 & 0,142 \\
\hline 28 & 0,136 \\
\hline 43 & 0,117 \\
\hline
\end{tabular}

${ }^{1}$ Não é possível fazer medidas no refratômetro diferencial utilizado, em temperaturas abaixo da temperatura ambiente. 


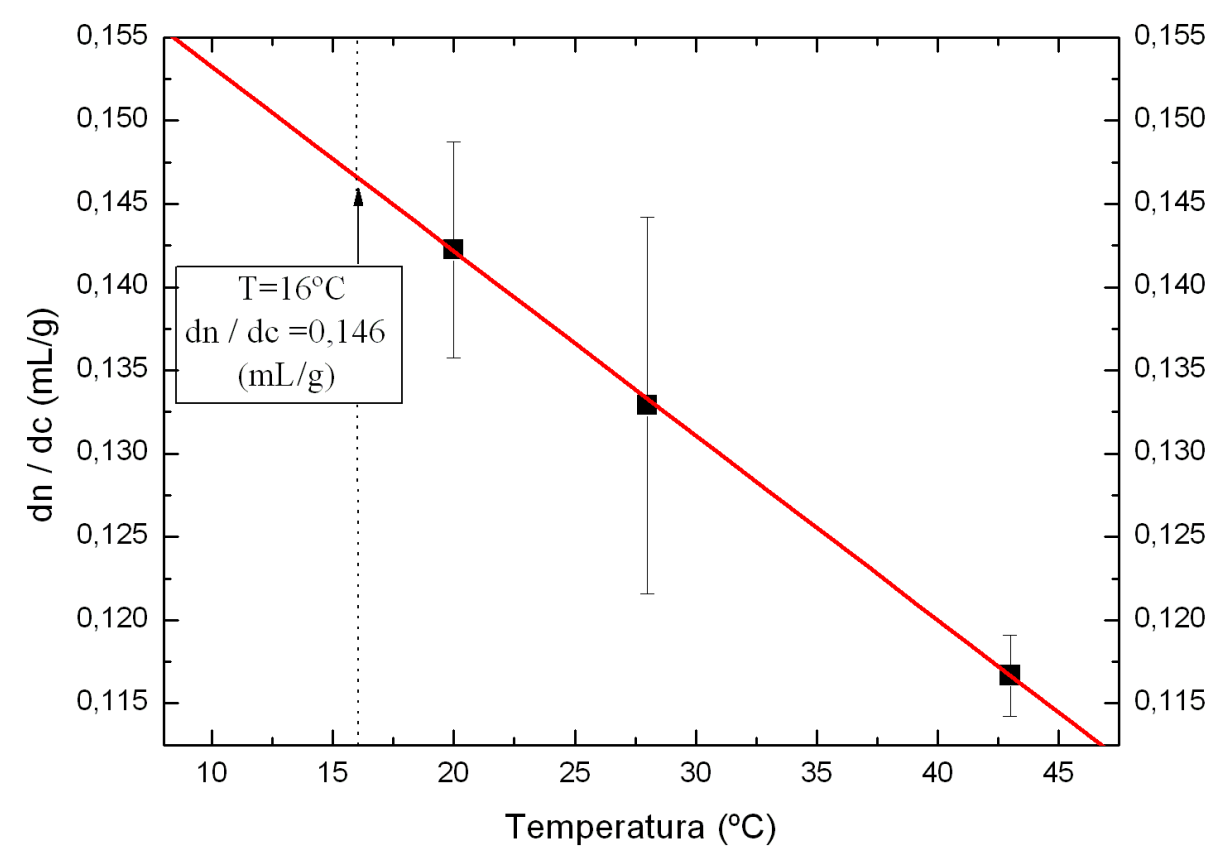

Figura 5.19: Incremento do índice de refração, para o DMPG em $T=16^{\circ} C$, estimado pela regressão linear dos dados experimentais.

\subsubsection{Incremento no índice de refração do DMPC}

Para o DMPC medimos o incremento no índice de refração na fase gel $T=20^{\circ} \mathrm{C}$ e na fase fluída $T=43^{\circ} \mathrm{C}$.

Na fase gel $T=20^{\circ} C$, encontramos o seguinte valor $d n / d c=0,155 \mathrm{~mL} / \mathrm{g}$, e os resultados são dados a seguir.

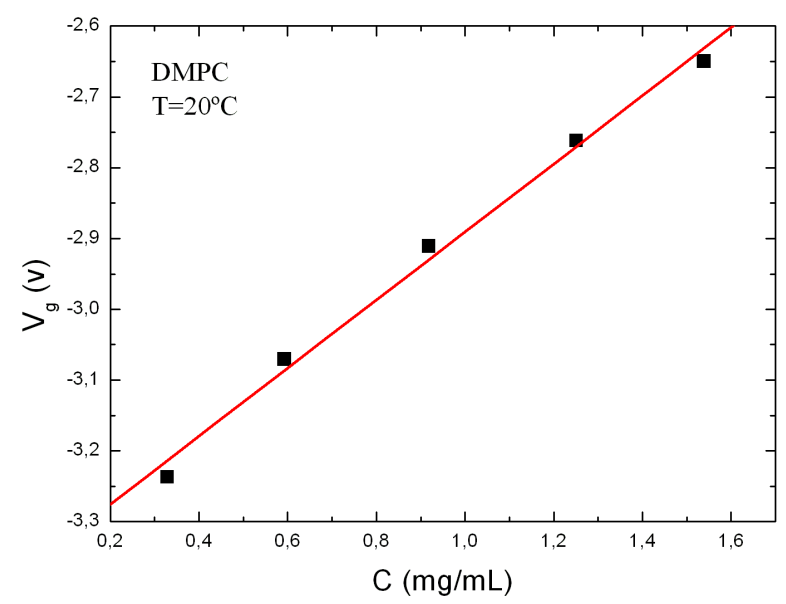

Figura 5.20: Ganho na voltagem com relação à concentração, para o DMPC a $20^{\circ} \mathrm{C}$.

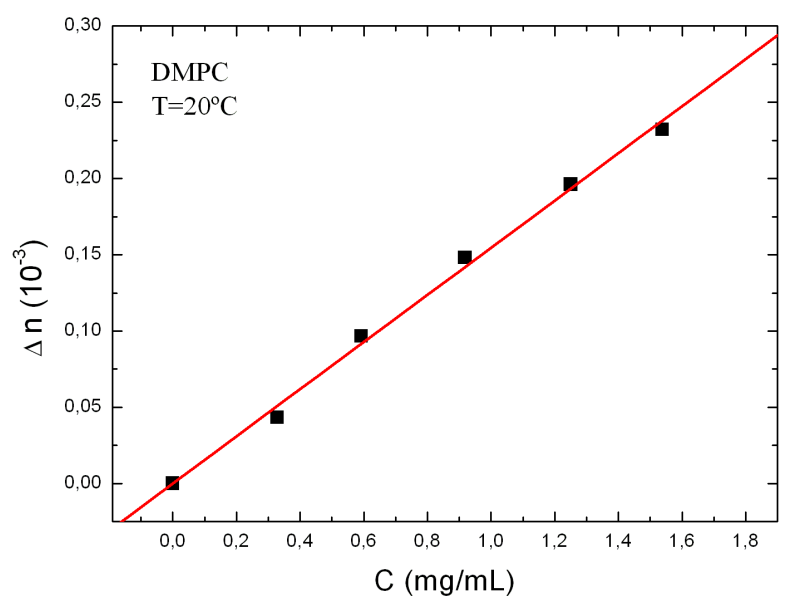

Figura 5.21: Incremento do índice de refração com relação à concentração, para o DMPC a $20^{\circ} \mathrm{C}$. 
Na fase fluída $T=43^{\circ} \mathrm{C}$, o valor medido corresponde a $d n / d c=0,121 \mathrm{~mL} / \mathrm{g}$, e os resultados são dados pelos gráficos 5.22 e 5.23.

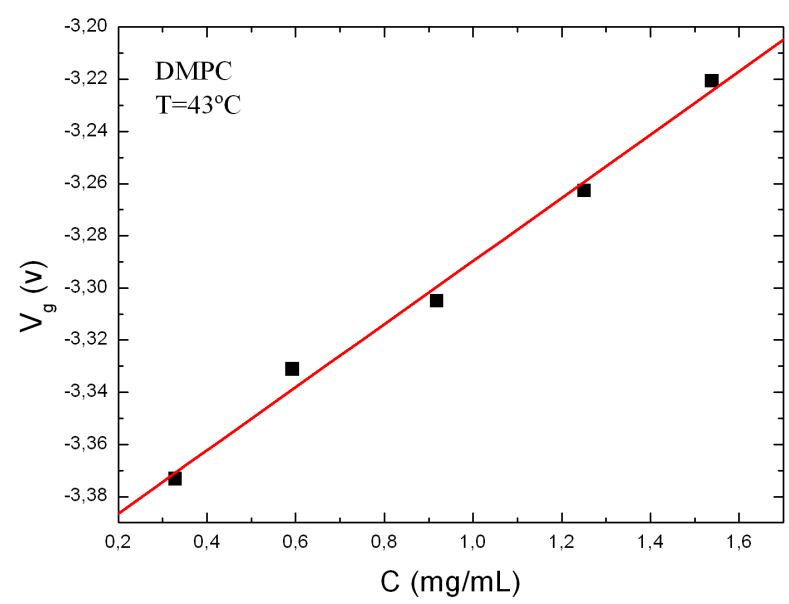

Figura 5.22: Ganho na voltagem com relação à concentração, para o DMPC a $43^{\circ} \mathrm{C}$.

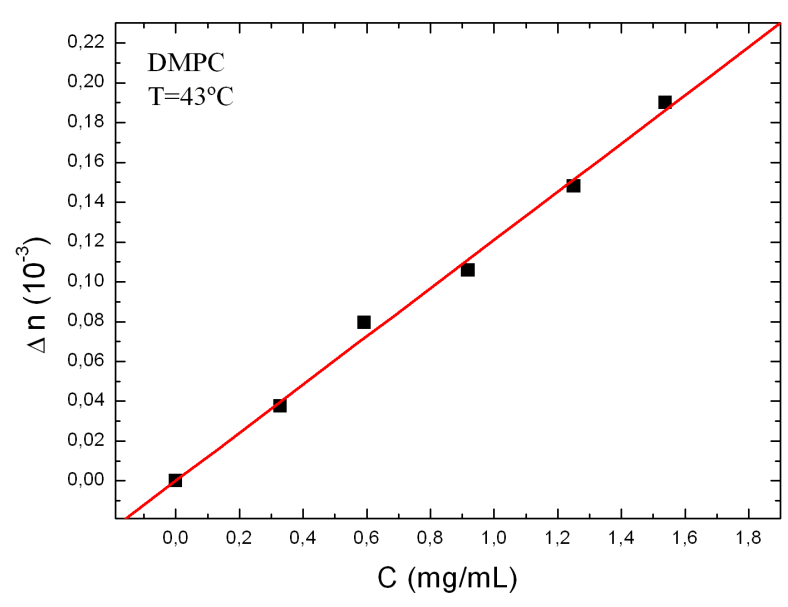

Figura 5.23: Incremento do índice de refração com relação à concentração, para o DMPC a $43^{\circ} \mathrm{C}$.

Na temperatura de transição de fase do DMPC como mostra a figura 5.24.

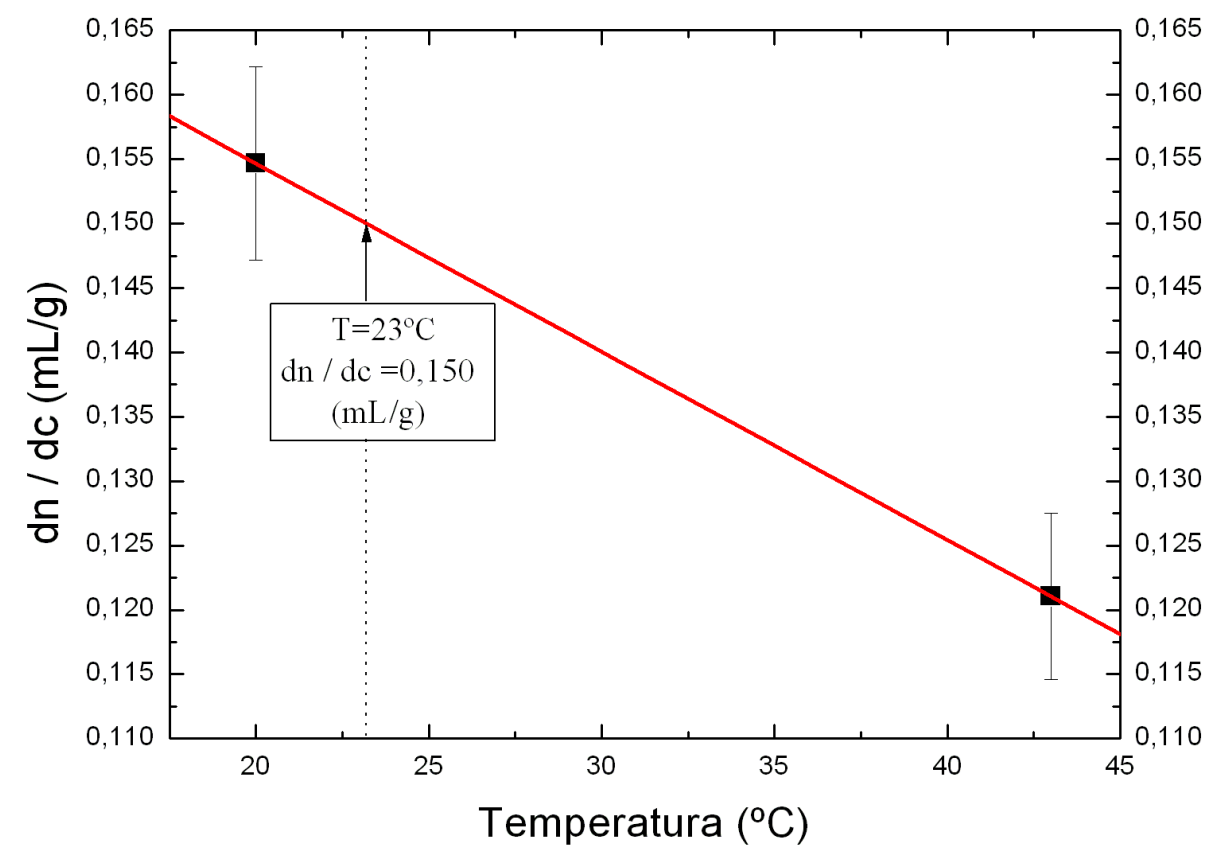

Figura 5.24: Incremento do índice de refração, para o DMPC em $T=23^{\circ} C$, estimado pela regressão linear dos dados experimentais. 
Os valores de $d n / d c$ para o DMPC, estão resumidos na tabela 5.4.

Tabela 5.4: Incremento no índice de refração para o DMPC, na fase gel, na transição de fase e na fase fluída.

\begin{tabular}{|c||c|}
\hline Temperatura $\left({ }^{\circ} \mathrm{C}\right)$ & $d n / d c(\mathrm{~mL} / \mathrm{g})$ \\
\hline \hline 20 & 0,155 \\
\hline 23 & 0,150 \\
\hline 43 & 0,121 \\
\hline \hline
\end{tabular}

Os valores de $d n / d c$ obtidos nas tabelas 5.3 e 5.4 serão utilizados nas análises de Zimm. 


\subsection{Calorimetria Diferencial de Varredura}

A técnica de calorimetria mede a razão entre a quantidade de calor fornecida a um sistema fechado à pressão constante e a consequente variação na temperatura. Esta é a própria definição de capacidade térmica, (Salinas, 2008).

$$
C p=\left.\lim _{\Delta T \rightarrow 0} \frac{\Delta Q}{\Delta T}\right|_{p, V}
$$

Para avaliarmos a capacidade térmica do soluto (vesículas de DMPG e DMPC), devemos subtrair a quantidade de calor fornecida ao solvente.

O calorímetro diferencial de varredura (DSC) utiliza um sistema que contém duas celas. Uma das celas porta apenas o solvente, chamada de cela da referência, e a outra cela porta a solução (solvente + soluto), nomeada de cela da amostra. Durante a transição de fase a cela da amostra absorve mais calor. A potência dos aquecedores (da cela da amostra e da cela da referência) é finamente ajustada para que a taxa de variação de temperatura por unidade de tempo, $\left({ }^{\circ} \mathrm{C} / \mathrm{s}\right)$, denominada scan rate seja preservada constante. Em outras palavras ambas as celas apresentam a mesma varição de temperatura por unidade de tempo. Além disso, as duas celas são sempre mantidas a mesma temperatura, (Heimburg, 2007).

A diferença das potências dos aquecedores (das celas da amostra e da referência) é então registrada em função da temperatura. Essa diferença de potência (Cal/s), é dividida pelo scan rate $\left({ }^{\circ} \mathrm{C} / \mathrm{s}\right)$, fornecendo portanto a Capacidade térmica do soluto, $C_{p}$.

A diferença de potência vista na cela da amostra com relação à cela da referência ocorre porque a cela da amostra precisa de uma quantidade de calor $\Delta Q^{\prime}$ diferente da cela da referência, $\Delta Q$, para elevar o sistema (amostra ou referência) ao mesmo incremento de temperatura, $\Delta T$, (scan rate constante). A quantidade de calor fornecida a amostra é maior que a quantidade de calor fornecida à referência $\Delta Q^{\prime}>\Delta Q$, pois parte do calor fornecido à amostra, durante a transição é destinado a mudanças microscópicas do soluto. No caso das vesículas de DMPG e DMPC essa quantidade de calor aumenta a desordem das cadeias carbônicas, por exemplo, como podemos observar em um esquema ilustrativo na figura 5.25 . 


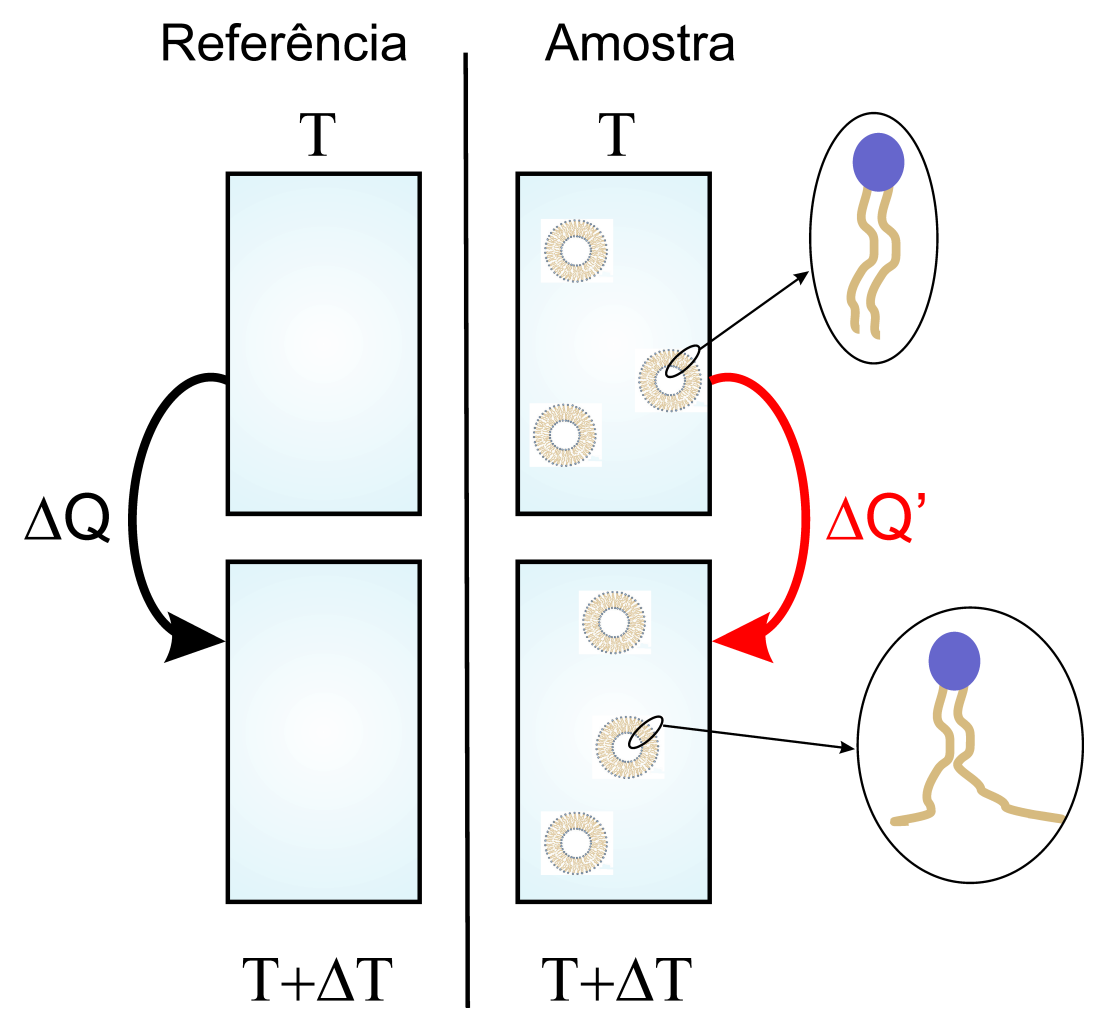

Figura 5.25: Ilustração da medida de calorimetria.

Do ponto de vista microscópico, moléculas com o mesmo tipo de agregação, em um sistema homogêneo, compõem uma fase.

$\mathrm{Na}$ fase gel os lipídios apresentam um determinado empacotamento, as cabeças polares dos lipídios são arranjadas em uma rede triangular (Janiak et al., 1979), e suas cadeias laterais assumem conformações estendidas e altamente ordenadas. Contudo, na fase fluida as cabeças polares não se organizam em uma rede ordenada e suas cadeias hidrofóbicas apresentam configurações aleatórias, como vimos na figura 1.1. A entropia do sistema ordenado diferente muito da entropia do sistema desordenado. Essa diferença brusca de entropia pode caracterizar uma transição de fase.

Visto que $\Delta Q=T \Delta S$, então, a equação 5.6, pode ser escrita sob a forma

$$
C p=\left.\lim _{\Delta T \rightarrow 0} T \frac{\Delta S}{\Delta T}\right|_{p, V}=\left.T\left(\frac{\partial S}{\partial T}\right)\right|_{p, N} .
$$

Por isso a brusca diferença de entropia dada por uma transição ordem-desordem pode ser evidenciada como um pico na capacidade térmica.

De acordo com a Primeira Lei da Termodinâmica

$$
d U=d Q+d W
$$

onde $d W$ corresponde ao trabalho fornecido ao sistema. Também podemos escrever

$$
d U=T d S-p d V
$$


e através de uma transformada de Legendre para a energia $\mathrm{U}(\mathrm{S}, \mathrm{V}, \mathrm{N})$, podemos obter o potencial termodinâmico conhecido por Entalpia

$$
H(S, p, V)=U+p V
$$

portanto

$$
\begin{aligned}
& d H=d U+p d V+V d p \\
& d H=T d S+V d p,
\end{aligned}
$$

à pressão constante

$$
d H=T d S
$$

Logo a equação 5.7, também pode ser escrita na forma

$$
C p=\left.\left(\frac{\partial H}{\partial T}\right)\right|_{p, N} .
$$

Portanto, a integral sob a curva de Cp, em função da temperatura, corresponde à entalpia envolvida na transição de fase.

Os resultados de Calorimetria, acompanhados de discussões serão apresentados no capítulo 6. 



\section{Capítulo 6}

\section{Resultados e discussões}

\subsection{Calorimetria Diferencial de Varredura}

Este trabalho foi desenvolvido com dispersões de DMPG extrusadas, pois a polidispersão dificulta a caracterização das amostras por espalhamento de luz. É também sabido que dispersões de DMPC formam vesículas multilamelares de tamanhos variados e muito grandes, (Riske et al., 2004). Deste modo, foram realizados experimentos de calorimetria diferencial de varredura, DSC, para verificar se o comportamento peculiar termo-estrutural do DMPG era preservado em dispersões extrusadas.

Os resultados de DSC para o DMPG extrusado evidenciam o mesmo perfil das curvas de capacidade térmica do DMPG não extrusado, apresentando uma região de transição de fase, entre a fase gel e a fase fluida, conforme podemos observar na figura 6.1, para três diferentes amostras. 


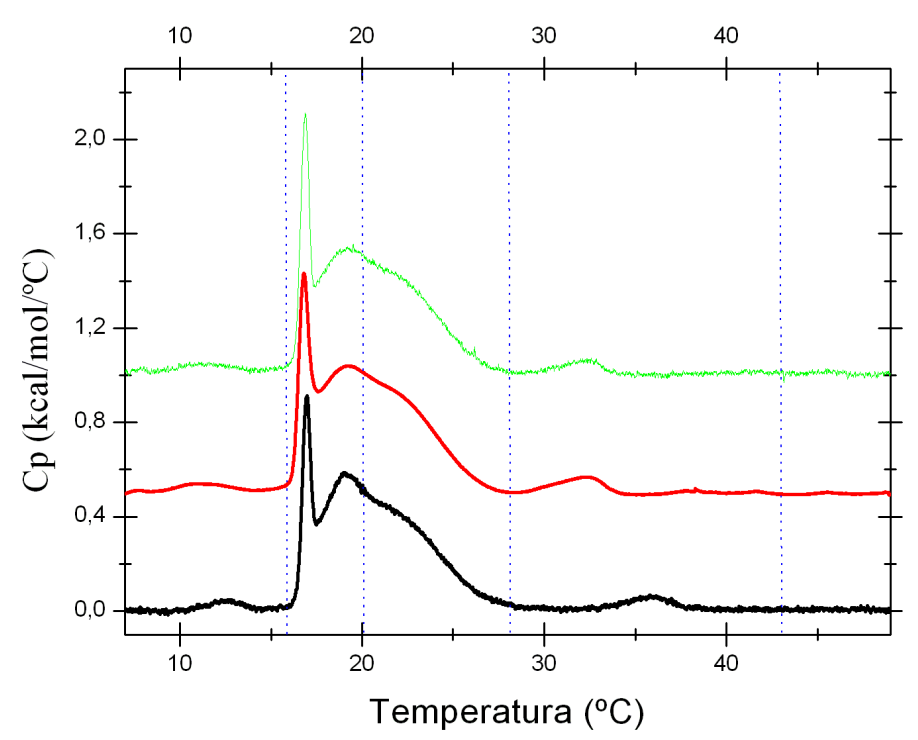

Figura 6.1: Capacidade térmica em função da temperatura para o DMPG extrusado. As medidas de calorimetria foram realizadas com scan rate $20^{\circ} / \mathrm{h}$. As linhas pontilhadas ilustram as temperaturas em que realizamos as medidas de espalhamento de luz, SLS e DLS. As temperaturas são: $T=16^{\circ} \mathrm{C}$ (fase gel), $T=20$ e $28^{\circ} \mathrm{C}$ (região de transição) e $T=43^{\circ} \mathrm{C}$ (fase fluida). As curvas de DSC foram deslocadas na vertical para facilitar a visualização.

Podemos verificar algumas mudanças com relação aos traços de DSC para as dispersões lipídicas extrusadas e não extrusadas, observe a figura 6.2. A principal delas é a diminuição do primeiro pico estreito, em $T_{m}^{o n}$, mostrando a diminuição desse evento térmico, mais cooperativo.

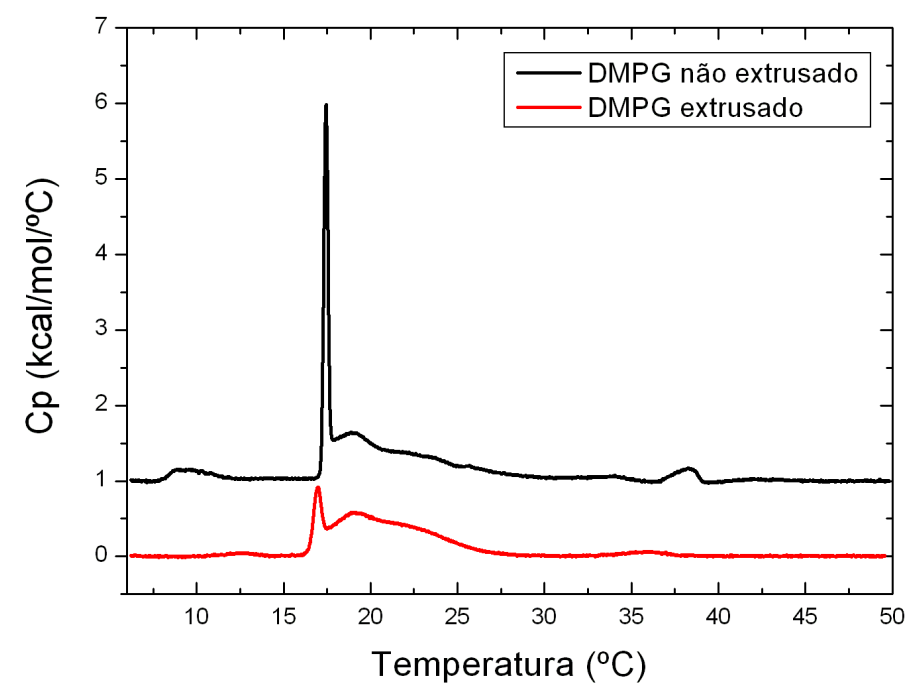

Figura 6.2: Capacidade térmica em função da temperatura para o DMPG extrusado e não extrusado. As medidas de calorimetria foram realizadas com scan rate $20^{\circ} / h$. O gráfico ilustra três amostras distintas que foram preparadas nas mesmas condições. As curvas de DSC foram deslocadas na vertical para facilitar a visualização. 
As curvas de capacidade térmica para o DMPC também diferem quanto a diminuição e alargamento do pico da transição principal, observe a figura 6.3.
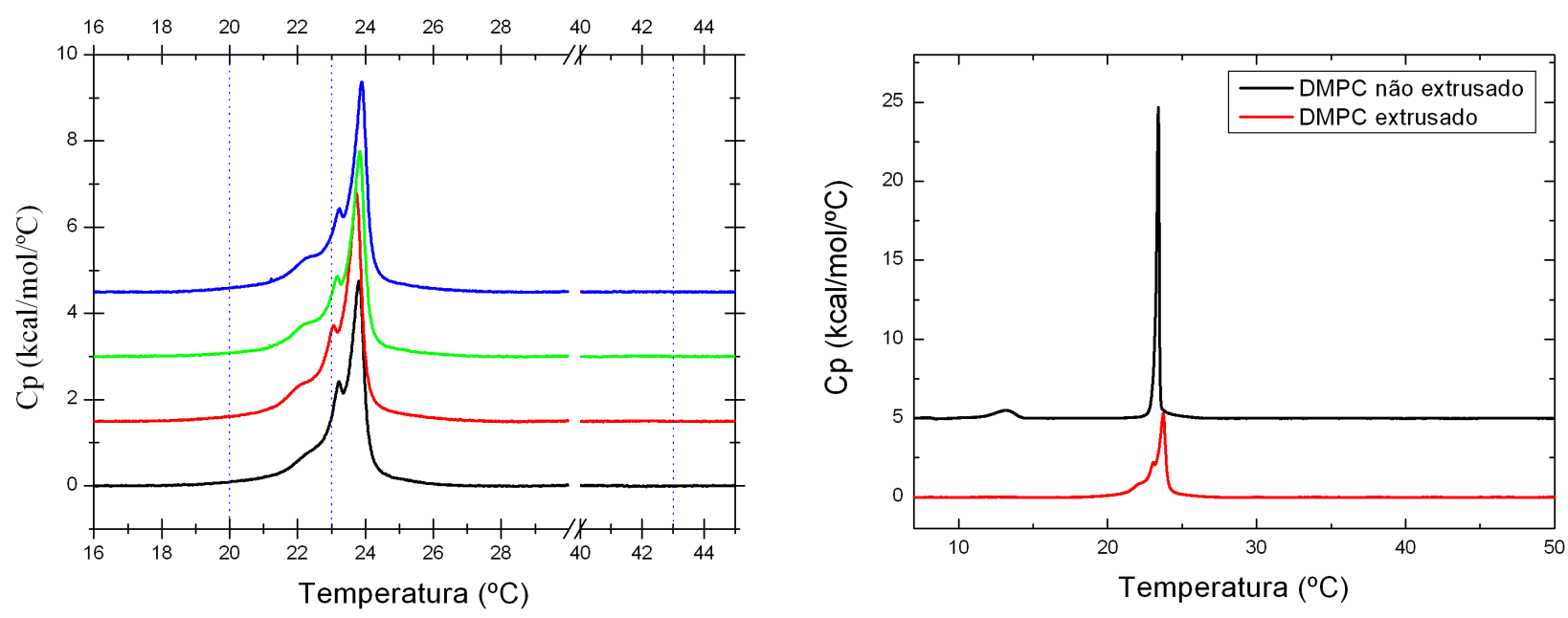

Figura 6.3: Capacidade térmica em função da temperatura para o DMPC extrusado e não extrusado. As medidas de calorimetria foram realizadas com scan rate $20^{\circ} / \mathrm{h}$. As linhas pontilhadas do gráfico da esquerda ilustram as temperaturas em que realizamos as medidas de espalhamento do luz, SLD e DLS. As temperaturas são: $T=20^{\circ} C$ (fase gel), $T=23$ (transição de fase ) e $T=43^{\circ} \mathrm{C}$ (fase fluida). As curvas de DSC foram deslocadas na horizontal para facilitar a visualização.

Os resultados de calorimetria para os lipídios não extrusados indicam um alto efeito cooperativo. Quando os agregados lipídicos são extrusados por um filtro de $100 \mathrm{~nm}$, em média, eles assumem tamanhos menores aumentando assim a curvatura da bicamada.

Podemos avaliar a influência da curvatura das vesículas em relação a seu comportamento termo estrutural (aumentando a temperatura). Vesículas multilamelares como o caso do DMPC não extrusado, são tipicamente grandes e suas bicamadas são consideradas praticamente planas. Deste modo, durante a transição de fase a probabilidade de encontrar um lipídio na fase fluida na camada superior é a mesma para a camada inferior (Heimburg, 2007).

No entanto quanto maior a curvatura da bicamada maior a probabilidade de encontrar um lipídio na fase fluida na camada superior. Isto porque a área ocupada por cabeça polar de lipídio na camada externa passa a ser maior que na camada interna, favorecendo primeiro a transição da camada externa e diminuindo a cooperatividade, (Heimburg, 2007).

Sabemos que a curvatura das vesículas aumentou com relação as dispersões não extrusadas, pois acompanhamos os valores dos diâmetros efetivos, medindo as dispersões lipídicas por DLS. Fizemos um estudo cuidadoso variando o número de extrusões. Obser- 
vamos para o DMPC, que as dispersões não extrusadas apresentavam tamanhos grandes da ordem de $\sim \mu m$, já era sabido que o DMPC forma vesículas multilamelares grandes. Quando extrusávamos, essas dispersões, 11 vezes por um filtro de $100 \mathrm{~nm}$, obtínhamos tamanhos da ordem de $120 \mathrm{~nm}$, e quando extrusavámos 31 vezes obtínhamos diâmetros da ordem de $\sim 100 \mathrm{~nm}$. Supomos que no segundo caso a variação de tamanhos para as dispersões de DMPC fossem menores. Por isso adotamos como padrão extrusar todas as dispersões 31 vezes.

No entanto para o DMPG obtivemos um resultado surpreendente. O DMPG não extrusado apresentava um valor da ordem de $120 \mathrm{~nm}$ e quando extrusávamos a dispersão por um filtro de $100 \mathrm{~nm}$ obtínhamos tamanhos da ordem de $50 \mathrm{~nm}$. Isso pode indicar que o DMPG é composto por uma grande população de tamanhos pequenos. Os resultados para o DMPG podem ser vistos com mais detalhe no apêndice C. 


\subsection{Espalhamento de Luz Estático}

Os resultados de espalhamento de luz estático foram obtidos e analisados por meio do método de Zimm, conforme a equação (2.111),

$$
\frac{K c}{\Delta R_{\theta}}=\left\{1+\frac{R_{g}^{2} q^{2}}{3}\right\}\left\{\frac{1}{M}+2 A_{2} c\right\} .
$$

O primeiro termo entre $\{\cdots\}$ consiste na expansão do fator de forma, enquanto que o segundo termo refere-se a expansão no fator de estrutura, conforme vimos no Capítulo 2. Na análise de Zimm são estabelecidos dois limites. Um dos limites refere-se ao fator de forma tendendo a zero, $q \rightarrow 0$, e a intensidade da luz espalhada torna-se uma resposta do fator de estrutura. O outro limite corresponde ao fator de estrutura tendendo a zero, $c \rightarrow 0$, para estudo do fator de forma.

Para a dispersão lipídica de DMPG, avaliamos quatro temperaturas em que os lipídios estão em diferentes fases. Em $T=16^{\circ} \mathrm{C}$, os lipídios encontram-se na fase gel, em $T=20^{\circ} \mathrm{C}$ e $T=28^{\circ} \mathrm{C}$ temos a região de transição, e em $T=43^{\circ} \mathrm{C}$, temos a fase fluida. Em particular, na temperatura $T=28^{\circ} \mathrm{C}$ encontramos na literatura um valor máximo para a viscosidade e a condutividade, (Barroso et al., 2010).

Para os gráficos a seguir, referentes ao DMPG, utilizamos os valores de $d n / d c$ dados pela tabela 5.3 .
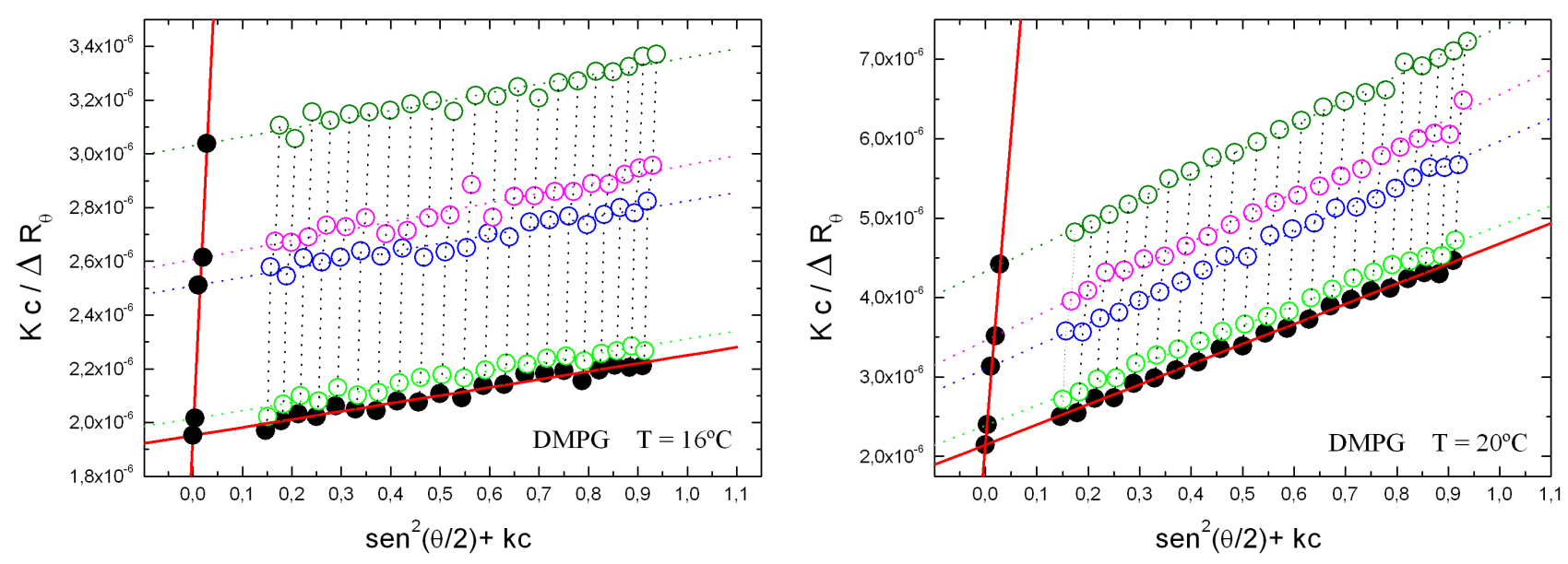

Gráficos de Zimm para o DMPG $(C=0,214 ; 0,579 ; 1,160$ e $1,640 \mathrm{mg} / \mathrm{mL})$, em diferentes temperaturas - (grupo $1)$. 

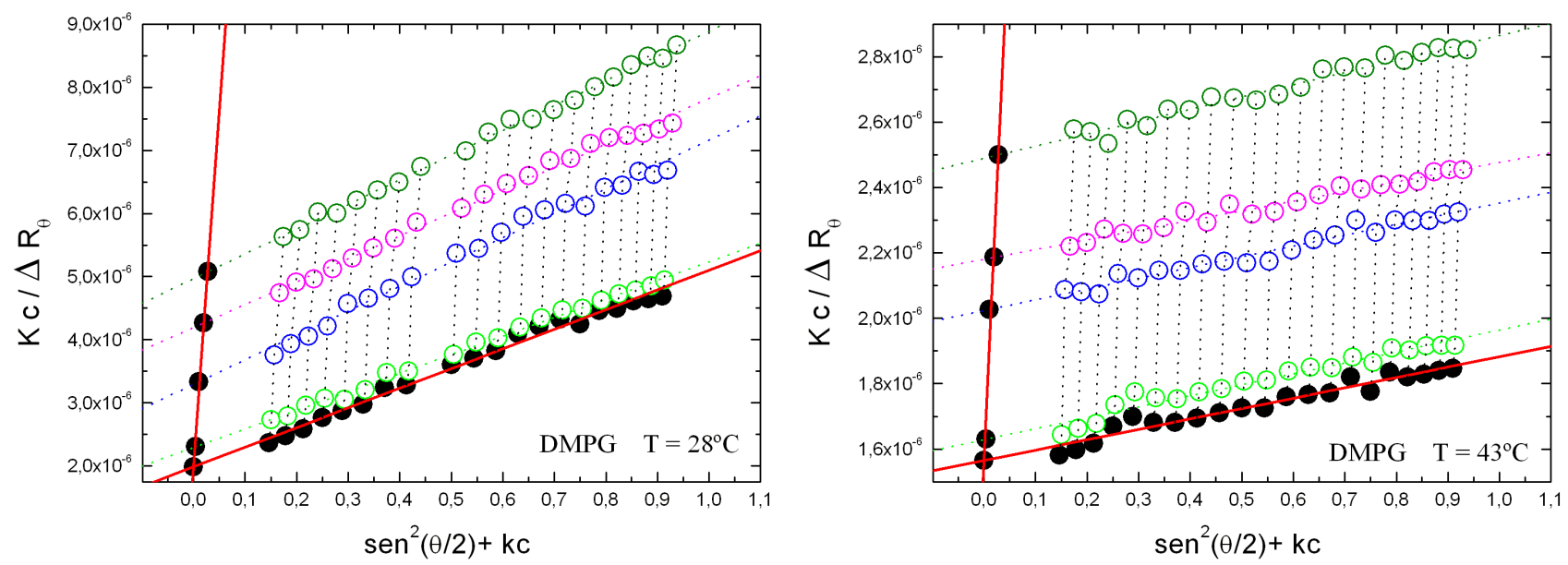

Figura 6.4: Gráficos de Zimm para o DMPG $(C=0,214 ; 0,579 ; 1,160$ e 1,640 $\mathrm{mg} / \mathrm{mL})$, em diferentes temperaturas - (grupo 1).

As concentrações utilizadas nas análises da figura 6.4 são dadas pela tabela 5.1 e referem-se à figura 5.2. Os valores obtidos para $\bar{M}_{w}, R_{g}$ e $A_{2}$ estão resumidos na tabela 6.1

Tabela 6.1: Resultados dos gráficos de Zimm para o DMPG $(C=0,214 ; 0,579 ; 1,160$ e $1,640 \mathrm{mg} / \mathrm{mL})$, em diferentes temperaturas - (grupo 1).

\begin{tabular}{|cccc|}
\hline Temperatura ${ }^{\circ} \mathrm{C}$ & $\bar{M}_{w}\left(10^{5} \mathrm{~g} / \mathrm{mol}\right)$ & $R_{g}(\mathrm{~nm})$ & $A_{2}\left(10^{-4} \mathrm{~cm}^{3} \mathrm{~mol} / \mathrm{g}^{2}\right)$ \\
\hline \hline 16 & $5,1 \pm 0,4$ & $25,6 \pm 0,7$ & $3,2 \pm 0,7$ \\
20 & $4,7 \pm 0,5$ & $71,2 \pm 0,8$ & $6,6 \pm 0,9$ \\
28 & $5,0 \pm 0,1$ & $82 \pm 2$ & $9,5 \pm 0,9$ \\
43 & $6,4 \pm 0,4$ & $29,5 \pm 0,9$ & $2,8 \pm 0,5$ \\
\hline \hline
\end{tabular}

Repetimos a análise de Zimm com um conjunto diferente de amostras, cujas concentrações são dadas pela tabela 5.1 referente à figura 5.3. Os resultados estão dispostos na figura 6.5 e na tabela 6.2 .

Tabela 6.2: Resultados dos gráficos de Zimm para o DMPG $(C=0,255 ; 0,889 ; 1,185$ e $1,660 \mathrm{mg} / \mathrm{mL})$, em diferentes temperaturas - (grupo 2).

\begin{tabular}{|cccc|}
\hline Temperatura ${ }^{\circ} \mathrm{C}$ & $\bar{M}_{w}\left(10^{5} \mathrm{~g} / \mathrm{mol}\right)$ & $R_{g}(\mathrm{~nm})$ & $A_{2}\left(10^{-4} \mathrm{~cm}^{3} \mathrm{~mol} / \mathrm{g}^{2}\right)$ \\
\hline \hline 16 & $4,3 \pm 0,4$ & $30,7 \pm 0,7$ & $5,3 \pm 1,2$ \\
20 & $3,6 \pm 0,6$ & $83 \pm 2$ & $10 \pm 2$ \\
28 & $3,4 \pm 0,1$ & $92 \pm 1$ & $13,9 \pm 0,5$ \\
43 & $5,8 \pm 0,6$ & $35,5 \pm 0,6$ & $4,2 \pm 0,9$ \\
\hline \hline
\end{tabular}



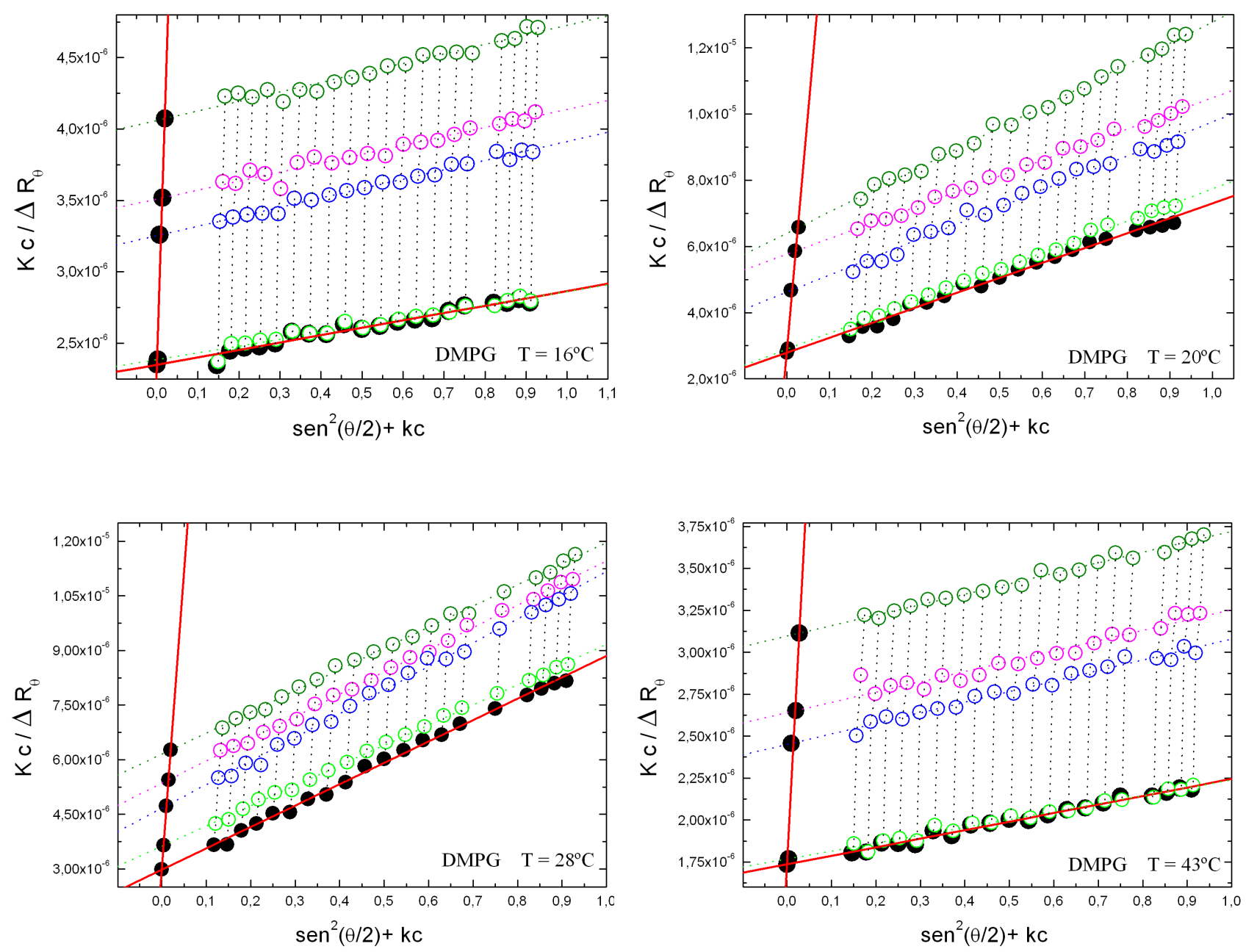

Figura 6.5: Gráficos de Zimm para o DMPG $(C=0,255 ; 0,889 ; 1,185$ e 1,660 $\mathrm{mg} / \mathrm{mL})$, em diferentes temperaturas - (grupo 2).

Optamos por representar a reprodutibilidade das medidas mostrando as análises dos dois grupos de amostras para o mesmo sistema. Identificamos as analises de Zimm para o DMPG nos grupos: grupo 1 dado pela figura 6.4 e tabela 6.1, e grupo 2 dado pela figura 6.5 e tabela 6.2 .

Os gráficos de Zimm apresentaram ótimos ajustes para o DMPG, com coeficientes de determinação ${ }^{1} R$ superior a 0,96 , poucos pontos foram descartados, e apenas uma concentração, nos dois grupos de amostras, se diferenciou das demais e não foi utilizada nestas análises. É importante enfatizar que na região de transição também obtivemos ótimos ajustes. Portanto, a aproximação linear (primeiro termo das expansões) para os fatores de forma e estrutura foram suficientes para ajustar os dados coletados, (conforme

\footnotetext{
${ }^{1} \mathrm{O}$ coeficiente de determinação refere-se à qualidade do ajuste, quanto mais próximo de uma unidade melhor o ajuste
} 
descrita no Capítulo 2).

Analisamos o DMPC na fase gel, $T=20^{\circ} \mathrm{C}$, na fase fluida, $T=43^{\circ} \mathrm{C}$, e na temperatura de transição de fase, $T=23^{\circ} \mathrm{C}$. Utilizamos os valores de $d n / d c$ apresentados na tabela 5.4. Tratamos igualmente dois grupos de amostras, e os resultados estão dispostos a seguir.

As análises da figura 6.6, foram realizadas utilizando as concentrações dosadas da tabela 5.2, referente a figura 5.5.
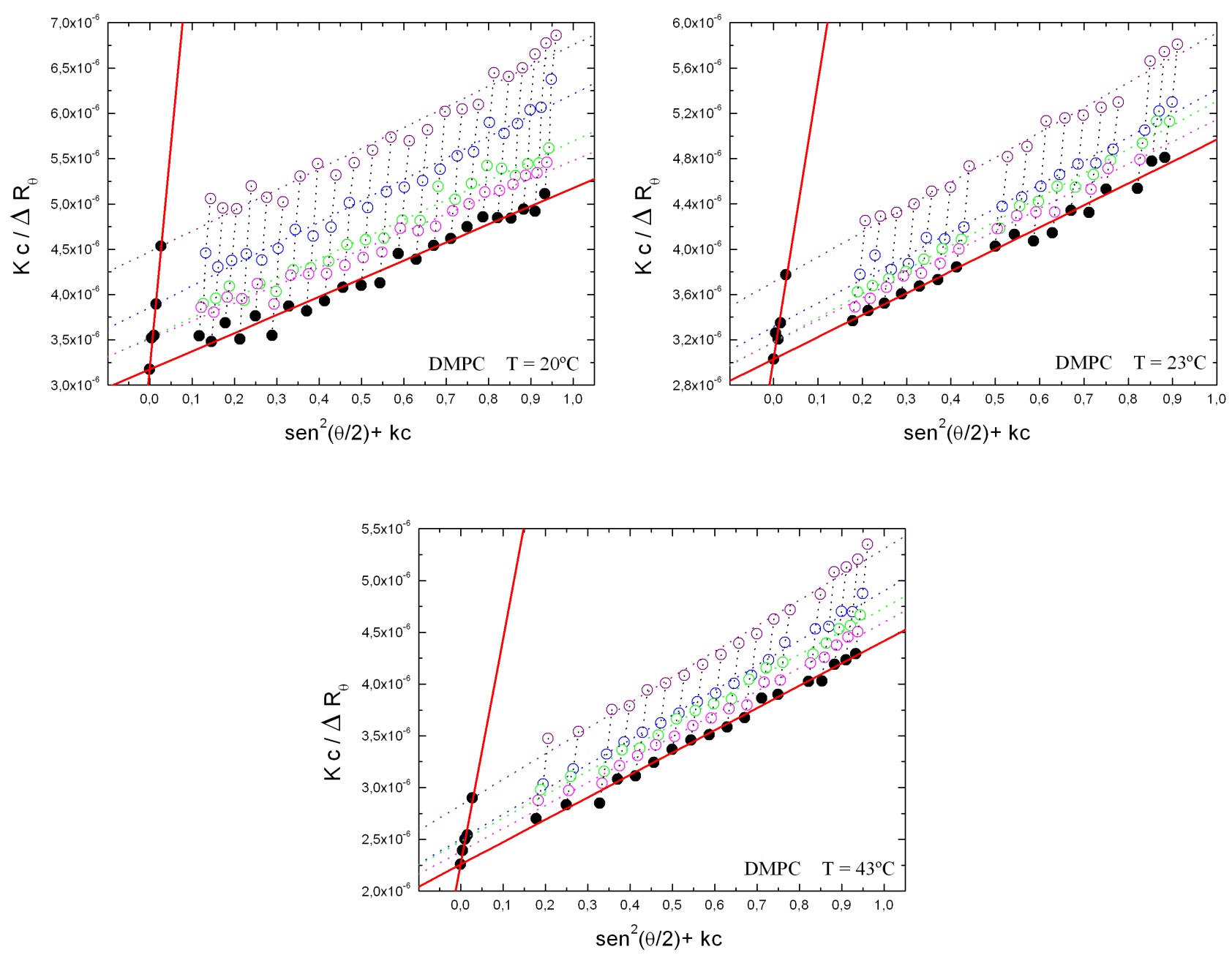

Figura 6.6: Gráficos de Zimm para o DMPC $(C=0,285 ; 0,590 ; 0,895$ e 1,566 mg/mL), em diferentes temperaturas - (grupo 1). 
Os valores de $\bar{M}_{w}, R_{g}$ e $A_{2}$ foram resumidos na tabela 6.3 .

Tabela 6.3: Resultados dos gráficos de Zimm para o DMPC $(C=0,285 ; 0,590 ; 0,895$ e $1,566 \mathrm{mg} / \mathrm{mL})$, em diferentes temperaturas - (grupo 1).

\begin{tabular}{|cccc|}
\hline Temperatura ${ }^{\circ} \mathrm{C}$ & $\bar{M}_{w}\left(10^{5} \mathrm{~g} / \mathrm{mol}\right)$ & $R_{g}(\mathrm{~nm})$ & $A_{2}\left(10^{-4} \mathrm{~cm}^{3} \mathrm{~mol} / \mathrm{g}\right)$ \\
\hline \hline 20 & $3,2 \pm 0,1$ & $52 \pm 1$ & $4,2 \pm 0,6$ \\
23 & $3,3 \pm 0,1$ & $52 \pm 1$ & $2,2 \pm 0,6$ \\
43 & $4,4 \pm 0,1$ & $64 \pm 1$ & $2,0 \pm 0,3$ \\
\hline \hline
\end{tabular}

Novamente as análises foram repetidas para um segundo grupo de amostras com concentrações dadas pela tabela 5.2, figura 5.4.
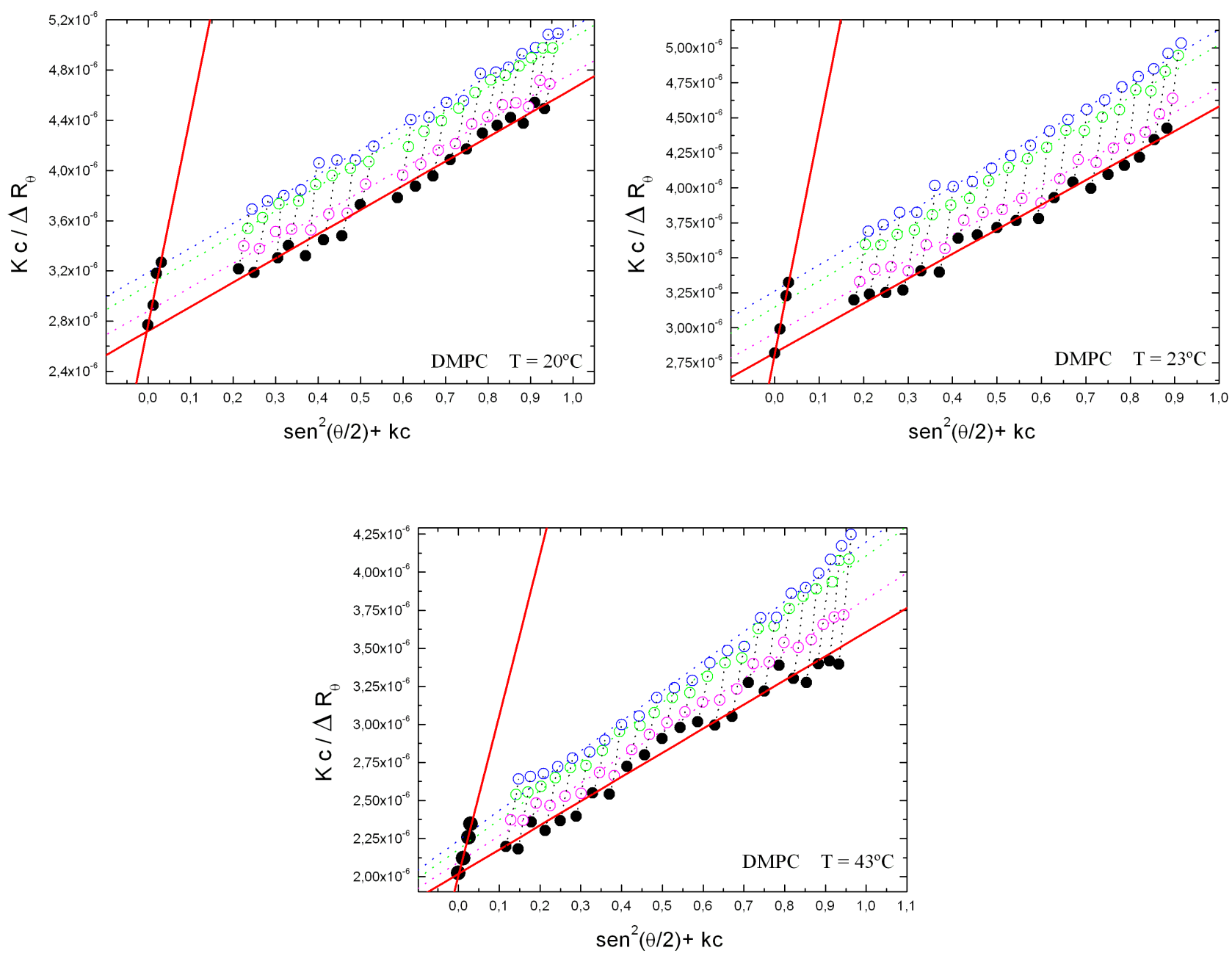

Figura 6.7: Gráficos de Zimm para o DMPC $(C=0,597 ; 0,942$ e 1,227 $\mathrm{mg} / \mathrm{mL})$, em diferentes temperaturas (grupo 2) . 
Os resultados das análises da figura 6.7 estão resumidos na tabela 6.4 .

Tabela 6.4: Resultados dos gráficos de Zimm para o DMPC $(C=0,597 ; 0,942$ e 1, $227 \mathrm{mg} / \mathrm{mL})$, em diferentes temperaturas - (grupo 2).

\begin{tabular}{|cccc|}
\hline Temperatura ${ }^{\circ} \mathrm{C}$ & $\bar{M}_{w}\left(10^{5} \mathrm{~g} / \mathrm{mol}\right)$ & $R_{g}(\mathrm{~nm})$ & $A_{2}\left(10^{-4} \mathrm{~cm}^{3} \mathrm{~mol} / \mathrm{g}^{2}\right)$ \\
\hline \hline 20 & $3,6 \pm 0,2$ & $54 \pm 2$ & $1,8 \pm 0,6$ \\
23 & $3,6 \pm 0,1$ & $52 \pm 2$ & $1,7 \pm 0,4$ \\
43 & $4,9 \pm 0,1$ & $58 \pm 2$ & $0,9 \pm 0,2$ \\
\hline \hline
\end{tabular}

Os ajustes obtidos para o DMPC foram um pouco inferiores aos ajustes obtidos para o DMPG, com coeficiente de determinação $R$ superior a 0,90. No segundo grupo de amostras, mostramos apenas três concentrações, pois duas das concentrações apresentavam-se sobrepostas.

Discutiremos apenas os valores obtidos para o $A_{2}$ e $R_{g}$, das análises de Zimm. Os dados de $\bar{M}_{w}$ são mais difíceis de serem interpretados para dispersões de vesícula lipídicas, onde a massa total é conservada (fora variações de hidratação e ionização).

Os resultados do segundo coeficiente do virial dos gráficos de Zimm podem ser resumidos no gráfico da figura 6.8 .

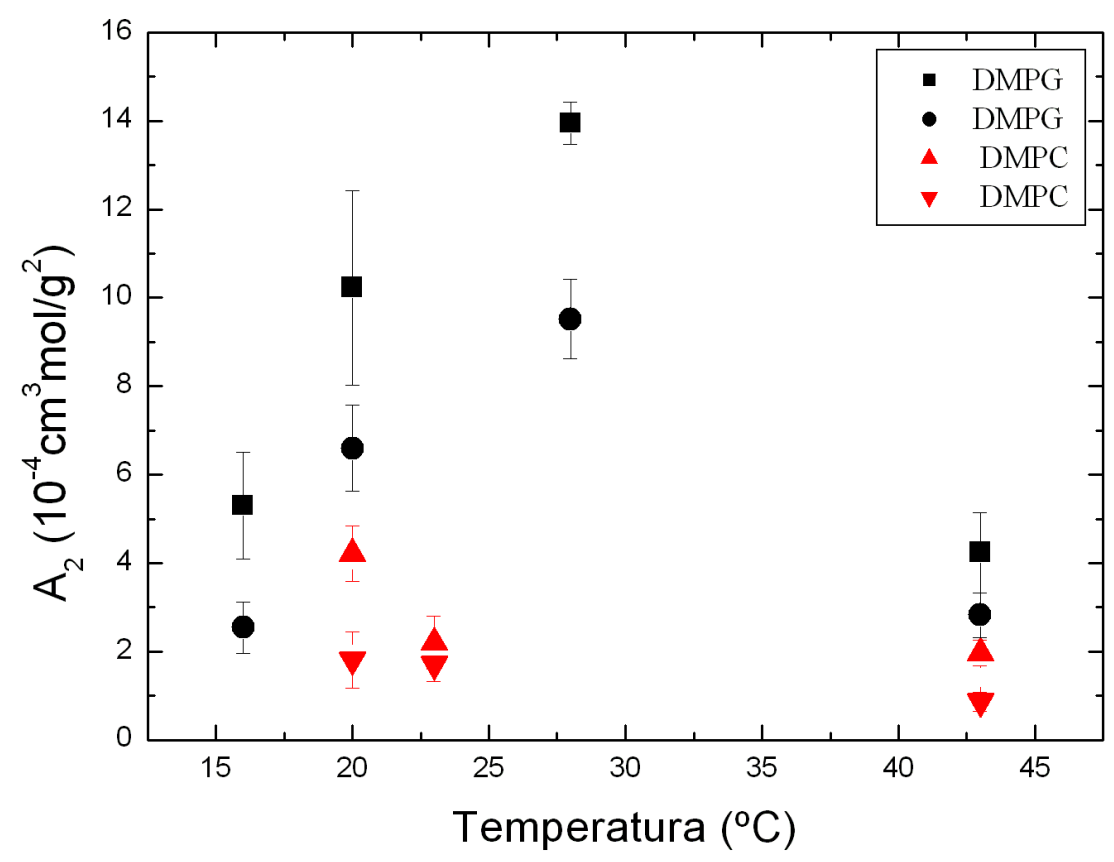

Figura 6.8: Comparação do segundo coeficiente do virial obtido para os lipídios DMPG e DMPC, pela análise de Zimm em diferentes temperaturas. 
O segundo coeficiente do virial, $A_{2}$, está relacionado à expansão da pressão osmótica, (conforme discutido no Capítulo 2). O aumento da pressão osmótica implica que as partículas interagentes tendem a se repelir, ( do contrário, sua diminuição implicaria na atração das partículas). Portanto, o $A_{2}$ está relacionado às interações entre os centros espalhadores. Tanto o DMPG quanto o DMPC apresentam valores de $A_{2}$ sempre positivos, o que indica interações repulsivas entre as vesículas.

Em geral, as fases gel e fluida do DMPG apresentam valores de $A_{2}$ maiores que as respectivas fases do DMPC. As maiores interações do DMPG podem estar relacionadas à repulsão eletrostática imposta pelas cabeças polares negativamente carregadas. No entanto, na região de transição do DMPG, $A_{2}$ é aproximadamente três vezes maior que nas fases gel e fluida. Esse aumento está de acordo com o aumento do grau de dissociação das cabeças polares encontrado nessa região, (Barroso et al., 2010), levando consequentemente ao aumento das interações repulsivas. Ainda de acordo com os resultados de condutividade elétrica (Barroso et al., 2010), o valor de $A_{2}$ aumenta de $T=20^{\circ} \mathrm{C}$ para $T=28^{\circ} \mathrm{C}$.

Os resultados do raio de giração dos gráficos de Zimm podem ser resumidos no gráfico da figura 6.9. O raio de giração pode ser relacionado ao tamanho e à forma da partícula.

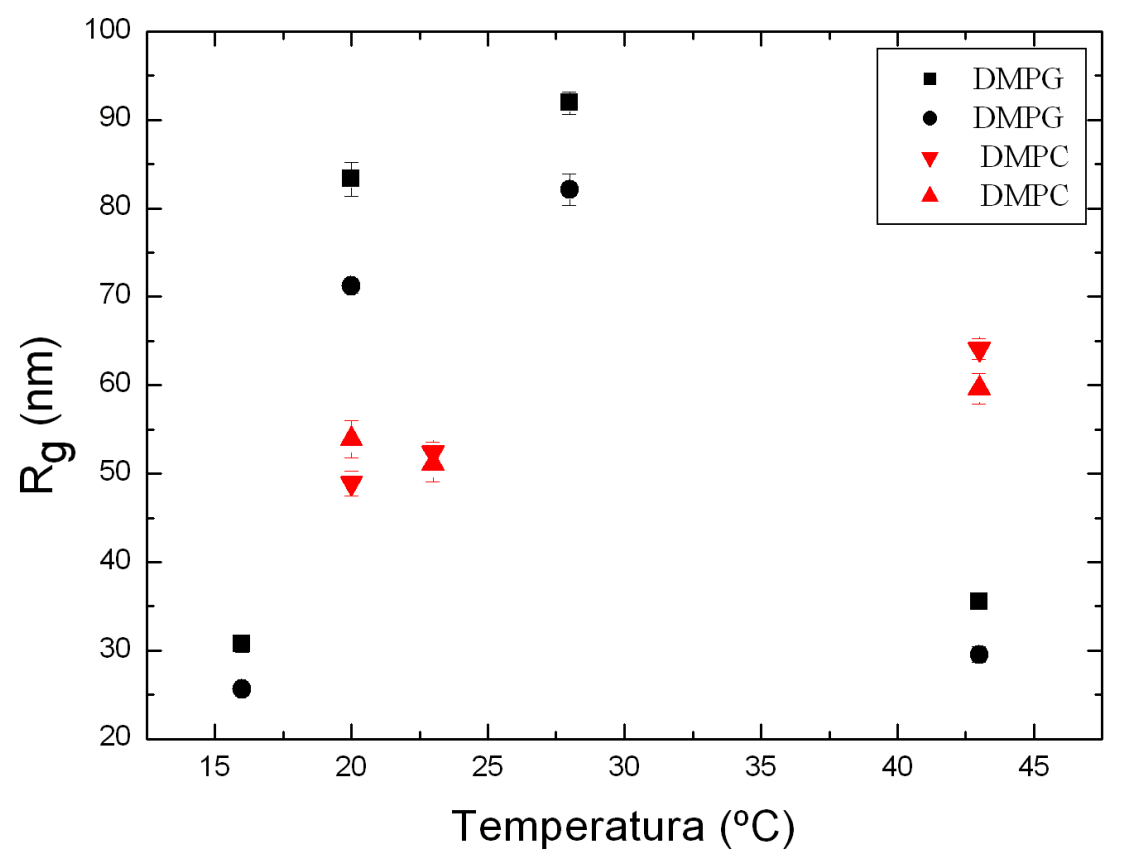

Figura 6.9: Comparação do raio de giração obtido para os lipídios DMPG e DMPC, pela análise de Zimm em diferentes temperaturas.

Admitindo que os agregados lipídicos são vesículas de geometria esférica, podemos modelar 
sua forma por meio de uma casca esférica, como demonstrado no Capítulo 2. Na equação (2.91) mostramos que o raio de giração para uma casca esférica (vesículas) é dado pela equação a baixo,

$$
R_{g}^{2}=\frac{3}{5} R_{e f}^{2} \frac{1-\left(1-r / R_{e f}\right)^{5}}{1-\left(1-r / R_{e f}\right)^{3}},
$$

onde $r$ corresponde à espessura da bicamada lipídica, e $R_{e f}$ o raio externo. Não é possível determinar analiticamente o $R_{e f}$ a partir desse polinômio, portanto a equação anterior foi resolvida numericamente. O método utilizado para calcular o $R_{e f}$ é simples: a função descrita pelo lado direito da igualdade é calculada numericamente para vários valores de $R_{e f}$ (com incremento de 0,01 , no intervalo $\left[R_{g} / 4,4 R_{g}\right]$ ). O valor de $R_{e f}$ que satisfaz a igualdade é solução da equação.

Para determinarmos os raios efetivos a partir dos raios de giração, nas diferentes temperaturas medidas, utilizamos diferentes valores para a espessura da bicamada $r$, de acordo com os valores encontrados na literatura. Para a fase gel do DMPC, $r=4,24 \mathrm{~nm}$, e para a fase fluida, $r=3,55 \mathrm{~nm}$, (Nagle e Tristram-Nagle, 2000; Marsh e Phil, 1990). Na temperatura de transição de fase do DMPC, utilizamos um valor intermediário dado pela média dos valores apresentados a cima. Admitimos que na transição de fase parte da bicamada tenha transicionado para a fase fluida e parte ainda se mantenha na fase gel, levando a uma média da espessura da bicamada representada pelo valor, $r=3,89 \mathrm{~nm}$. Para o DMPG utilizamos os mesmos valores discutidos anteriormente do DMPC. Na região de transição do DMPG foi utilizado o mesmo valor médio estimado para a temperatura de transição de fase do DMPC.

Os resultados do cálculo numérico são resumidos na tabela 6.5, para o DMPG e na tabela 6.6 para o DMPC.

Tabela 6.5: Raio efetivo do DMPG calculado numericamente para vesículas a partir do $R_{g}$ obtido pela análise de Zimm em diferentes temperaturas.

\begin{tabular}{|ccc|}
\hline Temperatura ${ }^{\circ} \mathrm{C}$ & $R_{\text {ef }}(\mathrm{nm})$ (grupo 1) & $R_{\text {ef }}(\mathrm{nm})$ (grupo 2) \\
\hline \hline 16 & $27,7 \pm 0,7$ & $32,7 \pm 0,9$ \\
20 & $73,1 \pm 0,8$ & $85 \pm 2$ \\
28 & $84 \pm 2$ & $94 \pm 1$ \\
43 & $31 \pm 1$ & $37,2 \pm 0,6$ \\
\hline \hline
\end{tabular}

Tanto para o DMPG quanto para o DMPC observamos um aumento do raio efetivo obtido através do raio de giração, da fase gel para a fase fluida. Esse resultado é semelhante ao aumento obtido com as análises de DLS, e será discutido adiante. No entanto, na região de transição do DMPG, o $R_{e f}$ é aproximadamente três vezes maior do que na fase gel, 
Tabela 6.6: Raio efetivo do DMPC calculado numericamente para vesículas a partir do $R_{g}$ obtido pela análise de Zimm em diferentes temperaturas.

\begin{tabular}{|ccc|}
\hline Temperatura ${ }^{\circ} \mathrm{C}$ & $R_{\text {ef }}(\mathrm{nm})$ (grupo 1) & $R_{\text {ef }}(\mathrm{nm})$ (grupo 2) \\
\hline \hline 20 & $54 \pm 1$ & $56 \pm 2$ \\
23 & $54 \pm 1$ & $54 \pm 2$ \\
43 & $65 \pm 1$ & $60 \pm 2$ \\
\hline \hline
\end{tabular}

por exemplo. Esses dados também serão discutidos mais à frente, juntamente com os resultados de DLS.

Em sistemas lipídicos, a transição de fase pode ser caracterizada por uma queda na turbidez, ou, em nossas análises, queda na intensidade da luz espalhada, como podemos observar nos gráficos da figura 6.10, para duas amostras diferentes.

A queda da intensidade da luz espalhada, da fase gel para a fase fluida é explicada por mudanças das propriedades ópticas da bicamada, (Disalvo, 1991; Yi e Mc Donald, 1973). Na fase fluida, a bicamada encontra-se mais hidratada, por isso a diferença de seu índice de refração com relação ao solvente é menor, como podemos observar na figura 6.10 para o DMPC.

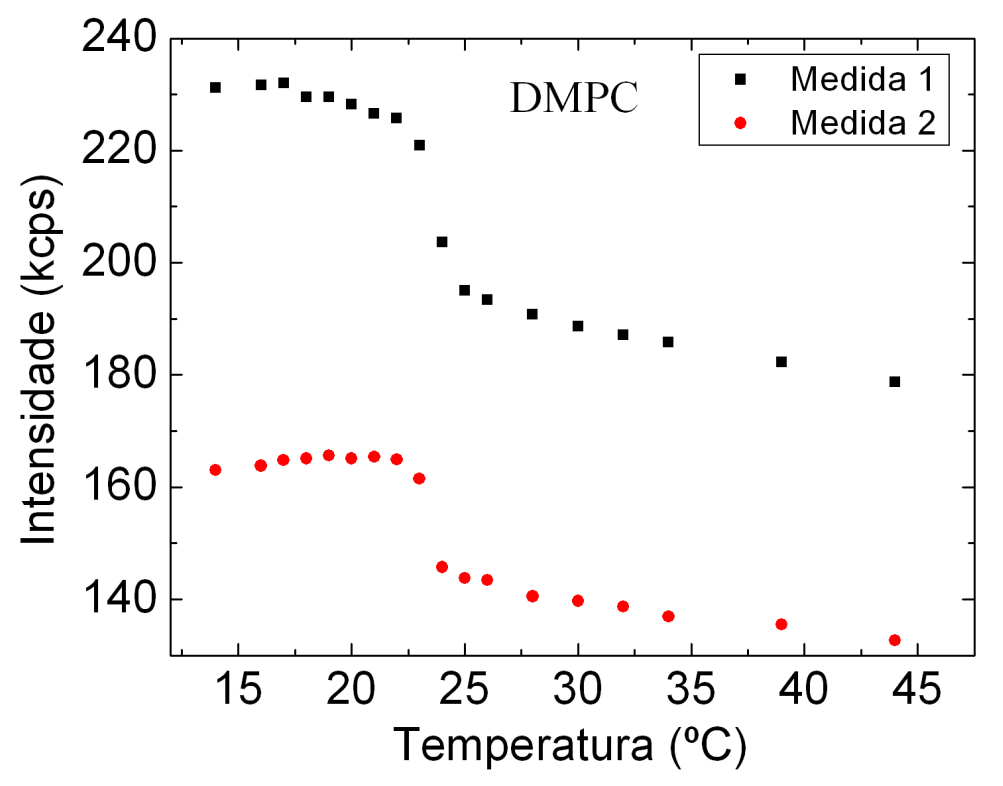

Figura 6.10: Intensidade da luz espalhada em função da temperatura, para a dispersão lipídica DMPC.

A mesma queda na intensidade da luz espalhada da fase gel para a fase fluída também é observada para o DMPG. No entanto, na região de transição do DMPG, podemos notar uma queda acentuada da intensidade da luz espalhada, figura 6.11, já observada na literatura. Além disso, vimos na figura 6.9 há um aumento considerável do raio de giração 
na mesma região, indicando um possível aumento ou mudança na forma do agregado lipídico.

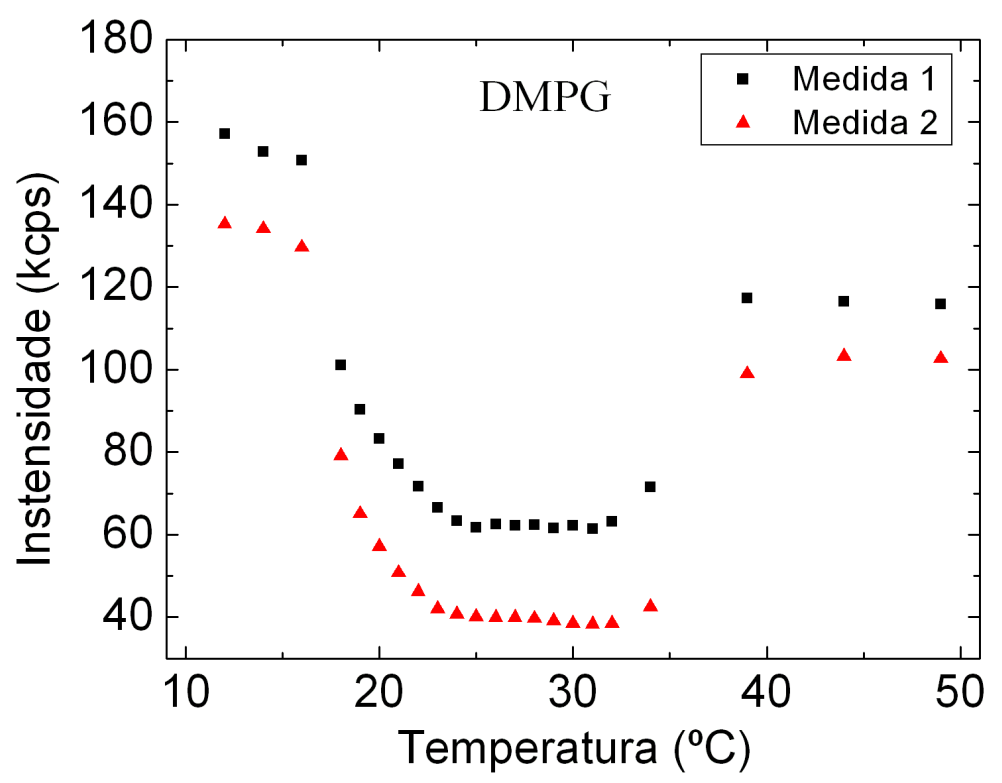

Figura 6.11: Intensidade da luz espalhada em função da temperatura, para as dispersão lipídica DMPG.

Um dos modelos propostos na literatura sugere que a dispersão lipídica de DMPG poderia ser formada por um conjunto de vesículas agregadas, que na região de transição estariam desagregadas. O alto grau dissociação das cabeças polares levaria ao aumento de cargas na superfície dos agregados, que por repulsão eletrostática desagregariam formando unidades menores. Neste modelo a queda na intensidade da luz espalhada foi atribuída à formação de pequenas vesículas. Nossos dados descartam este modelo, dado que encontramos um significativo aumento do $R_{g}$ na região de transição.

Sugerimos uma possível interpretação baseada na combinação dos dois resultados apresentados acima. A bicamada pode estar muito mais hidratada na região de transição do que na fase fluida, isto é, a diferença entre os índices de refração da bicamada e da água pode ser muito menor nesta região, do que nas fases gel e fluida. Portanto, podemos supor que a queda na intensidade da luz espalhada não precisa estar relacionada à formação de partículas menores, visto que o raio de giração apresenta um aumento bem significativo. Os resultados de DLS reforçam essa nossa hipótese, como veremos à frente. Quanto ao grande aumento do $R_{e f}$ calculado, voltaremos a essa discussão. 


\subsection{Espalhamento de Luz Dinâmico}

As medidas DLS, foram realizadas a vários ângulos e diversas concentrações. Utilizamos o métodos dos cumulantes de segunda ordem (conforme discutido no Capítulo 3 e no Anexo A), e primeiramente obtivemos o coeficiente de difusão translacional $D_{T}$, segundo a equação (3.12)

$$
D_{T}=\lim _{\Delta q \rightarrow 0} \frac{\Delta \Gamma}{\Delta q^{2}} \equiv \frac{d \Gamma}{d q^{2}}
$$

onde $\frac{d \Gamma}{d q^{2}}$ corresponde ao coeficiente angular da reta $\Gamma=D_{T} q^{2}$, conforme vimos no Capítulo 3.

Como também discutimos no Capítulo 3, a difusão de um centro espalhador pode ser influenciada pelas interações das partículas entre si. Portanto retomando a equação (3.14), podemos obter o coeficiente de difusão no regime infinitamente diluído, $D_{0}$

$$
D_{T}=D_{0}\left(1+k_{D} C\right)
$$

onde $k_{D}$ é o coeficiente do segundo termo da expansão (ordens superiores a $C$ foram desprezadas visto que já estamos trabalhando em um regime diluído).

Por fim aplicamos a relação de Stokes-Einstein para obter o diâmetro efetivo, $d_{e f}$, dos centros espalhadores, conforme equação (3.15)

$$
D_{0}=\frac{k_{B} T}{3 \pi \eta d_{e f}} .
$$

Deste modo, podemos traçar os gráficos $\Gamma \times q^{2}$, e a partir do coeficiente angular das retas, extrair os valores do coeficiente de difusão translacional $D_{T}$. Em seguida os valores de $D_{T}$ obtidos foram relacionados com as respectivas concentrações, e no limite $C \rightarrow 0$ (coeficiente linear), extraímos os valores de $D_{0}$ para as diferentes temperaturas medidas. Esse método foi realizado tanto para o DMPG quanto para o DMPC.

As próximas análises foram feitas para cinco diferentes concentrações. Estimamos, para o DMPG, a concentração de cada amostra pela média das medidas de dosagem de fosfato realizada para a análise de Zimm, tabela 5.1.

Os gráficos dados pela figura 6.12 referem-se às concentrações $(C=0,34 ; 0,86 ; 1,37 ; 1,67$; e 2,40 $m M$ ), para diferentes temperaturas. As medidas foram obtidas em diferentes ângulos de espalhamento $\theta=50^{\circ}, 70^{\circ}, 90^{\circ}, 110^{\circ}$ e $130^{\circ}$.

As medidas representadas na figura anterior, 6.12, foram repetidas para um segundo grupo de amostras, admitindo as mesmas concentrações médias. Para melhor controle 

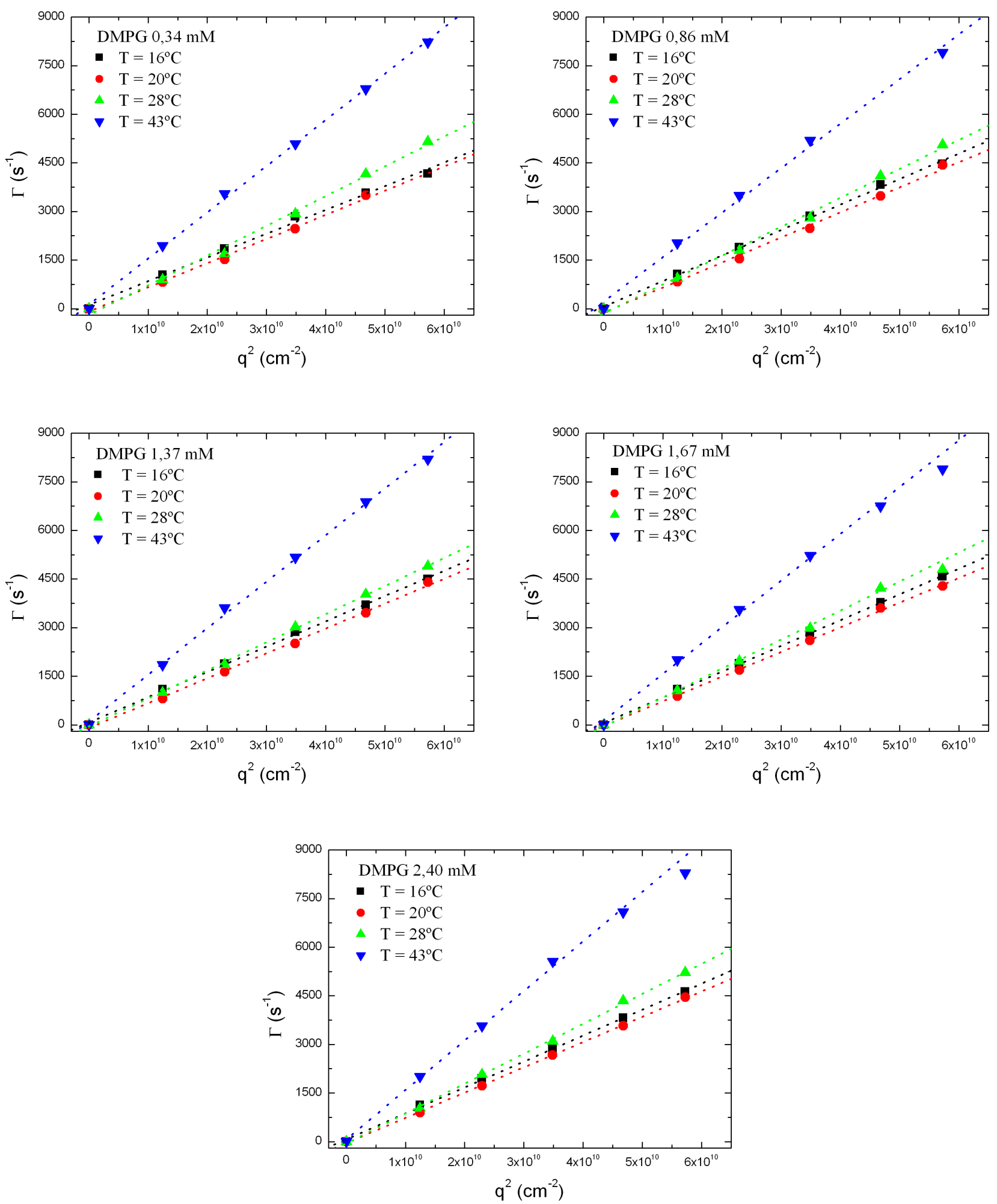

Figura 6.12: Cálculo do coeficiente de difusão translacional a partir do coeficiente angular das retas. Cada gráfico representa uma concentração de DMPG em diferentes temperaturas. Esse grupo de amostras foi denominado (grupo 1). 
das análises, as medidas foram coletadas em outros ângulos, $\theta=45^{\circ}, 60^{\circ}, 90^{\circ}, 120^{\circ} \mathrm{e}$ $135^{\circ}$. Podemos observar os resultados na figura 6.13.
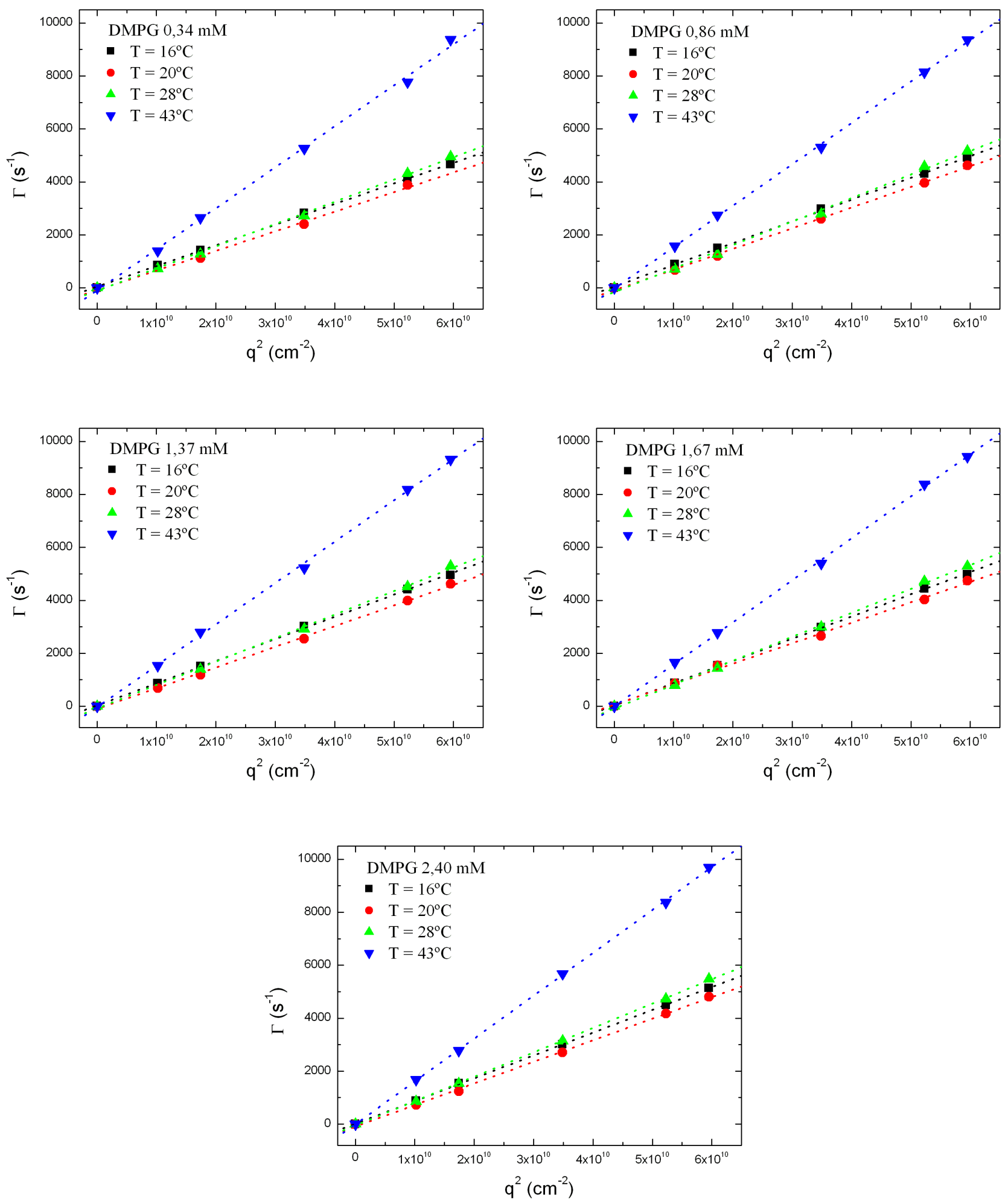

Figura 6.13: Cálculo do coeficiente de difusão translacional a partir do coeficiente angular das retas. Cada gráfico representa uma concentração de DMPG em diferentes temperaturas. Esse grupo de amostras foi denominado (grupo 2). 
Os gráficos da figura 6.14 ilustram um exemplo de como resultados obtidos com os dois grupos de amostras, figuras 6.12 (grupo 1) e 6.13 (grupo 2) concordam entre si. O exemplo mostra apenas uma concentração de DMPG, $C=1,37 \mathrm{mM}$, para diferentes temperaturas, $T=16$ e $43^{\circ} \mathrm{C}$ figura 6.14 (esquerda) e $T=20$ e $28^{\circ} \mathrm{C}$ (direita). Todas as demais concentrações apresentadas anteriormente nas figuras 6.12 (grupo 1) e 6.13 (grupo 2), também apresentam excelente concordância entre si.
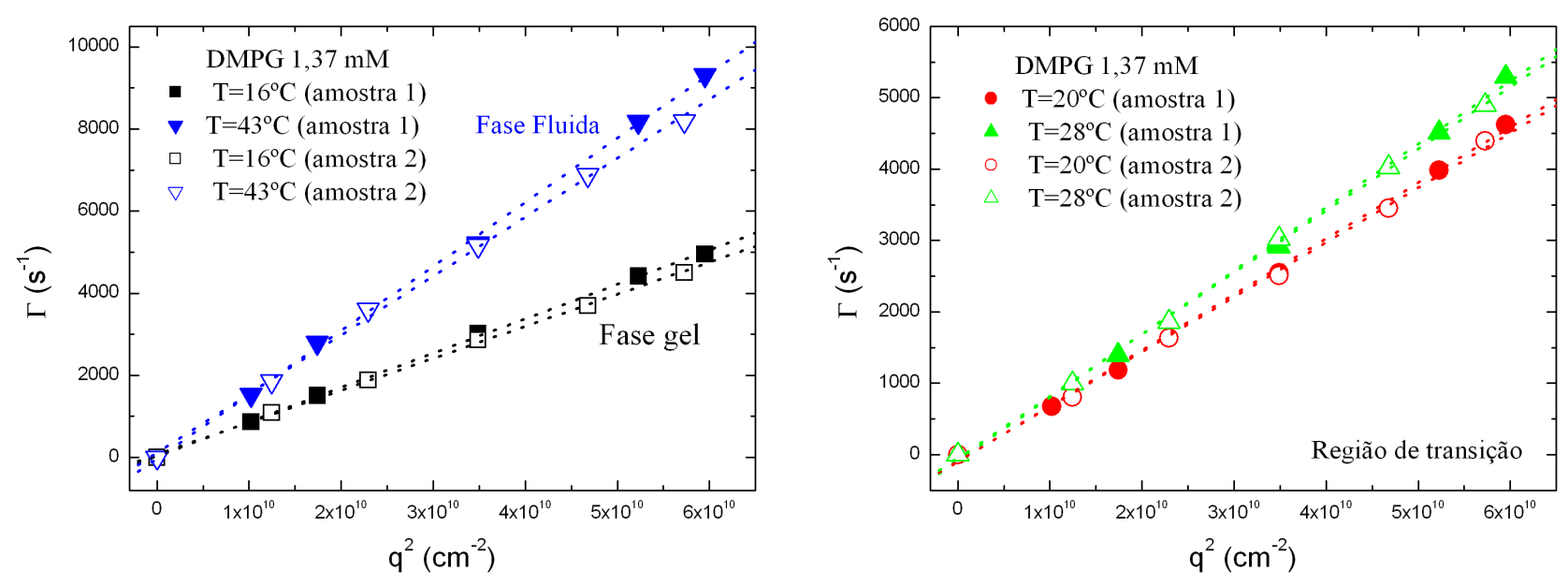

Figura 6.14: Exemplo da reprodutibilidade da medida de DLS para o DMPG.

Outro método foi utilizado para calcular os coeficientes de difusão translacional $D_{T}$. Esse outro método, obtem uma distribuição de taxas de decaimento, através da transformada de Laplace inversa, e este foi realizado no programa Gendist que obteve resultados muito similares aos apresentados aqui, e algumas de suas análises podem ser encontradas no apêndice B.

Com os valores dos coeficientes angulares das retas apresentadas nas figuras 6.12 (grupo 1) e 6.13 (grupo 2), podemos obter os coeficientes de difusão no regime de diluição infinita, $D_{0}$. Conforme realizado nos gráficos seguintes.

Das análises para o DMPG da figura 6.12 podemos estabelecer os limites dos gráficos da figura 6.15. Os erros em cada ponto foram estabelecidos por meio dos ajustes anteriores. 

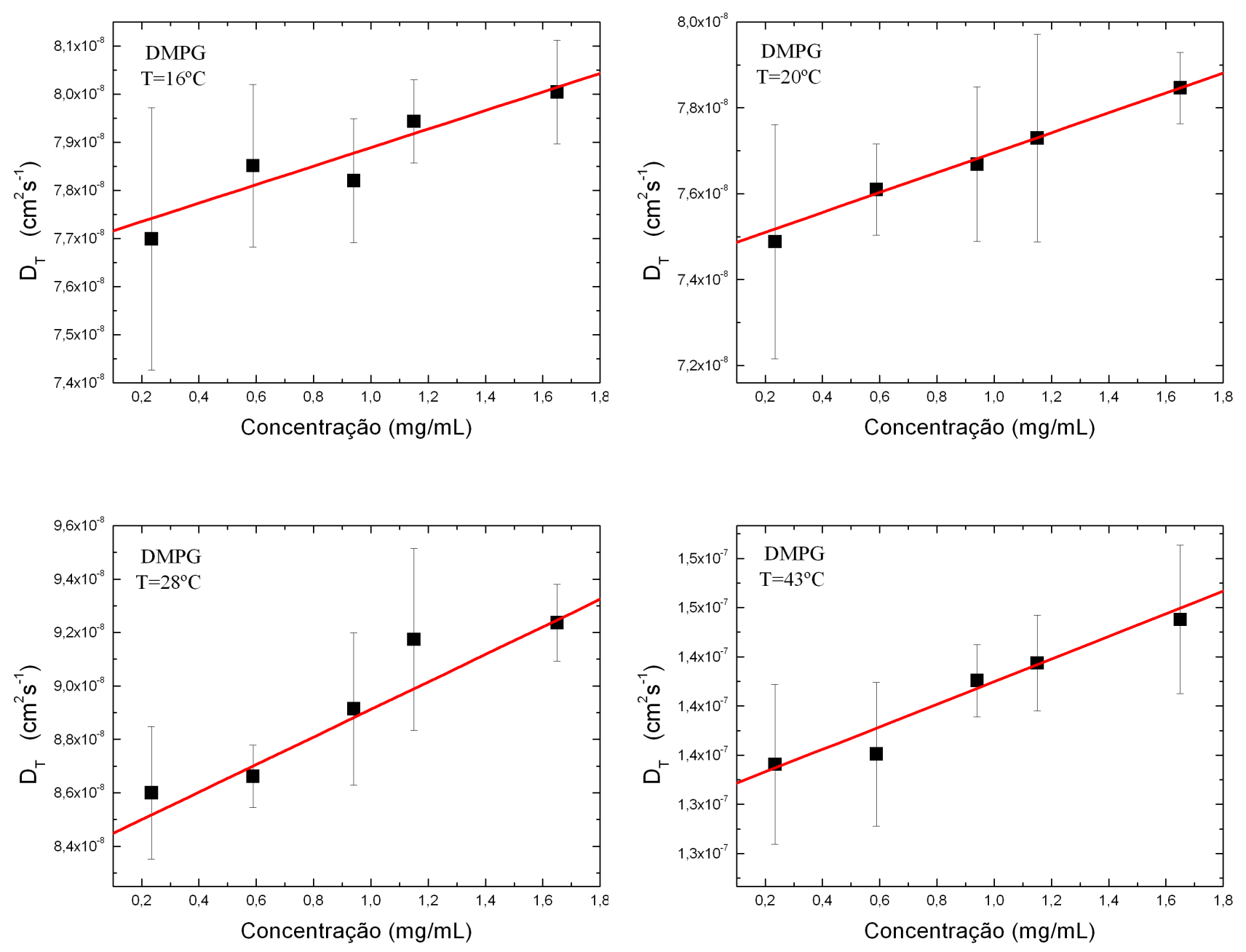

Figura 6.15: Determinação do coeficiente de difusão no limite de diluição infinita a partir do coeficiente linear das retas. Cada gráfico relaciona os $D_{T}$ das amostras do (grupo 1) de DMPG com as devidas contrações. Essa análise é realizada para as diferentes temperaturas medidas.

Os resultados de $D_{0}$ obtidos, e os respectivos diâmetros efetivos calculados através da relação de Stokes-Einstein (3.15), foram resumidos na tabela 6.7

Tabela 6.7: Coeficiente de difusão e diâmetro efetivo do DMPG no regime de diluição infinita - grupo 1 .

\begin{tabular}{|ccc|}
\hline Temperatura ${ }^{\circ} \mathrm{C}$ & $D_{0}\left(10^{-8} \mathrm{~cm}^{2} / \mathrm{s}\right)$ & $d_{\text {ef }}(\mathrm{nm})$ \\
\hline \hline 16 & $7,7 \pm 0,1$ & $50 \pm 1$ \\
20 & $7,5 \pm 0,1$ & $57 \pm 1$ \\
28 & $8,4 \pm 0,1$ & $63 \pm 1$ \\
43 & $13,6 \pm 0,2$ & $53 \pm 2$ \\
\hline \hline
\end{tabular}


Para o segundo grupo de amostra de DMPG temos os limites $C \rightarrow 0$ dados pelos gráficos da figura 6.16, e os resultados resumidos na tabela 6.8.
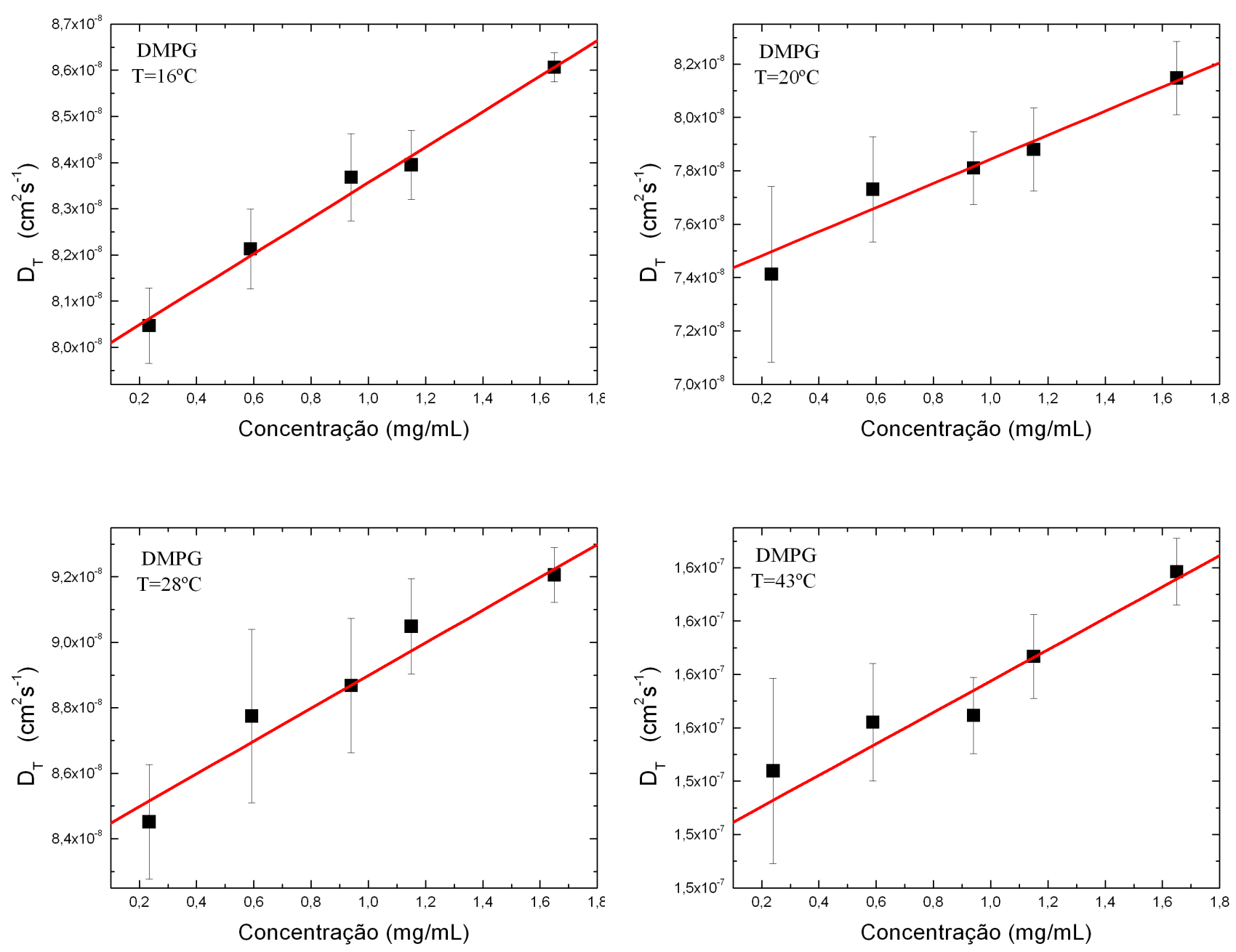

Figura 6.16: Determinação do coeficiente de difusão no limite de diluição infinita a partir do coeficiente linear das retas. Cada gráfico relaciona os $D_{T}$ das amostras do (grupo 2) de DMPG com as devidas contrações. Essa análise é realizada para as diferentes temperaturas medidas.

Tabela 6.8: Coeficiente de difusão e diâmetro efetivo do DMPG no regime de diluição infinita - grupo 2.

\begin{tabular}{|ccc|}
\hline Temperatura ${ }^{\circ} \mathrm{C}$ & $D_{0}\left(10^{-8} \mathrm{~cm}^{2} / \mathrm{s}\right)$ & $d_{\text {ef }}(\mathrm{nm})$ \\
\hline \hline 16 & $7,97 \pm 0,07$ & $47,9 \pm 0,4$ \\
20 & $7,3 \pm 0,2$ & $58 \pm 1$ \\
28 & $8,4 \pm 0,1$ & $63 \pm 1$ \\
43 & $15,2 \pm 0,2$ & $51,4 \pm 0,7$ \\
\hline \hline
\end{tabular}

O método dos Cumulantes segunda ordem fornece também o índice de polidisepersão do sistema, que está relacionada à variância da distribuição de $\Gamma$ (conforme mostrado no apêndice A). Na tabela 6.9 relacionamos os valores médios dos índices de polidisepersão 
do DMPG nas diferentes temperaturas.

Tabela 6.9: Média do índice de polidispersão do DMPG obtida pelo método dos Cumulantes de segunda ordem.

\begin{tabular}{|cc|}
\hline Temperatura ${ }^{\circ} C$ & índice de polidispersão \\
\hline \hline 16 & $0,07 \pm 0,06$ \\
20 & $0,14 \pm 0,05$ \\
28 & $0,17 \pm 0,04$ \\
43 & $0,06 \pm 0,05$ \\
\hline \hline
\end{tabular}

Podemos observar que a região de transição é um pouco mais polidispersa do que as fases gel e fluida.

O mesmo tratamento de dados foi utilizado para o DMPC. As concentrações das próximas análises são dadas pela média das medidas de dosagem de fosfato realizadas para o DMPC na análise de Zimm, tabela 5.2.

Os gráficos da figura 6.17 mostram as análises do coeficiente de difusão translacional, para as diferentes concentrações de $\operatorname{DMPC}(C=0,49 ; 0,88 ; 1,36 ; 1,85$ e $2,27 m M)$. As medidas foram realizadas nas diferentes fases do DMPC (gel e fluida), e na temperatura de transição de fase. A correlação da luz espalhada foi coletada a diferentes ângulos de espalhamento, $\theta=50^{\circ}, 70^{\circ}, 90^{\circ}$ e $110^{\circ}$.
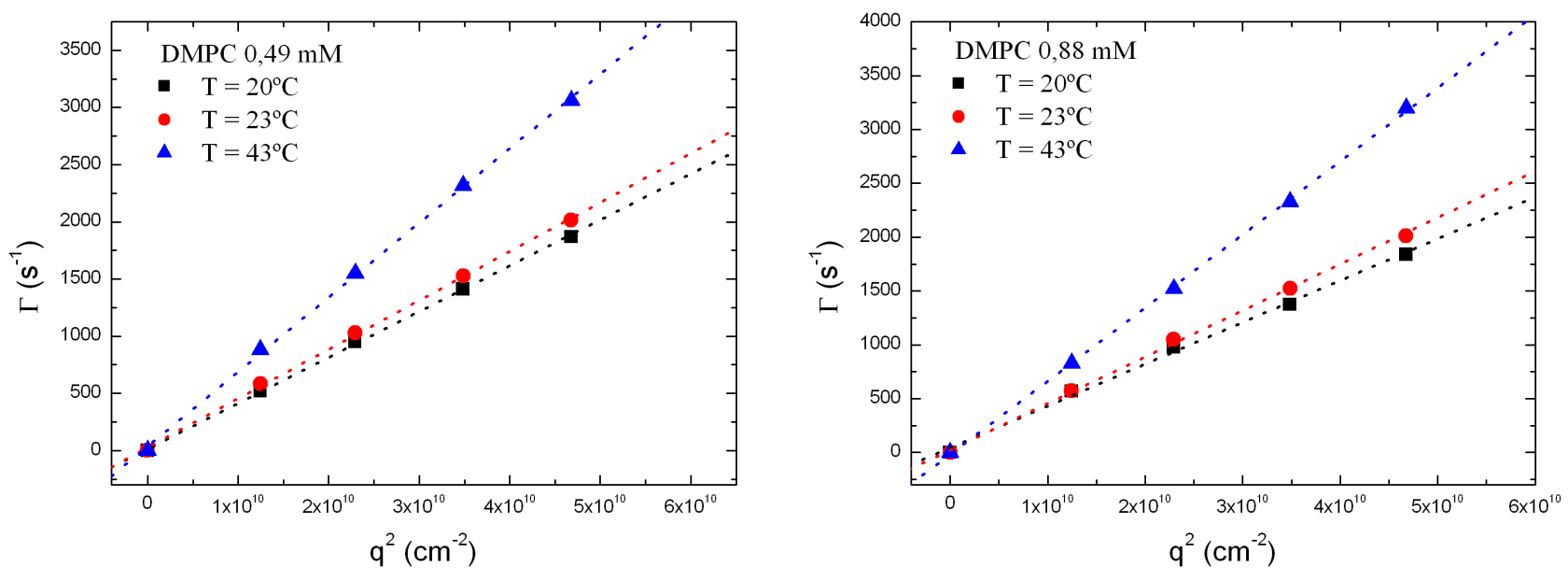

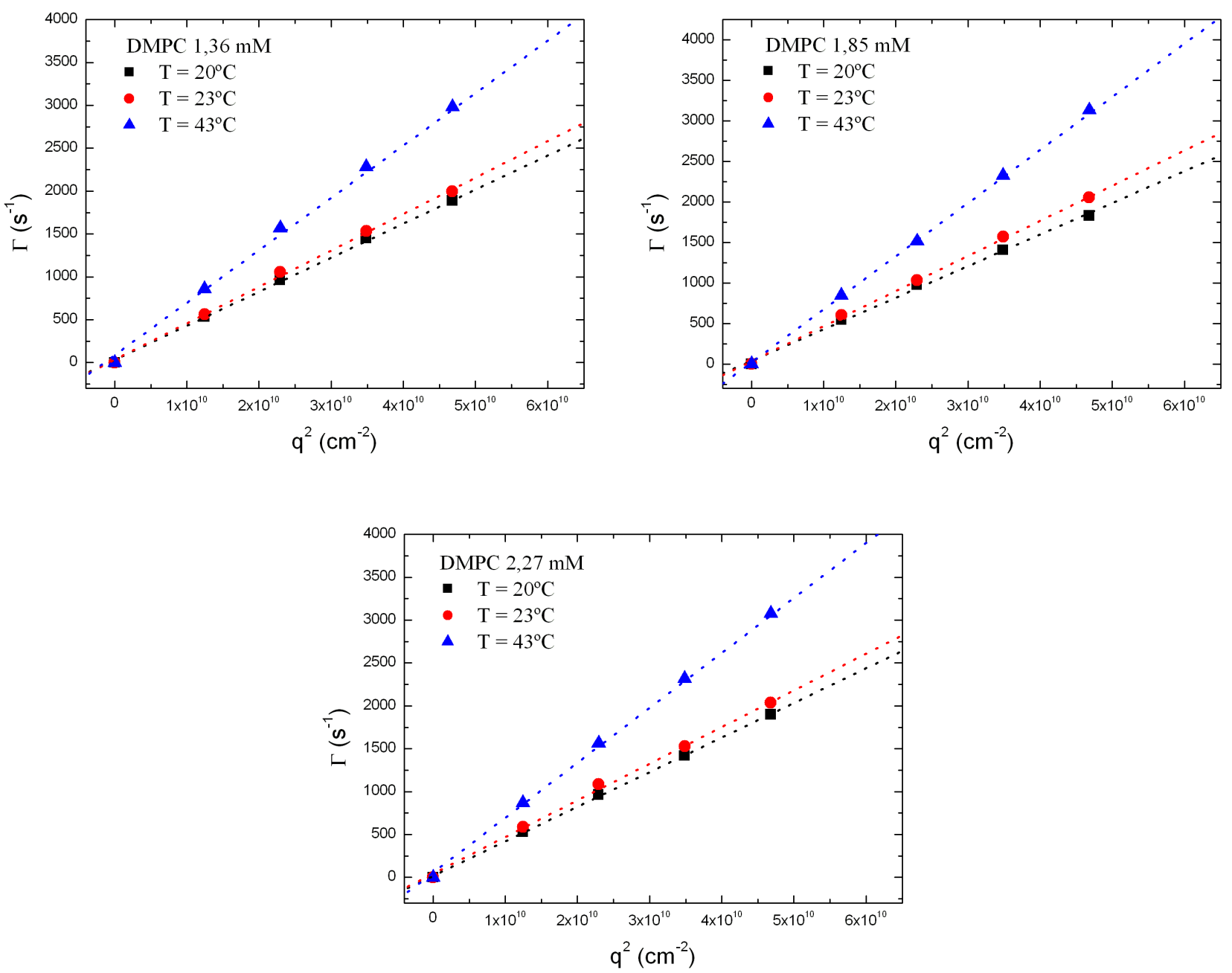

Figura 6.17: Cálculo do coeficiente de difusão translacional a partir do coeficiente angular das retas. Cada gráfico representa uma concentração de DMPC em diferentes temperaturas. Esse grupo de amostras foi denominado (grupo 1).

As medidas de DLS para o DMPC foram repetidas para diferentes ângulos de espalhamento, $\theta=45^{\circ}, 60^{\circ}, 90^{\circ}, 120^{\circ}$ e $135^{\circ}$, e os resultados podem ser observados na figura 6.18 . 

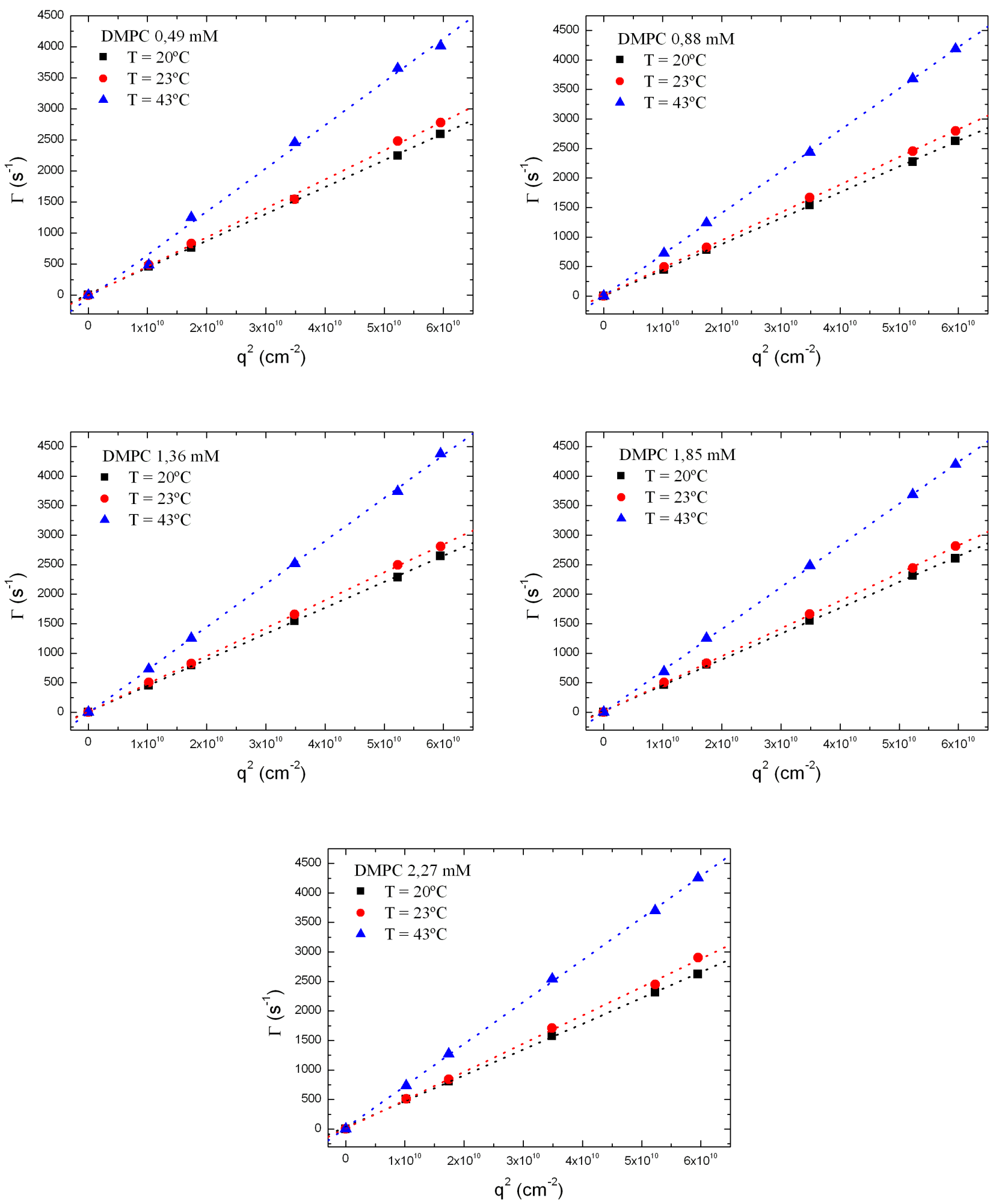

Figura 6.18: Cálculo do coeficiente de difusão translacional a partir do coeficiente angular das retas. Cada gráfico representa uma concentração de DMPC em diferentes temperaturas. Esse grupo de amostras foi denominado (grupo 2). 
Os dois grupos de amostras representados pelas figuras 6.17 (grupo 1) e 6.18 (grupo 2), apresentam resultados semelhantes. Essa concordância dos resultados é ilustrada no exemplo dado pela figura 6.19, para uma dada concentração de DMPC, no caso $C=$ $1,36 m M$.
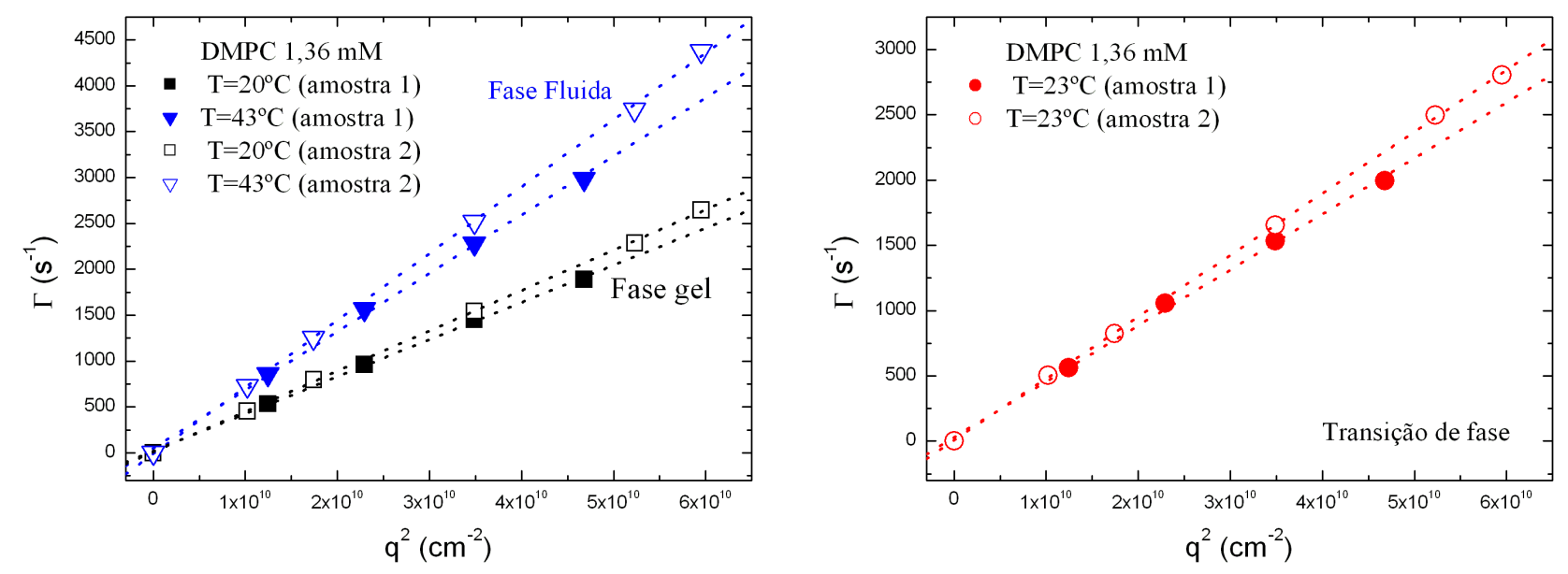

Figura 6.19: Exemplo da reprodutibilidade da medida de DLS para o DMPC.

Para todas as medidas dos dois grupos de amostras, com a análise dos Cumulantes, figuras 6.17 e 6.18, obtivemos boa concordância dos resultados, porém as amostras de DMPC são mais diferentes entre si, que as amostras de DMPG (em concentrações semelhantes). Essa diferença pode ser devida ao processo de extrusão do DMPC, que é mais trabalhoso e exige mais esforço. A comparação dos resultados pelas diferentes análises, Cumulantes e método utilizado com o programa Gendist, também apresenta boa concordância e pode ser observada no apêndice B.

Os valores $D_{T}$ para o DMPC também são relacionados às respectivas concentrações, e nos gráficos 6.20 e 6.21 extraímos os valores de $D_{0}$, para a fase gel e fluida, e temperatura de transição.

As próximas análises da figura 6.20 referem-se às medidas apresentadas na figura 6.17. 

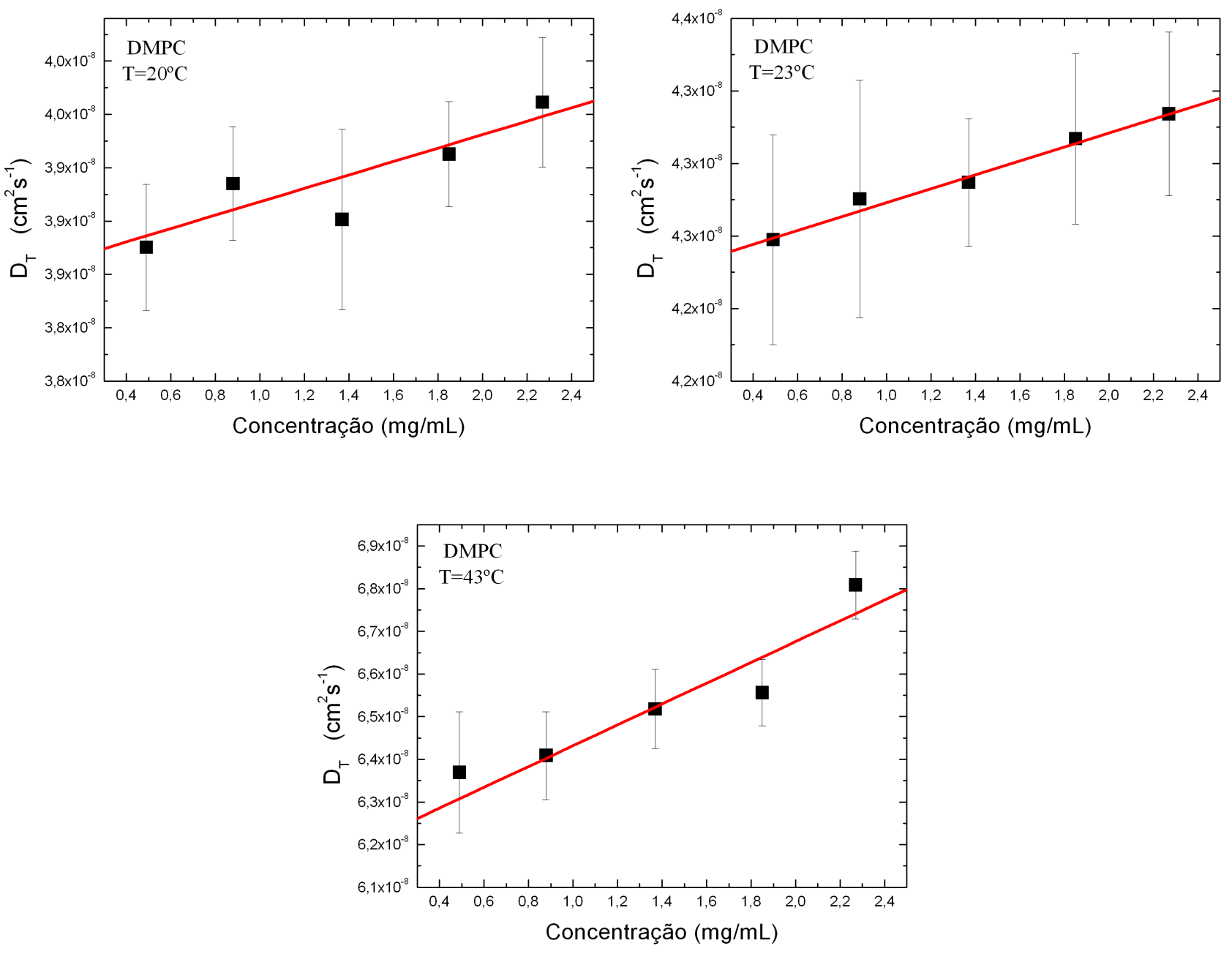

Figura 6.20: Determinação do coeficiente de difusão no limite de diluição infinita a partir do coeficiente linear das retas. Cada gráfico relaciona os $D_{T}$ das amostras do (grupo 1) de DMPC com as devidas contrações. Essa análise é realizada para as diferentes temperaturas medidas.

Tabela 6.10: Coeficiente de difusão e diâmetro efetivo do DMPC no limite de diluição infinita - grupo 1.

\begin{tabular}{|ccc|}
\hline Temperatura ${ }^{\circ} \mathrm{C}$ & $D_{0}\left(10^{-8} \mathrm{~cm}^{2} / \mathrm{s}\right)$ & $d_{\text {ef }}(\mathrm{nm})$ \\
\hline \hline 20 & $3,86 \pm 0,06$ & $111 \pm 2$ \\
23 & $4,22 \pm 0,07$ & $110 \pm 2$ \\
43 & $6,2 \pm 0,1$ & $126 \pm 3$ \\
\hline \hline
\end{tabular}


Para as medidas dadas pelo gráfico 6.18, temos as análises dos gráficos dado pela figura 6.21.
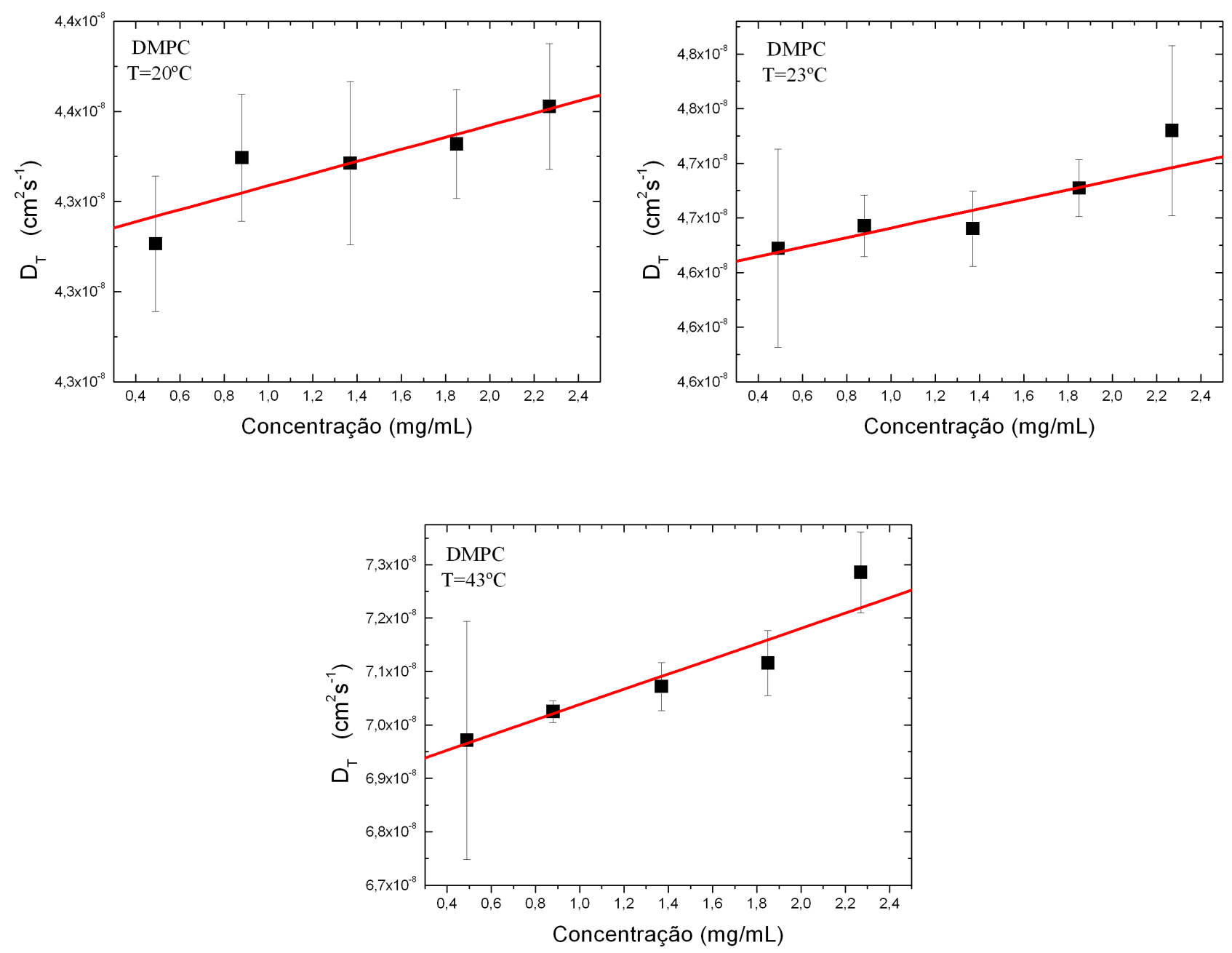

Figura 6.21: Determinação do coeficiente de difusão no limite de diluição infinita a partir do coeficiente linear das retas. Cada gráfico relaciona os $D_{T}$ das amostras do (grupo 2) de DMPC com as devidas contrações. Essa análise é realizada para as diferentes temperaturas medidas.

Tabela 6.11: Coeficiente de difusão e diâmetro efetivo do DMPC no regime de diluição infinita - grupo 2.

\begin{tabular}{|ccc|}
\hline Temperatura ${ }^{\circ} \mathrm{C}$ & $D_{0}\left(10^{-8} \mathrm{~cm}^{2} / \mathrm{s}\right)$ & $d_{\text {ef }}(\mathrm{nm})$ \\
\hline \hline 20 & $4,33 \pm 0,04$ & $99 \pm 1$ \\
23 & $4,65 \pm 0,05$ & $100 \pm 1$ \\
43 & $6,90 \pm 0,05$ & $113 \pm 1$ \\
\hline \hline
\end{tabular}


Os valores médios da índice de polidispersão para o DMPC nas diferentes temperaturas, são resumidos na tabela 6.12 .

Tabela 6.12: Média do índice de polidispersão do DMPG obtida pelo método dos Cumulantes de segunda ordem).

\begin{tabular}{|cc|}
\hline Temperatura ${ }^{\circ} C$ & índice de polidispersão \\
\hline \hline 20 & $0,09 \pm 0,02$ \\
23 & $0,07 \pm 0,02$ \\
43 & $0,10 \pm 0,03$ \\
\hline \hline
\end{tabular}

Interessante notar que os valores da índice de polidispersão do DMPC são da ordem dos valores do DMPG, na fase gel e fluida, e menores que a região de transição.

Podemos observar tanto para o DMPG quanto para o DMPC, que as concentrações mais diluídas estão bem próximas do regime de diluição infinita $C \rightarrow 0$. Nessas concentrações não há praticamente influência de interações entre partículas na difusão. A constante $k_{D}$ dada na expansão do coeficiente de difusão possui significado físico semelhante ao $A_{2}$, (Berne, 2000), na expansão da pressão osmótica, como vimos no Capítulo 2. Embora não iremos entrar em detalhes, a constante $k_{D}$ responde de forma análoga ao $A_{2}$ visto nas análises de Zimm. Podemos observar dos gráficos anteriores que $k_{D}$ assume sempre valores positivos, dado pelos coeficientes angulares das retas.

Das análises de DLS, podemos resumir os coeficientes de difusão $D_{0}$, no gráfico 6.22.
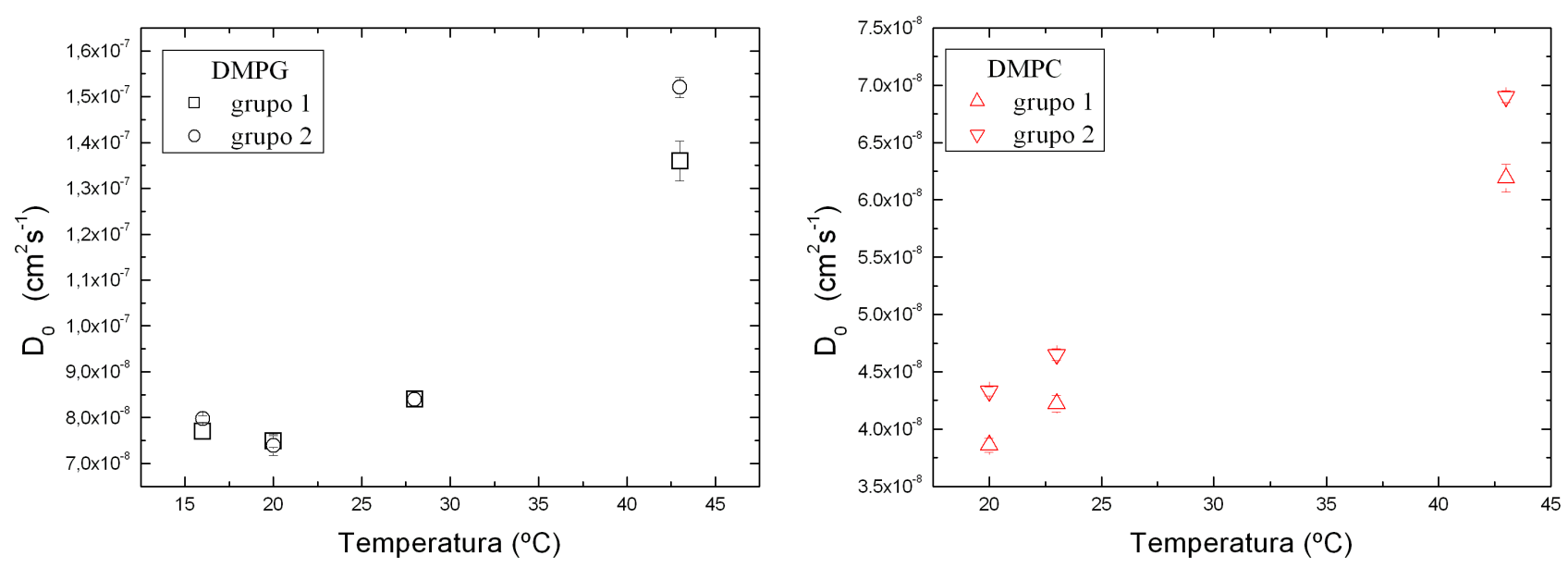

Figura 6.22: Coeficientes de difusão no regime de diluição infinita, em função da temperatura, para o DMPG (esquerda) e DMPC (direita).

Para uma partícula de raio constante, o aumento da temperatura leva a um aumento 
da difusão dessa partícula em um determinado meio, pois

$$
D_{T} \sim T / \eta
$$

onde a viscosidade do meio, $\eta$, decai com a temperatura, e portanto o coeficiente de difusão é proporcional a uma potência da temperatura, ou seja $D_{T} \sim T^{a}$, onde " $a^{\prime \prime}$ é necessariamente positivo.

Para o DMPC, o coeficiente de difusão aumenta com a temperatura, mesmo com o raio da partícula não sendo preservado constante, para o devido valor da viscosidade. O mesmo pode ser observado da fase gel do DMPG para sua fase fluida. Entretanto, o coeficiente de difusão na região de transição do DMPG apresenta um comportamento diferenciado, embora não tenhamos observado uma queda significativa em relação à fase gel (em valor absoluto), observamos que algum fator dificulta a difusão dos agregados lipídicos nesta fase.

Os diâmetros efetivos obtidos pela relação de Stokes-Einstein, podem ser observados em função da temperatura na figura 6.23.
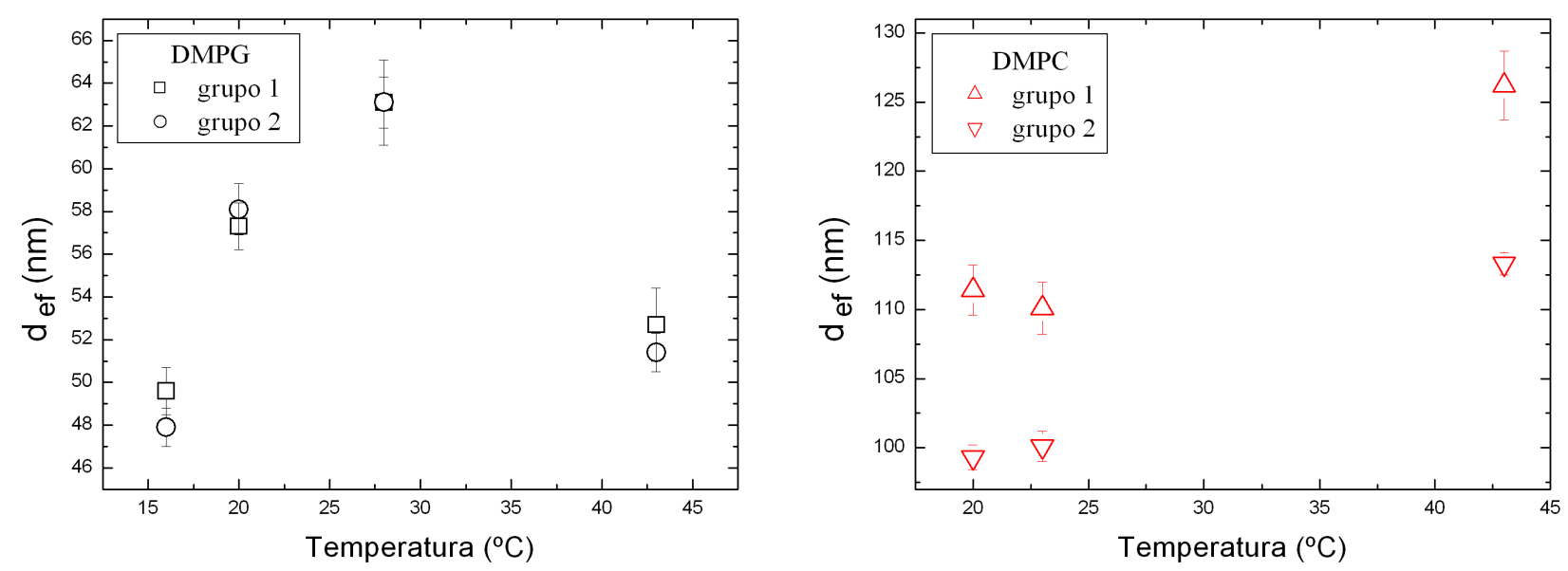

Figura 6.23: Diâmetro efetivo, obtido dos resultados DLS a partir da relação de Stokes-Einstein, no regime de diluição infinita, em função da temperatura, para o DMPG (esquerda) e DMPC (direita).

O aumento do diâmetro efetivo do DMPG e DMPC da fase fluida em relação à fase gel pode estar relacionado ao aumento da área ocupada por cabeça polar de lipídeo, $A_{C P L}$, como discutiremos adiante. Entretanto, o DMPG apresenta um aumento do diâmetro efetivo na região de transição, maior do que o aumento provocado na transição gel-fluido. Como vimos, na figura 6.22 esse aumento está relacionado aos valores relativamente baixos 
para os coeficientes de difusão nesta região de transição.

Observamos um aumento do diâmetro efetivo na região de transição do DMPG, no entanto, em menor escala quando comparado ao resultado obtido no SLS. Ambos os métodos, SLS e DLS, evidenciam um aumento do tamanho efetivo se considerarmos que o agregado seja preservado em uma geometria esférica, nesta região.

\subsection{Comparação dos resultados, SLS e DLS}

Podemos comparar os raios efetivos obtidos por SLS e DLS. Os raios efetivos do SLS foram calculados a partir do raio de giração obtido das análises de Zimm, esses dados podem ser observados nas tabelas 6.5 e 6.6 para o DMPG e DMPC respectivamente. Os raios efetivos do DLS, $R_{e f} \equiv d_{e f} / 2$, foram obtidos das medidas do coeficiente de difusão, dos centros espalhadores, e seus valores podem ser observados pelas tabelas 6.7, 6.8, 6.10 e 6.11 .
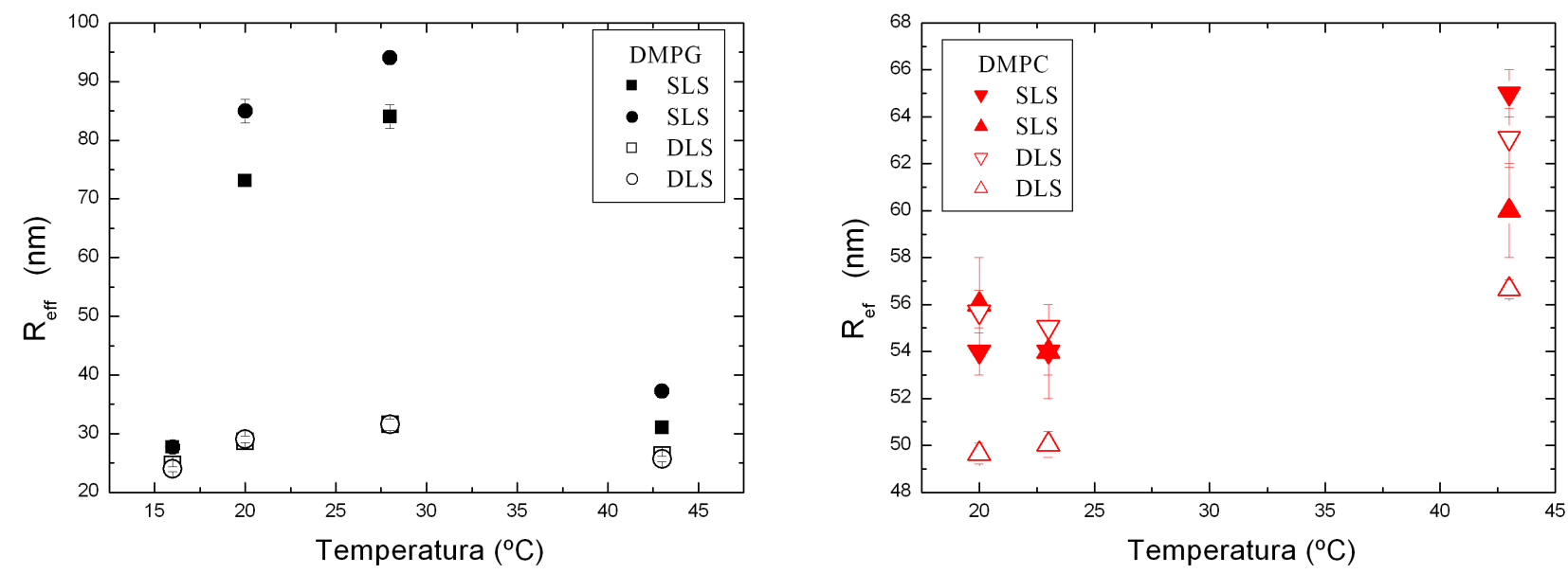

Figura 6.24: Comparação entre os raios efetivos obtidos por SLS e DLS para o DMPG e DMPC, em função da temperatura.

Os valores médios dos gráficos da figura 6.24 são resumidos nas tabelas 6.13, para o DMPG e 6.14, para o DMPC.

Os raios efetivos obtidos por SLS e DLS para o DMPC concordam dentro das flutuações de tamanhos de amostra para amostra, conforme ilustra a figura 6.24 à direita. Portanto, podemos observar que ambos os métodos, estático e dinâmico, são capazes de tratar o 
Tabela 6.13: Raio efetivo obtido a partir da média das medidas realizadas por DLS e SLS para o DMPG.

\begin{tabular}{|ccc|}
\hline Temperatura ${ }^{\circ} \mathrm{C}$ & $R_{\text {ef }}(\mathrm{nm})-\mathrm{SLS}$ & $R_{\text {ef }}(\mathrm{nm})-\mathrm{DLS}$ \\
\hline \hline 16 & $30 \pm 4$ & $25 \pm 1$ \\
20 & $79 \pm 8$ & $29 \pm 1$ \\
28 & $89 \pm 7$ & $32 \pm 1$ \\
43 & $34 \pm 4$ & $26 \pm 1$ \\
\hline
\end{tabular}

Tabela 6.14: Raio efetivo obtido a partir da média das medidas realizadas por DLS e SLS para o DMPC.

\begin{tabular}{|ccc|}
\hline Temperatura ${ }^{\circ} \mathrm{C}$ & $R_{\text {ef }}(\mathrm{nm})-\mathrm{SLS}$ & $R_{\text {ef }}(\mathrm{nm})-\mathrm{DLS}$ \\
\hline \hline 20 & $55 \pm 2$ & $52 \pm 4$ \\
23 & $54 \pm 2$ & $52 \pm 4$ \\
43 & $63 \pm 4$ & $60 \pm 5$ \\
\hline
\end{tabular}

sistema DMPC, e as diferentes formas de análises que convergem para o mesmo resultado.

Entretanto para o DMPG não temos a mesma concordância. Para as fases gel e fluida os valores dos $R_{e f}$ obtidos por SLS e DLS apresentam uma diferença de aproximadamente $30 \%$, em média.

Para compor as nossas discussões vamos trazer um resultado experimental obtido pelo grupo mas que não será discutido em detalhes neste trabalho. Medidas de permeabilidade à sacarose foram realizadas pelo aluno de doutorado Rafael Barroso (trabalho em andamento), e foi observado que o DMPG, nas fases gel e fluida, apresenta maior permeabilidade ao açúcar que o DMPC, nas respectivas fases. Isso indica que a bicamada de DMPG é mais "frouxa", e mais permeável ao solvente que a bicamada de DMPC ${ }^{2}$. Então sugerimos que a força de atrito viscoso imposta pelo solvente seja menor para o DMPG, devido a essa alta permeabilidade, levando a um coeficiente de difusão aparente maior, e consequentemente a tamanhos efetivos ligeiramente menores do que os obtidos por SLS, que não depende da difusão do agregado.

Dos resultados da figura 6.24 ainda podemos observar que os raios efetivos da fase fluida apresentam um aumento em relação à fase gel, tanto para o DMPC quanto para o DMPG. Esse aumento é inclusive observado por ambos os métodos analisados, SLS e DLS.

Podemos calcular a razão das áreas, do agregado lipídico, na fase fluida com relação à

\footnotetext{
${ }^{2}$ Esse experimento só pode ser realizado nas fases gel e fluida devido à alta viscosidade do DMPG na região de transição.
} 
fase gel. Visto que a área dos agregados nas respectivas fases é dada por

$$
A^{\text {fluida }}=4 \pi\left(R_{\text {ef }}^{\text {fluida }}\right)^{2} \quad, \quad A^{\text {gel }}=4 \pi\left(R_{e f}^{\text {gel }}\right)^{2},
$$

então a razão $A^{\text {fluida }} / A^{\text {gel }}$ nos fornece o aumento de área observado.

Na tabela 6.17 listamos as razões $A^{\text {fluida }} / A^{\text {gel }}$ para os valores das tabelas 6.13 e 6.14 , tanto para o DMPG quanto para o DMPC, separando o método analisado, SLS ou DLS.

Tabela 6.15: Razão entre a área ocupada por cabeça polar de lipídio na fase gel e fluida.

\begin{tabular}{|c||cc|}
\hline & $A_{D M P G}^{\text {fluida }} / A_{D M P G}^{\text {gel }}$ & $A_{D M P C}^{\text {fluida }} / A_{D M P C}^{\text {gel }}$ \\
\hline \hline SLS & 1,27 & 1,33 \\
DLS & 1,15 & 1,29 \\
\hline \hline
\end{tabular}

Os dados da tabela 6.17 nos permitem observar que a área do DMPC, por exemplo, teve um aumento de aproximadamente $30 \%$ da fase gel para a fase fluida. Para o DMPG o SLS observou um aumento semelhante, diferentemente do DLS.

Esse aumento de área observado para os agregados lipídicos da fase gel para a fase fluida pode ser relacionado ao aumento da área ocupada por cabeça polar de lipídio. Na fase gel um lipídio ocupa uma área de $0,48 \mathrm{~nm}^{2}$ enquanto que na fase fluida, o mesmo lipídio passa a ocupar uma área de 0,60 $\mathrm{nm}^{2}$ (Nagle e Tristram-Nagle, 2000; Marsh e Phil, 1990). A área ocupada por lipídio da fase fluida apresenta um aumento de aproximadamente 25\% com relação a fase gel.

Portanto, sugerimos que o aumento de tamanho observado da fase gel para a fase fluida, dos agregados lipídicos em estudo está relacionado ao aumento da área ocupada por lipídio, nas respectivas fases.

Entretanto, na região de transição do DMPG há uma discrepância dos resultados obtidos pelos diferentes métodos. Sugerimos duas interpretações possíveis. O agregado lipídico pode deformar na região de transição, e o cálculo do raio efetivo a partir de uma vesícula esférica passa a ser superestimado, considerando o valor encontrado para o raio de giração. Outra hipótese, a medida do diâmetro efetivo por DLS pode estar subestimada por algum outro motivo. Voltaremos a essa discussão adiante.

Observando as análises realizadas pelos diferentes métodos, SLS e DLS, para o DMPC e o DMPG, notamos que a combinação dessas medidas permite fazer avaliações mais completas do sistema. Pois, como vemos no caso do DMPG, a diferença dos resultados na região de transição nos conduz a maiores investigações e análises. 


\subsection{Medidas de anisotropia do centro espalhador}

Na região de transição do DMPG observamos que o raio de giração obtido pelos gráficos de Zimm apresentam valores muito maiores do que os obtidos para as fases gel e fluida. Além disso, observamos também que há uma grande diferença entre os valores dos raios efetivos calculados a partir do $R_{g}$ por SLS (admitindo que os agregados são vesículas esféricas) e os medidos por DLS $\left(R_{e f}=d_{e f} / 2\right)$. Também sabemos que não existe reorganização das vesículas de DMPG, por exemplo através de fusão, (Lamy-Freund e Riske, 2003). Portanto, uma possível interpretação dos dados seria uma deformação anisotrópica dos agregados lipídicos. Por exemplo, as vesículas poderiam abrir-se formando planos fragmentados (Riske, Amaral, e Lamy, 2009; Barroso et al., 2010), e isso poderia levar ao aumento visto no raio de giração. Deste modo, medidas da anisotropia do centro espalhador poderiam nos ajudar a interpretar melhor os resultados.

Como vimos anteriormente, a polarizabilidade molecular é dada por um tensor. Para moléculas isotrópicas, a polarizabilidade molecular pode ser escrita como um escalar segundo a relação $(2.34)$

$$
\alpha=\frac{1}{3}\left(\alpha_{x x}+\alpha_{y y}+\alpha_{z z}\right)
$$

onde $\alpha_{x x}=\alpha_{y y}=\alpha_{z z}$.

O campo elétrico total é dado pela soma do campo externo e o campo molecular, como vimos na equação (2.99). O campo molecular é dado por

$$
\vec{E}=\frac{\vec{P}}{3 \epsilon_{0}}
$$

onde $\vec{P}$ é a polarização. Se assumirmos que todos os momentos de dipolo moleculares são iguais, a polarização pode ser escrita como vimos na equação (2.100)

$$
\vec{P}=\rho \vec{\mu}_{i}
$$

onde $\rho$ é a densidade de moléculas e $\vec{\mu}_{i}$ é o momento de dipolo molecular e pode ser escrito conforme a relação $(2.36)$

$$
\vec{\mu}_{i}=\epsilon_{0} \alpha \vec{E}_{i}
$$

Para moléculas anisotrópicas, temos, para uma dada molécula $i$, (conforme foi visto nas equações (2.35)):

$$
\begin{aligned}
& \mu_{x}=\epsilon_{0}\left(\alpha_{x x} E_{x}+\alpha_{x y} E_{y}+\alpha_{x z} E_{z}\right) \\
& \mu_{y}=\epsilon_{0}\left(\alpha_{y x} E_{x}+\alpha_{y y} E_{y}+\alpha_{y z} E_{z}\right) \\
& \mu_{z}=\epsilon_{0}\left(\alpha_{z x} E_{x}+\alpha_{z y} E_{y}+\alpha_{z z} E_{z}\right) .
\end{aligned}
$$


Logo, o campo elétrico total espalhado terá componentes nas direções $x, y$ e $z$, mesmo se a luz incidente for verticalmente polarizada.

Para verificar uma possível anisotropia do centro espalhador, acoplamos um polarizador em frente ao detector. O eixo de polarização do polarizador foi ajustado perpendicular à polarização do laser. Em primeiro lugar fizemos alguns controles. É conhecido da literatura que a razão de despolarização do benzeno é dada por

$$
\frac{I_{V H}}{I_{V V}}=0,26
$$

onde $I_{v h}$ é a intensidade da luz espalhada despolarizada na horizontal e $I_{v v}$ é a intensidade da luz espalhada polarizada na vertical, que é a direção da polarização da luz incidente. Esse controle foi satisfeito para todas as medidas de despolarização realizadas.

Ainda como controle medimos a razão de despolarização de micelas de SDS em água em $T=40^{\circ} C$, que apresentam pequena anisotropia e obtivemos razões de despolarização de aproximadamente 7,0\%. No trabalho, (Bergntröm e Pedersen, 1999), foi calculado os eixos do elipsoide $(\mathrm{a}, \mathrm{b}, \mathrm{c})$, das micelas formadas nas devidas concentrações $C=0,25 w t \%$ $(\sim 8,7 m M)$ com eixos $(\mathrm{a}=8,9 \mathrm{e} \mathrm{b}=\mathrm{c}=19,9) \AA, 0,50 w t \%(\sim 17,4 m M)$ com eixos $(\mathrm{a}=11,5$ e $\mathrm{b}=\mathrm{c}=19,8) \AA \mathrm{e} 1,0 w t \%(\sim 34,8 \mathrm{mM})$ com eixos $(\mathrm{a}=12,0$ e $\mathrm{b}=\mathrm{c}=20,4) \AA$. , (figura 6.25 à esquerda). Para esferas de poliestireno $(50 \mathrm{~nm})$, supostamente isotrópicas, obtivemos razões de despolarização inferiores a 1,0\%, e para o DMPC extrusado obtivemos razões da mesma ordem, conforme os resultados da figura 6.25.
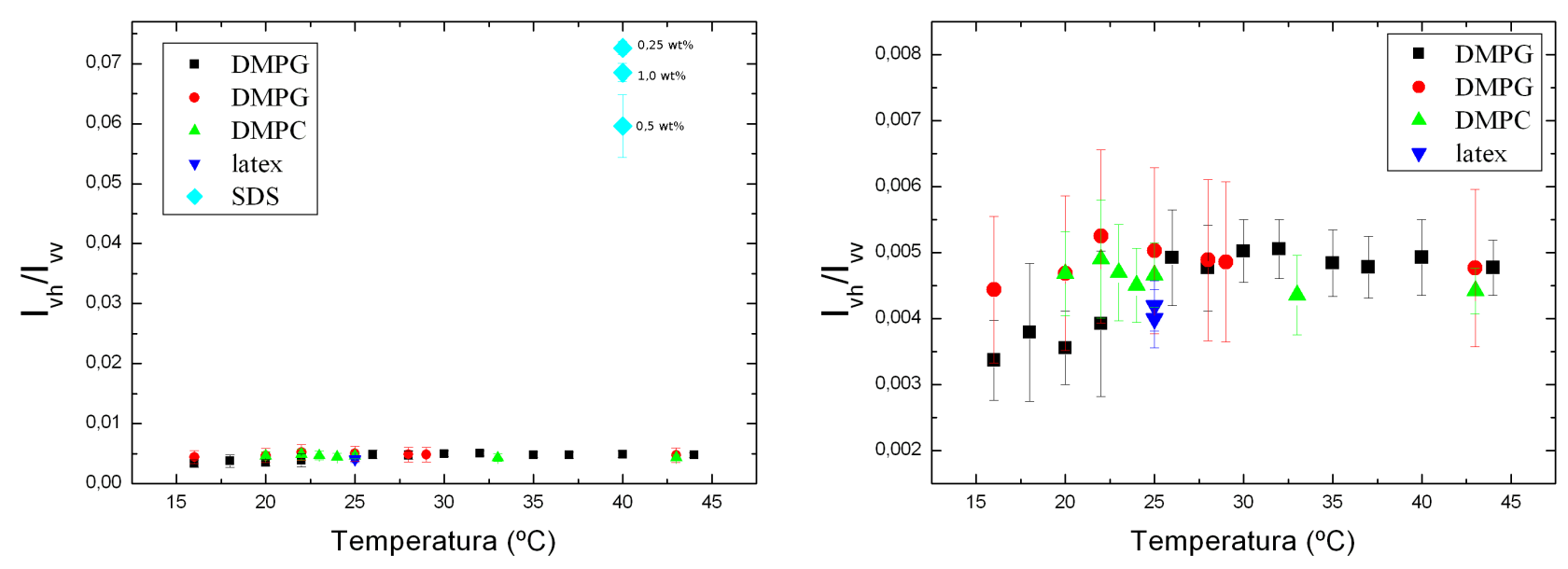

Figura 6.25: Medidas de anisotropia dos centros espalhadores. 
Como também podemos observar na figura 6.25, a razão de despolarização, ao longo da temperatura, para o DMPG extrusado apresenta valores semelhantes aos medidos para esferas de poliestireno (isotrópicas) e para o DMPC, que supostamente forma vesículas de geometria esférica. Na figura 6.25 (direita) não observamos nenhum aumento significativo (acima da flutuação dos erros) na região de transição, para as duas diferentes amostras analisadas.

Portanto, concluímos que o aumento observado no raio de giração na região de transição do DMPG não é causado por uma deformação anisotrópica, como por exemplo devido à abertura das vesículas.

\subsection{Discussões Gerais}

Os resultados obtidos por espalhamento de luz podem contribuir na compreensão do comportamento peculiar das dispersões de DMPG, em baixa força iônica. Como vimos anteriormente, há uma variedade de resultados experimentais e alguns modelos propostos que tentam explicá-los. Cada modelo contempla um conjunto de resultados. Um dos modelos propõe a formação de agregados de pequenas vesículas na fase gel e fluida, que desagregariam na região de transição, devido ao alto grau de dissociação dos lipídios, constatado experimentalmente (Riske et al., 1999; Barroso et al., 2010). Portanto, os lipídios estariam mais carregados nessa região, e a repulsão eletrostática entre eles seria a principal responsável pela desagregação. Esse modelo explicaria a queda na turbidez e o aumento da viscosidade, também observada nessa região, devido à formação de agregados menores. Entretanto, os resultados de espalhamento de luz estático nos mostram que o $R_{g}$ medido apresenta um grande aumento na região de transição, indicando um considerável aumento de tamanho, ou uma brusca mudança de forma. De acordo com os resultados de medidas de anisotropia do centro espalhador, o agregado lipídico não apresenta uma mudança estrutural anisotrópica, portanto, caso haja deformações do agregado na região de transição do DMPG, essas seriam isotrópicas. Os resultados de espalhamento de luz dinâmico também evidenciam um aumento de tamanho na região de transição, indicando que não há formação de agregados pequenos. Portanto, a brusca queda na turbidez deve ser atribuída à mudança nas propriedades ópticas da bicamada, semelhante, mas muito mais acentuada, à observada na transição da fase gel para a fase fluida (Disalvo, 1991).

Os resultados de SLS e DLS indicam que na região de transição do DMPG há agregados lipídicos de tamanhos efetivos maiores que os observados nas fases gel e fluida. Entretanto, 
os valores obtidos para o raio efetivo por esses métodos são bem discrepantes, considerando que a geometria esférica seja preservada. Como observamos também, o alto valor obtido para o $R_{g}$ não está relacionado a uma anisotropia de forma. Então, questionamos se esse aumento observado no raio efetivo medido por SLS poderia ser condizente com o outro modelo proposto na literatura, que sugere a formação de poros na região de transição do DMPG (Riske et al., 2009) ${ }^{3}$.

Antes de iniciarmos nossa discussão sobre a formação de poros, com os resultados obtidos por SLS, devemos fazer uma breve interrupção para chamar a atenção que não convém analisar os resultados de DLS com a proposta de vesículas perfuradas. Toda a discussão abordada aqui para DLS foi estruturada na relação de Stokes-Einstein, que admite esferas se difundindo em um fluído. Uma vesícula pode ser aproximada à uma esfera, visto que sua camada de hidratação é semelhante a de uma esfera. No entanto para objetos perfurados a relação de Stokes-Einstein, pode ser uma aproximação errada.

Já a análise por SLS independe da difusão, permeabilidade ou porosidade do centro espalhador, e está relacionada à interferência da luz espalhada por diferentes pontos do centro espalhador. Portanto, a intensidade da luz espalhada coletada a diversos ângulos possui informações sobre a forma das partículas espalhadoras. O $R_{g}$ pode ser visto como uma medida indireta do fator de forma, e precisamos relacioná-lo a alguma geometria conhecida para interpretar o resultado obtido por SLS, e é neste passo que entram os modelos propostos para a geometria do centro espalhador.

Então, admitindo que a vesícula seja preservada em uma forma esférica, podemos fazer um cálculo muito simples para estimar o número de poros na região de transição. Podemos calcular o número de lipídios necessários para formar um agregado lipídico de raio $R_{e f}$, dado pelas medias de espalhamento de luz em cada fase, ou na região de transição do DMPG.

O número de lipídios, $n$, necessários para formar uma vesícula de raio $R_{e f}$, pode ser estimado da seguinte forma

$$
n=\frac{4 \pi R_{e f}^{2}}{A_{C P L}}+\frac{4 \pi\left(R_{e f}-r\right)^{2}}{A_{C P L}},
$$

ou seja, a área da superfície externa da vesícula dividida pela área ocupada por cabeça polar de lipídio, $A_{C P L}$, nos fornece o número de lipídios da camada externa, (primeiro termo), somado a área da superfície interna da vesícula dividida por $A_{C P L}$, que equivale

\footnotetext{
${ }^{3} \mathrm{O}$ modelo proposto por Heimburg (Heimburg, 2007) não será discutido em nossas análises, uma vez que sua proposta sugere fusão dos agregados lipídicos na região de transição, e, como visto em (LamyFreund e Riske, 2003), não há troca de lipídios entre os agregados, indicando que não há fusão
} 
ao número de lipídios da camada interna, (segundo termo). Essa hipótese admite que, em média, os agregados lipídicos são do mesmo tamanho, e que as áreas ocupadas por lipídio na monocamada externa e interna são iguais.

Considerando que não ocorra fusão dos agregados lipídicos, como visto experimentalmente em (Lamy-Freund e Riske, 2003), então o número de lipídios em cada fase deve ser preservado constante. Tendo visto que o raio efetivo medido por SLS na região de transição é maior do que o observado na fase gel e fluida, podemos inferir que o número de lipídios em excesso será proporcional ao número de poros. Observe a figura 6.26.
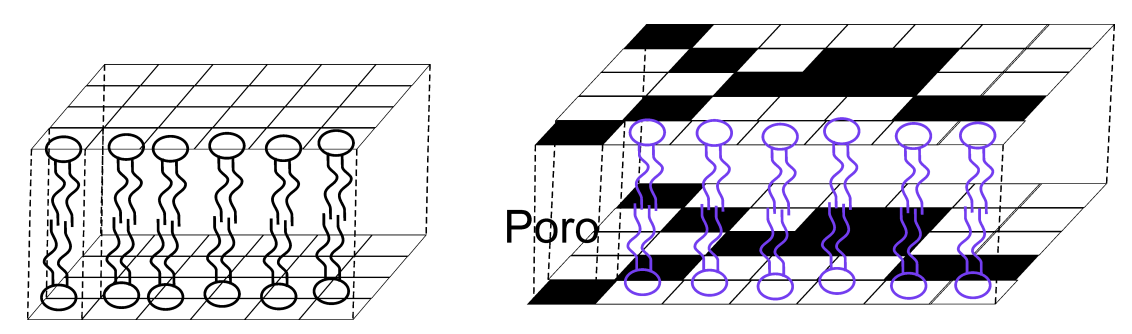

Figura 6.26: Ilustração do cálculo do número de lipídios e do número de poros. O número de lipídios é preservado constante na fase gel, fluida e na região de transição, portanto o número de lipídios em excesso será proporcional ao número de poros, dado pelos sítios em preto.

A figura ilustra uma parte da bicamada, onde os sítios brancos indicam a presença de lipídios, e os sítios pretos indicam sua ausência e portanto um poro. O tamanho de um pequeno poro será dado pelo mesmo tamanho do sítio ocupado por um lipídio, no entanto vários sítios pretos podem estar agrupados formando buracos maiores. Independente da disposição dos sítios pretos, podemos calcular a fração de área ocupada pelos poros.

Para esse cálculo utilizamos os valores resumidos na tabela 6.16 , onde os valores de $r$ foram extraídos de (Nagle e Tristram-Nagle, 2000; Marsh e Phil, 1990), para o DMPC. E os $R_{e f}$ foram obtidos a partir média dos valores experimentais da tabela 6.5.

Tabela 6.16: Valores utilizados para estimar o número de lipídios necessários para construir uma vesícula de $R_{e f}$.

\begin{tabular}{|ccc||c|}
\hline & $A_{C P L}\left(n m^{2}\right)$ & $r(n m)$ & $R_{e f}(n m)$ SLS \\
\hline \hline fase gel $\left(T=16^{\circ} C\right)$ & 0,48 & 4,42 & 31,1 \\
região de transição $\left(T=28^{\circ} C\right)$ & 0,60 & 3,55 & 89,0 \\
fase fluida $\left(T=43^{\circ} C\right)$ & 0,60 & 3,55 & 34,1 \\
\hline \hline
\end{tabular}

Esse cálculo leva a uma fração de área ocupada por poros de $86 \%$ da superfície externa ${ }^{4}$.

\footnotetext{
${ }^{4} \mathrm{~A}$ área ocupada por poros na superfície interna, pelo nosso cálculo assume valor $\sim 17 \%$ maior. No entanto, estamos considerando que a área ocupada por um lipídio na camada externa equivale a mesma área que na camada interna, o que superestima a fração de buracos na camada interna. Por isso, não
} 
Ainda como tentativa de obter frações de área menores para os poros, modificamos alguns parâmetros do cálculo realizado. Trabalhamos portanto com diferentes valores de $A_{C P L}$. O aumento da $A_{C P L}$ implica na diminuição do número de sítios, e os lipídios podem estar mais afastados entre si, o que pode ocorrer entre $T_{m}^{o n}$ e $T_{m}^{o f f}$. Podemos ver os resultados na tabela 6.17 .

\begin{tabular}{|c|c|c|}
\hline \multirow{6}{*}{ divera 0.14} & \multicolumn{2}{|c|}{$\begin{array}{r}\text { Porcentagem da área ocupada por buracos var } \\
\text { Área ocupada por buracos }\end{array}$} \\
\hline & $A_{C P L} n m^{2}$ & superfície externa \\
\hline & 0,70 & $79 \%$ \\
\hline & 0,80 & $78 \%$ \\
\hline & 0,90 & $76 \%$ \\
\hline & 1,00 & $74 \%$ \\
\hline
\end{tabular}

Mesmo aumentando a $A_{C P L}$ em mais de 50\%, a fração de área ocupada pelos poros ainda é muito grande, de acordo com os resultados obtidos por SLS. Nesse cálculo simples, não consideramos que parte dos lipídios estariam localizados no contorno do poro, visto que as cadeias hidrocarbônicas não ficariam diretamente expostas ao solvente. Essa consideração levaria a uma fração de área ocupada pelos poros ainda maior.

O grande aumento do raio de giração não condiz com a formação de poros na vesícula se esta for preservada em uma forma esférica. Portanto, sugerimos que o agregado lipídico deve apresentar algum tipo de deformação (isotrópica), que leva a esse aumento tão grande no raio de giração. A deformação não exclui a possibilidade da formação de poros.

Como discutimos anteriormente, o DMPG apresenta fatores favoráveis a mudanças estruturais na região de transição: cabeças polares carregadas e cadeias carbônicas curtas. O aumento da temperatura favoreceria a deformação, pois as cadeias hidrocarbônicas ganham mais liberdade e devido ao seu curto comprimento a interação hidrofóbica é menos intensa, favorecendo a deformação.

Deste modo, propomos um modelo para a região de transição do DMPG. Como discutimos na seção 6.1, figuras 6.1 e 6.3 à esquerda, vesículas pequenas apresentam a transição de fase gel-fluido alargada, possivelmente devido à diferença entre os empacotamentos das duas monocamadas lipídicas, interna e externa (Heimburg, 2007). Esse fato pode ser importante para vesículas de DMPG em baixa força iônica, pois vimos que nestas condições esse lipídio forma vesículas muito pequenas, nas fases gel e fluida encontramos um $R_{e f} \sim 30 \mathrm{~nm}$. Tendo o DMPG uma cabeça polar que pode ou não estar dissociada do vamos apresentar esse resultado. 
$\mathrm{Na}^{+}$, portanto carregada ou não, podemos supor que o grau de dissociação dos lipídios seja dependente do empacotamento da monocamada lipídica. Por exemplo, lipídios mais afastados (na monocamada externa) poderiam estar mais dissociados, portanto mais carregados. Então, ao ser aquecida, a bicamada seria deformada, pela coexistência de regiões onde a repulsão eletrotática entre as cabeças polares favoreceria a existência de regiões muito frouxas, e regiões menos carregadas, portanto mais estruturadas. Isso poderia levar a grandes deformações da bicamada lipídica e da vesícula de DMPG (figura 6.27). Além disso, considerando os altos valores da capacidade térmica entre $T_{m}^{o n}$ e $T_{m}^{o f f}$, indicativos de grandes flutuações de energia e densidade, é razoável supor que devemos ter grandes flutuações da superfície da vesícula.

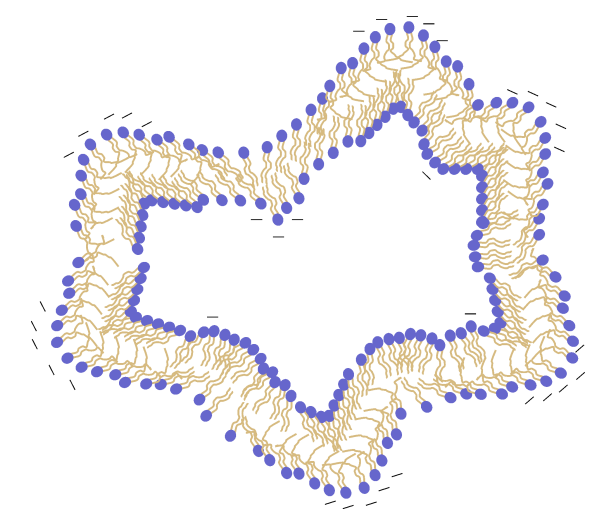

Figura 6.27: Modelo da deformação do DMPG, na região de transição.

O modelo proposto também estaria de acordo com os resultados experimentais observados na literatura, para a região de transição do DMPG:

- Altos valores da capacidade térmica entre $T_{m}^{o n}$ e $T_{m}^{o f f}$, (Riske et al., 2001), indicam grandes flutuações de energia e densidade da bicamada lipídica. Nessa região há um balanço entre interações: interação hidrofóbica dada pelas cadeias hidrocarbônicas (atrativa), e interações eletrostáticas entre as cabeças polares carregadas (repulsiva). As deformações propostas ao agregado lipídico apresentam a competição constante entre esses dois fatores, visto que há regiões em que os lipídios estão mais próximos e outras em que estão mais distantes.

- Queda brusca da turbidez (intensidade da luz espalhada), perda do contraste de fase observado pela microcopia óptica, (Riske et al., 2004, 2009). A bicamada encontraria-se mais hidratada nesta região, e muito mais permeável ao solvente. Deste modo, a diferença entre o índice de refração da água e bicamada é reduzida, levando à queda na turbidez e perda do contraste óptico. 
- Observação de fragmentos de bicamada lipídica com grandes flutuações por microscopia de fluorescência, (Riske et al., 2009). Os fragmentos observados com sondas fluorescente podem estar relacionados com as deformações/flutuações das vesículas.

- Coexistência de duas regiões com diferentes curvaturas, vista por RPE (Riske et al., 2003). Como proposto no modelo, o agregado lipídico deformado apresentaria: regiões mais carregadas e com maior repulsão entre as cabeças polares, portanto de curvatura maior, tipo micela, e regiões menos carregadas, que poderiam estar mais próximas e portanto com menor curvatura.

- Não há fusão dos agregados lipídicos ao longo da transição de fase (Lamy-Freund e Riske, 2003).

- Aumento da condutividade entre $T_{m}^{o n}$ e $T_{m}^{o f f}$ devido ao maior grau de dissociação das cabeças polares, (Riske et al., 1997; Barroso et al., 2010), que também está relacionado ao aumento de $A_{2}$. A alta dissociação das cabeças polares sugere que a camada externa apresentará muitos lipídios carregados, os lipídios mais carregados favorecem as curvaturas maiores. Voltaremos a essa discussão adiante.

- Aumento da viscosidade (Riske et al., 1997; Barroso et al., 2010). As deformações e as grandes flutuações aumentariam a macro viscosidade do meio.

- Pico de repetição observado no SAXS (Riske et al., 2004), pode estar relacionado à distância entre os agregados lipídicos, só evidente na transição de fase devido às grandes deformações da vesícula. No entanto, é necessário mais investigações e cálculos.

Como vimos, a contribuição eletrostática exerce forte influência no modelo proposto, pois, como já apresentado, o aumento da foça iônica reduz a região de transição, (Riske et al., 1997). No trabalho (Barroso et al., 2010) foi discutido que o aumento da concentração de DMPG também reduz a região de transição: a dissociação do DMPG levaria a um aumento da concentração de contra-íons em solução. Portanto, um dos fatores determinantes da extensão da região de transição deve estar relacionado ao coeficiente de dissociação, $\alpha$, das cabeças polares do DMPG, (Barroso et al., 2010). Neste trabalho, foi feito um cálculo muito simples explicando a dependência da ionização com a concentração de DMPG. Neste, foi levado em conta a concentração de contra-íons em solução. 
Representamos abaixo uma discussão adicional, sobre a possível diferença de ionização das monocamadas interna e externa, baseada no cálculo discutido em (Barroso et al., 2010).

Mesmo sabendo que os contra-íons distribuem-se com probabilidades diferentes ao longo da distância à superfície de uma bicamada carregada, podemos calcular uma concentração média de contra-íons em solução (no volume externo à vesícula). Essa concentração será dada pela fração de lipídios dissociados na bicamada externa de todas as vesículas, dividido pelo volume da solução, excluído o volume ocupado pelas vesículas, conforme a equação (6.2)

$$
[\text { contra }- \text { ions }]_{e x t}(M)=\alpha \frac{n N_{v e s}}{2 V_{T}(1-\phi)} \frac{1}{N_{A v}}
$$

onde $n$ é o número de lipídios por vesícula, $N_{\text {ves }}$ é o número de vesículas, $V_{T}$ é o volume total da solução e $\phi$ é a fração de volume ocupado pelas vesículas. Também podemos observar que

$$
\frac{N_{\text {ves }}}{V_{T}}=\frac{[D M P G] N_{A v}}{n}
$$

$\log$,

$$
[\text { contra }- \text { ions }]_{e x t}(M)=\alpha \frac{[D M P G]}{2(1-\phi)}
$$

Podemos calcular também a concentração de contra-íons no interior da vesícula, que será dada pela fração de lipídios dissociados da camada interna, $\alpha^{\prime}$, dividida pelo volume interno da vesícula, $V_{\text {int }} \sim V_{\text {vesicula }}$, conforme a equação (6.5),

$$
[\text { contra }- \text { ions }]_{\text {int }}(M)=\alpha^{\prime} \frac{n}{2 V_{\text {int }}} \frac{1}{N_{A v}},
$$

onde

$$
V_{\text {int }}=\frac{4}{3} \pi R_{\text {ef }}^{3}, \quad \frac{n}{2}=\frac{4 \pi R_{e f}^{2}}{A_{C P L}},
$$

portanto

$$
[\text { contra }- \text { ions }]_{\text {int }}(M)=\frac{3 \alpha^{\prime}}{N_{A v} A_{C P L} R} .
$$

Dada as equações (6.2) e (6.5) e substituindo os valores de $A_{C P L}$ e do raio $R$, obtemos os valores, listados na tabela 6.18. Utilizamos o valor $A_{C P L}=0,60 \mathrm{~nm}^{2}$ (Nagle e TristramNagle, 2000; Marsh e Phil, 1990). Este valor corresponde a área ocupada por lipídio na fase fluida, medido para o DMPC. Escolhemos tal parâmetro pois esse corresponde a maior área ocupada por lipídio observada, e sugerimos que pelo fato do DMPG ser muito permeável ao solvente, os seus lipídios ocupem uma área por cabeça polar relativamente grande. Estimamos também um valor médio para o raio efetivo do DMPG, $R=30 \mathrm{~nm}$, na região de transição. 
Tabela 6.18: Concentração de contra-íons no volume externo às vesículas e no volume interno das vesículas.

\begin{tabular}{|c||ccc|}
\hline$[\mathrm{DMPG}]$ & $1,0 \mathrm{mM}$ & $10,0 \mathrm{mM}$ & $50,0 \mathrm{mM}$ \\
\hline \hline$[\text { contra }- \text { ions }]_{\text {ext }}$ & $\alpha 5,010^{-4}(M)$ & $\alpha 5,110^{-3}(M)$ & $\alpha 2,710^{-2}(M)$ \\
{$[\text { contra }- \text { ions }]_{\text {int }}$} & $\alpha^{\prime} 0,17(M)$ & $\alpha^{\prime} 0,17(M)$ & $\alpha^{\prime} 0,17(M)$ \\
\hline \hline
\end{tabular}

Podemos assumir que a concentração de contra-íons no volume externo à vesícula seja igual ou semelhante à do volume interno da vesícula, caso contrário a variação de pressão osmótica (com relação aos contra-íons) poderia romper a membrana.

Então, verificamos dos resultados da tabela 6.18 que o grau de dissociação da cabeça polar dos lipídios deve ser diferente na camada externa e na camada interna, para que a concentração de contra-íons seja mantida aproximadamente constante interna e externamente. Notamos também que $\alpha>>\alpha^{\prime}$, e portanto a camada externa deveria estar muito mais carregada que a camada interna. Essa diferença no grau de ionização das camadas interna e externa pode favorecer as deformações. Como vimos a camada externa pode estar mais carregada, e portanto com maior probabilidade de ter regiões com curvaturas maiores, onde os lipídios encontram-se mais afastados, nessas regiões, devido a repulsão eletrostática. Por outro lado, na camada interna onde o grau de ionização seria menor, as cabeças polares poderiam estar mais próximas.

Como perspectiva futura propomos calcular numericamente o raio de giração de um objeto deformado, semelhante ao ilustrado na figura 6.27. O modelo qualitativo proposto ainda é muito simples. Certamente há a necessidade de um modelo estatístico, que aborde as propriedades termodinâmicas do sistema. 



\section{Capítulo 7}

\section{Conclusões}

Observamos que dispersões lipídicas de DMPG podem apresentar uma grande população de agregados de tamanhos pequenos. Pois, quando extrusamos a dispersão lipídica por um filtro de poro de $100 \mathrm{~nm}$, obtemos vesículas com diâmetro efetivo da ordem de 50 - $60 \mathrm{~nm}$. Como foi constatdo o DMPG extrusado é constituído por vesículas com grandes curvaturas.

Na região de transição do DMPG, observamos dos resultados de SLS que os valores de $A_{2}$ indicam aumento das interações repulsivas entre os agregados. Isto está de acordo com o alto grau de dissociação das cabeças polares, já observado nesta região, e portanto aumento da repulsão eletrostática das cabeças. Além disso, também observamos que o $R_{g}$, nesta região, apresenta um aumento de aproximadamente três vezes o valor obtido para a fase gel. Investigamos se o grande aumento do raio de giração poderia ser devido à alguma anisotropia de forma. Entretanto, medidas de anisotropia do centro espalhador mostram que as vesículas lipídicas não apresentam deformações anisotrópicas.

Os resultados observados por DLS também evidenciaram um aumento do raio efetivo na região de transição. No entanto, o aumento observado da fase gel para a região de transição corresponde a aproximadamente 28\%, em média. Deste modo, o raio efetivo calculado de acordo com os resultados dos diferentes métodos, SLS e DLS, admitindo que os agregados lipídicos sejam vesículas esféricas, são muito diferentes entre si. Sendo o raio efetivo obtido por SLS maior que o obtido por DLS, $R_{e f}^{S L S}>R_{e f}^{D L S}$. Sugerimos que o DMPG seja muito permeável ao solvente, na região de transição, explicando então a brusca queda na intensidade da luz espalhada. Esse fato pode dificultar medidas do coeficiente de difusão, e pode levar a tamanhos efetivos aparentemente menores. Por outro lado, as medidas de SLS nos levaram a tamanhos efetivos superestimados segundo a geometria que utilizamos (casca esférica). Como perspectiva futura, convém analisar 
outras geometrias, que abrangem deformações, e flutuações para compreender melhor a forma do agregado lipídico nesta região.

Com esses resultados propomos um novo modelo para a região de transição. Supomos que o agregado lipídico apresente deformações isotrópicas com grandes flutuações de forma, e a coexistência de regiões de diferentes curvaturas, como já observado na literatura. Propomos que a região de maior curvatura esteja mais carregada, e portanto as cabeças lipídicas podem estar mais afastadas. Esta região seria encontrada preferencialmente na camada externa. Por outro lado, na camada interna, observaríamos regiões com curvaturas menores e menos carregada. Sugerimos esse modelo, pois observamos que a camada externa pode estar muito mais carregada que a interna, devido à diferença do grau de dissociação das cabeças polares nas camadas externa e interna. O modelo proposto também explicaria os demais resultados experimentais apresentados na literatura.

Concluímos também que a combinação de medidas de SLS e DLS permite fazer avaliações mais completas do sistema. 


\section{Apêndice A}

\section{Expansão dos Cumulantes}

\section{Introdução}

Neste apêndice vamos demonstrar como a função de correlação do campo elétrico $g^{(1)}(\tau)$ pode ser escrita em termos de expanão em cumulanttes, que por sua vez pode ser escrito em termos dos momentos de uma distribuição. O estudo e a compreensão desse método foi realizado em colaboração com a $\operatorname{Prof}^{a} \operatorname{Dr}^{a}$ Carla Goldman.

A função de correlação do campo elétrico pode ser dada por uma distribuição de taxas de decaimentos $\Gamma$, logo podemos escrever,

$$
g^{(1)}(\tau)=\int P(\Gamma) \exp (-\Gamma \tau) d \Gamma=\mu(\tau) .
$$

Definimos a função geratriz dos momentos

$$
\mu(\tau)=\int_{0}^{\infty} P(\Gamma) \exp (-\Gamma \tau) d \Gamma,
$$

que corresponde à transformada de Laplace de $P(\Gamma)$. Podemos expandir a exponencial na equação (A.2), de forma a escrever

$$
\begin{aligned}
\mu(\tau) & =\int_{0}^{\infty} P(\Gamma)\left[1-\Gamma \tau+\frac{1}{2} \Gamma^{2} \tau^{2}+\cdots\right] d \Gamma \\
& =\sum_{n=0}^{\infty} \int_{0}^{\infty} d \Gamma P(\Gamma) \frac{(-\Gamma \tau)^{n}}{n !} \\
& =\sum_{n=0}^{\infty} \frac{(-\tau)^{n}}{n !} \int_{0}^{\infty} d \Gamma P(\Gamma) \Gamma^{n}
\end{aligned}
$$

onde $\int \Gamma^{n} P(\Gamma) d \Gamma \equiv M_{n}$ corresponde ao momento de ordem $n$ da distribuição, isto é, são os valores esperados das potências das variáveis aleatórias:

$$
\langle\Gamma\rangle=\int \Gamma P(\Gamma) d \Gamma, \quad\left\langle\Gamma^{2}\right\rangle=\int \Gamma^{2} P(\Gamma) d \Gamma, \quad \cdots .
$$


Portanto,

$$
\mu(\tau)=\sum_{n=0}^{\infty} \frac{(-\tau)^{n}}{n !} M_{n}
$$

ou ainda, separando o termo de ordem zero,

$$
\mu(\tau)=1+\sum_{n=1}^{\infty} \frac{(-\tau)^{n}}{n !} M_{n}
$$

pois $\int \Gamma^{0} P(\Gamma) d \Gamma=1$, uma vez que a distribuição é normalizada. Podemos definir a função geratriz dos cumulantes, $\mathcal{K}(\tau)$, segundo a equação

$$
\mathcal{K}(\tau) \equiv \ln [\mu(\tau)]
$$

de forma que

$$
\begin{aligned}
\mathcal{K}(\tau) & =\ln \left[\sum_{n=0}^{\infty} \frac{(-\tau)^{n}}{n !} M_{n}\right] \\
& =\sum_{n=0}^{\infty} \frac{(-\tau)^{n}}{n !} K_{n}
\end{aligned}
$$

onde $K_{n}$ é o cumulante de ordem $n$. Logo

$$
\mu(\tau)=\exp \left[\sum_{n=0}^{\infty} \frac{(-\tau)^{n}}{n !} K_{n}\right]
$$

De acordo com as equações (A.1) e (A.6),

$$
\begin{aligned}
\mathcal{K}(\tau) & =\ln [\mu(\tau)]=\ln \left[1+\sum_{n=1}^{\infty} \frac{(-\tau)^{n}}{n !} M_{n}\right] \\
& =\ln (1+\epsilon)
\end{aligned}
$$

de forma que, expandindo $\ln (1+\epsilon)$, temos

$$
\ln (1+\epsilon)=\epsilon-\frac{\epsilon^{2}}{2}+\frac{\epsilon^{3}}{3}+\cdots
$$

$\log$,

$$
\mathcal{K}(\tau)=\sum_{n=1}^{\infty} \frac{(-\tau)^{n}}{n !} M_{n}-\frac{1}{2} \sum_{n=1}^{\infty} \sum_{m=1}^{\infty} \frac{(-\tau)^{n+m}}{n ! m !} M_{n} M_{m}+\cdots
$$

Vamos considerar apenas os termos de ordem inferior a três. Assim,

$$
\begin{aligned}
\mathcal{K}(\tau) & =-\tau M_{1}+\frac{\tau^{2}}{2} M_{2}-\frac{1}{2} \tau^{2} M_{1} M_{1} \\
& =-\tau\langle\Gamma\rangle+\frac{\tau^{2}}{2}\left\langle\Gamma^{2}\right\rangle-\frac{\tau^{2}}{2}\langle\Gamma\rangle^{2} \\
& =-\tau\langle\Gamma\rangle+\frac{\tau^{2}}{2} \sigma^{2}
\end{aligned}
$$


onde $\sigma^{2}$ é a variância da distribuição $P(\Gamma)$, e está relacionada ao índice de polidispersão. Visto que $\mu(\tau)=\exp [\mathcal{K}(\tau)]$, de acordo com as equações (A.7) e (A.1), temos

$$
g^{(1)}(\tau)=\exp \left(-\tau\langle\Gamma\rangle+\frac{\tau^{2}}{2} \sigma^{2}\right) .
$$

Ou seja, a função de correlação do campo elétrico $g^{(1)}(\tau)$ pode ser ajustada por um termo de decaimento médio, levando em conta também a dispersão da distribuição.

Um outro método utilizado na análise dos resultados, chamado Contin, parte do mesmo princípio. A função de autocorrelação do campo elétrico é dada pela equação (A.1). Por meio de uma transformada inversa de Laplace é possível determinar numericamente $P(\Gamma)$. Esses métodos foram comparados para garantir que o resultado experimental não dependa da forma de análise.

Para a avaliação e comparação dos resultados, focamos as análises nos valores médios obtidos a partir de ambos os métodos. 



\section{Apêndice B}

\section{Análise da função de auto correlação utilizando o programa Gendist}

Outro método também foi usado para fazer essas análises de espalhamento de luz dinâmico, utilizando o programa Gendist, (Jakes, 1995). Neste, a função de auto correlação do campo elétrico normalizada, obtida a partir de $g^{(2)}\left(\tau_{r}\right)$, é escrita em termos de uma distribuição de tempos de relaxação, $A\left(\tau_{r}\right)$,

$$
g^{(2)}\left(\tau_{r}\right)-1=\beta\left(\int A\left(\tau_{r}\right) \exp \left(-t / \tau_{r}\right) d \tau_{r}\right),
$$

onde $\tau_{r}$ corresponde ao tempo de relaxação da difusão, $t$ corresponde ao "delay time" e $\beta$ é um parâmetro experimental, relacionado à linha de base.

Mostramos como exemplo apenas alguns ajustes referentes a concentração $C=0,34 \mathrm{mM}$ de DMPG, na figura B.1.
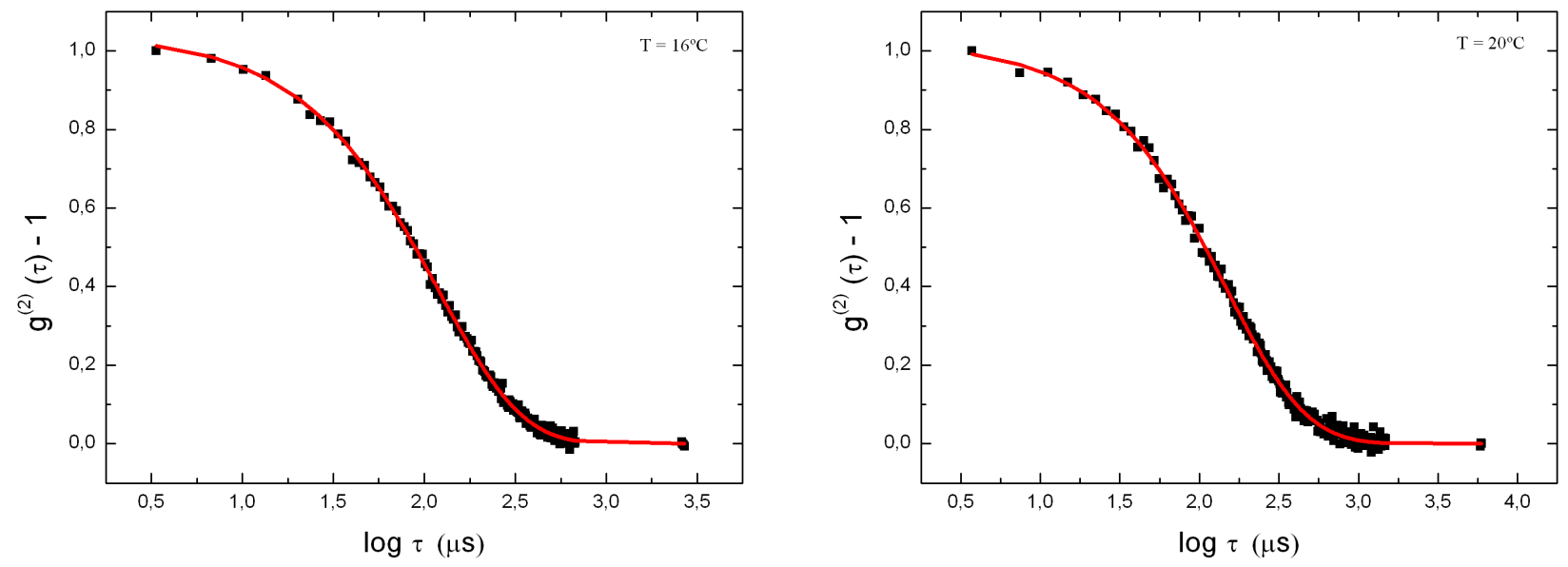
APÊNDICE B. ANÁLISE DA FUNÇÃO DE AUTO CORRELAÇÃo UTILIZANDO O
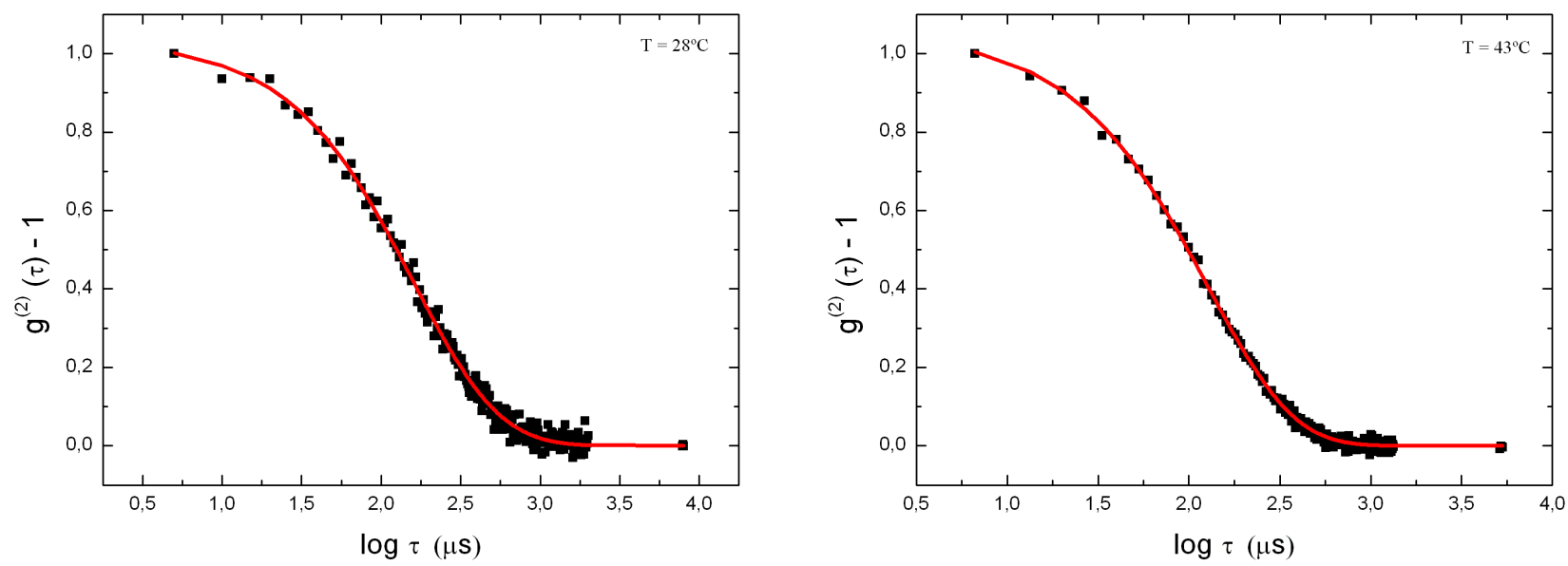

Figura B.1: Exemplo das função de correlação do campo elétrico e o ajuste obtido pelo método do programa Gendist, para o DMPG, onde $\tau$ é dado em $\mu s$.

Podemos observar as distribuições de tamanhos obtidas pelas análises anteriores, na figura B.2, e os valores dos diâmetro efetivos médios estão resumidos na tabela B.1 e podem ser comparados com os valores obtidos para a mesma concentração utilizando o método dos Cumulantes para essa mesma concentração. Ambos os métodos fornecem um valor de z-average.

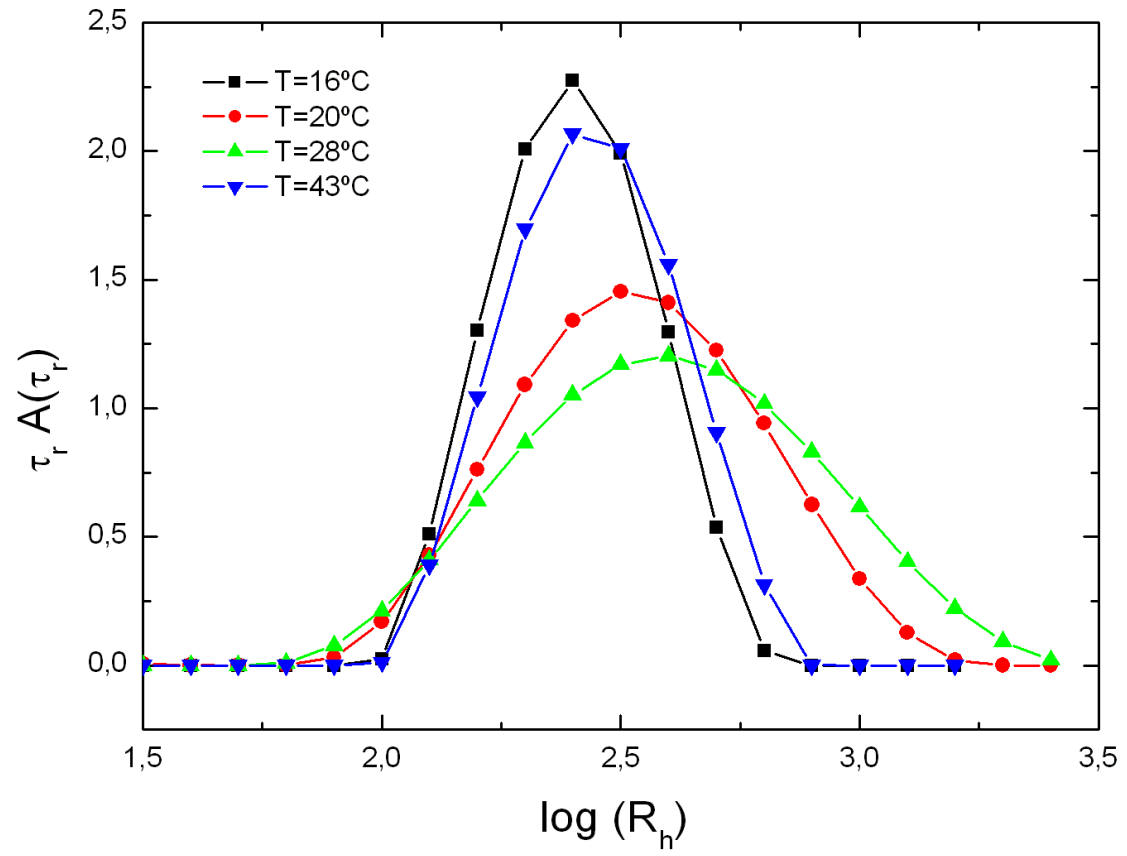

Figura B.2: Distribuição de tamanhos obtida pelo método do programa Gendist, DMPG $C=0,34 m M$. 
Tabela B.1: Comparação dos métodos Cumulantes segunda ordem e Gendist, para o DMPG $C=0,34 \mathrm{mM}$.

\begin{tabular}{|cc|c|}
\hline Temperatura ${ }^{\circ} \mathrm{C}$ & Cumulantes $d_{\text {ef }}(\mathrm{nm})$ & Gendist $d_{\text {ef }}(\mathrm{nm})$ \\
\hline \hline 16 & $50 \pm 2$ & $47 \pm 1$ \\
20 & $58 \pm 2$ & $58 \pm 1$ \\
28 & $62 \pm 2$ & $63 \pm 1$ \\
43 & $52 \pm 2$ & $53 \pm 1$ \\
\hline \hline
\end{tabular}

Observamos excelente concordância dos resultados com os diferentes métodos de análise.

Na figura B.2 podemos notar que na região de transição do DMPG, o valor médio da distribuição é deslocado para tamanhos maiores e a largura da distribuição aumenta, indicando um aumento do índice de polidispersão do DMPG nesta região. A fase gel e fluida apresentam valores do índice de polidispersão semelhantes. Também obtemos o índice de polidispersão pelo método dos Cumulantes, que concorda com o resultado anterior, conforme vimos na tabela 6.9.

O mesmo tratamento de dados foi realizado para o DMPC. Comparamos as análises obtidas pelo método dos Cumulantes com o método do programa Gendist. Podemos observar alguns exemplos do ajuste da função de autocorrelação do campo elétrico normalizada na figura B.3, e as distribuições na figura B.4, para a solução mais diluída de DMPC, $C=0,49 \mathrm{mM}$.
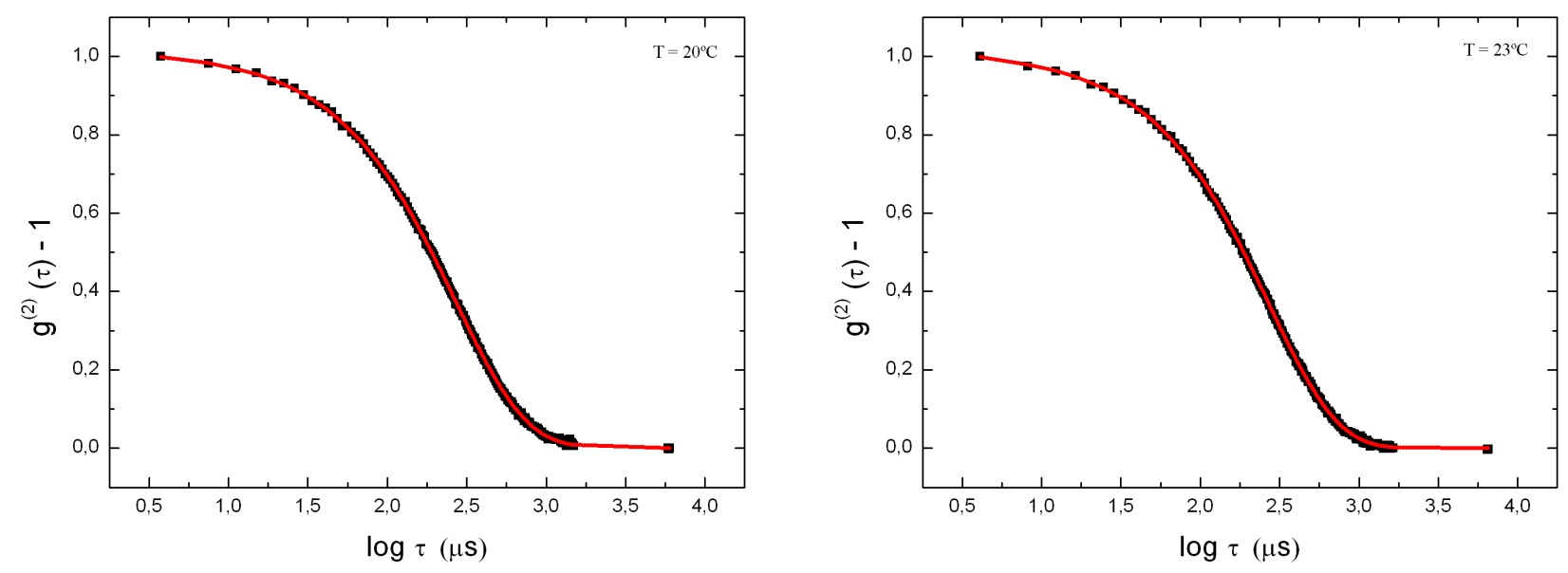

Os resultados das análises apresentadas como exemplo, da figura B.4, podem ser resumidas e comparadas com as respectivas análises obtidas pelo método dos Cumulantes de segunda ordem, na tabela B.2.

Mesmo apresentando diferenças maiores para DMPC, os dois métodos Cumulantes e Gendist, ainda apresentam uma boa concordância nos resultados. 


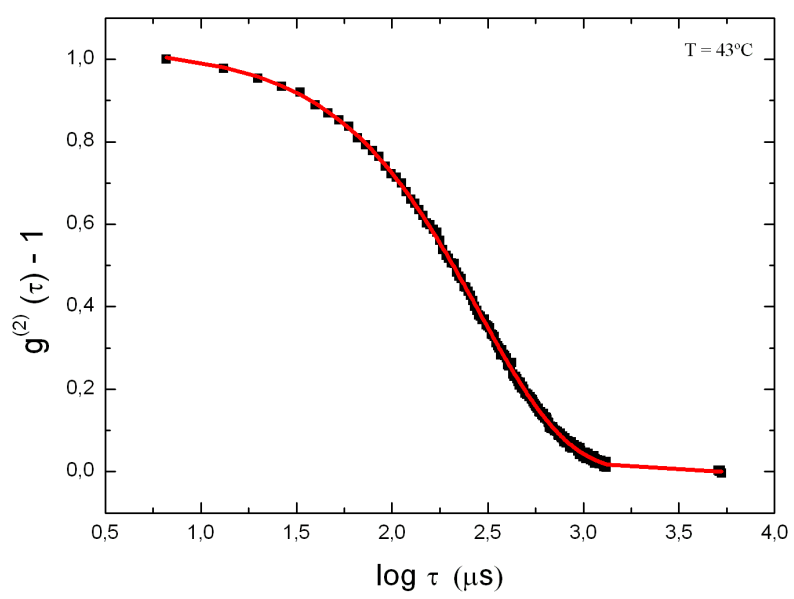

Figura B.3: Exemplo das função de correlação do campo elétrico e o ajuste obtido pelo método do programa Gendist, para o DMPC, onde $\tau$ é dado em $\mu s$.

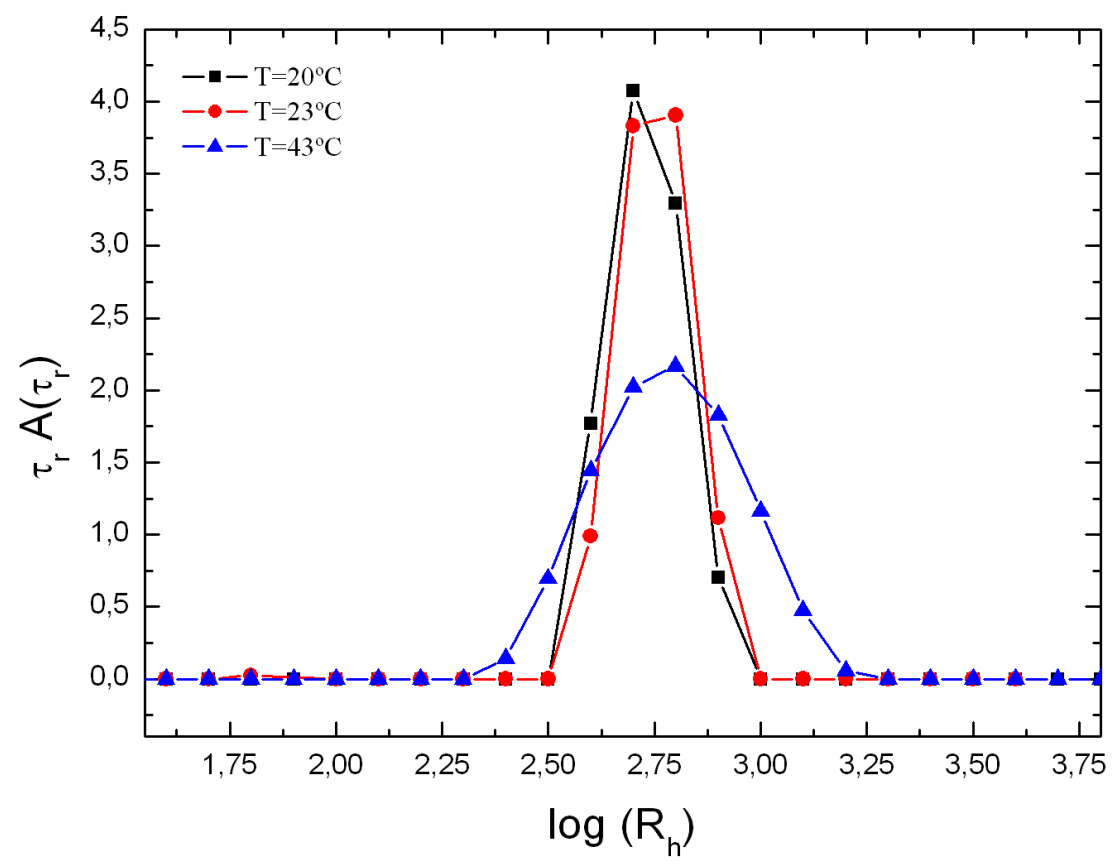

Figura B.4: Distribuição de tamanhos obtida pelo método do programa Gendist, DMPC $C=0,49 m M$.

Tabela B.2: Comparação dos métodos Cumulantes ( $2^{a}$ ordem) e Gendist, para o DMPC $C=0,49 m M$.

\begin{tabular}{|cc|c|}
\hline Temperatura ${ }^{\circ} C$ & Cumulante $d_{\text {ef }}(\mathrm{nm})$ & Gendist $d_{\text {ef }}(\mathrm{nm})$ \\
\hline \hline 20 & $99 \pm 1$ & $104 \pm 2$ \\
23 & $99,5 \pm 0,2$ & $107 \pm 2$ \\
43 & $112,0 \pm 0,4$ & $113 \pm 1$ \\
\hline
\end{tabular}


Embora neste apêndice mostramos apenas dois exemplos, o método obtido pelo programa Gendist foi aplicado para todas as curvas de correlação obtidas e discutidas nesta dissertação. Para todas elas houve uma boa concordância dos resultados, quando comparamos os diferentes métodos, conforme ilustrado nos exemplos. Testamos esse outro método (Gendist) para avaliar a confiabilidade das análise, e supomos, devido a concordância dos dois métodos, que todas as análises são confiáveis. 



\section{Apêndice $\mathrm{C}$}

\section{Comparação do DMPG não}

\section{extrusado e extrusado por DLS}

Neste apêndice vamos mostrar o efeito da extrusão no DMPG, e seus interessantes resultados. Este processo foi adotado para reduzir o efeito da índice de polidispersão das dispersões lipídicas.

Curiosamente, observamos por DLS que o DMPG não extrusado apresentava diâmetros efetivos da ordem de $120 \mathrm{~nm}$ e que o DMPG extrusado apresentava valores bem menores, da ordem de $60 \mathrm{~nm}$. Essa constatação foi surpreendente pois o DMPG pode ser muito polidisperso, sendo composto por diferentes populações de vesículas de tamanhos grandes e pequenos, que quando extrusado as vesículas grandes deixam de compor a amostra e passamos a olhar para uma solução de índice de polidispersão menor. Outra hipótese seria que após a extrusão há a formação de agregados menores. No entanto, o mais surpreende desta comparação foi observar que na região de transição do DMPG não extrusado os resultados DLS indicam uma diminuição no tamanho do agregado lipídico, e inclusive esse resultado já tinha sido observado na literatura, (Alakoskela e Kinnunnen, 2007). Por outro lado, para o DMPG extrusado, observamos um aumento de tamanho na região de transição. Observe a figura C.1. 


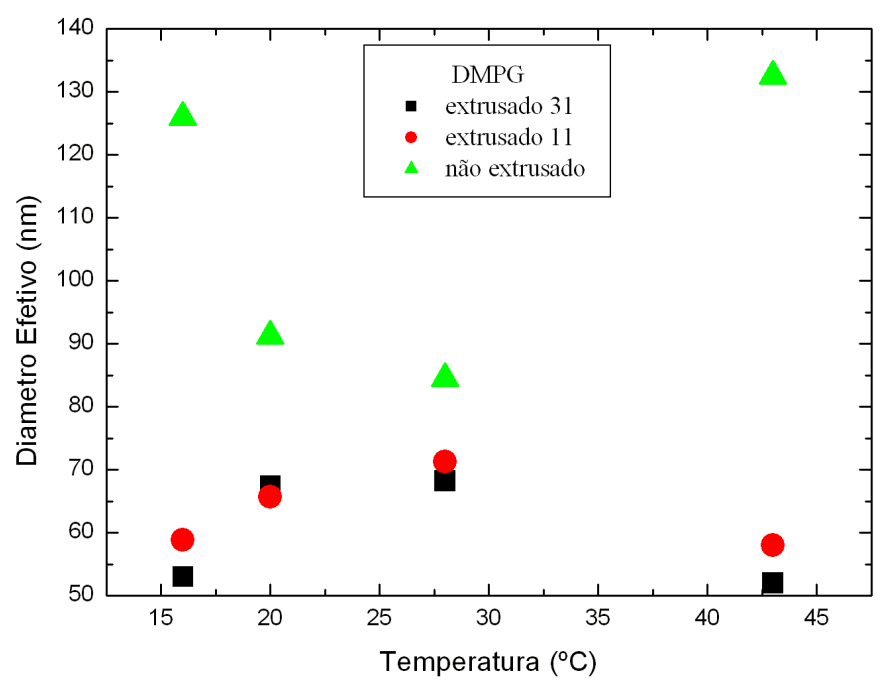

Figura C.1: Comparação do diâmetro efetivo do DMPG não extrusado com o DMPG extrusado (11 e 31 vezes), obtidos por DLS.

Os resultados apresentados no gráfico anterior C.1 foram análisados pelo método dos Cumulantes de segunda ordem. As medidas de DLS foram realizadas em diversos ângulos de espalhamento 50, 70, 90, 110 e $120^{\circ}$, e coeficiente de difusão foi determinado pelo coeficiente angular da reta $\Gamma=D_{T} q^{2}$ (no gráfico $\left.\Gamma \times q^{2}\right)$, em seguida o diâmetro efetivo foi determinado pela equação de Stokes-Einstei 3.15. A medida foi realizada para amostras não extrusadas, extrusadas 11 vezes e extrusadas 31 vezes, como apresentado anteriormente na seção 6.3. Os resutados do gráfico anterior foram resumidos na tabela C.1.

Devemos lembrar que a técnica de espalhamento de luz fornece um diâmetro efetivo médio, pesado pela massa ao quadrado, conhecido com $z_{a v}$

$$
d_{e f}=\frac{\sum_{i} N_{i} M_{i}^{2} d_{i}}{\sum_{i} N_{i} M_{i}^{2}} .
$$

Então, quando a dispersão é composta por um número pequeno de partículas grandes, a média obtida para o diâmetro efetivo estará deslocada para valores maiores, visto que o peso atribuído aos tamanhos grandes será muito maior.

Podemos mostrar um exemplo obtido pela aluna de iniciação, Nomura, D. A., com esferas de poliestireno de diferentes tamanhos, observe a tabela C.2

Podemos observar também que conforme extrusamos mais vezes o DMPG, obtemos um diâmetro efetivo menor. Observamos primeiro, quando extrusamos 11 vezes o diâmetro efetivo medido diminui aproximadamente pela metade. Nesse processo de extrusão devemos estar eliminando os agregados grandes que deslocam o $z_{a v}$ para valores maiores. De- 
Tabela C.1: Diâmetro efetivo do DMPG após diferentes números de extrusões.

\begin{tabular}{|c|c|c|}
\hline Temperatura ${ }^{\circ} \mathrm{C}$ & Número de extrusões & $d_{\text {ef }}(\mathrm{nm})$ \\
\hline \hline \multirow{2}{*}{16} & 0 & 126 \\
& 11 & 59 \\
& 31 & 53 \\
\hline \hline \multirow{2}{*}{20} & 0 & 91 \\
& 11 & 66 \\
& 31 & 67 \\
\hline \hline \multirow{2}{*}{28} & 0 & 85 \\
& 11 & 71 \\
& 31 & 68 \\
\hline \hline \multirow{2}{*}{43} & 0 & 133 \\
& 11 & 59 \\
& 31 & 52 \\
\hline \hline
\end{tabular}

Tabela C.2: Diâmetro efetivo de misturas de esferas de poliestireno de diferentes tamanhos, (Nomura, Enoki e Lamy - trabalho em fase de conclusão).

\begin{tabular}{|c|c|c|}
\hline Tamanhos $(20: 90)(\mathrm{nm})$ proporção em número & $d_{e f}(\mathrm{~nm})\left(z_{a v}-\right.$ calculado $)$ & $d_{e f}(\mathrm{~nm})\left(z_{a v}-\right.$ medido $)$ \\
\hline \hline $700: 1$ & 86 & $80 \pm 1$ \\
$1400: 1$ & 80 & $72 \pm 4$ \\
$2100: 1$ & 76 & $69 \pm 1$ \\
\hline \hline Tamanhos $(50: 90)(n m)$ proporção em número & $d_{\text {ef }}(\mathrm{nm})\left(z_{a v}\right.$ - calculado $)$ & $d_{\text {ef }}(\mathrm{nm})\left(z_{a v}-\right.$ medido $)$ \\
\hline \hline $9: 1$ & 86 & $76 \pm 1$ \\
$45: 1$ & 73 & $67,1 \pm 0,3$ \\
$135: 1$ & 61 & $55 \pm 1$ \\
\hline \hline
\end{tabular}

pois quando extrusamos 31 vezes ainda observamos uma pequena diminuição, novamente, podemos estar eliminando as poucas partículas grandes que eventualmente restaram.

Tendo em vista que o movimento browniano seja produzido pelas colisões das pequenas partículas do fluido, podemos escrever a equação de Langevin da seguinte forma,

$$
m \frac{d v}{d t}=-\gamma v+F_{a}(t)
$$

onde $\gamma$ é o coeficiente de atrito viscoso e $\vec{F}_{a}(t)$ é uma força aleatória que representa o bombardeamento incessante das moléculas do fluido, (Tomé e Oliveira, 2001; Salinas, 2008). Considerando que o sistema seja livre de forças externas, temos que a equação de movimento é descrita por uma força de atrito viscoso, $\gamma v$ (dissipativa), e uma força imposta pela colisão das partículas, $F_{a}(t)$. 
Depois de todo um tratamento estatístico, (ver por exemplo (Tomé e Oliveira, 2001)) podemos obter a relação de Stokes-Einstein

$$
D=\frac{k_{B} T}{6 \pi \eta R}
$$

onde para partículas esférica temos

$$
\gamma=6 \pi \eta R
$$

Como propomos no modelo discutido no Capítulo 6, sugerimos que na região de transição o agregado lipídico apresente deformações isotrópicas com grandes flutuações, e também sugerimos que nesta região o DMPG apresente grande permeabilidade ao solvente. Deste modo o coeficiente de atrito viscoso não será dado pela relação de Stokes. E torna-se muito difícil avaliar o tamanho efetivo do objeto.

No trabalho realizado por (Yu e Kaloni, 1988) foi observado que esferas com buracos apresentam $\gamma$ menor, e portanto maior difusão em relação a esferas duras. Mesmo que nossa hipótese para a região de transição do DMPG não esteja fundamentada apenas na formação de poros, sugerimos que o coeficiente $\gamma$, nesta região, também seja menor que o obtido pela relação de Stokes. Deste modo, quando utilizamos a equação de StokesEinstein para obter um diâmetro efetivo, podemos estar subestimando o tamanho da partícula.

Então, quando olhamos para a região de transição do DMPG, supomos estar observando valores de diâmetro efetivos menores que os reais. Deste modo, a dispersão não extrusada será muito mais influenciada pela média $z_{a v}$, que a dispersão extrusada (menos polidispersa). Pois, quando as poucas partículas grandes do DMPG são vistas como partículas supostamente menores, a média da medida deixa de ser tão influenciada pelos tamanhos grandes. Por isso observamos uma diminuição de tamanhos com relação as fases gel e fluida.

No DMPG extrusado supomos que a dispersão lipídica tenha uma variedade de tamanhos muito menor que a observada na dispersão não extrusada. Por isso a medida DLS na região de transição do DMPG pode ser menos influenciada pela média $z_{a v}$. A análise por DLS é mais confiável no estudo de dispersões com índice de polidispersão menor. Mesmo observando um aumento no diâmetro efetivo da região de transição do DMPG extrusado, sugerimos que esse valor ainda está subestimado quando avaliado por DLS, pois o coeficiente de atrito viscoso, $\gamma$ é supostamente menor que o obtido para esferas na relação de Stokes. 


\section{Referências Bibliográficas}

Alakoskela, J., e Kinnunnen, M. (2007). Langmuir, 23, 4203-4213.

Alberts, B., Bray, D., Johnson, A., Lewis, J., Raff, M., Roberts, K., e Walter, P. (2000). Fundamentos da biologia celular: uma introdução à biologia molecular da célula. Artmed.

Barroso, R., Riske, K., Henriques, V., e Lamy, M. (2010). Langmuir, 26(17), 1380513814.

Bergntröm, M., e Pedersen, J. (1999). Physical Chemisty Chemical Physics, 1, 4437-4446.

Berne, R., B.J. anda Pecora. (2000). Dynamic light scattering. Dover.

Brown, W. (1993). Dynamic light scattering, the methods and some aplications. Carendon Press.

Disalvo, E. (1991). Chemistry and Physics of Lipids, 59, 199-206.

Fenkel, J. (2005). Prnicípios de eletrodinâmica clássica. edusp.

Fernandez, R., Riske, K., Amaral, L., e Lamy, M. (2008). Biochim. Biophys. Acta.

Goldman, C. (2001). Phys. Review A, 43, 4500-4509.

Goldman, C., Riske, K., e Lamy-Freund, M. (1999). Phys. Rev. E, 60, 7349-7353.

Heimburg, T. (2007). Thermal biophysics of membranes. Wiley-VHC.

Heimburg, T., e Biltonen, R. (1994). Biochemistry, 33, 9477-9488.

Hiemenz, P. (1984). Polymer chemistry. Marcel Dekker.

Jakes, J. (1995). Collect Czechos Chem. Communic, 60, 1781.

Janiak, M., D.M., S., e Shipley, G. (1979). J. Biol. Chem., 254, 6068-6078.

Johnson, C., e Gabriel, D. (1994). Laser light scattering. Dover. 
Koppel, D. (1972). J. Chem. Phys., 57, 4814.

Lamy-Freund, M., e Riske, K. (2003). Chem. Phys. Lipids, 122, 19-32.

Marsh, D., e Phil, M. (1990). Crc handbook of lipid bilayers. CRC Press.

Nagle, J., e Tristram-Nagle, S. (2000). Biochimica et Biophysica Acta, 1469, 159-195.

Nussenzveig, M. (2008). Curso de fśica básica - 4 óptica, relatividade e física quântica. Edgard Blücher.

Oliveira, M. (2005). Temodinâmica. Livraria da Física.

Riske, K., Amaral, L., Döbereiner, H.-G., e Lamy, M. (2004). Biophys. J., 86, 3722-3733.

Riske, K., Amaral, L., e Lamy, M. (2009). Langmuir, 25(17), 10083-10091.

Riske, K., Amaral, L., e Lamy-Freund, M. (2001). Biochim. Biophys. Acta, 1511, 297-308.

Riske, K., Fernandez, R., Nascimento, O., Bales, B., e Lamy-Freund, M. (2003). Chemistry and Physics of Lipids, 124, 69-80.

Riske, K., Nascimento, O., Peric, M., Bales, B., e Lamy-Freund, M. (1999). Biochim. Biophys. Acta, 1418, 133-146.

Riske, K., Politi, M., Reed, W., e Lamy-Freund, M. (1997). Chem. Phys. Lipids, 89, $31-44$.

Rodembusch, F. (2001). Espalhamento de luz estático e dinâmico em polímeros do tipo polimetilmetacrilato fluorescentes por transferência protônica intramolecular no estado eletrônico excitado (tpiee). Disertção de mestrado.

Sakurai, J. (1994). Modern quantum mechanics. Addison-Wesley Publishing Company.

Salinas, S. (2008). Introdução à física estatística. edusp.

Salonen, I., Eklund, K., Virtanen, J., e Kinnunen, P. (1989). Biochim. Biophys. Acta, 982, 205-215.

Smith, W., e Henderson, D. (1970). Molecular Physics, 19, 411-415.

Spinozzi, F., Paccamiccio, L., Mariani, P., e Amaral, L. Q. (2010). Lagmuir, 26(9), 6486-6493.

Tamashiro, M., Henriques, V., e Lamy, M. (2005). Langmuir, 21, 11005-11016. 
Tomé, T., e Oliveira, M. (2001). Dinâmica estocástica e irreversibilidade. edusp.

Williams, C. (1962). Methods of experimental physics, vol.3 - molecular physics. Academic Press.

Yi, P., e Mc Donald, R. (1973). Chemistry and Physics of Lipids, 11, 114-134.

Yu, B., e Kaloni, P. (1988). Journal of Appliied Mathematics and Physics, 39, 937-941. 